5urface charge characterieation of gibbsite nanoparticles

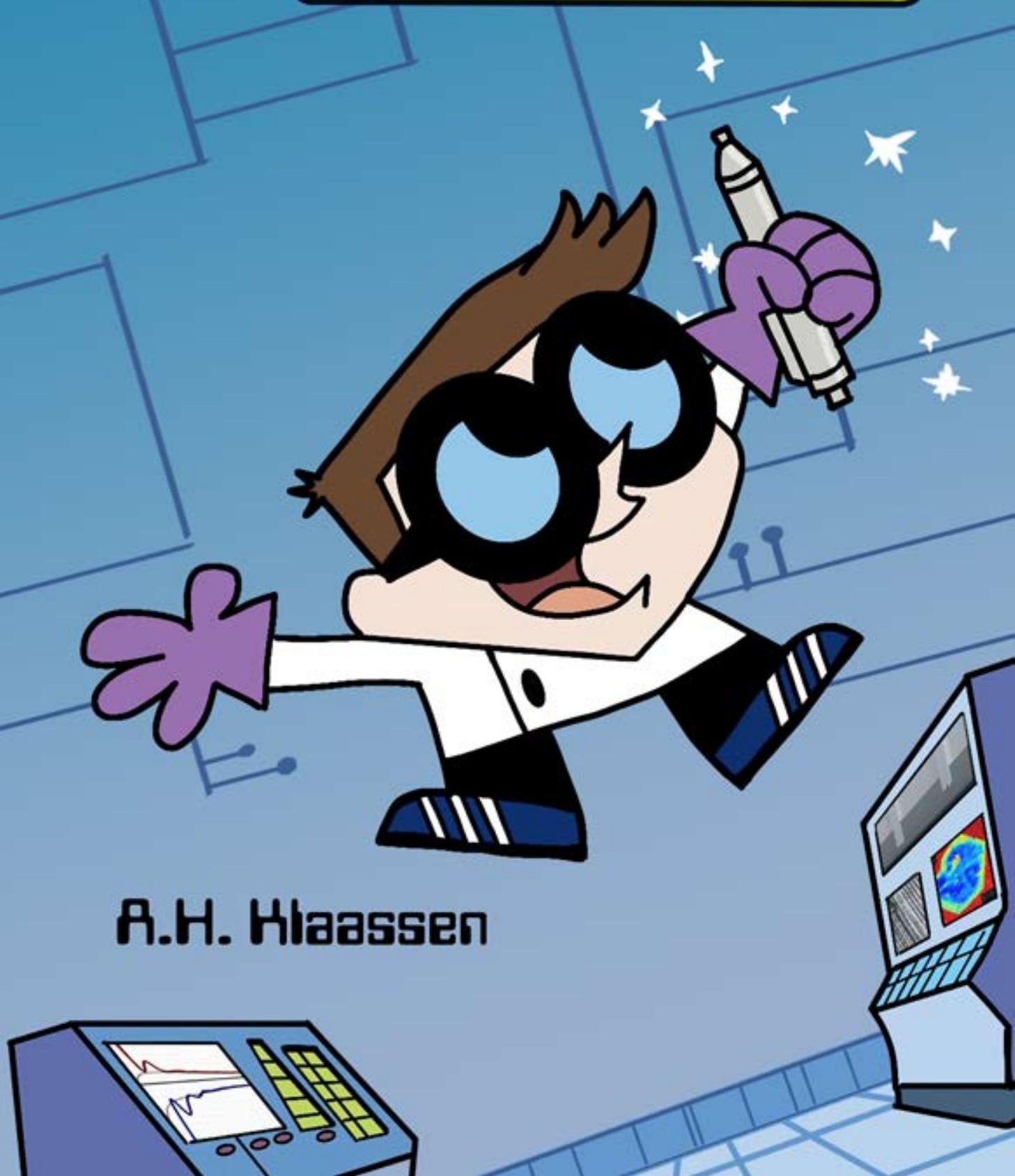




\section{Surface charge characterization of gibbsite nanoparticles}

An atomic force microscope study

Aram H. Klaassen 


\section{Graduation committee:}

Prof. dr. J.L. Herek

Prof.dr. Frieder Mugele

Dr. Igor Siretanu
University of Twente, Chairperson

University of Twente, Promotor

University of Twente, Assistant promoter

\section{Members:}

Prof. dr. Andre ten Elshof

Dr. Remco Hartkamp

Prof. dr. Leon Lefferts

Dr. Kislon Voitchovsky

Prof. dr. Regine von Klitzing
University of Twente

Delft University of Technology

University of Twente

Durham University

Technische Universität Darmstadt

\section{MESA+ \\ INSTITUTE FOR NANOTECHNOLOGY}

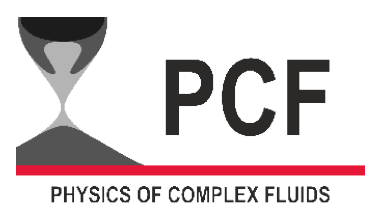

The research described in this thesis was performed at the Physics of Complex Fluids group within the MESA+ Institute for Nanotechnology and the Department of Science and Technology of the University of Twente. This work is part of the ExploRe research program which is financially supported by BP plc.

Title: Surface charge characterization of gibbsite nanoparticles

Author: Aram H. Klaassen

ISBN: $\quad 978-90-365-5071-0$

DOI: $\quad 10.3990 / 1.9789036550710$

Copyright $(\subseteq$ by Aram Klaassen, Borne, the Netherlands.

All rights reserved. No part of this work may be reproduced by print, photocopy or any other means without prior permission in writing of the author.

Cover design by Denise te Paste \& Aram Klaassen.

Printed by Gildeprint, Enschede. 


\title{
SURFACE CHARGE CHARACTERIZATION OF GIBBSITE NANOPARTICLES
}

\author{
DISSERTATION \\ to obtain \\ the degree of doctor at the University of Twente, \\ on the authority of the rector magnificus, \\ prof. dr. T.T.M. Palstra, \\ on account of the decision of the Doctorate Board, \\ to be publicly defended \\ on Wednesday the 11 th of November 2020 at $16: 45$ \\ by \\ Aram Harold Klaassen \\ born on the 9th of February 1988 \\ in Silvolde
}


This dissertation has been approved by:

Promoter:

Assistant promoter:
Prof. dr. Frieder Mugele

Dr. Igor Siretanu 


\section{Contents}

1 Introduction 1

$1.1 \quad$ Enhanced oil recovery $\ldots \ldots \ldots$. . . . . . . . . . 1

1.2 DLVO theory $\ldots \ldots \ldots \ldots \ldots$

1.3 Atomic Force Microscopy . . . . . . . . . . . . . . . . . 9

1.4 Aim of this thesis . . . . . . . . . . . . . 12

2 Materials and methods $\quad 21$

2.1 Introduction to reservoir rocks . . . . . . . . . . . 22

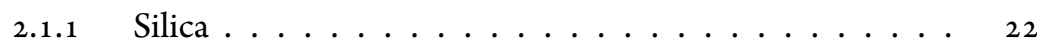

$2.1 .2 \quad$ Clays . . . . . . . . . . . . . . . . 22

2.1 .3 Gibbsite ..................... 23

2.2 Atomic force microscopy . . . . . . . . . . . . . 25

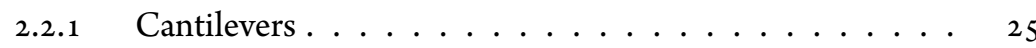

$2.2 .2 \quad$ Static force spectroscopy . . . . . . . . . . . 26

2.2 .3 Dynamic force spectroscopy . . . . . . . . . . 27

2.2 .4 Force inversion ................. 30

2.3 Physical forces experienced by AFM tip . . . . . . . . . . 32

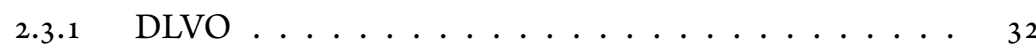

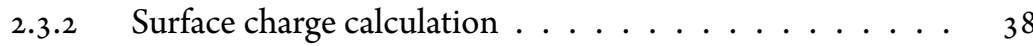

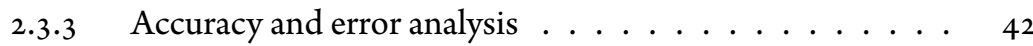

2.4 Principal component analysis . . . . . . . . . . . . . . 43

2.4 .1 PCA applied to AFM data . . . . . . . . . . 46

3 Ionic strength and $\mathrm{pH}$ dependence $\quad \mathbf{5 5}$

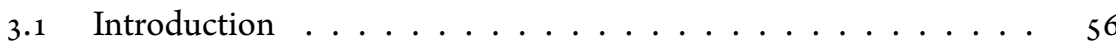

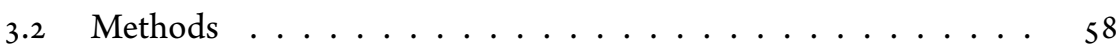


3.2.1 Substrate and probe preparation . . . . . . . . . . 58

3.2 .2 AFM force spectroscopy . . . . . . . . . . . . . . 59

3.2 .3 Data handling .................. 60

3.2.4 Determination of the tip-sample distance . . . . . . . 62

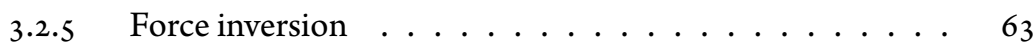

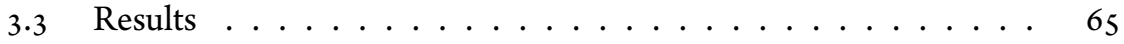

3.4 Discussion . . . . . . . . . . . . . . . . 69

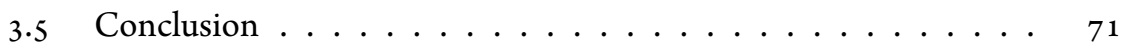

4 Impact of surface defects

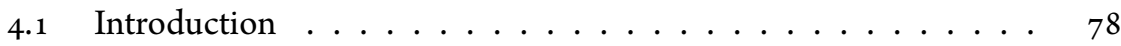

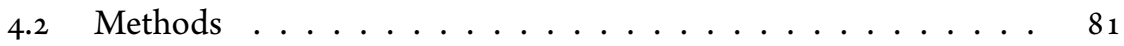

4.3 Results ....................... 88

$4 \cdot 3.1 \quad$ AM-AFM imaging of gibbsite nanoparticles . . . . 88

$4 \cdot 3.2 \quad 3 \mathrm{D}$ force field measurements . . . . . . . . . 88

4.3 .3 Surface charge maps . . . . . . . . . . . . . . . 90

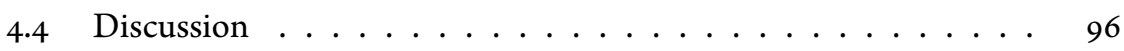

4.5 Conclusion ............................ 101

5 Hydration forces 109

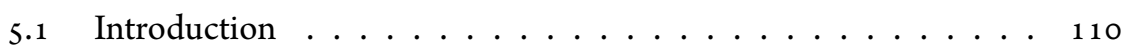

5.2 Methods ..................... 112

5.2 .1 Hydration Forces . . . . . . . . . . . . . . 114

5.3 Results ........................ 115

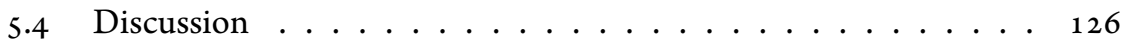

5.5 Conclusion . . . . . . . . . . . . . . . . . 129

5.6 Appendix ...................... 130

$\begin{array}{llr}6 & \text { Divalent ions } & 143\end{array}$

6.1 Introduction .................... 143

6.2 Methods and Materials . . . . . . . . . . . . . . . . 145

$6.2 .1 \quad$ Sample and probe preparation . . . . . . . . . . . 145

6.2.2 Amplitude modulation force spectroscopy . . . . . . . . 145

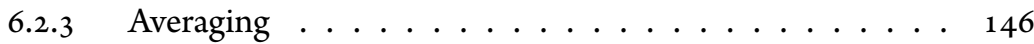

6.2 .4 Cantilevers . . . . . . . . . . . . . . . . 147 
6.3 Results and discussion . . . . . . . . . . . . . . . 148

6.4 Conclusion ...................... 157

\begin{tabular}{lll}
\hline Conclusions and outlook & 161
\end{tabular}

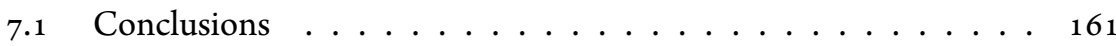

7.2 Outlook. . . . . . . . . . . . . . . 163

$7.2 .1 \quad$ Diffuse layer charge vs intrinsic surface charge . . . . . 163

7.2 .2 Reservoir conditions . . . . . . . . . . . . . 164

$7.2 .3 \quad$ Hydration forces . . . . . . . . . . . . . 167

$\begin{array}{lr}\text { Summary } & 171\end{array}$

$\begin{array}{lr}\text { Samenvatting } & 175\end{array}$

\begin{tabular}{lr}
\hline List of publications & 179
\end{tabular}

\begin{tabular}{lr}
\hline Acknowledgments & 181
\end{tabular}

\begin{tabular}{ll}
\hline About the author & 185
\end{tabular} 



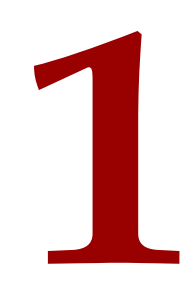

Introduction

IN THIS CHAPTER I will discuss the context of the research presented in this thesis and give an introduction to the solid liquid interface. I give a short introduction of the experimental tools used to explore the solid liquid interface. Furthermore, the lay-out of this thesis is presented.

\subsection{Enhanced oil recovery}

The world still largely depends on oil. Oil fuels our cars, it is used in plastics, the production of nylon, polyester and artificial fur, furniture and it is used in the insulation of our homes. For some of these applications, the use of oil can be replaced by a more environmentally friendly alternative. However, we will not soon be able to completely eradicate the use of oil. Therefore, the search for more oil is still a relevant topic today. The discovery of new oil fields, however, is becoming increasingly 
difficult and therefore the need for improving the recovery factor of existing oil production fields is growing. Globally, the recovery factor, the fraction of oil recovered from the oil reservoir, is only between 20 to $60 \%$ [1] . This means that a large amount of oil remains in the reservoir.

Oil (hydrocarbons) and gas originate from source rocks, which are geological deposits containing organic material. Over a time span of millions of years the oil has been formed from organic matter by bacteria under extreme pressure and temperatures. Formed oil slowly migrates via the pore space between the rock/sand grains to the rock reservoir. The reservoir rock is a layer of porous and permeable rock where the oil is contained. Large parts of the pore space is filled with oil, while a minor fraction $(\approx 20 \%$ ) consists of formation water (water that was trapped during the rock deposition). Typically, reservoirs are a few kilometers long and about a hundred meter thick and are located at a depth of a few kilometers usually. At these depths the pressure and temperature can increase up to $100 \mathrm{MPa}$ and $200{ }^{\circ} \mathrm{C}$, respectively. There are different types of rock reservoir, such as sandstone and carbonates. The term sand refers to the specific grain size (between $62 \mu \mathrm{m}$ and $2 \mathrm{~mm}$. In this thesis I limit myself to sandstone reservoirs.

These conditions hamper oil recovery. Due to the complex nature of the recovery process, a number of extraction techniques are deployed, which can be categorized by the phase at which they are applied during the recovery process.

The primary oil recovery is the first phase, which happens once a well is drilled from the surface to the underground reserve (also called a well-bore). The recovery continues until the pressure inside the well is no longer enough to produce a profitable quantity of oil. The pressure in the well can originate from gas drive, groundwater drive, or gravity drainage. This phase only produces about $10 \%$ of a reserves supply.

The secondary phase consists of creating a pressure gradient in the reservoir by injecting another medium (gas, water) through injection wells (Figure 1.1 a). The injected fluid is supposed to displace the oil and drive it to the production well-bore. This usually results in a recovery of up to another 30-50\%. After the primary and secondary recovery, about $30-60 \%$ of the oil is still trapped in the reservoir. The exact amount varies between oil reservoirs, since the recovery process is determined by the properties of the oil and the characteristics of the reservoir rock. Properties such as the 

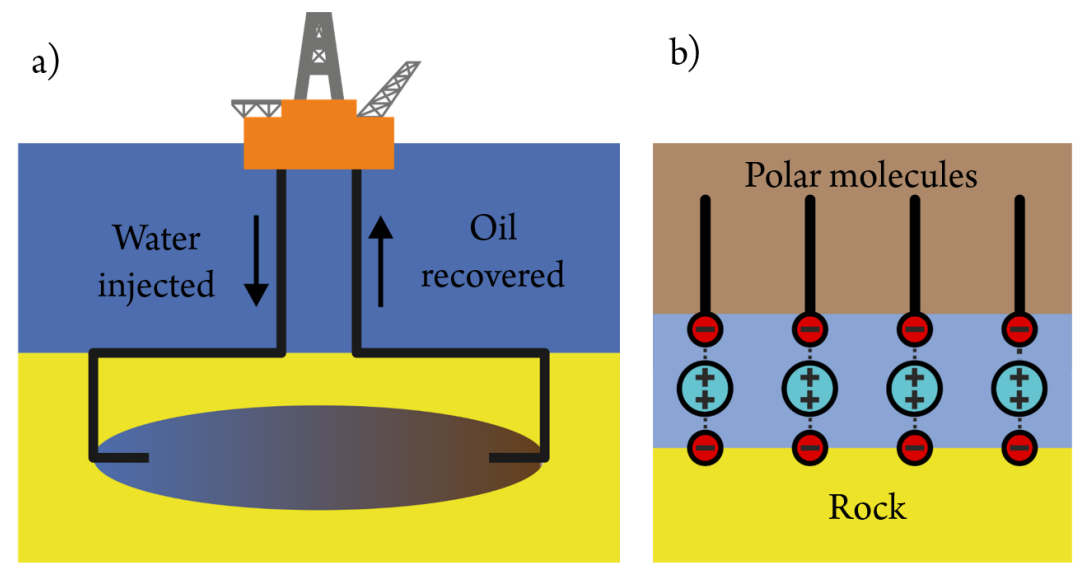

Figure 1.1: a) Cartoon of the secondary phase of oil recovery. By applying a pressure gradient over the reservoir the oil is supposed to be displaced. b) Divalent ions form a bridge between the negatively charged rock surface and negatively charged molecules in the oil.

spatial distribution of pores [2] ${ }^{[2]}$, wettability ${ }^{[3]}$, heterogeneity in rock permeability $[$ [1] and clay content of the reservoir [4] influence the recovered amount of oil.

If the oil price is high enough and if it is financially feasible, tertiary recovery methods, also known as enhanced oil recovery (EOR), are used [5,6]. EOR methods include thermal recovery, gas injection, chemical injection [7] and low-salinity water flooding $[8,1,9,10]$. Low salinity water flooding (LSWF) is typically used on offshore platforms, where the salt content of the seawater is reduced before the injection $[8]$. By using LSWF an additional 5-20\% of oil can be extracted from the reservoir.

Low salinity water flooding Pressure and temperature are not the only factors that complicate the oil recovery process. Organic components in the crude oil, rock, and brine (sea water) have a large influence on the effectiveness of the deployed extraction techniques.

The crude oil in the oil reservoirs is not composed of a single substance, but it is a mixture of many different organics [11] . The main components are hydrocarbons, in the form of alkanes, cycloalkanes, aromatic hydrocarbons, water soluble organics, and polar organic compounds. Also, saline water is a mixture containing a wide variety 
of salts, that originate from the formation water and injection water. Furthermore, the sandstone is not built from a single material either. It is a porous, fragmented, sedimentary rock composed of minerals and rock grains. Its composition is mainly quartz and feldspar with cementing materials like silica, calcium carbonate, montmorillonite and kaolinite. Additionally, the composition of each oil reservoir is different, which has resulted in the development of a considerable number of techniques for oil recovery.

All these different ingredients result in a complex system in which all of its components are able to interact with each other. This makes identifying the key mechanism behind LSWF challenging. Early work in the 1990's studied the effect of brine composition during core floods of sandstone cores $\left[{ }_{12}-14\right]$. The injection of low salinity brine in cores containing clays and crude oil was shown to increase the recovery by $15-40 \%$. From field experiments, laboratory core floods and inter-well tests it is believed that the primary trigger for the enhanced oil recovery through LSWF is the macroscopic wettability alteration of the rock reservoir $[10,15-18,3,19-21]$. The change in affinity of the rock reservoir from an oil preferred wetting to a water preferred wetting, forces the oil to be replaced by water resulting in an increased oil recovery.

A number of requirements for the low salinity have been identified, which are: a) the presence of clays (like kaolinite, illite and montmorillonite) or some type of negatively charged rock surfaces. b) Polar components in the oil phase. c) Presence of divalent ions or multicomponent ions in the formation water. Therefore, quantifying the surface charge of clays and rock surfaces as function of $\mathrm{pH}$ and monovalent and divalent ions can help the understanding of the chemical processes involved. [11].

After a few decades of research, the surface charge origin of clay particles in general is still not well understood. This does not only hold for the surface charge, but also for how ions adsorb onto the surface and how clays are hydrated. Clays like kaolinite and illite are composed from aluminum hydroxide (gibbsite) and silicate sheets. For gibbsite it is believed that the edges have a $\mathrm{pH}$ dependent charge. Whether the basal planes are charged is unclear. Also, the origin of the charge is unclear $[22,23]$. On kaolinite, the basal plane is generally believed to come from isomorphous substitution, where ions in the crystal lattice are replaced by ions of a different valency [24,25]. 
This suggests that the basal plane is mainly $\mathrm{pH}$ independent. However, from measurements a clear $\mathrm{pH}$ dependent charge was observed [26,27]. This $\mathrm{pH}$ dependency is suspected to arise from the (de)protonation of exposed surface groups at the edge of the clay particle. Also, is the $\mathrm{pH}$ dependency influenced by surface defects, since in the chemical modeling this is often not taken into account [28- -31$]$. Moreover, how the hydration of clays affects ion adsorption and the adsorption/desorption processes is not well understood either [17].

\subsection{DLVO theory}

The solid liquid interface is a complex environment where several forces are at play. We start to describe those with the Derjaguin [32], Landau, Verwey [33] and Overbeek (DLVO) theory. The DLVO theory describes how the pressure between two surfaces is the sum of the van der Waals force and the electrostatic force.

Van der Waals A long range force that is present in all environments is the van der Waals force, an intermolecular interaction force that acts between atoms and molecules due to their charge and electronic distribution. This force is in most environments attractive. However, not all particles stick together, so there should also be an opposing force.

Double layer The repulsive force that can prevent particles from sticking together (in solutions), is the electrostatic force. Electrostatic forces result from the interaction of charged surfaces. A surface in contact with water or another organic solvent can acquire a charge through different mechanisms. One mechanism is the ionization or dissociation of surface groups. The surface is, for example, terminated by - $\mathrm{OH}$ groups. When a proton $\left(\mathrm{H}^{+}\right)$dissociates (deprotonation), it leaves a negatively charged surface group behind $\left(-\mathrm{O}^{-}\right)$. Another mechanism is the adsorption or binding of ions onto the surface. In the previous example, a positively charged ion, such as $\mathrm{Na}^{+}$, can neutralize the negative charge of the surface group. Note that ions can also chemically adsorb to a surface. In this case, charge does not play the dominant role in the adsorption process.

In the bulk solution, far away from any charged surface, the co-ions and counter-ions are equally distributed. Here, the solution is charge neutral. Near the surface, the 


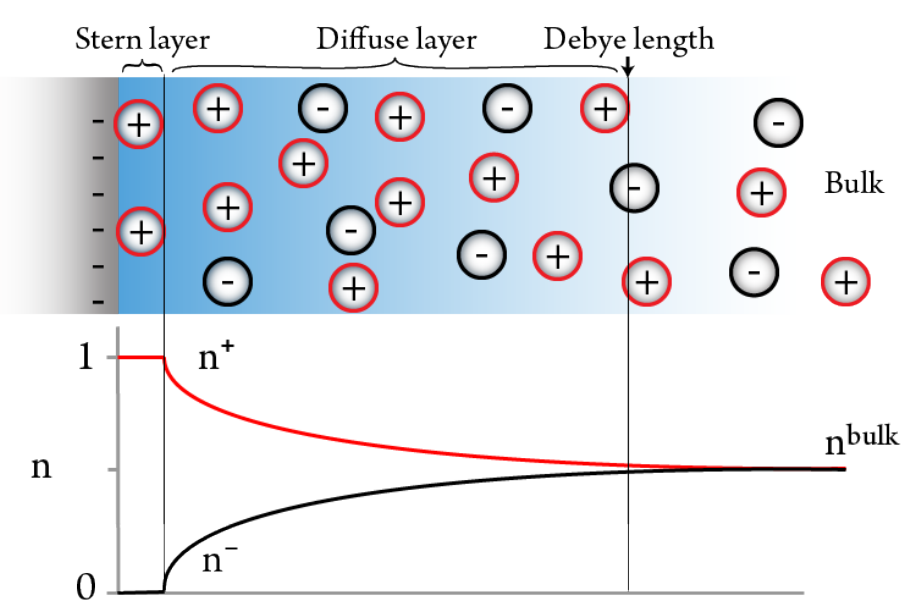

Figure 1.2: On top a schematic representation of an electric double layer in contact with a negatively charged surface with a fixed layer of positively charged ions adsorbed on the surface. On the bottom a graph indicating the number density of positive and negative ions (red and black line). The length of the Debye length $\kappa_{D}$ is where the magnitude of the electrical potential decreases to $1 / e$. 
equal distribution of ions is disturbed by the surface charge $\left(\sigma_{\circ}\right)$ or surface potential. Ions with a counter charge are attracted, while ions with the same charge are repelled. This leads to a reordering of the ions in the region near the surface. This region is called the diffuse layer and is schematically shown in figure 1.2, having a charge $\sigma_{D}$. Ions can also adsorb to the surface, forming a layer, which is called the Stern Layer and has a charge $\sigma_{D}$. The Stern layer and the diffuse layer together, form the electric double layer. The (intrinsic) surface charge, Stern layer charge and diffuse layer charge are together charge neutral $\left(\sigma_{\mathrm{o}}+\sigma_{S}=-\sigma_{D}\right)$.

The concentration of the counter- and co-ions in the diffuse layer decays as a function of distance from the surface. The potential of the surface has its maximum value at the surface and is zero in the bulk. The concentration profile that follows has an exponential decay described by the Gouy-Chapman model. An exponential decay has a decay rate which in this model is called the Debye parameter $\kappa$. The Debye length $\left(\kappa^{-1}\right)$ is the characteristic length of the diffuse layer, which is where the magnitude of the electrical potential has dropped to $1 / \mathrm{e}$. The thickness of this layer is determined by the concentration of the ions present in the solution.

The diffuse layer charge and the charge distribution in the electric double layer are influenced by the properties of the solution, like $\mathrm{pH}$ and electrolyte. The $\mathrm{pH}$ (concentration $\mathrm{OH}^{-}$or $\mathrm{H}^{+}$) can change the equilibrium of the deprotonation reaction $\left(\mathrm{S}-\mathrm{OH} \rightleftharpoons \mathrm{S}-\mathrm{O}^{-}+\mathrm{H}^{+}\right)$. When the $\mathrm{pH}$ is high (high $\mathrm{OH}^{-}$concentration), the balance of the reaction formula is shifted to the right, leading to more deprotonation (more $\mathrm{S}-\mathrm{O}^{-}$groups). More deprotonation leads to a more negatively charged surface. In order to investigate the influence of the fluid compositions on the surface charge, the experiments in this thesis are often performed with solutions of various $\mathrm{pH}$ and electrolyte type and concentration.

The forces that arise when two surfaces approach closely, i.e. their electric double layers start to overlap, can be calculated using the potential profile of the electric double layer. The potential profile is the description of how the potential changes as function of the distance from the surface. Poisson-Boltzmann theory is used to describe the relation of the potential profile and charge density. This theory and how it is used to extract diffuse layer charge values will be described in chapter 2 .

Beyond DLVO The DLVO theory is a continuum theory and therefore does not describe the discrete nature of the solution (water molecules and ions). The con- 


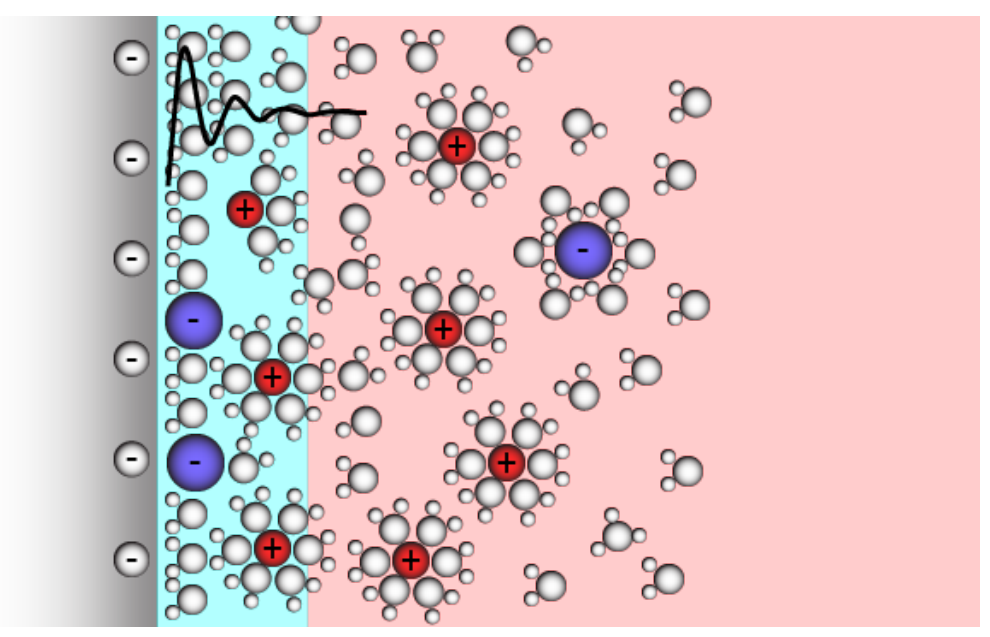

Figure 1.3: A schematic representation of an electric double layer with hydrated ions and water molecules. At the top a representation of water layering at the surface with a decaying oscillatory function.

sequence of this non-discrete nature of the theory is that it breaks down at small distances from the surface $(\approx<2 \mathrm{~nm})$. At these distances the size and interactions of these particles involved start to have a significant effect on measured interaction forces, causing the measured forces and the theory to deviate. The DLVO theory has been improved over time to include a variety of sometimes competing microscopic effects. For example, ion correlation effects [34] that take into account the finite size of the ion and its polarizability. Or the preferential binding of ions to specific surface sites $[22,29]$ and non-electrostatic potentials acting between ions and colloidal surfaces [35]. Despite all proposed improvements, it will unlikely be able to describe the complex structure of the solid liquid interface in the cartoon in figure 1.3 .

In figure 1.3 we can see the layer of water molecules at the interface. In this example there is only one layer, however, there can be many more layers. The organizing of those water molecules in layers generally weakens further away from the surface. The black line in figure 1.3 shows an example of how the density profile of the water molecules can look like. It has a periodicity of the size of the molecules and the amplitude decays over distance. When another surface approaches, both density dis- 
tributions start to overlap resulting in an alternating increase and decrease of the total density. The force to overcome those densities can be measured as an oscillatory hydration (solvation when it is not about water) force and only arises because of the disruption of the organized layers by a second surface (the black graph is actually a measured hydration force from chapter 5 ). The first solvation forces were measured using a surface force apparatus (SFA) using microscopic mica sheets in 1980 [36, 37] This was the first evidence of interfacial liquids not behaving bulk-like. Later, the first atomic force microscope (AFM) measurements on such oscillatory forces were reported [38]. AFM allowed for detecting hydration forces on samples that were not macroscopic $\left(\approx_{1} \mathrm{~cm}\right.$, and enabled the study of surface specificity of the solvation forces as well. In figure 1.3 ions are shown as well. How these ions affect the hydration force or the hydration of the ions themselves is actually not well understood [39]. Moreover, how the surface charge depends on the surface chemistry and charge is not well understood either. To add to this, the oscillatory force as shown in figure 1.3 is often superimposed on a monotonic hydration force. This force remains an open topic in literature [40,41].

\subsection{Atomic Force Microscopy}

As shortly mentioned above, the AFM can be used to do a multitude of surface characterizations. Here I explain the basics of atomic force microscopy. The Atomic Force Microscope was invented in 1986 by Binnig, Quate and Geber [42] , shortly after the scanning tunneling microscope. The main feature of all scanning probe microscopes is that they map the topography (or another surface property) by scanning a 'sharp' probe over a surface. A schematic (dimensions not to scale) is shown in figure 1.4, where the basic functions of the AFM are shown. In chapter 2 a more detailed description of AFM techniques is presented. Here, we focus on the basic principles of AFM. The AFM uses a tip that is mounted on the edge of a flexible cantilever. When a sample is scanned, the interaction forces between the tip and surface push the cantilever out of equilibrium, causing the cantilever to deflect. This deflection can be monitored with the four-quadrant photo-detector through the changing angle of reflection of the red detection laser. The photo-detector registers a voltage and which can later be converted into a force. The sample, in figure $1.4 \mathrm{a}$ gibbsite particle on silica, is glued on a magnetic puck, which magnetically sticks on the sample 


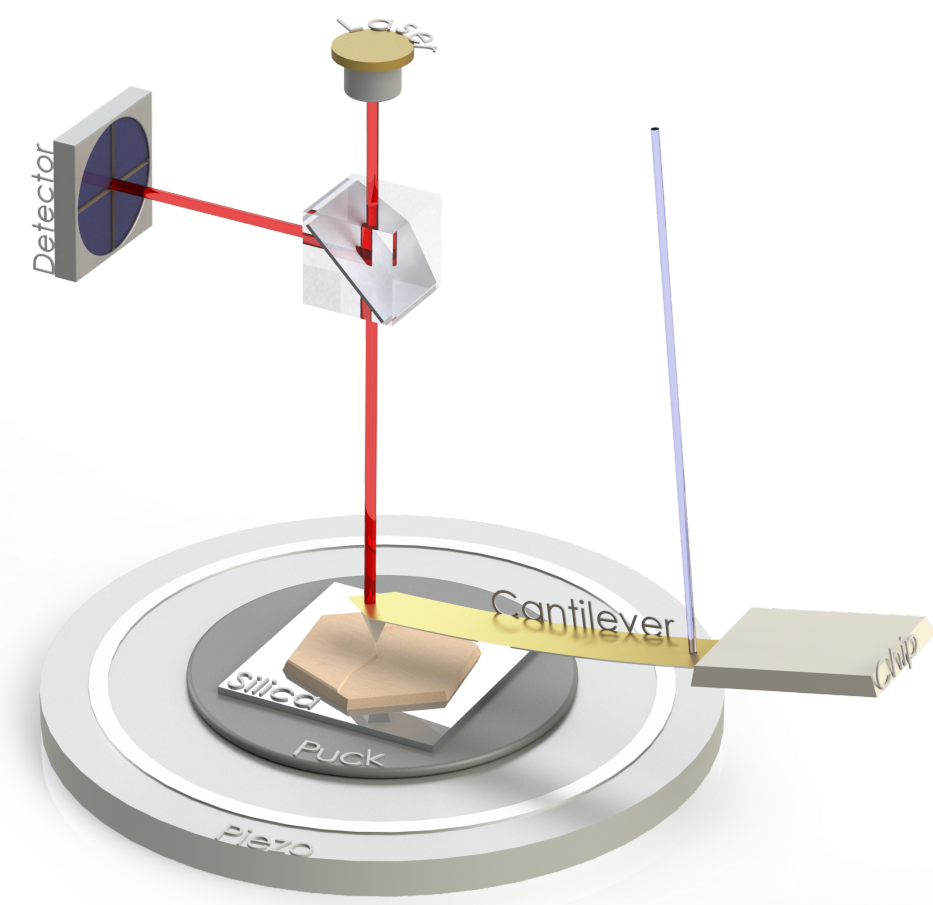

Figure 1.4: A schematic setup of an Atomic Force Microscope. A gibbsite particle sits on top of a square silica substrate which is glued onto a magnetic puck which is placed on the piezo. A cantilever with a tip is used to interact with the sample, while with the red laser the deflection of the cantilever is monitored with the quad photodetector. The blue laser can be used to drive the cantilever. 
stage. When scanning the sample, the cantilever remains in the same location, while the piezo is used to actually move the sample-stage and sample around. The piezoelectric scanner is a positioning device which is capable of moving the sample in $x$-, $y$ - and $z$-direction with Angstrom accuracy. By moving the sample instead of the detection laser (and potentially the blue laser used for photothermal excitation), the optics do not have to be moved during the scans. The cantilever can also be driven, meaning that the cantilever oscillates with a certain amplitude and frequency. The forces that are measured using this method are inferred from the detuning of the oscillator. Driving the cantilever has some advantages. Softer samples can be imaged and information about dissipation can be retrieved. Conventionally, a piezoelectric actuator is used to drive the cantilever, but this may lead to unwanted oscillations in the system which can disturb the measurement. Instead of using a piezoelectric actuator, a laser with periodically varying intensity can be used to oscillate the cantilever. In figure 1.4, a blue laser is aimed at the base of the cantilever. Due to the bimetallic effect (the silicon cantilever needs a coating of a different material on top, like gold) the cantilever starts to oscillate. Using photothermal excitation, the unwanted oscillations caused by piezoelectric actuation can be eliminated. This results in an amplitude frequency response that almost perfectly reflects simple harmonic oscillator theory. Despite that, the quantitative interpretation of the interaction force is different for photothermal excitation from the piezoelectric actuation, which is not often taken into account.

The AFM is a versatile tool which can be used to do a wide variety of things. It can be used to visualize the water distribution at the solid-liquid interface $[43,44]$, show adsorbed ions in the Stern layer ${ }^{[45]}$, measure the diffuse layer charge heterogeneity of mineral surfaces $[46]$, reveal the atomic structure of surfaces and visualize the conformity of DNA molecules [47]. Moreover, it does not suffer from the averaging character of techniques like potentiometric titration, zeta-potential measurements and electrokinetic phoresis, which do offer a good statistical average over many particles. AFM however, can reveal more detailed information about the microscopic aspects of the chemical processes of the solid liquid interface with resolution at nanometer scale, parallel and perpendicular to surfaces $[48-51,45]$. That is why AFM will be used in this thesis to characterize the solid liquid interface. 


\subsection{Aim of this thesis}

Questions that were posted in the sections above reveal that our understanding of the solid liquid interface is still incomplete. The chemical modeling of relatively simple surfaces fails when incorporating for a wide range of salt concentrations and $\mathrm{pH}$ values. This is further hampered by the lack of systematic studies that probe a wide range of electrolyte solutions. But also questions like how water molecules form self organized layers at the interface, how these layers depend on the surface crystallinity, whether they are surface charge dependent and the origin of the monotonic hydration are still largely unanswered. In this thesis, I will address some of those questions, in the following order.

In chapter 2 we will try to further understand the implications of photothermal excitation on the measured forces in Atomic Force Microscopy. At this point often the same force inversion routine is used for acoustic and photothermal excitation, which does not necessarily give equal results. The force inversion routine also depends on the tip-sample geometry, which will be given for commonly used tip-sample geometries. Furthermore, the procedure to extract diffuse layer charge values from the forces will be described, using charge regulation boundary conditions. Since the charge regulation boundary conditions require a chemical model, which is not always available, we develop an exponential surface charge model. This model enables the separation of diffuse layer charge/potential calculations and surface chemistry. Finally, we explore the added value of principal component analysis, which in other scientific fields can improve the data quality significantly.

A colloidal probe has a large interaction area and can be used when the primary interest is to measure the interaction force precisely, at costs of the lateral resolution. In chapter 3 we will characterize the diffuse layer charge using colloidal probe force spectroscopy of a typical rock surface, in this case silica. To do so, we accurately determine the diffuse layer charge over a wide range of $\mathrm{pH}$ and sodium chloride concentration, without any specific chemical knowledge of the surface. The diffuse layer charge values serve as an input for the chemical modeling, with the aim to verify whether the surface can be described with chemical equilibrium constants. Usually these constants are determined for smaller ranges of $\mathrm{pH}$ and salt concentration, where possible fitting issues or deviations are less likely to occur. 
As discussed in the introduction, clays can have a significant effect on the amount of released oil. Often, in studies where the influence of these clays is analyzed, the crystallographic planes of these minerals are modeled as smooth surfaces. The surfaces do not have any broken bonds, lattice imperfections or other defects. In other words, the clay surfaces have a homogeneous distributed diffuse layer charge density. In chapter 4 we measure the diffuse layer charge of gibbsite. We do this using sharp tips, with the aim to capture the homogeneity, or heterogeneity of the diffuse layer charge distribution of gibbsite. We show that even the surface of synthesized gibbsite contains many defects, and that these defects are more $\mathrm{pH}$ responsive than the basal plane of gibbsite. Surface defects are often expected to have an impact on clay mineral surfaces, however, its impact on the diffuse layer charge is shown here for the first time.

The affinity of oil towards clay minerals is in a large part determined by interaction forces such as electrostatics and hydration forces. In most studies only one part of these interaction forces is studied, like in chapter 3 and 4 . In chapter 5 we measure the lateral distribution of hydration forces, while simultaneously measuring the electrostatic force on silica and gibbsite. Usually, these are two separate measurements, because of the different length scales involved. Combining both is unprecedented and combining both allows us to bridge the gap between colloidal scale continuum DLVO forces and molecular scale hydration forces.

In previous chapters we have used monovalent salt for our ambient electrolyte solution. However, as mentioned previously, it is the divalent ions that play an important role in retaining oil in the reservoir. In previous work, it was shown that in $\mathrm{pH} 6$ solutions the calcium ions adsorb to the basal plane of gibbsite. DFT calculations were able to reproduce this and to ensure charge neutrality $\mathrm{OH}^{-}$ions were introduced. One of the observations was that these ions were involved in stabilizing the calcium ions on the gibbsite surface. To study the validity of these findings, the diffuse layer charge of gibbsite was measured in various $\mathrm{pH}$ solutions in chapter 6. At high concentrations $(>50 \mathrm{mM})$ the anion adsorbs on top of the calcium ion. To investigate whether this is chemically specific, we also use solutions of calcium with different anions. 


\section{Bibliography}

[1] A. Muggeridge, A. Cockin, K. Webb, H. Frampton, I. Collins, T. Moulds, and P. Salino, "Recovery rates, enhanced oil recovery and technological limits," Philosophical Transactions of the Royal Society a-Mathematical Physical and Engineering Sciences, vol. 372, no. 2006, 2014.

[2] H. Aksulu, D. Hamso, S. Strand, T. Puntervold, and T. Austad, "Evaluation of low-salinity enhanced oil recovery effects in sandstone: Effects of the temperature and ph gradient," Energy \& Fuels, vol. 26, no. 6, pp. 3497-3503, 2012.

[3] J. S. Buckley and Y. Liu, "Some mechanisms of crude oil/brine/solid interactions," Journal of Petroleum Science and Engineering, vol. 20, no. 3-4, pp. 155160, 1998.

[4] W. H. Somerton and C. J. Radke, "Role of clays in the enhanced recovery of petroleum from some california sands," Journal of Petroleum Technology, vol. 35, no. 3 , pp. $643-654,1983$.

[5] F. I. Stalkup, "Status of miscible displacement," Journal of Petroleum Technology, vol. 35 , no. 4 , pp. $815-826,1983$.

[6] Y. Li, "Oil recovery by low salinity water injection into a reservoir: A new study of tertiary oil recovery mechanism," Transport in Porous Media, vol. 90, no. 2, pp. 333-362, 2011.

[7] B. S. Shiran and A. Skauge, "Enhanced oil recovery (eor) by combined low salinity water/polymer flooding," Energy \& Fuels, vol. 27, no. 3, pp. 1223-1235, 2013.

[8] J. S. H. Lo and R. T. Lassau, "Enhanced oil-recovery," Cim Bulletin, vol. 78, no. 878 , pp. 91-91, 1985 .

[9] H. A. Nasr-El-Din and K. C. Taylor, "The role of surfactants in enhanced oil recovery," Micelles, Microemulsions, and Monolayers, pp. 249-287, 1998.

[10] A. Lager, K.J. Webb, C. J.J. Black, M. Singleton, and K. S. Sorbie, "Low salinity oil recovery - an experimental investigation," Petrophysics, vol. 49, no. 1, pp. 28 $35,2008$. 
[11] L. Yue, W. Pu, S. Zhao, S. Zhang, F. Ren, and D. Xu, "Insights into mechanism of low salinity water flooding in sandstone reservoir from interfacial features of oil/brine/rock via intermolecular forces," Journal of Molecular Liquids, vol. 313 , 2020.

[12] P. P. Jadhunandan and N. R. Morrow, "Spontaneous imbibition of water by crude-oil brine rock systems," In Situ, vol. 15, no. 4, pp. 319-345, 1991.

[13] G. Q. Tang and N. R. Morrow, "Influence of brine composition and fines migration on crude oil/brine/rock interactions and oil recovery," Journal of Petroleum Science and Engineering, vol. 24, no. 2-4, pp. 99-111, 1999.

[14] H. O. Yildiz and N. R. Morrow, "Effect of brine composition on recovery of moutray crude oil by waterflooding," Journal of Petroleum Science and Engineering, vol. 14, no. 3-4, pp. 159-168, 1996.

[15] M. D. Jackson, J. Vinogradov, G. Hamon, and M. Chamerois, "Evidence, mechanisms and improved understanding of controlled salinity waterflooding part 1: Sandstones," Fuel, vol. 185, pp. 772-793, 2016.

[16] O. R. Wagner and R. O. Leach, "Improving oil displacement efficiency by wettability adjustment," Transactions of the American Institute of Mining and Metallurgical Engineers, vol. 216, pp. 65-72, 1959.

[17] S. F. Shariatpanahi, S. Strand, and T. Austad, "Evaluation of water-based enhanced oil recovery (eor) by wettability alteration in a low-permeable fractured limestone oil reservoir," Energy \& Fuels, vol. 24, no. 11, pp. 5997-6008, 2010.

[18] M. Kumar, A. Fogden, N. R. Morrow, and J. S. Buckley, "Mechanisms of improved oil recovery from sandstone by low salinity flooding," Petrophysics, vol. 52, no. 6, pp. 428-436, 2011.

[19] P. F. Mugele, I. Sîretanu, N. Kumar, B. Bera, L. Wang, M. Maestro, D. M. Duits, D. H. v. d. Ende, and I. Collins, "Charge control and wettability alteration at solid-liquid interfaces," 2014. 
[20] F. Mugele, B. Bera, A. Cavalli, I. Siretanu, A. Maestro, M. Duits, M. CohenStuart, D. van den Ende, I. Stocker, and I. Collins, "Ion adsorption-induced wetting transition in oil-water-mineral systems," Sci Rep, vol. 5, p. 10519, 2015.

[21] F. Mugele, I. Siretanu, N. Kumar, B. Bera, L. Wang, R. de Ruiter, A. Maestro, M. Duits, D. van den Ende, and I. Collins, "Insights from ion adsorption and contact-angle alteration at mineral surfaces for low-salinity waterflooding," SPE Journal, 2016.

[22] T. Hiemstra, P. Venema, and W. Van Riemsdijk, "Intrinsic proton affinity of reactive surface groups of metal (hydr) oxides: The bond valence principle," Journal of colloid and interface science, vol. 184, no. 2, pp. 680-692, 1996.

[23] X. Liu, J. Cheng, M. Sprik, X. Lu, and R. Wang, "Understanding surface acidity of gibbsite with first principles molecular dynamics simulations," Geochimica et Cosmochimica Acta, vol. 120, pp. 487-495, 2013.

[24] E. Tombacz and M. Szekeres, "Surface charge heterogeneity of kaolinite in aqueous suspension in comparison with montmorillonite," Applied Clay Science, vol. 34, no. 1-4, pp. 105-124, 2006.

[25] J. Lutzenkirchen, A. Abdelmonem, R. Weerasooriya, F. Heberling, V. Metz, and R. Marsac, "Adsorption of dissolved aluminum on sapphire-c and kaolinite: implications for points of zero charge of clay minerals," Geochem Trans, vol. 15, p. 9, 2014.

[26] V. Gupta and J. D. Miller, "Surface force measurements at the basal planes of ordered kaolinite particles," Journal of Colloid and Interface Science, vol. 344, no. 2, pp. 362-371, 2010.

[27] N. Kumar, C. Zhao, A. Klaassen, D. van den Ende, F. Mugele, and I. Siretanu, "Characterization of the surface charge distribution on kaolinite particles using high resolution atomic force microscopy," Geochimica et Cosmochimica Acta, vol. 175, pp. 100-112, 2016.

[28] T. Hiemstra, W. Van Riemsdijk, and G. Bolt, "Multisite proton adsorption modeling at the solid/solution interface of (hydr) oxides: A new approach: I. model description and evaluation of intrinsic reaction constants," Journal of colloid and interface science, vol. 133, no. 1, pp. 91-104, 1989. 
[29] G. Sposito, The Environmental Chemistry of Aluminum, Second Edition. Taylor and Francis, 1995.

[30] S. Goldberg, J. A. Davis, and J. D. Hem, "The surface chemistry of aluminum oxides and hydroxides," The environmental chemistry of aluminum, pp. 271-331, 1996.

[31] R. A. Yokel, "Aluminum," Elements and Their Compounds in the Environment: Occurrence, Analysis and Biological Relevance, Second Edition, pp. 635-658, 2004.

[32] B. Derjaguin and L. Landau, "The theory of stability of highly charged lyophobic sols and coalescence of highly charged particles in electrolyte solutions," Acta Physicochim. URSS, vol. 14, no. 633-662, p. 58, 1941.

[33] E. J. W. Verwey, "Theory of the stability of lyophobic colloids," The Journal of Physical and Colloid Chemistry, vol. 51, no. 3, pp. 631-636, 1947.

[34] D. Ben-Yaakov, D. Andelman, D. Harries, and R. Podgornik, "Beyond standard poisson-boltzmann theory: ion-specific interactions in aqueous solutions," $J$ Phys Condens Matter, vol. 21, no. 42, p. 424106, 2009.

[35] D. F. Parsons and B. W. Ninham, "Importance of accurate dynamic polarizabilities for the ionic dispersion interactions of alkali halides," Langmuir, vol. 26, no. 3, pp. 1816-23, 2010.

[36] J. N. Israelachvili, Intermolecular and surface forces : with applications to colloidal and biological systems. London ; Orlando, Fla .: Academic Press, 1985.

[37] R. G. Horn, D. T. Smith, and W. Haller, "Surface forces and viscosity of water measured between silica sheets," Chemical Physics Letters, vol. 162, no. 4, pp. 404-408, 1989.

[38] S. J. O'Shea and M. E. Welland, "Atomic force microscopy at solid-liquid interfaces," Langmuir, vol. 14, no. 15, pp. 4186-4197, 1998.

[39] R. M. Pashley, "Hydration forces between mica surfaces in aqueous electrolyte solutions," Journal of Colloid and Interface Science, vol. 8o, no. 1, pp. 153-162, 1981. 
[40] V. A. Parsegian and T. Zemb, "Hydration forces: Observations, explanations, expectations, questions," Current Opinion in Colloid \& Interface Science, vol. 16, no. 6, pp. 618-624, 2011.

[41] B. R. Shrestha and X. Banquy, "Hydration forces at solid and fluid biointerfaces," Biointerphases, vol. 11, no. 1, p. $018907,2016$.

[42] G. Binnig, C. F. Quate, and C. Gerber, "Atomic force microscope," Physical Review Letters, vol. 56, no. 9, pp. 930-933, 1986.

[43] T. Fukuma, Y. Ueda, S. Yoshioka, and H. Asakawa, "Atomic-scale distribution of water molecules at the mica-water interface visualized by three-dimensional scanning force microscopy," Physical Review Letters, vol. 104, no. 1, 2010.

[44] K. Kimura, S. Ido, N. Oyabu, K. Kobayashi, Y. Hirata, T. Imai, and H. Yamada, "Visualizing water molecule distribution by atomic force microscopy," $J$ Chem Phys, vol. 132, no. 19, p. 194705, 2010.

[45] I. Siretanu, D. Ebeling, M. P. Andersson, S. L. Stipp, A. Philipse, M. C. Stuart, D. van den Ende, and F. Mugele, "Direct observation of ionic structure at solidliquid interfaces: a deep look into the stern layer," Sci Rep, vol. 4, p. 4956, 2014.

[46] N. Kumar, M. P. Andersson, D. van den Ende, F. Mugele, and I. Siretanu, "Probing the surface charge on the basal planes of kaolinite particles with highresolution atomic force microscopy," Langmuir, vol. 33, no. 50, pp. $14226-$ $14237,2017$.

[47] H. Kominami, K. Kobayashi, and H. Yamada, "Molecular-scale visualization and surface charge density measurement of z-dna in aqueous solution," Sci Rep, vol. 9, no. 1, p. 6851, 2019.

[48] T. Fukuma and R. Garcia, "Atomic- and molecular-resolution mapping of solidliquid interfaces by $3 \mathrm{~d}$ atomic force microscopy," ACS Nano, vol. 12, no. 12, pp. 11785-11797, 2018.

[49] T. Fukuma, B. Reischl, N. Kobayashi, P. Spijker, F. F. Canova, K. Miyazawa, and A. S. Foster, "Mechanism of atomic force microscopy imaging of threedimensional hydration structures at a solid-liquid interface," Physical Review B, vol. 92, no. 15, 2015 . 
[50] I. Siretanu, D. van den Ende, and F. Mugele, "Atomic structure and surface defects at mineral-water interfaces probed by in situ atomic force microscopy," Nanoscale, vol. 8, no. 15, pp. 8220-7, 2016.

[5 1 ] A. Klaassen, F. Liu, D. van den Ende, F. Mugele, and I. Siretanu, "Impact of surface defects on the surface charge of gibbsite nanoparticles," Nanoscale, 2017. 


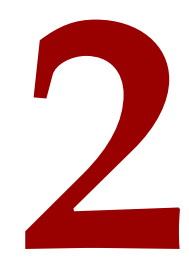

Materials and methods

IN THIS CHAPTER we will further examine the gibbsite clay mineral properties. What is known of gibbsite and what can we contribute to the fundamental knowledge of this material. Besides looking in more detail to the used materials, we will have a more detailed look at the used methods as well. Despite the fact that atomic force microscopy has been around for 30 years, new innovations keep improving the technique, which requires re-evaluation of established procedures. With the introduction of photothermal excitation, our understanding of the effect on measured forces is still little. We will try to improve that in this chapter. We also extract surface charge values from the measured forces, which are different depending on the experimental conditions. In this chapter we will provide a derivation and guide on which procedure to use for the surface charge extraction, as it is relevant for the following chapters. 


\subsection{Introduction to reservoir rocks}

Reservoir rocks are predominantly sedimentary rocks which are porous, permeable, contain sufficient amounts of hydrocarbons and a sealing mechanism from which hydrocarbons can flow [1] The sedimentary rocks can be made of sandstone (quartz sand or arksosic sand), carbonate mud or dolomite. In this thesis we consider the sandstone reservoir rock. Sandstone reservoirs consist mainly of minerals like quartz, feldspar, iron oxides and clay minerals such as kaolinite, illite, montmorillonite and gibbsite. As explained in chapter 1 , the clay content of the rock reservoir plays an important role on the fraction of recovered oil due to its large surface to volume ratio. Since silica and gibbsite are most abundant in the reservoir, we will use them as our model sandstone rock and clay mineral throughout this thesis.

\subsubsection{Silica}

Silicates are rock-forming minerals with predominantly silica anions. They are the largest and most important class of rock-forming minerals [2] Silica (silicon dioxide $\mathrm{SiO}_{2}$ ) can be found in nature as the mineral quartz or in other polymorphs. In mineralogy silicates are classified into 7 major groups according to the structure of their silica anion. Phyllosilicate is one of them and will be discussed in the next section. We use thermal oxidation of silicon wafers to produce thin layers of silicon dioxide.

\subsubsection{Clays}

The definition of a clay has been revised many times since its first formalization in 1546 by Agricola [3]. The most recent definition describes clay as a naturally occurring material composed primarily of fine-grained minerals, which is generally plastic at appropriate water content and will harden when dried or fired. Clays most often consist of phyllosilicates (parallel sheets of silicate tetrahedra) that can contain variable amounts of iron, magnesium, alkali metals, alkine earths, but may contain other materials that impart plasticity and harden when dried or fired. The fine-grained aspect mentioned in the definition cannot be quantified, since there is no universally 
accepted particle size. Geologists and soil chemist use a particle size below $2 \mu \mathrm{m}$, sedimentologists use $4 \mu \mathrm{m}$ and colloid chemists use $1 \mu \mathrm{m}$ [3]

Clays form as a result of chemical weathering of a variety of minerals at the earth's surface and are very common in soils [4]. They are characterized by a layered structure build from tetrahedral silicate sheets $(\mathrm{T})$ and aluminum/magnesium hydroxide octahedral hydroxide sheets $(\mathrm{O})$. A $1: 1$ clay would consist of one of both sheets (TO), like kaolinite, dickite, and antigorite. A 2:1 clay would consist of two silica and one hydroxide sheet (T-O-T), like mica, illite and montmorillonite. The sheets are bonded to each other by hydrogen bonds and van der Waals forces.

The surface morphology of each clay mineral is unique. For example, kaolinite has a plate-like pseudo hexagonal shape whereas illite has a lath-like or fibrous morphology $[\overline{5}, 6]$. Also, the thickness in between clay minerals can vary from $1 \mathrm{~nm}$ for montmorillonite to thousands of nm for kaolinite.

Clay minerals can carry two types of charge, permanent and $\mathrm{pH}$ dependent. The permanent charge can arise from isomorphous substitution of lattice ions or lattice imperfections. $\mathrm{pH}$ dependent charge arises from protonation/deprotonation of surface hydroxyl groups. Generally, it is believed that the basal plane of clay minerals carry a permanent charge, whereas side facets have a $\mathrm{pH}$ dependent charge [7,8]. However, recent research shows that even basal planes of some clay minerals have a $\mathrm{pH}$ dependent charge [9-12].

\subsubsection{Gibbsite}

As mentioned in the introduction, kaolinite is dominantly present in oil rock reservoirs, which makes it an excellent candidate to study in a model system. Kaolinite has an aluminum octahedral hydroxide sheet, which is like gibbsite. Gibbsite can be synthesized reproducibly to yield suspensions of essentially mono-dispersed particles and therefore a good model clay mineral. Gibbsite $\left(\gamma-\mathrm{Al}(\mathrm{OH})_{3}\right)$ is one of the mineral forms of aluminum hydroxide. Its basic structure is formed from stacked sheets of linked octahedrons of aluminum hydroxide. An image of the crystal structure can be found in figure 2.1.

The ratio of the ionic radii favors the octahedral coordination, with $\mathrm{Al}_{3}{ }^{+}$at the cen- 

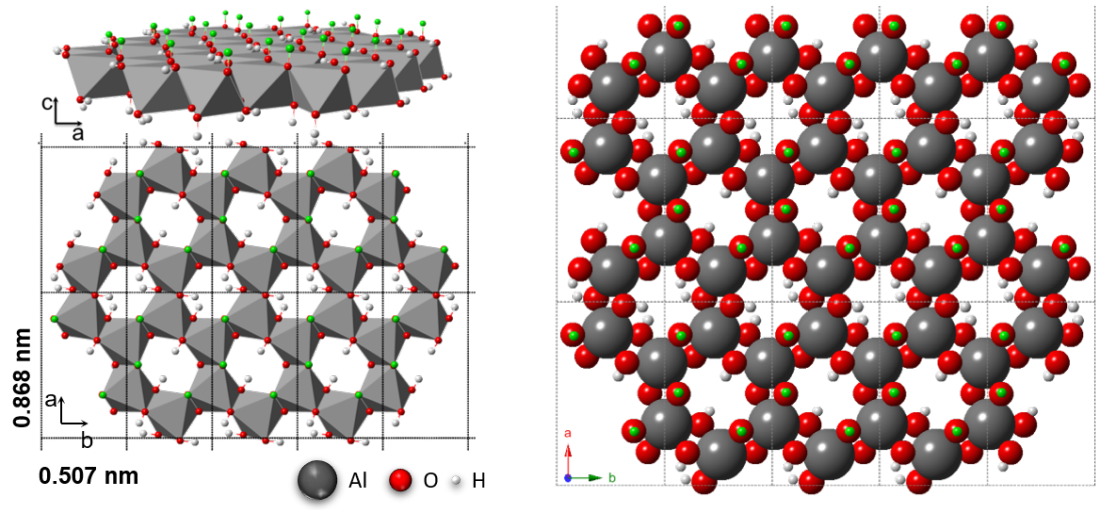

Figure 2.1: a) Gibbsite crystal lattice with given lattice directions $a$ and $b$. The gray, red and white spheres are aluminum, oxygen and hydrogen atoms, respectively.

ter of an octahedron formed by six close packed hydroxide ions $\left(\mathrm{OH}^{-}\right)^{[13]}$. The gibbsite surface unit cell has six chemically inequivalent $\mathrm{Al}_{2}-\mathrm{OH}$ moieties. Simulations suggest that the (de)protonation reactions of these sites have equilibration constants that cover a rather wide range of $\mathrm{pK}_{a}[14]$. Three of the hydroxide ions are located around the central octahedral cavity and point toward the solution. These $\mathrm{OH}$ groups are available for interlayer hydrogen bonding in the bulk gibbsite structure ${ }^{[15]}$ and for hydrogen bonding to adsorbates at the surface $[16-18]$.

In the idealized model of the hydrated alumina surface two types of surface hydroxyl groups can be distinguished (figure 2.1); those coordinated with one aluminum ion and those coordinated by two aluminum ions. The singly coordinated groups are more likely to deprotonate at neutral $\mathrm{pH}$ than the doubly coordinated groups. Therefore, they are more likely to participate in ligand exchange reactions. Since the conception exists that the edge faces consist of singly coordinated groups [19], it is expected that they play an important role in interactions. Parfitt et al. estimated that the basal plane of gibbsite contains approximately 12 hydroxyl groups per square nanometer, while edge planes contain about 4 groups per nanometer [20]. Kummert and Stumm reported a value of 8.5 exchangeable protons per square nanometer [21] based on the isotopic exchange technique of Yates and Healy [22]. 
a)
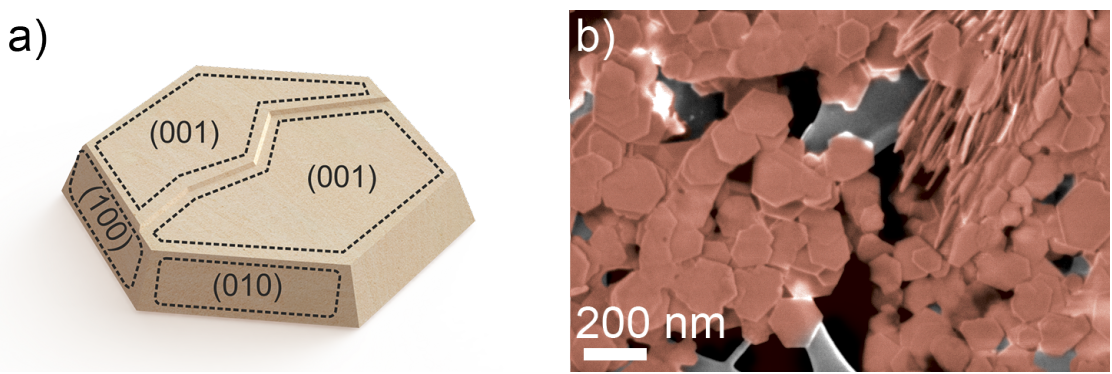

Figure 2.2: a) Cartoon of a gibbsite particle where the crystal planes are depicted. The basal plane (oo1) consists of doubly coordinated hydroxyl groups $\equiv \mathrm{Al}_{2} \mathrm{OH}$ and the edge facets mainly consist of singly coordinated hydroxyl groups $\equiv A l O H$. b) A high resolution scanning electron microscope (HRSEM) image of many gibbsite platelets deposited on carbon conductive tape.

\subsection{Atomic force microscopy}

As explained in chapter 1 , the atomic force microscope (AFM) can be used to measure surface interaction forces. For our particles these interactions are electrostatic in nature and determined by the surface charges on the tip and substrate. Using appropriate modeling we can determine these surface charges from the measured forcedistance curves. This gives us information about how the clay surface reacts with adsorbing molecules and other surfaces.

\subsubsection{Cantilevers}

In AFM a cantilever is used for sensing. A cantilever is a thin beam with a tip mounted on its free end. The tips we use are made of silicon, but can be of a different material, like silicon nitride. Typically cantilevers are 50 to $200 \mu \mathrm{m}$ long, 10 to $40 \mu \mathrm{m}$ wide and 0.5 to $2 \mu \mathrm{m}$ thick. The tip is typically $15 \mu \mathrm{m}$ high and tip radii can range from $1 \mathrm{~nm}$ to $10 \mu \mathrm{m}$. When the tip is in the micron range, we call it a colloidal probe. Some examples of tips can be found in figure 2.3.

For a qualitative analysis of the experimental data, the relation between the measured deflection of the cantilever and the applied force should be known, i.e. the 


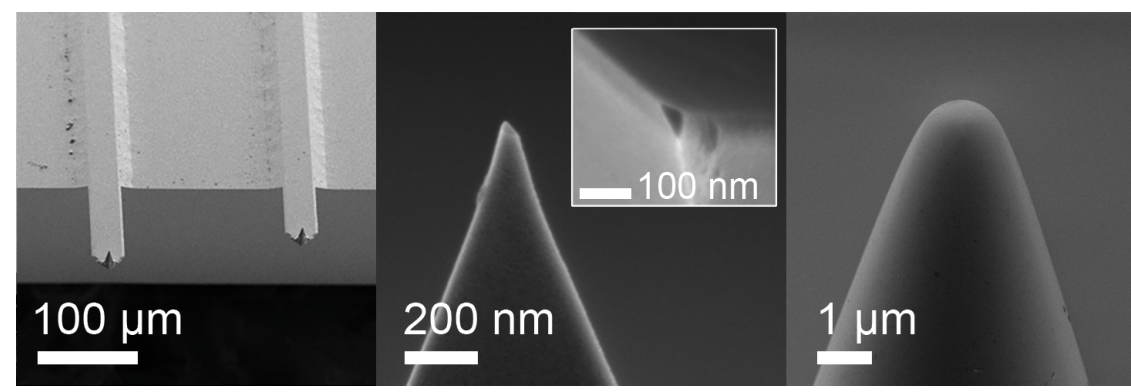

Figure 2.3: High resolution scanning electron microscope images of used cantilevers throughout the thesis. On the left, a chip with several cantilevers having different properties (e.g. spring constant and resonance frequency). In the middle a sharp cantilever tip which has been flattened slightly to increase the tip radius. On the right a colloidal probe with a tip radius of around $750 \mathrm{~nm}$.

spring constant $k_{c}=-\frac{\partial F_{c}}{\partial z}$, or bending stiffness, should be determined via a calibration procedure. The bending stiffness of a cantilever can be determined using Sader's method [23,24]. Sader's method uses the dimensions, resonance frequency and quality factor of the cantilever. However, the specifications from the supplier are often inaccurate and therefore the spring constant is rather determined using the thermal fluctuations [25]:

$$
k_{c}=\frac{k_{B} T}{<z^{2}>}
$$

where $k_{B} T$ is the thermal energy and $<z^{2}>$ is the mean square displacement due to thermal fluctuations.

\subsubsection{Static force spectroscopy}

The deflection from the cantilever is measured with a detection laser beam, i.e. the red beam in figure 1.4, which is reflected from the back of the free end of the cantilever and monitored by the quadrant photodetector. The voltage that is measured by the photodetector is converted to a tip displacement using a calibration procedure. The tip approaches the surface using the z-piezo for as long as the photo detector does not measure a $0.5 \mathrm{~V}$ of tip deflection. From the point where the tip touches the surface (typically around $0.05 \mathrm{~V}$ ) to the $0.5 \mathrm{~V}$ tip deflection, the deflection is linear with the 
z-piezo position. The slope should be -1 when the tip is in hard contact with the surface.

The force can be calculated using the spring constant and measured deflection, since the cantilever acts like a spring.

$$
F=k_{c} z
$$

where $k_{c}$ is the cantilever's spring constant. One downside of using contact mode AFM force spectroscopy is that the signal does not give any information about the dissipation. Furthermore, a snap-in can occur when the attractive force gradient is larger than the spring constant of the cantilever. To overcome this problem stiffer cantilevers can be used. However, for the same force the cantilever will deflect less, resulting in a decrease in sensitivity. This problem was one of the main reasons why dynamic AFM was invented. Using dynamic AFM can overcome the problem of sample damage.

\subsubsection{Dynamic force spectroscopy}

In dynamic force spectroscopy the cantilever is made to oscillate. To oscillate the cantilever, a piezo excitation, a magnetic drive or photothermal excitation can be used. With piezo excitation the base of the cantilever is acoustically driven with a piezoelectric actuator. Magnetic drives require a magnetic coating on the back of the cantilever and a coil which generates an alternating magnetic field. Photothermal excitation uses an intensity modulated laser to periodically heat the base of the cantilever [26]. This requires a cantilever with a back coating of a different metal (typically gold or aluminum). It is based on the bimetallic effect, which means that bending is a result of a difference in thermal expansion coefficient of the coating and cantilever material. The wavelength of this laser (blue) is different from that of the detection laser (red) to prevent interference between the two. The strong advantage of using photothermal excitation with respect to acoustic driving is that the piezo-response spectrum of the cantilever itself is often obscured by additional resonances from the piezo, surrounding liquid and geometry. In figure 2.4 a the 'forest of peaks' in the transfer function of the cantilever is visible, whereas the transfer function with photothermal excitation almost resembles theory (figure 2.4). There are two popular driving schemes for the dynamic force spectroscopy, which are amplitude modulation (AM) 

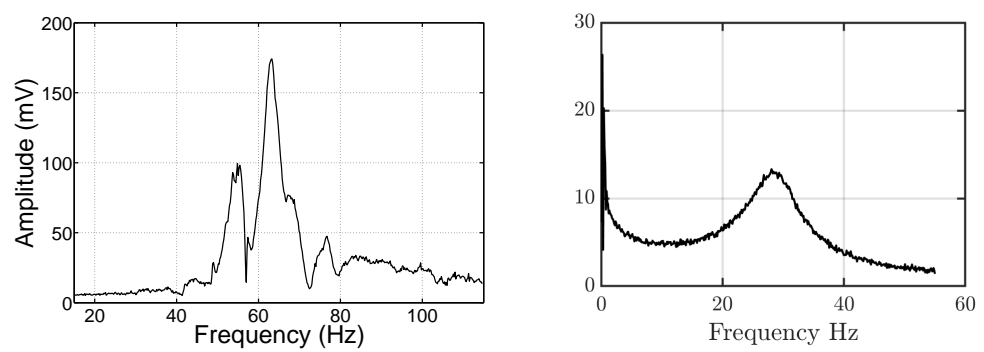

Figure 2.4: A typical frequency response of a cantilever with acoustic driving on the left, and with photothermal excitation on the right. The frequency response using photothermal excitation does not suffer from the 'forest of peaks'.

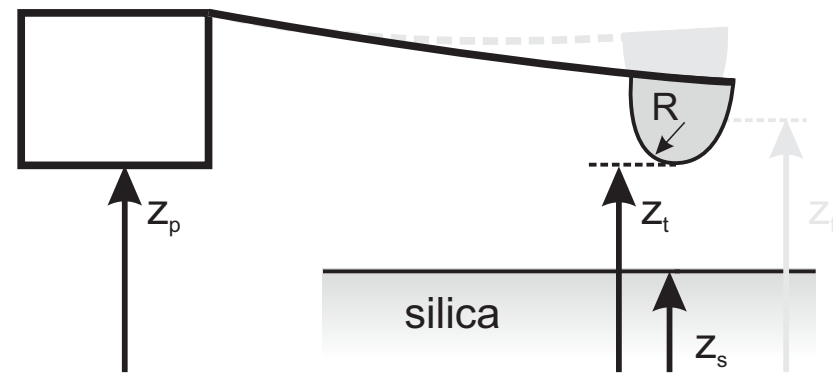

Figure 2.5: Illustration of the cantilever with tip radius $R$, with the total motion $z_{t}$ of the cantilever tip, the sample position $z_{s}$ and piezo distance $z_{p}$. Tip-sample distance is derived from $z_{t s}=z_{p}-z_{t}$

and frequency modulation (FM). With frequency modulation the cantilever is always driven at its actual resonance frequency [27-29]. For this type of modulation two modes exist, constant amplitude (CA) and constant excitation (CE). In FMCA mode the amplitude of the oscillation of the cantilever is kept constant, whereas in FM-CE mode the amplitude of the excitation is kept constant. With amplitude modulation the cantilever is excited at a constant frequency, just below its resonance. The interaction forces measured during the experiment will change the resonance frequency and so the oscillation amplitude at the driving frequency. Therefore, the oscillation amplitude and phase are recorded during the experiment. 
In order to extract any quantitative information from amplitude modulation experiments, a procedure is required to convert the amplitude and phase to interaction forces. The procedure starts with modeling the cantilever dynamics as a simple harmonic oscillator $(\mathrm{SHO})^{[3 \mathrm{O}]}$. The general equation for the system is

$$
m_{c} \ddot{z}=k_{c}\left(z_{t}-z_{T}\right)+F_{\text {drag }}+F_{\text {int }},
$$

where $z_{t}$ is the momentary tip displacement with respect to the average distance $d$, $m_{c}$ the mass of the cantilever and $k_{c}$ its stiffness, while $z_{T}$ is the zero-load tip displacement due to the thermal driving. According to linear response theory, $z_{T}$ can be written, for small thermal variations, as $z_{T}(t)=\int A_{T}\left(t^{\prime}\right) I\left(t-t^{\prime}\right) d t^{\prime}$, or in the frequency domain as $z_{T}(\omega)=A_{T}^{*}(\omega) I_{0}$. The drag force on a sphere with radius $R$ oscillating with frequency $\omega$ in a liquid with density $\rho$ and viscosity $\eta$ is given by [31]

$$
F_{\text {drag }}=-\left(6 \pi \eta R+3 \pi R^{2} \sqrt{2 \eta \rho \omega}\right) \dot{z}-\left(\frac{2}{3} \pi R^{3}+3 \pi R^{2} \sqrt{\frac{2 \eta \rho}{\omega}} \ddot{z}\right),
$$

which we simplify to $F_{d r a g}=-\gamma_{c} \dot{z}-m_{a d d} \ddot{z}$, where $\gamma_{c}$ and $m_{\text {add }}$ are considered to be constant. The interaction force, for small amplitudes, is modeled as

$$
F_{\text {int }}\left(z_{t}, \dot{z}_{t}\right)=F_{\text {int }}\left(z_{e}, o\right)+k_{\text {int }}\left(z_{t}-z_{e}\right)+\gamma_{\text {int }} \dot{z}_{t},
$$

where $F_{\text {int }}\left(z_{e}, 0\right)$ is the equilibrium force at distance $z_{e}, k_{\text {int }}=-\partial F_{\text {int }} / \partial z$ is the interaction stiffness and $\gamma_{\text {int }}$ is the interaction damping. Substituting $F_{\text {drag }}$ and $F_{\text {int }}$ into equation 2.3 results in

$$
m^{*} \ddot{z}_{t}+\left(\gamma_{c}+\gamma_{\text {int }}\right) \dot{z}_{t}+\left(k_{c}+k_{\text {int }}\right) z_{t}=k_{c} z_{T}+k_{\text {int }} z_{e}+F_{\text {int }}\left(z_{e}, o\right),
$$

where $m^{*}=m_{c}+m_{\text {add }}$ is the effective mass of the cantilever.

In equilibrium holds $\ddot{z}_{t}=\dot{z}_{t}=0, z_{T}=z_{T}^{e}$ and $z_{t}=z_{e}$. Applying this to equation 2.4 yields

$$
k_{c} z_{e}=k_{c} z_{T}^{e}+F_{\text {int }}\left(z_{e}, o\right)
$$

Rewriting this for $F_{\text {int }}$ yields

$$
F_{\text {int }}\left(z_{e}, o\right)=k_{c}\left(z_{e}-z_{T}^{e}\right) .
$$

Substituting $k_{c} z_{T}^{e}=F_{\text {int }}\left(z_{T}^{e}\right)$ results in:

$$
F_{\text {int }}\left(z_{e}\right)-F_{\text {int }}\left(z_{T}^{e}\right)=-k_{\text {int }}\left(z_{e}-z_{T}^{e}\right) .
$$




\subsubsection{Force inversion}

To analyze the measured response in terms of the tip-sample interaction, we rewrite equation 2.4 in the frequency domain as:

$$
\left[k_{c}-\omega^{2} m^{*}+i \gamma_{c} \omega\right] z(\omega)+\left[k_{i n t}+i \omega \gamma_{i n t}\right] z(\omega)=k_{c} z_{T}(\omega),
$$

which can be rewritten after dividing the LHS and RHS by $k_{c}$ :

$$
(H(\omega)+K(\omega)) z(\omega)=z_{T}(\omega),
$$

where $H(\omega)=1-\left(\omega / \omega_{\mathrm{o}}\right)^{2}+i\left(\omega / \omega_{\mathrm{o}}\right) Q^{-1}$ and $K(\omega)=\left(k_{\text {int }}+i \omega \gamma_{\text {int }}\right) / k_{c}$ with $m^{*}=k_{c} / w_{\mathrm{o}}^{2}$ and $Q_{0}=k_{c} /\left(\gamma_{c} \omega_{\mathrm{o}}\right)$. Rewritten in terms of $z$ :

$$
\begin{aligned}
z(\omega) / z_{T}(\omega) & =1 /[H(\omega)+K(\omega)] \\
z_{\infty}(\omega) / z_{T}(\omega) & =1 / H(\omega)
\end{aligned}
$$

Now eliminating $z_{T}(\omega)$ from above equations and rewriting for $K(\omega)$ leads to

$$
K(\omega)=H(W) \frac{z_{\infty}(\omega)-z(\omega)}{z(\omega)}
$$

Writing $z(\omega)=A e^{i \varphi}$ and $z_{\infty}(\omega)=A_{\infty} e^{i \varphi} \infty$ we get:

$$
K(\omega)=\frac{H\left(A_{\infty}-A e^{i\left(\varphi-\varphi_{\infty}\right)}\right.}{A e^{i\left(\varphi-\varphi_{\infty}\right)}}
$$

Before we can use equation 2.8 to determine $k_{\text {int }}$ and $\gamma_{\text {int }}$ from $A e^{i \varphi}$, we need to calibrate $k_{c}, \gamma_{c}$ and $m^{*}$. The spring constant is determined from the thermal noise spectrum as explained in the first section. The mass $m^{*}$ and damping $\gamma_{c}$ are obtained from the resonance frequency $\omega_{\mathrm{o}}$ and quality factor $Q$. Using the ratio $A / A_{\infty} e^{i\left(\varphi-\varphi_{\infty}\right)}$ we can rewrite equation 2.8 to:

$$
\frac{A}{A_{\infty}} e^{i\left(\varphi-\varphi_{\infty}\right)}=\frac{k_{c}-m^{*} \omega^{2}+i \gamma_{c} \omega}{k_{c}-m^{*} \omega^{2}+i \gamma \omega+k_{i n t}+i \gamma_{i n t} \omega}
$$

from which we obtain the inversion formulas:

$$
k_{\text {int }}=k_{c}\left[1-\left(\omega / \omega_{\mathrm{o}}\right)^{2}\right] \frac{A_{\infty} \cos \left(\varphi-\varphi_{\infty}\right)-A}{A}+\gamma_{c} \omega \frac{A_{\infty} \sin \left(\varphi-\varphi_{\infty}\right)}{A}
$$

and

$$
\gamma_{i n t}=\gamma_{c} \frac{A_{\infty} \cos \left(\varphi-\varphi_{\infty}\right)-A}{A}-k_{c}\left[1-\left(\omega / \omega_{\circ}\right)^{2}\right] \frac{A_{\infty} \sin \left(\varphi-\varphi_{\infty}\right)}{A}
$$



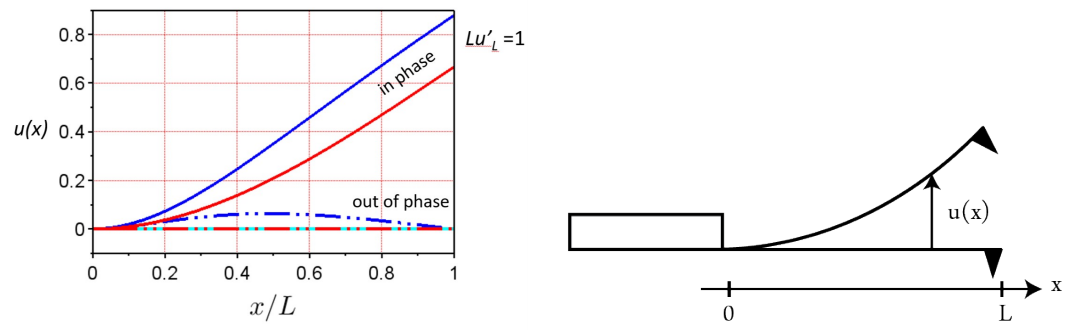

Figure 2.6: Result of driving forces on the shape of the cantilever. In red and blue the shape of a cantilever when driven acoustically (red) and thermally (blue) plotted versus normalized cantilever length. Solid lines represent the shape of the cantilever and dotted lines the phase of the oscillation. The resonance frequency of the cantilever in this example is $16 \mathrm{kHz}$.

\section{Photothermal excitation}

The approach from the previous section is not fully correct, because under photothermal driving the frequency response of deflection and displacement behaves differently than modeled here. Furthermore, the hydrodynamic interaction does not take place at the tip only, but along the whole beam of the cantilever [23]. Despite these difficulties we can give a better approximation.

We start with a correction of the cantilever shape as a function of frequency. In figure 2.6 the difference between acoustically (red) and photothermally (blue) excited cantilevers is shown. The solid lines show the shape of the cantilever when excited in phase of the first mode and the dotted lines when excited out of phase. From the graph it is clear that the slope $\left(L u_{L}^{\prime}\right)$ for both is similar, but the local distortion itself is not. This results in the fact that the total displacement of the tip is different from its deflection.

The influence of the driving laser on measured quantities is not well understood yet. Not optimal positioning of the driving laser might introduce unwanted secondary oscillations and therefore changing the apparent stiffness of the system. Positioning of the excitation laser is important too, because the resulting oscillation amplitude 

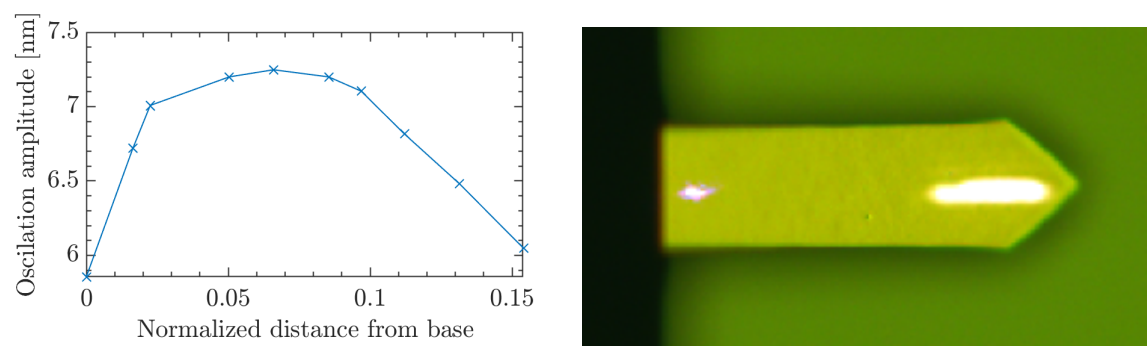

Figure 2.7: Cantilever oscillation amplitude as function of the position of the excitation laser from the base. The position is normalized by the length $(225 \mu \mathrm{m})$ of the cantilever. On the right an example of a cantilever excited by a blue laser (left bright dot) and the resulting amplitude is measured by the red laser (bright stripe on the right). The position of the excitation laser can be changed with respect to the red laser.

can drop by $20 \%$ over $30 \mu \mathrm{m}$ (figure 2.7).

\subsection{Physical forces experienced by AFM tip}

In an AFM measurement, the tip is usually made of silicon and its surface is covered by a few nanometers of native silica. In an aqueous solution the tip becomes charged due to the deprotonation of silanol groups $(\mathrm{SiOH})^{[32]}$. When performing force spectroscopy, the resulting electrostatic interactions should be taken into account. To do so, first is shown how the DLVO theory is used to account for the tip geometry and then it is shown how to solve the Poisson-Boltzmann equation with proper boundary conditions.

\subsubsection{DLVO}

In direct force measurements, two surfaces are brought into close contact. The interaction forces that arise between these two surfaces can be described using the DLVO theory [33,34]. This theory assumes that the interaction is a sum of the van der Waals force and the electric double layer forces. The double layer forces mainly 
a)

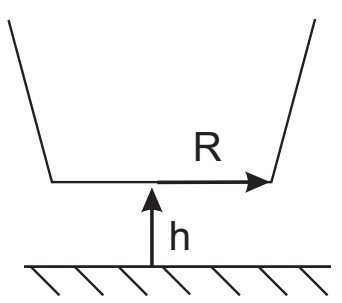

b)

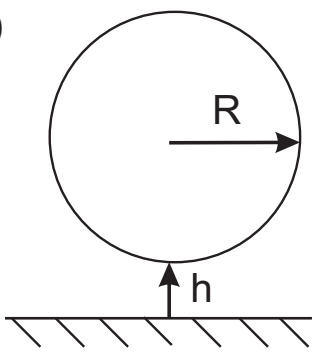

Figure 2.8: Examples of tip geometries used in AFM force spectroscopy. a) plate-plate geometry. b) sphere-plate geometry.

result from the excess osmotic pressure of the inhomogeneous counter ion distribution in the EDL, i.e. the ions in the diffuse layer $\left(\sigma_{D}\right)$ that compensate the intrinsic surface charge density $\left(\sigma_{\mathrm{o}}\right)$ on the surface and the immobilized ions in the Stern layer $\left(\sigma_{S}\right)$.

$$
\Pi_{d i s}=\Pi_{v d W}+\Pi_{d l}
$$

For two semi-infinite parallel plates, the van der Waals part can be described by:

$$
\Pi_{v d W}=-\frac{A_{H}}{6 \pi z^{3}}
$$

where $A_{H}$ is the Hamaker constant and $z$ the distance between the parallel plates. The double layer pressure $\Pi_{d l}$ has two components, the osmotic pressure and Maxwell stress:

$$
\Pi_{d l}=\underbrace{2 k_{B} T \sum_{i}\left[n_{i}-n_{i(\infty)}\right]}_{\text {osmotic }}-\underbrace{\frac{\mathcal{E} \mathcal{E}_{\mathrm{o}}}{2}(\nabla \psi)^{2}}_{\text {Maxwell }} .
$$

The measured force gradient depends on the tip geometry. When the tip radius is too small, the tip is flattened to increase the tip radius (and interaction area). The interaction area is then approximated as a flat disk with radius $R$ (figure 2.8). When lateral resolution is not of interest, a colloidal probe can be used. In that case, the interaction area is approximated as a sphere with radius $R$. 


\section{Plate-plate}

In the case of the plate-plate geometry (figure $2.8 \mathrm{a}$ ) the interaction force $F_{\text {int }}$ can be described by:

$$
F_{\text {int }}=\Pi_{d i s}(z) \pi R^{2}
$$

where $R$ is the radius of the flat contact area of the tip, $z$ the distance between the tip and sample and $\Pi_{\text {dis }}$ the excess pressure between tip and sample. This excess pressure is the sum of the van der Waals pressure $\left(\Pi_{v d W}\right)$ and the double layer component $\left(\Pi_{d l}\right)^{[35]}$. Since from the force inversion equation we actually get the $k_{i n t}$ instead of the $F_{\text {int }}$, we relate the disjoining pressure with the $k_{\text {int }}$ with

$$
\int_{h}^{\infty} k_{\text {int }}(z) d z=\pi R^{2} \Pi_{d i s}(h) .
$$

\section{Sphere-plate}

In the case of a sphere plate geometry the interaction force is described by

$$
F_{\text {int }}=\int_{0}^{\infty} \Pi_{d i s}(z(r)) 2 \pi r d r
$$

To evaluate the integral the Derjaguin approximation $[36]\left(z(r)=h+r^{2} /(2 R)\right)$ can be used, which holds under the condition $h<<R$. This results in $r d r=R d z$ on which we can rewrite equation 2.3.1 to

$$
\frac{F_{\text {int }}}{R}=2 \pi \int_{h}^{\infty} \Pi_{d i s}(z) d z .
$$

From the measurements we retrieve the $k_{i n t}$ instead of the $F_{i n t}$, so we finally obtain

$$
\frac{k_{i n t}}{2 \pi R}=\Pi_{d i s} .
$$




\section{Electric double layer}

Various models are used to describe the electric double layer (EDL) and the Stern layer, like the Gouy-Chapman model (GCM), the Basic Stern model (BSM) and the triple layer model (TLM). [37,38,22,39]. All these models describe the double layer slightly differently depending on to what extent the counter ions are able to approach the substrate. In the GCM all ions can reach the substrate so the diffuse layer extends from the substrate into the bulk liquid. In the BSM only the protons can reach the substrate while the other ions, due to finite size effects, can only approach the substrate up to a certain distance, which correlates with the size of the ion. Hence, in the Stern layer no ions are present. In the TLM, the 'o-plane' is the plane where charge is generated through (de-) protonation reactions on the surface, often called the intrinsic surface charge. The ' $\beta$-plane' is located at the Stern layer, the first layer of adsorbed ions. The 'd-plane' is located at the start of the diffuse layer. The different types of charge in the EDL are relevant for different physical phenomena. When electrostatic forces are important, as in case of electrophoretic mobility and colloidal stability, the diffuse layer charge plays an important role. Whereas in surface conduction and other surface relevant processes, the Stern layer charge is more relevant.

As mentioned in chapter 1 , the diffuse layer has a characteristic thickness known as the Debye length $\kappa$. The thickness of this layer depends on the ionic strength $\left(I_{\infty}\right)$ of the solution, which is clear from the definition of the Debye length:

$$
\kappa=\sqrt{\frac{2 I_{\infty} e^{2}}{\varepsilon \varepsilon_{0} k_{B} T}},
$$

where $\mathcal{E}_{\mathrm{O}}$ is the dielectric permittivity of water, $k_{B}$ the Boltzmann constant and $T$ the temperature. The charge density in the diffuse layer decreases to zero in the bulk. The charge density of the ionic species present in the diffuse layer obey the Boltzmann relation:

$$
n_{i}(\psi)=n_{\infty} \exp \left[\frac{-e Z_{i}(\psi)}{k_{B} T}\right],
$$

where $\psi$ is the local potential. The surface potential and charge relation is governed by the Poisson equation:

$$
\nabla^{2} \psi=\frac{-\rho}{\mathcal{E}_{\mathrm{o}}},
$$


where $\rho=\sum_{i} e Z_{i} n_{i}(\varphi)$ is the charge density. Combining equation 2.16 and 2.17 results in the Poisson Boltzmann equation:

$$
\nabla^{2} \psi=\frac{-e}{\mathcal{E}_{\mathrm{o}}} \sum_{i} Z_{i} n_{i}(\psi) \exp \left[\frac{-e Z_{i} \psi}{k_{B} T}\right]
$$

The surface charge and surface potential are not known and need to be determined. For an electric double layer at a single solid electrolyte interface this equation reduces to Grahame's equation [40]. Grahame's equation relates the total diffuse layer charge density and the potential drop in a double layer through:

$$
\sigma_{d}=\sqrt{{ }_{9 I_{\infty} k_{B} T \varepsilon \varepsilon_{\mathrm{o}}}} \sinh \frac{e \psi_{d}}{2 k_{B} T} .
$$

\section{Charge regulation}

Double layer forces are described using Poisson Boltzmann (PB) theory, where the solvent and the ion distribution are modeled as continuous media. This model can be extended by incorporating, for example, ion-ion correlations, specific adsorption, or finite size effects of the solvent and ions [41-46]. To calculate the excess pressure between tip and substrate, one solves the PB equation for the electric potential. The surface charge or surface potential of both substrates determine the boundary conditions for the $\mathrm{PB}$ equation. Classically, one supposes a constant surface charge or a constant potential when the interaction distance is varied. However, when the distance between the two charged surfaces becomes small and the two double layers start to overlap (figure 2.9), the resulting potential distribution will modify the ion distribution near the surfaces. This affects the (de-) protonation at the surfaces as well as the ion ad- or desorption in the Stern layers, and so the resulting surface charge densities. The redistribution of the surface charge and diffuse layer potential when the separation distance is varied, is called charge regulation [47-50]. Due to charge regulation the surface potential and surface charge become distance dependent which complicates the determination of the right boundary conditions for the PB equation.

Despite the fact that charge regulation has been well-known $[51,40,39,48]$, it is not always implemented in the direct force measurement. Borkovec et al. [54] used charge 

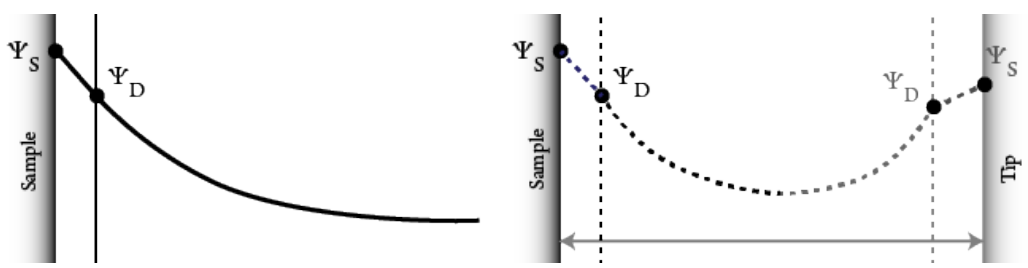

Figure 2.9: The surface potential over the Stern Layer and the diffuse layer. Diffuse layers start to overlap in the presence of a second charged surface.

regulation on colloidal probe data and were able to extract diffuse layer charges for latex particles. Zhao et al. [52] used charge regulation on force distance curves (data recorded with sharp tips at $\mathrm{pH} 6$ and several sodium chloride conditions) together with a surface complexation model.

We use surface complexation models that describe the surface to construct the CR boundary condition $[48,53,49,52]$ for equation 2.18 . We consider a surface site $S H$ that can deprotonate to produce a negatively charged site $\mathrm{S}^{-}$. The mass action law that the reaction equilibrium $\mathrm{SiOH} \rightleftharpoons \mathrm{SiO}^{-}+\mathrm{H}^{+}$relates to the site densities $\{\mathrm{SiOH}\}$ and $\left\{\mathrm{SiO}^{-}\right\}$is:

$$
\left\{\mathrm{SiO}^{-}\right\}\left[\mathrm{H}^{+}\right]_{s}=K_{H}\{\mathrm{SiOH}\},
$$

where the braces ' \{\} ' indicate surface concentrations and square brackets indicate volume concentrations. The equilibrium constant $K_{H}$ has a corresponding $p K$ value that is described by $p K_{H}=-\log K_{H}$. The deprotonated surface group may adsorb a counter-ion from the solution to form a surface complex, which can be described by the following chemical reaction:

$$
\mathrm{SiOC} \rightleftharpoons \mathrm{SiO}^{-}+\mathrm{C}^{+}
$$

This chemical reaction has an equilibrium constant described by

$$
K_{C}=\{\mathrm{SiOC}\}\left[C^{+}\right]_{d} /\{\mathrm{SiOC}\}
$$

Since the total surface sites of surface $S$ is fixed by the geometry and surface chemistry, the total number density of surface groups $\Gamma$ can be written as:

$$
\Gamma=\{\mathrm{SiOH}\}+\left\{\mathrm{SiO}^{-}\right\}+\{\mathrm{SiOC}\} .
$$


Equation 2.19, 2.20 and 2.21 can be rewritten in matrix form to obtain:

$$
\left(\begin{array}{ccc}
1 & 1 & 1 \\
{\left[H^{+}\right]_{s}} & -K_{H} & 0 \\
{\left[C^{+}\right]_{d}} & 0 & -K_{C}
\end{array}\right)\left(\begin{array}{l}
\left\{\mathrm{SiO}^{-}\right\} \\
\{\mathrm{SiOH}\} \\
\{\mathrm{SiOC}\}
\end{array}\right)=\left(\begin{array}{c}
\Gamma \\
0 \\
0
\end{array}\right)
$$

The surface charge density $\sigma$ is determined by the number of negatively charge surface sites, resulting in:

$$
\sigma=-e\left\{\mathrm{SiO}^{-}\right\}=-\frac{e \Gamma}{1+\left[H^{+}\right]_{s} / K_{H}}
$$

The local proton concentration $\left[\mathrm{H}^{+}\right]_{s}$ and $\left[\mathrm{C}^{+}\right]_{d}$ are assumed to follow the Boltzmann distribution

$$
\begin{aligned}
& {\left[H^{+}\right]_{s}=\left[H^{+}\right]_{\infty} e^{-e \psi_{s} / k_{B} T}} \\
& {\left[C^{+}\right]_{d}=\left[C^{+}\right]_{\infty} e^{-e \psi_{d} / k_{B} T}}
\end{aligned}
$$

Here, $\psi_{s}$ is the surface potential. The surface charge is proportional to the potential drop across the Stern layer, leading to:

$$
C_{s}=\sigma_{s} /\left(\psi_{s}-\psi_{d}\right)
$$

Resulting in the CR boundary condition:

$$
\sigma=f\left(\psi_{s} ; K_{H}, K_{C}, \Gamma,\left[H^{+}\right]_{\infty} ;\left[C^{+}\right]_{\infty}, C_{s}\right),
$$

where $\Gamma,\left[H^{+}\right]_{\infty},\left[C^{+}\right]_{\infty}$ and $C_{s}$ are given constants and $K_{H}$ and $K_{C}$ are in most cases the fit parameters. The extracted surface charge $\sigma$ is essentially the net surface charge, i.e. the opposite of the diffuse layer charge. Note that, for example for silica at $\mathrm{pH}_{7}$, the bare surface is negative and the Stern layer with the diffuse layer balance this. Therefore, the effective surface charge is negative $\left(\sigma_{\mathrm{o}}+\sigma_{S}=-\sigma_{D}<\mathrm{o}\right)$.

\subsubsection{Surface charge calculation}

With a surface complexation model the diffuse layer charge can be extracted accurately, but also equilibrium constants ( $\mathrm{pK}$ values) can be determined. However, the 


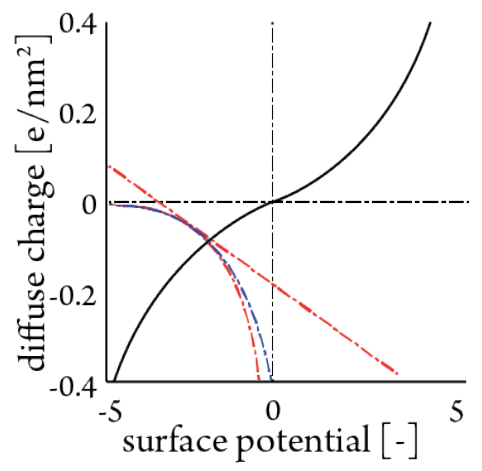

Figure 2.10: Surface charge vs surface potential. Grahame equation in black, surface charge according to $2 \mathrm{pK}$ model in blue and the linear and exponential fit for charge in dashed red lines.

problem with $\mathrm{pK}$ modeling is that the correct surface reactions are unknown. Additionally, for a limited dataset the equilibrium constants from the fit are not necessarily unique $[52]$. Furthermore, because the equilibrium constants are not always of primary interest, we suggest a different approach, by separating the diffuse layer charge and potential calculations from the chemical modeling by implementing a generic differential surface capacitance. This approach was first introduced by Carnie et al. [49], and later adapted by Borkovec and coworkers [54,55]. Where Borkovec and coworkers $[56]$ use a linear parametrization, we use for the silica surface an exponential relation. In figure 2.10 a visual representation of the exponential fit. The black curve is the Grahame equation, and where the blue solid line crosses the black line is the surface charge. The red dashed lines are a linear and exponential fit of the surface charge.

$$
q_{s}=-\exp \left(\psi_{s}-\psi_{f}\right)
$$

where $q_{s}=\sigma_{s} / \sigma_{*}$ and $\psi_{s}=q_{e} \varphi_{s} / k_{B} T ; \sigma_{*}=2 q_{e} I_{\infty} \kappa^{-1}$ with $q_{e}$ the electron charge, $I_{\infty}$ the ionic strength of the bulk solution and $\kappa^{-1}$ the corresponding Debye length. The dimensionless potential $\psi_{f}$ is left as a fitting parameter. We call this expression the exponential surface charge (ESC) model. It is used to determine the boundary values for $\psi$ and $\sigma$ at the tip and the substrate when solving the Poisson Boltzmann 
equation for the potential in the electrolyte film between tip and substrate. Once
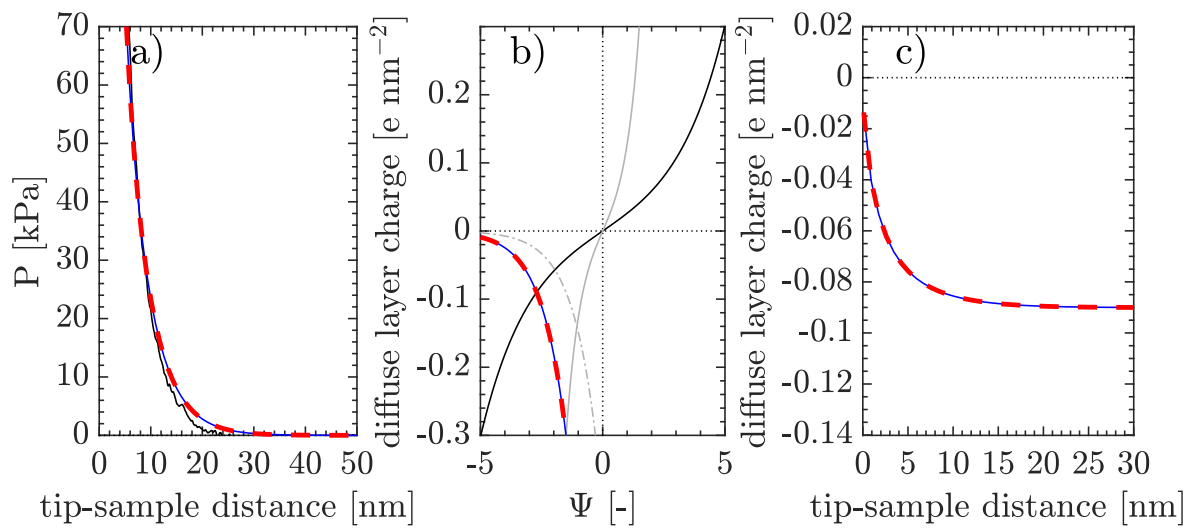

Figure 2.1 1: (a) Pressure-distance curves for the silica-silica interface in a $4 \mathrm{mM} \mathrm{NaCl} p \mathrm{H}$ 9 solution. In black, the recorded experimental curve. In blue the curve resulting from the $2 p K$ model fit $\left(\mathrm{pK}_{\mathrm{H}}=10.0, \mathrm{pK}_{\mathrm{C}}=-5.1, \sigma=-0.085 \mathrm{e} / \mathrm{nm}^{2}\right)$. In red the result of the charge fitting using the ESC model. (b) The net surface charge vs the surface potential. The black solid line is a solution to the Grahame equation, in blue the net surface charge according to the $2 p K$ model while the dashed red line represents the ESC curve. The gray curves are a result of a higher cation concentration. (c) The calculated diffuse layer charge as function of separation distance. The blue line is the net surface charge according to the $2 p K$ model and the red circles are the result of the ESC model fit.

the electric potential is known, we can calculate the excess pressure in the electrolyte film from:

$$
\Pi(h)=I_{\infty} k_{B} T\left\{4 \sinh ^{2}(\psi / 2)-\kappa^{-2}\left(\frac{d \psi}{d z}\right)^{2}\right\}_{z=h / 2}^{+} \Pi_{v d W}(h)
$$

which holds for a 1-1 electrolyte. Here $\Pi_{v d W}=-A_{H} / 6 \pi h^{3}$ is the van der Waals contribution and $h=z_{t s}$ is the distance between tip and substrate. The first term in last expression, $4 I_{\infty} k_{B} T \sinh ^{2}(\psi / 2)$, represents the osmotic contribution to the pressure and the second term, $-I_{\infty} k_{B} T \kappa^{-2}(d \psi / d z)^{2}$, the electric or Maxwell stress contribution. Note that these terms depend on the distance $z$ from the substrate in the electrolyte film, but their sum is independent of $z$. For a spherical tip with 
radius $R$ the excess pressure can be related to the interaction stiffness $k_{i n t}$, using the Derjaguin approximation in equation 2.15 .

Using this approximation, the force can be calculated by integrating the interaction stiffness $F_{\text {int }}(h)=\int_{h}^{\infty} k_{\text {int }}(z) d z$. Figure 2.11 a shows amongst others the experimentally obtained $\Pi(h)$ curve (black curve). To subtract the van der Waals contribution from this pressure curve, we need a value for the Hamaker constant $A_{H}$, which we take from literature: $A_{H}=0.65 \times 10^{-20} \mathrm{~J}[57]$. For large separations the charge in the diffuse layer $\sigma_{d l}$ is given by the Grahame equation (equation 2.3.1), which can be derived by equating the expression between the curly brackets in equation 2.25 to zero. The black line in figure $2.11 \mathrm{~b}$ shows this dependence. On the other hand the total charge on the substrate $\sigma_{s}$ is dictated by charge regulation, see equation 2.24 and the blue curve in figure $2.11 \mathrm{~b}$. As charge neutrality dictates that these charges should be equal, the diffuse layer charge $\sigma_{d l}$ is determined by the crossing of the blue curve with the black Grahame curve. At finite distance between the substrate and tip there arises an excess osmotic pressure in the film that modifies the Grahame equation (the expression between the curly brackets in equation 2.25 becomes positive), causing a reduction of $\left|\sigma_{d l}\right|$, as shown in figure $2.11 \mathrm{c}$. The plateau value of this curve corresponds with the value of $\sigma_{d l}$ at the crossing point in figure $2.11 \mathrm{~b}$.

When the $\mathrm{pH}$ increases, the blue curve in figure 2.1 $\mathrm{b}$ shifts leftwards, causing the intersection with the black Grahame curve to shift down, meaning a larger negative charge. The concentration dependence is less obvious. On the one hand due to the increasing concentration of the cations, $\left[\mathrm{C}^{+}\right]$, the equilibrium should shift toward a higher cation adsorption. In figure $2.11 \mathrm{~b}$, this corresponds to a shift rightwards of the blue curve (gray dashed curve). But on the other hand the Debye length (which is hardly affected by $\mathrm{pH}$ variation) decreases. This steepens the solution of the Grahame equation (black solid curve to the gray solid curve) in figure $2.11 \mathrm{~b}$. So the adsorption is reduced. The net effect is a gradual increase of the net surface charge with concentration as can be observed from the downward shift in the crossing point of the two gray curves. 


\subsubsection{Accuracy and error analysis}

Charge fitting routine To validate our ESC approach we also generated some synthetic force-distance curves by calculating the interaction force from a $2 \mathrm{pK}$ model. The parameters of this model have been listed in Table 3.2. Taking the experimental curve, measured for a $10 \mathrm{mM} \mathrm{NaCl} \mathrm{pH} 6$ solution (black line in figure 2.11 a) as a reference, we first determine the optimal values for $\mathrm{p} K_{H}$ and $\mathrm{p} K_{C}$ by fitting the forcedistance curve with the $2 \mathrm{pK}$ model. Here we take the site density of surface groups on a silica substrate equal to $\Gamma=8 \mathrm{~nm}^{-2}$ [57]. The fit from the $2 \mathrm{pK}$ model (blue) to the experimental curve (black) for $10 \mathrm{mM} \mathrm{pH} 6 \mathrm{NaCl}$ has been plotted in figure 2.11 a). The fit values are shown in the column marked with $2 \mathrm{pK} /$ exp. We also fit the experimental force-distance curve using the ESC model. The obtained values are shown in the column marked by ESC/exp. Next, we use the $\mathrm{pK}$ values from the $2 \mathrm{pK}$ model fitting to generate a force-distance curve which serves as an 'experimental' input for the ESC model. The results from this fitting are shown in the column marked with $\mathrm{ESC} / 2 \mathrm{pK}$ and the curve for $10 \mathrm{mM} \mathrm{pH} 6 \mathrm{NaCl}$ has been plotted in figure $2.11 \mathrm{a}$ (red dashed line). In figure $2.11 \mathrm{~b}$ the net surface charge versus surface potential has been plotted, where the red curve is the fitted exponential approximation around the intersection point with the Grahame equation (black line). The blue curve represents the $\sigma(\varphi)$ relation as calculated from the $2 \mathrm{pK}$ model, using the fitted values for $\mathrm{p} K_{H}$ and $\mathrm{p} K_{C}$. Figure $2.11 \mathrm{c}$ shows the diffuse layer charge as function of the tip-sample distance calculated by fitting the ESC model tot the $2 \mathrm{pK}$ force data (open red circles) compared with the net surface charges, directly calculated with the $2 \mathrm{pK}$ model (full blue line). The agreement between the two curves is good, the deviations are $0.3 \%$ or less, as can be concluded from Table 2.1, where the calculated 'diffuse layer charges' have been listed for various concentrations and $\mathrm{pH}$ values. This means that using the ESC model is able to closely match the results from the $2 \mathrm{pK}$ model. The advantage is that for the ESC model no prior knowledge of the surface chemistry is needed or assumptions have to be made about the surface. This model will be used in Chapter 3 to retrieve diffuse layer charges from the experiments.

Summary and conclusion For the silica-silica system we studied, all obtained diffuse layer charges match within $1 \%$ with the values determined from the $2 \mathrm{pK}$ surface complexation model. Furthermore, the values fitted to the theoretical pressuredistance curves agree also within $1 \%$ of these values. In spite of this success, also 


\begin{tabular}{r|ccc|ccc|ccc}
$\mathrm{mM}$ & \multicolumn{3}{|c|}{$\mathrm{pH} 4$} & \multicolumn{3}{|c|}{$\mathrm{pH} 6$} & \multicolumn{3}{c}{$\mathrm{pH} 9$} \\
\hline model: & $2 \mathrm{pK}$ & $\mathrm{ESC}$ & $\mathrm{ESC}$ & $2 \mathrm{pK}$ & $\mathrm{ESC}$ & $\mathrm{ESC}$ & $2 \mathrm{pK}$ & $\mathrm{ESC}$ & $\mathrm{ESC}$ \\
curve: & $\exp$ & $\exp$ & $2 \mathrm{pK}$ & $\mathrm{exp}$ & $\exp$ & $2 \mathrm{pK}$ & $\mathrm{exp}$ & $\exp$ & $2 \mathrm{pK}$ \\
\hline 1 & -0.02 & -0.02 & -0.02 & -0.05 & -0.05 & -0.05 & -0.07 & -0.07 & -0.07 \\
4 & -0.04 & -0.04 & -0.04 & -0.07 & -0.07 & -0.07 & -0.09 & -0.09 & -0.09 \\
10 & -0.06 & -0.06 & -0.06 & -0.08 & -0.08 & -0.08 & -0.10 & -0.10 & -0.10 \\
40 & -0.09 & -0.09 & -0.09 & -0.11 & -0.11 & -0.11 & -0.14 & -0.14 & -0.13 \\
100 & -0.15 & -0.15 & -0.15 & -0.19 & -0.19 & -0.19 & -0.22 & -0.22 & -0.22
\end{tabular}

Table 2.1: Diffuse layer charge in $\mathrm{e} / \mathrm{nm}^{2}$ for several concentrations and $\mathrm{pH}$ values. The row labeled with model indicates the model used to fit the type of curve (experimental or generated with $2 \mathrm{pK}$ model) shown in the row labeled curve. So the first column shows the diffuse layer charge values that are obtained from experimental curves with the $2 \mathrm{pK}$ model.

in this model solvent and ion distributions are described as a continuous medium, which for sure is questionable at short length scales. A sign of these short range forces might be visible in figure $3.3 \mathrm{~b}$ and $\mathrm{c}$, where deviations in the interaction stiffness occur between various measurements at distances below $2 \mathrm{~nm}$. For instance, Fleharty et al. report that the chemical equilibrium between surface reactive groups and the potential determining ions strongly depends on the precise molecular structure of the solution near the charged interface ${ }^{[43]}$. They argue that a physically adequate analysis of the electric double layer should include charge regulation as well as a full account of the solvent contribution to the solution structure.

\subsection{Principal component analysis}

The interaction forces measured on silica and gibbsite in the aqueous divalent electrolyte solutions are so small that in most electrolyte conditions the force is in the range of the noise. In order to extract any quantitative or qualitative data, the signal to noise ratio (SNR) needs to be increased. Averaging can be applied to increase the SNR, where the SNR increases proportionally to the square root of the number of experiments. During a typical force spectroscopy experiment, $32 \times 32$ approaches are recorded. This number of approaches gives plenty of room for averaging, however, 

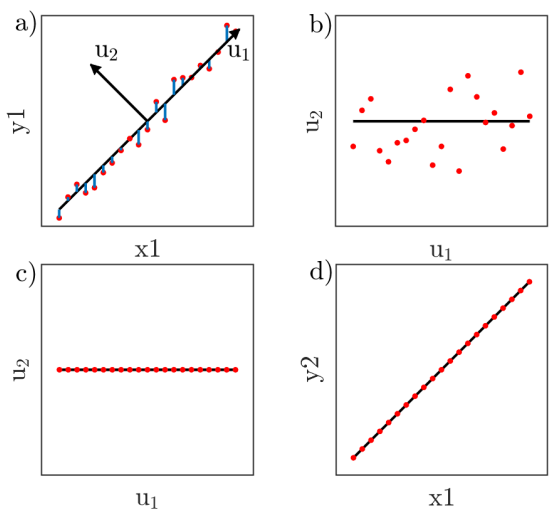

Figure 2.12: a) A $2 D$ data set with random noise with principal component 1 and 2 indicated. b) The data from a) transformed to their principle components. c) The projection of the data on PC 1 becomes the data. d) Transforming back to the original coordinate system.

this will partially sacrifice the lateral resolution of the measurement.

Another method to increase the $\mathrm{S} / \mathrm{N}$ ratio is principal component analysis (PCA). PCA is a powerful statistical method that uses orthogonal transformations to convert a set of possibly correlated variables into a set of values of linearly uncorrelated variables called the principal components. PCA tries to find a lower dimensional subspace such that it maximizes the variances of the projected data $\left(\sum(x-u)^{2}\right)$ and minimizes the mean squared distance between data and their projections. In figure 2.12a this is shown for a line with random noise added, so in this example we reduce $x^{(i)} \in R^{2}$ to $x^{(i)} \in R^{1}$. The variance is maximized by making sure that the projections are as spread out as possible. The mean squared errors are minimized by making the sum of the blue lines as small as possible. Transforming the data to the new coordinate system, figure $2.12 \mathrm{~b}$, shows the correlation in the data. The 'noise' can be removed by using the projection of the data on the $u_{1}$ axis, which results in the data set shown in figure 2.12 c. When the projection is transformed back to the $x-y$ coordinate system, figure $2.12 \mathrm{~d}$, the data is cleaned from noise. This is in basic terms how PCA helps reduce noise in data. It is especially powerful for large datasets and in the case of the force maps we have plenty of data for the PCA to work. 
For PCA to work in the case of AFM data, the amplitude and phase data need to be defined at the same $\mathrm{z}$-distance intervals. This is not true for all recorded data. Therefore, we use interpolation to map all the amplitude and phase data on a unique $\mathrm{z}$-axis with values on a regular interval. This creates a matrix where the amplitude and phase data are defined for each point on the $\mathrm{z}$-distance axis.

$$
x_{i}=\left(\begin{array}{c}
a_{i}^{1} \\
a_{i}^{2} \\
\cdots \\
a_{i}^{k}
\end{array}\right) \text { and } x_{i}^{T}=\left(a_{i}^{1}, a_{i}^{2}, \ldots a_{i}^{k}\right)
$$

where $x_{i}$ is the $\mathrm{i}^{\text {th }}$ approach, $k$ is the number of amplitude values per approach and $a_{i}^{\circ}$ describes the first amplitude value of the $\mathrm{i}^{\text {th }}$ approach. For the example we are using, we will set the number of approaches to $i=n$. Writing this in matrix form looks as follows:

$$
X=\left(\begin{array}{cccc}
a_{1}^{1} & a_{1}^{2} & \ldots & a_{1}^{k} \\
\hline a_{2}^{1} & a_{2}^{2} & \ldots & a_{2}^{k} \\
\hline & \vdots & & \\
\hline a_{n}^{1} & a_{n}^{2} & \ldots & a_{n}^{k}
\end{array}\right)=\left(\begin{array}{c}
x_{1}^{T} \\
x_{2}^{T} \\
\vdots \\
x_{n}^{T}
\end{array}\right)
$$

where $X \in \mathbb{R}^{n \times k}$. For later convenience, the mean is subtracted using:

$$
\mu=\frac{1}{n} \sum_{i=1}^{n} x_{i}=\left(\frac{1}{n} \sum_{i=1}^{n} x_{i 1}, \ldots, \frac{1}{n} \sum_{i=1}^{n} x_{i d}\right)^{T}
$$

Next, the covariance is calculated using:

$$
C=\frac{1}{n-1} \sum_{i=1}^{n}\left(x_{i}-\mu\right)\left(x_{i}-\mu\right)^{T}=\frac{1}{n-1} X^{T} X
$$

The covariance matrix $C$ describes the covariance between the $i$-th and $k$-th elements on the $i, k$ position in the matrix. The covariance describes how likely variables correspond, a measure of how much the two coordinates vary together. The sign of the covariance shows the tendency in the linear relationship between the variables. The 
magnitude of the covariance is not easy to interpret, because it depends on the magnitude of the variables.

The covariance matrix $C$ is symmetric, thus can be diagonalized to $C=V L V^{T}$, where $V$ is the matrix with the columns composed of the eigenvectors and $L$ a diagonal matrix with $\lambda_{i}$ as the eigenvalues. The eigenvectors are the principal axes of the data. Projections of the data on the principle axes are called the principle components.

The data matrix $X$ can be decomposed using singular value decomposition, which yields $X=U S V^{T}$. $U$ is a unitary matrix and $S$ a diagonal matrix with singular values $s_{i}$. Filling $X=U S V^{T}$ into $(n-1) C=X^{T} X$ yields:

$$
(n-1) C=\left[U S V^{T}\right]^{T}\left[U S V^{T}\right]=V S^{T} U^{T} U S V^{T}=V S^{2} V^{T}=V L V^{T}
$$

This means that the singular vectors $V$ are the principal axes and its singular values are related to the eigenvalues from $C$ via $\lambda_{i}=s_{i}^{2}(n-1)$. The principal components are given by $X V=U S V^{T}=U S$.

To conclude, it is important to understand that PCA is not a smoothing filter. The variance represents the importance of a component. PCA finds the variance of every component. If a component contains signal information, and the signal is present in many data points, it will have a large variance. A component that only contains noise, however, will have a low variance, because noise is random and cannot add up. THerefore, deleting the low variance component will increase the SNR without losing information. For a more detailed description of PCA or SVD please have a look at these references $[58-60]$.

\subsubsection{PCA applied to AFM data}

The experiments shown in this chapter use the first 7 principle components. This number preserves the information in the data, but removes the noise. Using fewer principle components (less than 5) results in smoother data where important features of the data have been lost. Using more principle components (more than 10) results in more noise, but not an increase in features in the data. This number was determined empirically, by examining a force map of several different measurements. An example of the power of the PCA routine is shown in figure 2.13. The raw data 

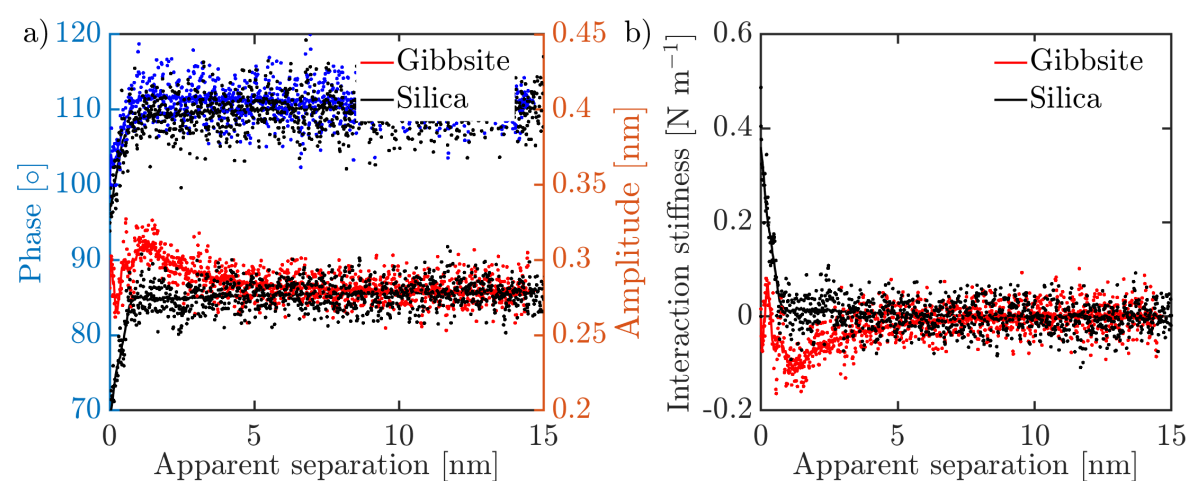

Figure 2.13: a) Amplitude (top curves) and phase (lower curves) versus distance recorded with AM AFM with a cantilever tip on silica (black) and gibbsite (upper blue curve and lower red curve). The dots are measurement data, and the lines are the PCA corrected data. b) The amplitude and phase data in a) converted to an interaction stiffness versus distance curve. The black and red curve is the interaction force on silica and gibbsite.

(dots) shows a high level of noise, whereas the PCA corrected data mostly removed the noise, meanwhile retaining the shape and details of the data. This noise depends on the experiment, but it is typically smaller than shown in figure 2.13. The effect of the PCA routine on the data is significant. The forces presented here are of low magnitude and close to the resolution of what we can measure with amplitude modulation AFM. This means that the signal to noise ratio is low, and therefore by using PCA we can restore some features in the data that was obscured by noise. Converting the amplitude and phase data, like shown in figure $2.13 \mathrm{a}$, to the interaction stiffness results in the data shown in figure $2.13 \mathrm{~b}$. As is clear from the graph, after converting the data to the interaction force, the details and shape of the data is preserved. The effect of the PCA routine is not only noticeable in the force distance curves, but improves the clarity in the force maps as well, as is visible in figure 2.14. The untouched data has noise that is close to the magnitude of the signal, obscuring the features of the data. Using the PCA routine removes this noise, clearing up the image, revealing features like the particle boundary. Averaging would also help to reduce the noise in the data, but this sacrifices lateral resolution. In chapter 6 the PCA routine will be used in order to increase the signal to noise ratio, since the measured forces are very weak. 

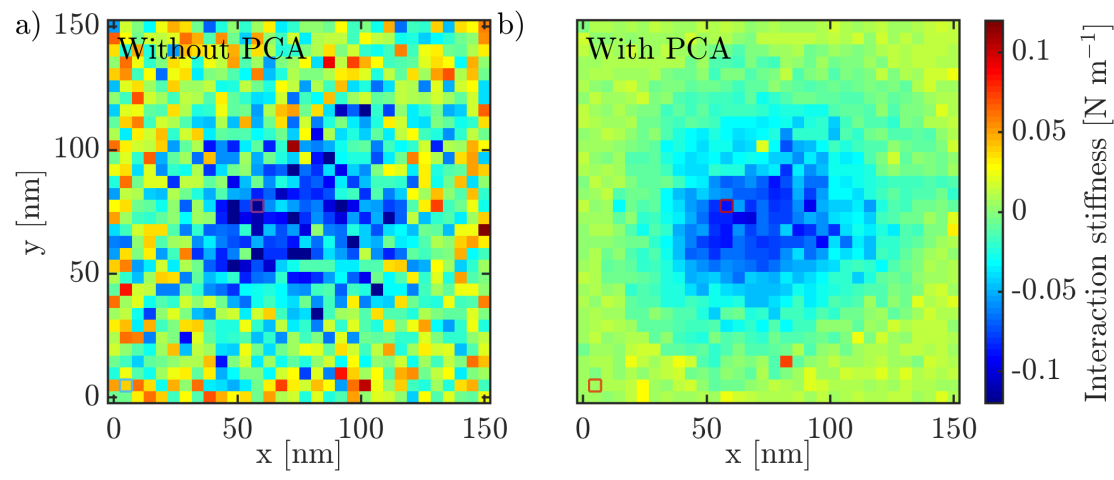

Figure 2.14: Interaction stiffness maps of gibbsite on silica in a $5 \mathrm{mM} \mathrm{CaCl}$ solution at $2 \mathrm{~nm}$ from the surface. The colors indicate the magnitude of the interaction stiffness, where red is a repulsive interaction and blue an attractive interaction. The marked squares are the forces shown in figure 2.13. In b) the PCA analyzed interaction stiffness map.
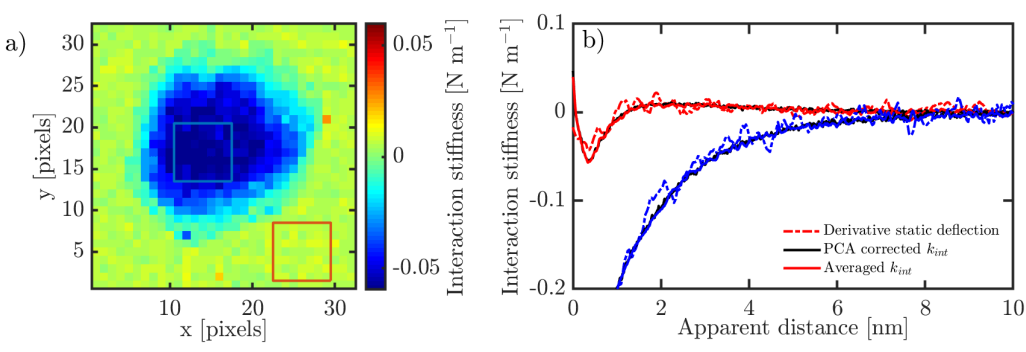

Figure 2.15: a) Interaction stiffness map of gibbsite on silica in a $10 \mathrm{mM} \mathrm{CaCl}$ solution at $3.0 \mathrm{~nm}$ from the surface. The colors indicate the magnitude of the interaction stiffness, where red is a repulsive interaction and blue an attractive interaction. The marked squares are the forces shown in the graph on the right. b) The solid colored lines are the average of the approaches on silica and gibbsite from a), shown in red and blue, respectively. In black the PCA cleaned data. The dashed colored lines are the derivative of the static deflection, which can serve as an additional check for data quality. 


\section{Bibliography}

[1] R. L. Bates, J. A. Jackson, and M. Gary, Glossary of geology. Falls Church, Va.: American Geological Institute, 2 d ed., 1980.

[2] W. A. Deer, R. A. Howie, and J. Zussman, An introduction to the rock-forming minerals. Harlow, Essex, England New York, NY: Longman Scientific \& Technical ; Wiley, 2nd ed., 1992.

[3] S. Guggenheim and R. T. Martin, "Definition of clay and clay mineral - joint report of the aipea nomenclature and cms nomenclature committees," Clays and Clay Minerals, vol. 43, no. 2, pp. 255-256, 1995.

[4] W. A. Deer, R. A. Howie, J. Zussman, and J. F. W. Bowles, "Rock-forming minerals," 2003.

[5] G. Brown, "Crystal-structures of clay-minerals and related phyllosilicates," Philosophical Transactions of the Royal Society a-Mathematical Physical and Engineering Sciences, vol. 31 , no. 1517, pp. 221-240, 1984.

[6] S. H. Sutheimer, P. A. Maurice, and Q. H. Zhou, "Dissolution of well and poorly crystallized kaolinites: Al speciation and effects of surface characteristics," American Mineralogist, vol. 84, no. 4, pp. 620-628, 1999.

[7] E. Tombacz and M. Szekeres, "Surface charge heterogeneity of kaolinite in aqueous suspension in comparison with montmorillonite," Applied Clay Science, vol. 34, no. 1-4, pp. 105-124, 2006.

[8] M. D. A. Bolland, A. M. Posner, and J. P. Quirk, "Ph-independent and phdependent surface-charges on kaolinite," Clays and Clay Minerals, vol. 28, no. 6, pp. $412-418,1980$.

[9] V. Gupta and J. D. Miller, "Surface force measurements at the basal planes of ordered kaolinite particles," Journal of Colloid and Interface Science, vol. 344, no. 2, pp. $362-371,2010$.

[10] J. Lutzenkirchen, A. Abdelmonem, R. Weerasooriya, F. Heberling, V. Metz, and R. Marsac, "Adsorption of dissolved aluminum on sapphire-c and kaoli- 
nite: implications for points of zero charge of clay minerals," Geochem Trans, vol. 15, p. 9, 2014.

[1 1 ] J. Liu, L. Sandaklie-Nikolova, X. M. Wang, and J. D. Miller, "Surface force measurements at kaolinite edge surfaces using atomic force microscopy," Journal of Colloid and Interface Science, vol. 420, pp. 35-40, 2014.

[12] A. Klaassen, F. Liu, D. van den Ende, F. Mugele, and I. Siretanu, "Impact of surface defects on the surface charge of gibbsite nanoparticles," Nanoscale, 2017.

[13] G. Sposito, The Environmental Chemistry of Aluminum, Second Edition. Taylor and Francis, 1995.

[14] B. R. Bickmore, C. J. Tadanier, K. M. Rosso, W. D. Monn, and D. L. Eggett, "Bond-valence methods for pk(a) prediction: critical reanalysis and a new approach," Geochimica Et Cosmochimica Acta, vol. 68, no. 9, pp. 2025-2042, 2004.

[15] A. Vyalikh, K. Zesewitz, and U. Scheler, "Hydrogen bonds and local symmetry in the crystal structure of gibbsite," Magnetic Resonance in Chemistry, vol. 48, no. 11, pp. $877-881,2010$.

[16] A. Michalkova, D. Tunega, and L. T. Nagy, "Theoretical study of interactions of dickite and kaolinite with small organic molecules," Journal of Molecular Structure-Theochem, vol. 581, pp. 37-49, 2002.

[17] D. Tunega, M. H. Gerzabek, and H. Lischka, "Ab initio molecular dynamics study of a monomolecular water layer on octahedral and tetrahedral kaolinite surfaces," Journal of Physical Chemistry B, vol. 108, no. 19, pp. 5930-5936, 2004.

[18] I. Siretanu, D. Ebeling, M. P. Andersson, S. L. Stipp, A. Philipse, M. C. Stuart, D. van den Ende, and F. Mugele, "Direct observation of ionic structure at solidliquid interfaces: a deep look into the stern layer," Sci Rep, vol. 4, p. 4956, 2014.

[19] T. Hiemstra and W. H. Vanriemsdijk, "Physical-chemical interpretation of primary charging behavior of metal (hydr)oxides," Colloids and Surfaces, vol. 59, pp. 7-25, 1991.

[20] L. Parfitt R, R. Fraser A, D. Russell J, and C. Farmer V, "Adsorption on hydrous oxides: Ii. oxalate, benzoate and phosphate on gibbsite," Journal of Soil Science, vol. 28 , no. 1 , pp. 40-47, 1977. 
[21] R. Kummert and W. Stumm, "The surface complexation of organic acids on hydrous $\gamma$-al203," Journal of Colloid and Interface Science, vol. 75, no. 2, pp. 373385,1980 .

[22] D. E. Yates and T. W. Healy, "The structure of the silica/electrolyte interface," Journal of Colloid and Interface Science, vol. 55, no. 1, pp. 9-19, 1976.

[23] J.E. Sader, "Frequency response of cantilever beams immersed in viscous fluids with applications to the atomic force microscope," Journal of Applied Physics, vol. 84 , no. 1 , pp. $64-76,1998$.

[24] J. E. Sader, J. W. M. Chon, and P. Mulvaney, "Calibration of rectangular atomic force microscope cantilevers," Review of Scientific Instruments, vol. 70, no. 10, pp. 3967-3969, 1999.

[25] H. J. Butt and M. Jaschke, "Calculation of thermal noise in atomic-force microscopy," Nanotechnology, vol. 6, no. 1, pp. 1-7, 1995.

[26] A. Labuda, J. Cleveland, N. A. Geisse, M. Kocun, B. Ohler, R. Proksch, M. B. Viani, and D. Walters, "Photothermal excitation for improved cantilever drive performance in tapping mode atomic force microscopy," Microscopy and Analysis28, vol. 3, pp. S21-S25, 2014.

[27] H. Hölscher, B. Gotsmann, and A. Schirmeisen, "Dynamic force spectroscopy using the frequency modulation technique with constant excitation," Physical Review B, vol. 68, no. 15, p. 153401, 2003.

[28] T. R. Albrecht, P. Grutter, D. Horne, and D. Rugar, "Frequency-modulation detection using high-q cantilevers for enhanced force microscope sensitivity," Journal of Applied Physics, vol. 69, no. 2, pp. 668-673, 1991.

[29] H. Holscher, B. Gotsmann, W. Allers, U. D. Schwarz, H. Fuchs, and R. Wiesendanger, "Measurement of conservative and dissipative tip-sample interaction forces with a dynamic force microscope using the frequency modulation technique," Physical Review B, vol. 64, no. 7, 2001.

[30] R. Garcia and R. Perez, "Dynamic atomic force microscopy methods," Surface Science Reports, vol. 47, no. 6-8, pp. 197-301, 2002. 
[31] L. Landau and E. Lifshitz, Fluid Mechanics: Volume 6. Elsevier Science, 1987.

[32] R. Iler and I. R. K, The Chemistry of Silica: Solubility, Polymerization, Colloid and Surface Properties and Biochemistry of Silica. Wiley, 1979.

[33] B. Derjaguin and L. Landau, "The theory of stability of highly charged lyophobic sols and coalescence of highly charged particles in electrolyte solutions," Acta Physicochim. URSS, vol. 14, no. 633-662, p. 58, 1941.

[34] E. J. W. Verwey, “Theory of the stability of lyophobic colloids," The Journal of Physical and Colloid Chemistry, vol. 51, no. 3, pp. 631-636, 1947.

[35] J. Lyklema, Fundamentals of interface and colloid science. San Diego: Academic Press, 2000.

[36] S. J. O'Shea and M. E. Welland, "Atomic force microscopy at solid-liquid interfaces," Langmuir, vol. 14, no. 15, pp. 4186-4197, 1998.

[37] P. Leroy and A. Revil, "A triple-layer model of the surface electrochemical properties of clay minerals," Journal of Colloid and Interface Science, vol. 270, no. 2, pp. $371-380,2004$.

[38] C. Tournassat, Y. Chapron, P. Leroy, M. Bizi, and F. Boulahya, "Comparison of molecular dynamics simulations with triple layer and modified gouy-chapman models in a 0.1 m nacl-montmorillonite system," Journal of Colloid and Interface Science, vol. 339, no. 2, pp. 533-541, 2009.

[39] R.J. Hunter, Zeta potential in colloid science : principles and applications. Colloid science, London ; New York: Academic Press, 1981.

[40] H.-J. Butt, K. Graf, and M. Kappl, Physics and chemistry of interfaces. Physics textbook, Weinheim: Wiley-VCH, 2003.

[41] J. Lyklema, "Quest for ion-ion correlations in electric double layers and overcharging phenomena," Advances in Colloid and Interface Science, vol. 147-48, pp. 205-213, 2009.

[42] R. Kjellander, "Intricate coupling between ion-ion and ion-surface correlations in double layers as illustrated by charge inversion-combined effects of strong 
coulomb correlations and excluded volume," J Phys Condens Matter, vol. 21, no. 42, p. $424101,2009$.

[43] M. E. Fleharty, F. van Swol, and D. N. Petsev, "Solvent role in the formation of electric double layers with surface charge regulation: A bystander or a key participant?," Phys Rev Lett, vol. 116, no. 4, p. 048301, 2016.

[44] T. A. Gmür, A. Goel, and M. A. Brown, "Quantifying specific ion effects on the surface potential and charge density at silica nanoparticle-aqueous electrolyte interfaces," The Journal of Physical Chemistry C, vol. 120, no. 30, pp. 1661716625, 2016.

[45] A. A. Hassanali and S. J. Singer, "Model for the water-amorphous silica interface: The undissociated surface," Journal of Physical Chemistry B, vol. 111, no. 38, pp. $11181-11193,2007$.

[46] A. A. Hassanali, H. Zhang, C. Knight, Y. K. Shin, and S. J. Singer, "The dissociated amorphous silica surface: Model development and evaluation," Journal of Chemical Theory and Computation, vol. 6, no. 11, pp. 3456-3471, 2010.

[47] G. Trefalt, S. H. Behrens, and M. Borkovec, "Charge regulation in the electrical double layer: Ion adsorption and surface interactions," Langmuir, 2015.

[48] B. W. Ninham and Parsegia.Va, "Electrostatic potential between sufraces bearing ionizable groups in ionic equilibrium with physiologic saline solution," Journal of Theoretical Biology, vol. 31, no. 3, p. 405, 1971.

[49] S. L. Carnie and D. Y. C. Chan, "Interaction free-energy between plates with charge regulation - a linearized model," Journal of Colloid and Interface Science, vol. 161 , no. 1 , pp. 260-264, 1993.

[50] T. Markovich, D. Andelman, and R. Podgornik, "Charge regulation: A generalized boundary condition?," EPL (Europhysics Letters), vol. 113, no. 2, p. 26004, 2016.

[51] J. N. Israelachvili, "Measurement of forces between surfaces immersed in electrolyte-solutions," Faraday Discussions, vol. 65, pp. 20-24, 1978. 
[52] C. L. Zhao, D. Ebeling, I. Siretanu, D. van den Ende, and F. Mugele, "Extracting local surface charges and charge regulation behavior from atomic force microscopy measurements at heterogeneous solid-electrolyte interfaces," Nanoscale, vol. 7, no. 39, pp. 16298-16311, 2015.

[53] D. Y. C. Chan and D. J. Mitchell, “The free-energy of an electrical double-layer," Journal of Colloid and Interface Science, vol. 95, no. 1, pp. 193-197, 1983.

[54] S. H. Behrens and M. Borkovec, "Electric double layer interaction of ionizable surfaces: Charge regulation for arbitrary potentials," Journal of Chemical Physics, vol. 111, no. 1, pp. 382-385, 1999.

[55] S. H. Behrens and D. G. Grier, "The charge of glass and silica surfaces," Journal of Chemical Physics, vol. 115, no. 14, pp. 6716-6721, 2001.

[56] S. H. Behrens and M. Borkovec, "Electric double layer interaction of ionizable surfaces: Charge regulation for arbitrary potentials," Journal of Chemical Physics, vol. 111 , no. 1, pp. 382-385, 1999.

[57] T. Hiemstra, W. Van Riemsdijk, and G. Bolt, "Multisite proton adsorption modeling at the solid/solution interface of (hydr) oxides: A new approach: I. model description and evaluation of intrinsic reaction constants," Journal of colloid and interface science, vol. 133, no. 1, pp. 91-104, 1989.

[58] “Singular-value decomposition," 2018.

[59] "Principle component analysis," 17-07-2018 2018.

[6o] A. Perunicic, "How are principal component analysis and singular value decomposition related?” 2017. 
"It's coarse and rough and irritating and it gets everywhere."

Anakin

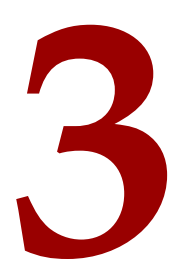

\section{Ionic strength and $\mathrm{pH}$ dependence of the diffuse layer charge of Silica-electrolyte interfaces, measured with AFM}

We USE High Resolution Atomic Force Microscopy to probe the surface chemistry behavior of silica surface in aqueous solutions of variable $\mathrm{pH}$ and $\mathrm{NaCl}$ concentration. We are able to extract diffuse layer charge values from the experimental force-distance curves within $1 \%$ accuracy using an exponential surface charge (ESC) - potential relation. Using the ESC model enables the separation of diffuse layer charge/potential calculations from surface chemistry. A 2 pK model that describes the silanol deprotonation equilibrium constants depends on the $\mathrm{pH}$ of the electrolyte solution and tip geometry. This indicates that a single $\mathrm{pK}$ pair cannot describe the diffuse layer charge chemistry of silica substrates adequately. Linking the charge values to the relative occupation of surface sites is not reliable, because the chemical modeling of the substrate still fails. However, colloidal probe force spec- 
troscopy and diffuse layer charge fitting with exponential surface charge modeling is still a valuable method to determine the diffuse layer charge. The obtained diffuse layer charge values can be used in further chemical modeling of the surface or for quantitative comparisons with other methods.

\subsection{Introduction}

When a solid is immersed in an aqueous liquid, it generally acquires a surface charge through adsorption or desorption of ionic surface groups. These surface reactions play an important role in many chemical, physical, biological and technological processes ${ }^{[1-4]}$. To understand the mechanisms behind these processes, an essential step is to study the charging properties of electrolyte-solid interfaces and their dependence on the electrolyte composition $[5-[16]$. The charge in and near the solidelectrolyte interface originates from the equilibrium between the (de-) protonation of surface groups and the ad- and desorption of ions $[17-19]$. The region where the charge from (de-) protonation reactions is balanced by ions in the liquid, is called the electric double layer (EDL). The electric double layer generally consists of an adsorbed layer of ions, called Stern layer, and a region of electrically attracted and repelled ions that are free to move under the influence of hydrodynamic and thermal effects, which is called the diffuse layer [20,21].

Different techniques are used to measure the various types of charge. Recently, $\mathrm{x}$-ray reflectivity techniques have enabled a detailed look into the ion distribution near the solid-liquid interface, giving a clear picture of the Stern layer [5, 22]. X-ray techniques however, require atoms with a significant large charge density for detection. This complicates studying the more common ions such as sodium and chloride. With molecular dynamics (MD) simulations the structure and dynamics of the EDL can be explored too, which helps to improve models and the interpretation of experiments [23]. MD simulations can mostly resolve molecular-level mechanisms only, because a large system size requires more computing power than currently available. Titration measurements can determine the intrinsic surface charge of oxide surfaces. It offers good statistics due to the averaging over many particles [21,24-26]. However, in contrast to the intrinsic surface charge, there is no direct way to quantify the diffuse layer charge. Electrokinetic techniques, such as streaming potential measure- 
ments and electrophoresis can quantify the diffuse layer charge. Despite the simplicity of these techniques, the interpretation of the experimental data remains difficult due to the need of elaborate models. In these models, properties such as the location of the shear plane or the hydrodynamic boundary conditions are difficult to determine $[27,10,28-[31]$. Direct force measurements, such as SFA and atomic force microscopy (AFM) are a reliable way of measuring the diffuse layer charge. The advantage of SFA is that it has an excellent distance resolution of around $1 \AA[32, \sqrt{33}]$. However, it is mostly restricted to mica as a substrate, because the technique asks for large atomically smooth surfaces. Using AFM with a colloidal probe, the forces between tip and substrate can be measured for a wider range of substrates. For a correct interpretation of the diffuse layer charge obtained from direct force measurements, it is essential to implement the charge regulation correctly [34-[36] (see chapter 2).

A problem common to all these measurement techniques, is the spread in reported surface potentials and charge values. The reported point of zero charge (PZC) values for clay minerals such as kaolinite, gibbsite and montmorillonite can vary several $\mathrm{pH}$ units [37,11]. Even for simple systems such as hydroxide surfaces, a large spread in the values for the PZC and equilibrium constants of the surface reactions can be found. This can be explained to some extent by the heterogeneity of particles, where particles with facets that have different PZC can lead to a variation in the overall PZC. Also differences in the surface site density of nominally equal materials can lead to an overall difference in the PZC. However, Szekeres et al. argue that the scatter in the range of several $\mathrm{pH}$ units in the reported $\mathrm{PZC}$ values cannot be explained by deviations in the crystal structure or surface energy only [38].

Even for a well studied system as the silica-electrolyte interface many uncertainties remain. For titration, the experimental results for the charging characteristics are reasonably consistent and the intrinsic surface charge can often be approximated by a 2pK surface complexation model (SCM) [19] or a $1 \mathrm{pK}$ Basic Stern model [39]. However, for electrokinetic and direct force measurements, from which the diffuse layer charge can be determined, the results are less clear. Often the standard electric double layer models, like the TLM, BSM and GCM are able to describe the measurements sufficiently over either a wide range of salt concentration, or a wide range of $\mathrm{pH}$ values. Whether the $2 \mathrm{pK}$ values are able to describe the diffuse layer charge for a wide range of salt concentrations and $\mathrm{pH}$ values remains unclear. Moreover, sys- 
tematic studies where both the salt concentration and the $\mathrm{pH}$ are varied in the same experiment, are missing.

In this work we use colloidal probe force spectroscopy to measure the interaction forces between a silica substrate and a silica probe in various sodium chloride solutions within a wide range of $\mathrm{pH}$ values. In addition, we accurately determine the zero-point on the tip-sample distance scale, which is paramount in atomic force microscopy. To check for consistency we also compare the dynamic force spectroscopy results with the static mean deflection results. Subsequently, the diffuse layer charge density has been calculated using DLVO theory, charge regulation and the differential surface capacitance model mentioned in chapter 2 . These diffuse layer charge data have been used to test earlier proposed surface reaction schemes for the silica substrates. From our results we conclude that it is impossible to model the surface charge behavior on silica substrates accurately with a regular single site $2 \mathrm{pK}$ model using a single pair of fixed $\mathrm{pK}$ values for a wide range of $\mathrm{pH}$ values and salt concentrations.

\subsection{Methods}

\subsubsection{Substrate and probe preparation}

Silicon substrates with a $30 \mathrm{~nm}$ thick thermally grown oxide layer were used as a substrate. The silica is cleaned in an ultrasonic bath, first for 15 minutes in a solution of isopropanol, ethanol and Millipore water (25/25/50\% by volume) and subsequently another 15 minutes in Millipore water only. Next, the substrate is plasma cleaned (PDC-32G-2, Harrick Plasma) for 20 minutes. Sodium chloride (puriss, ACS reagent grade, Sigma Aldrich) solutions are prepared by dissolving salt in Millipore water. The $\mathrm{pH}$ is adjusted by adding $\mathrm{HCl}$ or $\mathrm{NaOH}$ solutions. All experiments are performed under liquid, in a closed fluid cell that allows for liquid exchange and is kept at a constant temperature of $29.7^{\circ} \mathrm{C}$. The fluid is exchanged using two syringes by injecting a new solution while removing the old solution. During fluid exchange about 25 times the total sample volume in the fluid cell is exchanged. To equilibrate the system a next measurement is started only ten minutes after finishing the fluid exchange. We use silicon colloidal probes (Team NanoTec LRCH 750/Cr-Au) cov- 


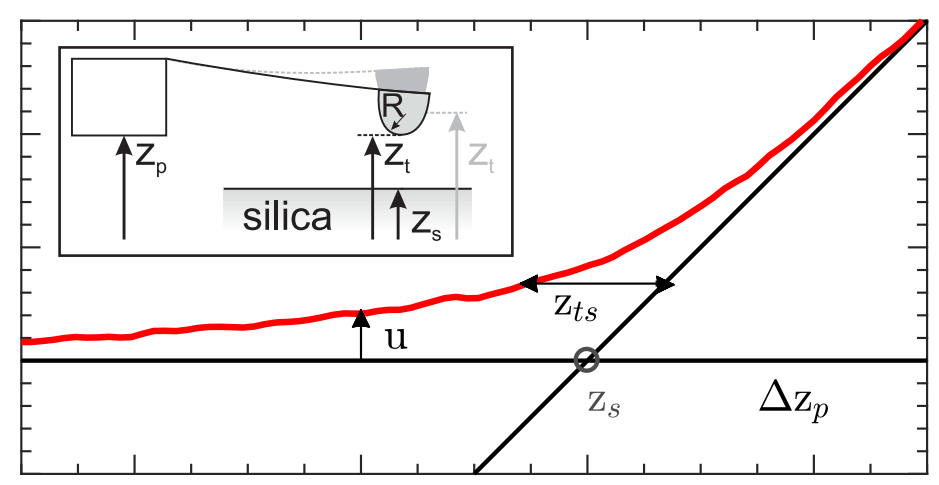

Figure 3.1: Schematic of silica-silica colloidal probe measurements. In red the deflection $u$ as a function of the piezo displacement. The definitions of the piezo distance $z_{p}$, the sample distance $z_{s}$, the tip distance $z_{t}$, the deflection $u=z_{t}-z_{s}$, the tip-sample distance $z_{t s}=z_{p}-z_{t}$, and tip radius $R$ are defined as depicted.

ered by a 1-2 nm thick native oxide layer and a golden backside coating on the cantilever. Before use, the cantilevers are cleaned using a mixture of isopropanol and ethanol (50/50\% by volume) and after drying, they are plasma cleaned for 15-20 minutes.

\subsubsection{AFM force spectroscopy}

Dynamic force spectroscopy measurements were performed with a commercial Asylum Research Cypher ES equipped with photothermal excitation. A schematic representation of the set-up can be found in figure 3.1 .

The AFM is used in amplitude modulation (AM) with force ramp mode. The force ramp mode records the mean deflection $u$ (figure $3.2 \mathrm{a}$ ), amplitude $A$ (figure $3.2 \mathrm{~b}$ ), phase $\varphi(3.2 \mathrm{c})$, drive frequency $\left(\omega_{d}\right)$ and the measured piezo position $\left(z_{p p}\right)$ of the cantilever as function of the piezo distance $\left(z_{p}\right)$. The approach and retraction speed of the cantilever is set to $150 \mathrm{~nm} \mathrm{~s}^{-1}$. The set point for reversing the cantilever motion from approaching to retracting is based on the amplitude $A$ or on the mean deflection $u$. Using the amplitude as a set point, the direction is reversed when $A$ is reduced 
to $10 \%$ of the free amplitude $A_{\infty}$. We consider this amplitude criterion to result in a non-contact (nc) method. In case the mean deflection is used, the direction is reversed when $u$ has reached a critical value chosen in the range of $10-20 \mathrm{~nm}$ (figure 3.2a). Typically, around 30 approaches for each fluid composition are recorded using this set point. When the mean deflection criterion is used, the cantilever tip comes in hard-contact with the substrate. In this case the number of approaches is reduced to 5-10, to prevent damage to the tip. These 'hard-contact' (hc)force-distance curves are used to calibrate the tip-sample distance.

At the beginning of the experiment, the system is calibrated, in liquid, to get the correct amplitude sensitivity and spring constant. The driving force to excite the cantilever is chosen to guarantee a small amplitude $(<1 \mathrm{~nm})$ of the freely oscillating cantilever. Next, force distance curves are recorded for each fluid composition using both non- and hard-contact mode. After recording the force-distance curves for each specific condition, the power spectral density is recorded at a tip-substrate distance of $300 \mathrm{~nm}$, where the tip-sample interaction is assumed to be negligible, such that the correct resonance frequency $\left(\omega_{r}\right)$ and quality factor $(Q)$ can be used in the analysis. After having collected the data for all fluid compositions (different $\mathrm{pH}$ and $\mathrm{NaCl}$ concentrations), the measurements at the initial fluid composition are repeated to verify whether any permanent changes, like change in tip radius or substrate damage have occurred during the experiment. After this check, the spring constant is calibrated once more, to verify that the stiffness has not changed during the experiment.

\subsubsection{Data handling}

During experiments, thermal drift causes a slight offset in the real z piezo position and so in the deflection, amplitude and phase versus piezo position curves. Therefore, offset correction of the individual approach curves is the first step in the data analysis. This is achieved by calculating the cross correlation between the individual curves (after omitting the first 3 approaches), using the amplitude channel. The latter typically shows most pronounced features, making the alignment most accurate. For a range of measured $A_{n}$-values, say $N$ points around half the maximum amplitude value $A_{\infty}$, the difference $\delta_{n}=z_{n}-z_{n}^{(1)}$ in piezo position $z_{n}$ between both curves at that $A_{n}$ value is calculated. Here the value $A_{n}^{(1)}$ of the reference curve is obtained by interpolation between neighboring $A$ values. The average $\langle\delta\rangle=N^{-1} \sum_{n=1}^{N} \delta_{n}$ is considered 

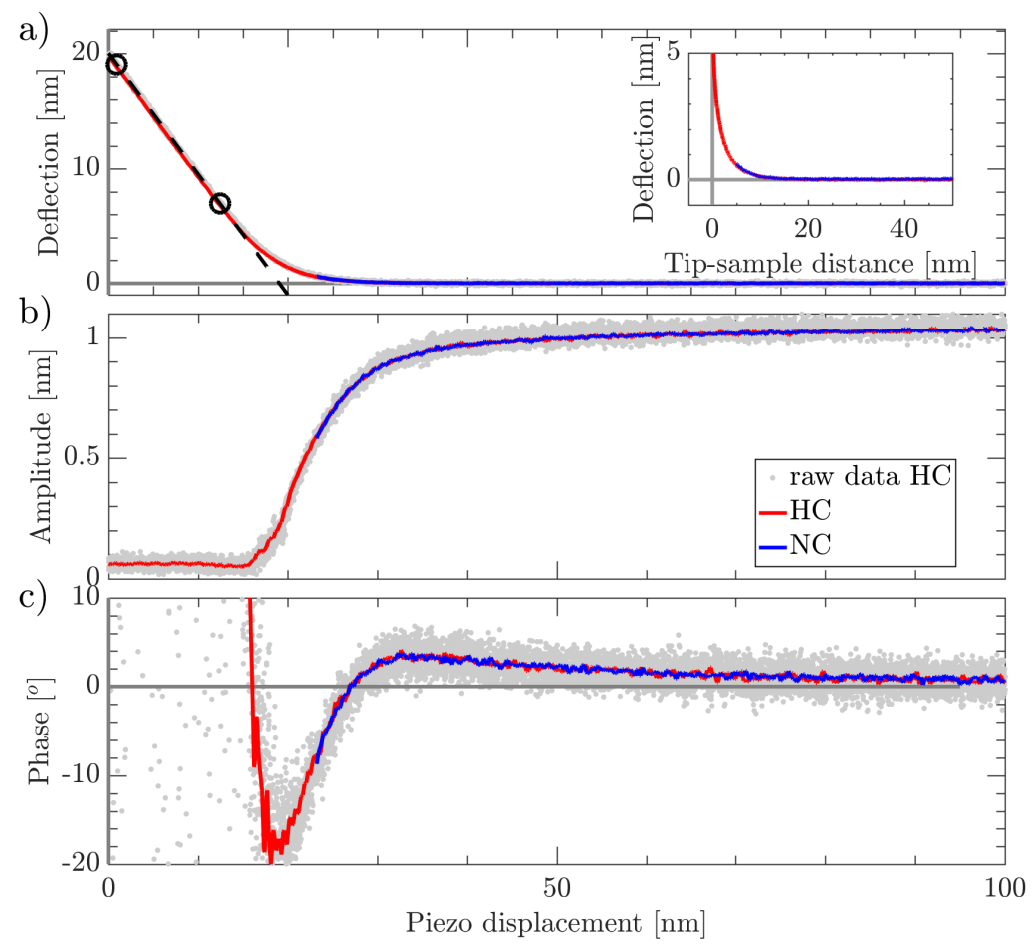

Figure 3.2: Examples of a) the cantilever deflection, b) amplitude and c) phase versus piezo position in a $10 \mathrm{mM} \mathrm{NaCl} \mathrm{pH} 9$ solution. The inset in a) shows the conversion to the 'deflection versus tip-sample distance' curve. $\left(k_{c}=0.96 \mathrm{~N} \mathrm{~m}^{-1}, \omega_{d}=27.0 \mathrm{kHz}\right.$, $\omega_{\mathrm{o}} / 2 \pi=29.2 \mathrm{kHz}, R \approx 750 \mathrm{~nm}$ and $Q=2.3$ ) 
to be the offset between both approach curves; it is subtracted from all $z_{n}$ values of the considered curve. On average the offset between the approach curves for the 'non-contact' method is about $1 \mathrm{~nm}$, and for the 'hard-contact' method about $0.5 \mathrm{~nm}$. In this way two master curves are generated which contain all data points, one for the non-contact $(\mathrm{NC})$ curves, and one for hard-contact $(\mathrm{HC})$ curves. To reduce the noise, the averaged curves are smoothed by binning the distance scale (bin-size of $0.1 \mathrm{~nm}$ ) and fitting a straight line through all data points within a bin. From this fit the value at the center of the bin has been determined. Next, the NC master curve is aligned to the $\mathrm{HC}$ curve using once more the method just described. These master curves are used to obtain the final force-distance curves, following the procedure described by Liu et al. [40,41], which we shortly describe for completeness.

The result of a measurement in a $10 \mathrm{mM} \mathrm{NaCl} \mathrm{pH} 9$ solution is shown in Figure 3.2. The red and blue curves are the averaged curves of the data recorded using hardcontact ( $\mathrm{HC})$ and non-contact (NC), respectively. The curves are an average of 4 $\mathrm{HC}$ and $25 \mathrm{NC}$ approaches. The $\mathrm{HC}$ raw data are shown in light gray. The curves shown are already aligned using the procedure described above. In figure $3.2 \mathrm{a}$, the line, fitted to the linear part of the mean deflection curve, is shown in black. In this range, the tip is in hard-contact with the sample and the intersection of this line with the $u=\mathrm{o}$ axis $\left(z_{s}\right)$ is used for the calibration of the tip-sample distance. Despite the fact that the tip is in hard-contact with the substrate, the amplitude in figure 3.2 shows that the cantilever still has a finite amplitude. The data also show that the NC mode follows the HC mode very accurately.

\subsubsection{Determination of the tip-sample distance}

For a quantitative analysis of the force-distance curves, we have to convert the piezo position $z_{p}$ to the tip-sample distance $z_{t s}$ (figure 3.2/a inset). To this end we use the $\mathrm{HC}$ mean deflection $(u)$ curves. For deflections larger than $5 \mathrm{~nm}$, a straight line has been fitted to the measured $u$ versus $z_{p}$ curve, see figure 3.1 and $3.2 \mathrm{a}$. Extrapolating this line to zero deflection (the crossing with the z-axis), gives the position $\left(z_{s}\right)$ at which a non-deflected tip would touch the substrate. To obtain the real tip-sample distance, we have to add the cantilever deflection $u$ to the piezo position $z_{p}$ and cor- 
rect for $z_{s}$. So the tip-sample distance is given by:

$$
z_{t s}=z_{p}+u-z_{s}
$$

When the tip-sample distance approaches zero, the deflection increases rapidly. An upward deflection reflects a repulsive interaction, as expected for symmetric systems. At high salt concentrations (above $100 \mathrm{mM}$ monovalent salt) or low charge conditions (i.e. near the point of zero charge) an attractive interaction is observed, due to the van der Waals force, which for these conditions is stronger than the repulsive electrostatic interaction. Relying on the literature value for the Hamaker constant, we can estimate the tip radius $R$ from this van der Waals interaction.

\subsubsection{Force inversion}

Once we have established the tip-sample distance we convert the measured $A$ and $\varphi$ versus $z_{t s}$ curves into force-distance curves. We follow the force conversion for photothermal excitation procedure described in chapter 2. Here, we only give the force inversion equation (equation 2.9):

$$
k_{i n t}=k_{c}\left[1-\left(\frac{\omega}{\omega_{\circ}}\right)^{2}\right] \frac{A_{\infty} \cos \left(\varphi-\varphi_{\infty}\right)-A}{A}+\gamma_{c} \omega \frac{A_{\infty} \sin \left(\varphi-\varphi_{\infty}\right)}{A}
$$

The spring constant $k_{c}$, quality factor $Q$, resonance frequency $\omega_{0}$, effective mass $m^{*}=$ $k_{c} / \omega_{\mathrm{o}}^{2}$ and damping coefficient $\gamma_{c}=k_{c} /\left(\omega_{\mathrm{o}} Q\right)$ of the cantilever are determined during the experiment for each fluid composition, see section 2.2. Integrating the given equation, $F(z)=\int_{z}^{\infty} k_{\text {int }}\left(z^{\prime}\right) d z^{\prime}$, leads to the desired tip-sample interaction force. Except for the radius $R$ of the colloidal probe, which is determined using a scanning electron microscope, all cantilever properties in this work are determined under liquid. Typical values are $k_{c} \approx 3 \mathrm{~N} \mathrm{~m}^{-1}, \omega_{\mathrm{o}} / 2 \pi \approx{ }_{15} \mathrm{kHz}, R \approx 750 \mathrm{~nm}$ and $Q \approx 2.5$. When we take the derivative of the static deflection curve (static mode), we can compare it to the interaction stiffness $\left(k_{\text {int }}=-d F_{\text {int }} / d z\right)$ calculated using the force inversion equation (dynamic mode). This allows us to verify whether the results of the force inversion routine are in agreement with the static mode, as a quality check of our data. 


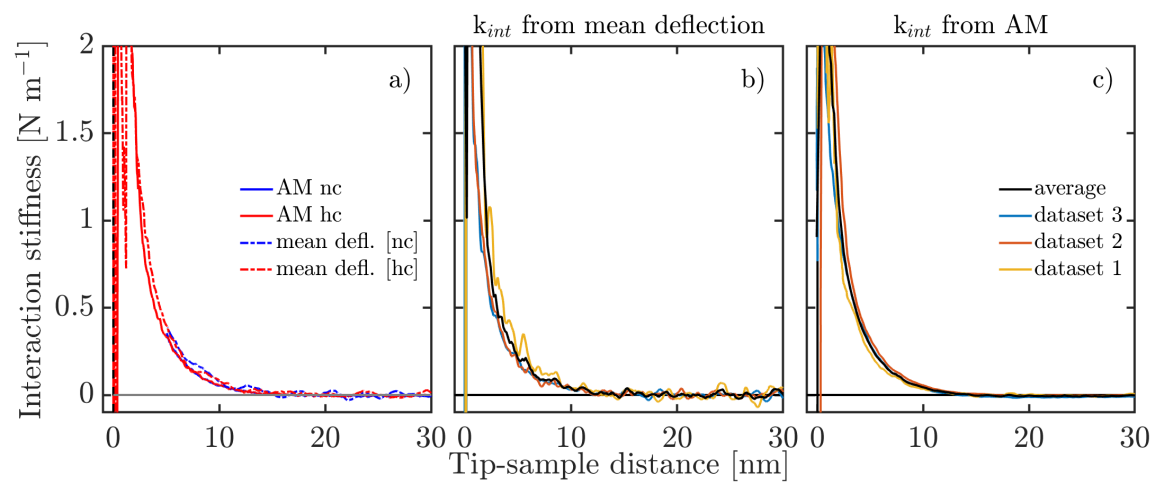

Figure 3.3: Interaction stiffness as function of tip-sample distance a) determined using the force inversion method (solid lines) and taking the derivative of the mean deflection (dashed lines) for the hard-contact (red) and non-contact (blue) method in a $10 \mathrm{mM}$ $\mathrm{NaCl} \mathrm{pH}$ g solution. b) The mean deflection curves for three separate measurements (red, blue and yellow) and its average (black). c) The amplitude modulation for three separate measurements (red, blue and yellow) and the average (black).

An example result of this comparison is shown in figure 3.3a. The full curves result from the force inversion (AM), whereas the dashed lines are (the numerical derivative of) the mean deflection curves. The AM and mean deflection curves match, as it should, since both measure the same interaction force. Despite averaging over many curves, the $k_{\text {int }}\left(z_{t s}\right)$ curves obtained from the mean deflection data are more noisy (figure $3.3 \mathrm{~b}$ shows data for three measurements). The small amount of noise in the mean deflection curves is significantly amplified by the numerical differentiation. When the AM (figure $3.3 \mathrm{c}$ shows data from three measurements) and mean deflection curves deviate significantly, that dataset is discarded in the analysis. The reason for a mismatch is not always clear, but happens mostly for low charge conditions (i.e. low concentration and low $\mathrm{pH}$ ). Possible causes could be a not well-equilibrated system or contamination.

The averaged AM curves obtained from three independent measurements recorded with three different cantilevers are used for the further analysis. The deviations between the three experiments is very small, which shows the great reproducibility, especially for tip-sample distances above $3 \mathrm{~nm}$. For most conditions the deviation of 
the individual curves from the averaged curve is less than $20 \%$. In general, best agreement is found at high charge conditions (i.e. high $\mathrm{pH}$ or salt concentrations), where the interactions are the strongest. However, below $2 \mathrm{~nm}$, large deviations between the different curves are observed.

Force-distance curves The accuracy of the diffuse layer charge fitting depends on several parameters. To quantify the error in the calculated diffuse layer charge due to uncertainty in the tip-sample distance, we take the force-distance curve for each fluid composition and shift the tip-sample distance by $\delta$ varying from -1 to $1 \mathrm{~nm}$ in steps of $0.1 \mathrm{~nm}$. Then we fit the diffuse layer charge and calculate the mean error for these shifts, resulting in the gradient $|d \sigma / d \delta|$. We estimate the inaccuracy in the tip-sample distance to be $\delta= \pm 0.5 \mathrm{~nm}$. This leads to an uncertainty $\sigma=|d \sigma / d \delta| \delta$ (see the error bars presented later on in figure 3.5). Furthermore, changing the lower fit boundary (around $5 \mathrm{~nm}$ ) by $2 \mathrm{~nm}$ results in a maximum of $15 \%$ change in the diffuse layer charge. Changing the upper fit boundary condition by $2 \mathrm{~nm}$ results in a maximum of $1 \%$ change in the diffuse layer charge. Other factors that can introduce inaccuracies in the diffuse layer charges are instrument sensitivity, errors in the calibration and errors introduced in the linearization for small amplitudes, which are minor compared to the errors estimated above.

\subsection{Results}

For the results we use the averaged AM curves obtained from three independent measurements as explained in section 2.5. To obtain insight in the concentration and $\mathrm{pH}$ dependence of the interactions, and ultimately in the diffuse layer charges, we plot the results on a linear-log scale, because the interactions for larger separations should scale as $k_{\text {int }} \sim e^{-\kappa z}$. In figure 3.4 the interaction stiffness versus the tip-sample distance has been shown for $\mathrm{pH}_{4}$ (panel a), $\mathrm{pH} 6$ (b) and $\mathrm{pH}_{9}$ (c) at various concentrations. From the linear tail, we can extract the Debye length $\kappa^{-1}$ and ionic strength $I_{\infty}$ using the equation $\kappa^{-1} \approx 0.304 / \sqrt{I_{\infty}}$, where $\kappa^{-1}$ is measured in $\mathrm{nm}$ and $I_{\infty}$ in mole $/ L^{[32]}$. In table 3.1 the extracted ion concentrations and the Debye lengths have been listed. The AM curves in figure 3.4 are an average of typically three experiments. An increasing salt concentration results in a steeper slope and so a shorter Debye length. Extrapolating the linear tail towards zero tip-sample distance 


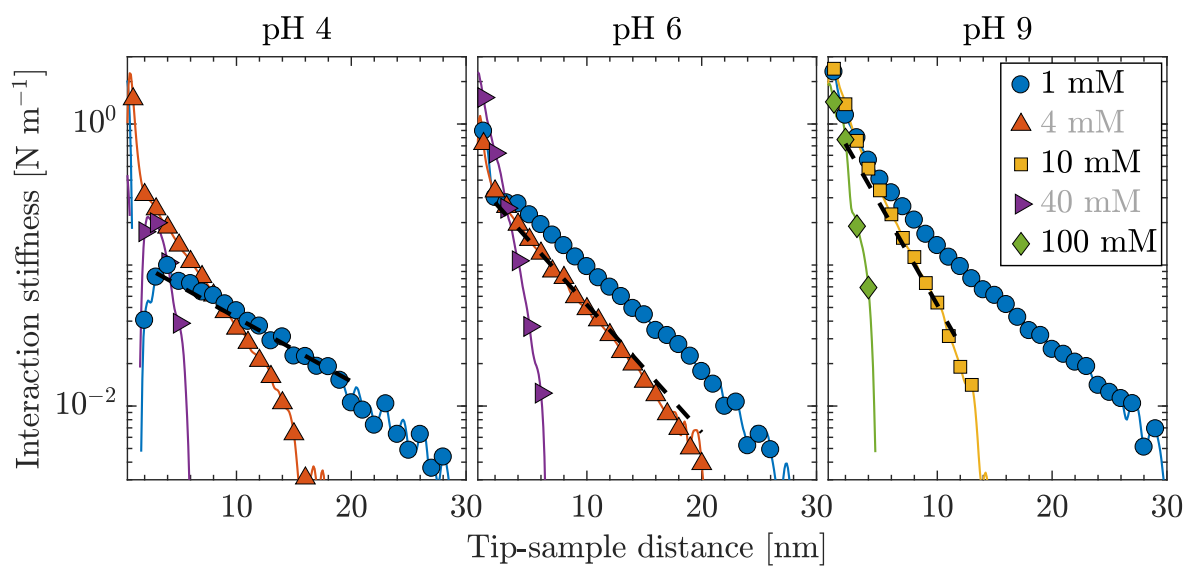

Figure 3.4: The interaction stiffness versus tip-sample distance for 1, 4, 10, 40 and $100 \mathrm{mM}$ $\mathrm{NaCl}$ solutions at $\mathrm{pH}_{4}, 6$ and 9. The black dashed lines are the expected Debye lengths, the fitted Debye lengths and salt concentrations can be found in table 3.1.

\begin{tabular}{clllll} 
Expected conc. & 1 & 4 & 10 & 40 & 100 \\
\hline pH 4 & $0.9(10.1)$ & $3.9(4.9)$ & $10.3(3.0)$ & $42.0(1.5)$ & $103.5(0.9)$ \\
pH 6 & $1.3(8.4)$ & $4.5(4.5)$ & $09.0(3.2)$ & $39.6(1.5)$ & $125.3(0.8)$ \\
pH 9 & $1.4(8.1)$ & $4.6(4.5)$ & $11.0(2.9)$ & $40.2(1.5)$ & $096.5(1.0)$
\end{tabular}

Table 3.1: Ion concentration, in $m M$ (Debye length, in $\mathrm{nm}$ ), obtained from the curves in figure 3.4 


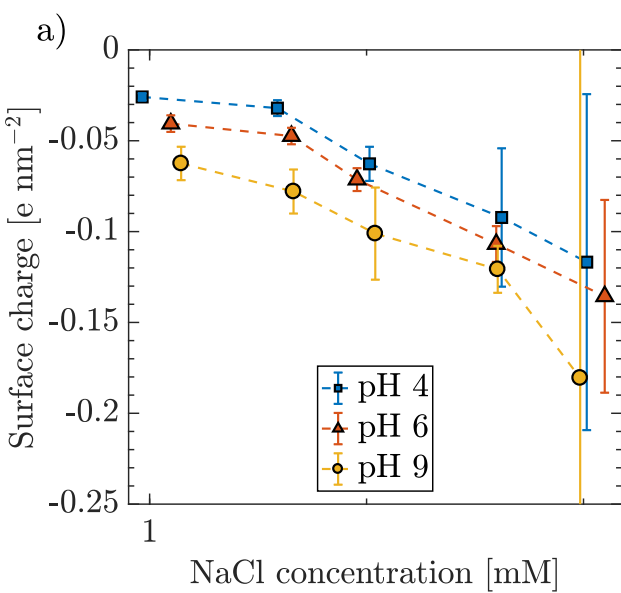

b)

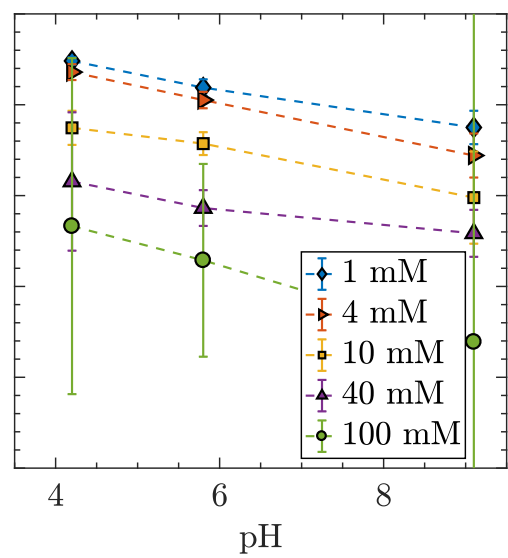

Figure 3.5: The net surface charge $\left(\sigma_{\mathrm{o}}+\sigma_{S}=-\sigma_{D}<0\right)$ of silica as function of a $) \mathrm{NaCl}$ concentration and b) $\mathrm{pH}$. For an increasing salt concentration and/or $\mathrm{pH}$ the absolute diffuse layer charge increases.

(the crossing with the $y$-axis) reveals a first estimate of the diffuse layer charge, which increases for increasing $\mathrm{pH}$. Eventually we extract the diffusive layer charge from the interaction stiffness curves shown in figure 3.4, using our exponential surface charge (ESC) model as described in chapter 2. The diffuse layer charge $\sigma_{D}$ neutralizes the intrinsic surface charge $\sigma_{\mathrm{o}}$ and the Stern layer charge $\sigma_{S}\left(\sigma_{\mathrm{o}}+\sigma_{S}=-\sigma_{D}<0\right)$. The results are shown as a function of concentration in figure $3.5 \mathrm{a}$ and as a function of $\mathrm{pH}$ in $3.5 \mathrm{~b}$. For increasing $\mathrm{pH}$, we observe an increasing diffuse layer charge. This is a consequence of charge regulation: when the concentration of the $\mathrm{H}^{+}$or $\mathrm{C}^{+}$ions increases, the equilibrium shifts towards a higher $\mathrm{H}^{+}$or $\mathrm{C}^{+}$adsorption, reducing the number of charged sites on the substrate. As expected, the diffuse layer charge clearly increases when the $\mathrm{pH}$ changes from 4 to 9 . A high $\mathrm{pH}\left(\right.$ i.e. low $\left[\mathrm{H}^{+}\right]$) favors the de-protonation of the silica substrate and the surface becomes more negatively charged. 


\begin{tabular}{cc}
\hline Reactions & Charge \\
\hline$S H \rightleftharpoons S^{-}+H^{+} ; K_{H}$ & $\sigma_{s}=\frac{-q_{e} \Gamma}{1+A \exp \left(-\frac{q_{e} \varphi_{s}}{k_{B} T}\right)}$ \\
$S C \rightleftharpoons S^{-}+C^{+} ; K_{C}$ & $A=10^{p K_{H}\left[H^{+}\right]_{\infty}+10^{p K_{C}}\left[C^{+}\right]_{\infty}}$ \\
$\Gamma=\left\{S^{-}\right\}+\{S H\}+\{S C\}$ &
\end{tabular}

Table 3.2: Surface chemistry equations and final charge regulation condition for the silica surface as used by a $2 p K$ surface complexation model $\left(p K_{X}=-\log _{10} K_{X}\right)$. The charge mentioned refers to the diffuse layer charge.

\section{Chemical interpretation}

With the use of the ESC model it is possible to determine the diffuse layer charge from measured data without any knowledge of the adsorption and desorption reactions. At this point we try to give a chemical interpretation of the measured data. As in previous work, a $2 \mathrm{pK}$ surface complexation model has been used to determine the chemical equilibrium constants $\mathrm{K}_{H}$ and $\mathrm{K}_{C}$ (table 3.2) of the surface speciation reactions of silica in contact with an electrolyte ${ }^{[42]}$. To do so, a merit function $Q$ has been defined to measure the agreement between experimental data and calculated model curves:

$$
Q_{j}=\frac{\sum_{i} N_{i j}}{\sum_{i} N_{i j} \chi_{i j}^{2}},
$$

where $N$ is the number of data points per averaged curved and $\chi^{2}=\frac{1}{N} \sum_{n=1}^{N}\left(P\left(z_{n}\right)-\right.$ $\left.P_{i}\right)^{2}$ the average of the squared differences between model curve and experimental value. Here $P_{i}\left(z_{n}\right)$ is the calculated (using the $2 \mathrm{pK}$ model) and $P_{j}$ the measured excess pressure at tip-substrate distance $z_{n}$. The indexes $i$ and $j$ represent the concentration and $\mathrm{pH}$ at which the merit function has been calculated. In figure 3.6 the merit functions for $\mathrm{pH}_{4}, 6$ and 9 are shown. These merit functions are acquired including all $\mathrm{NaCl}$ concentrations $(1,4,10,40,100 \mathrm{mM} \mathrm{NaCl})$. For each $\mathrm{pH}$, an optimum for $\mathrm{p} K_{H}$ can be found. For $\mathrm{pH} 6$ we obtain $\mathrm{p} K_{H}=6.4$, which is in line with the $\mathrm{pK}$ values found in literature. The $\mathrm{p} K_{C}$ values do not converge to a single value, but scatter over a broad range. This means that the modeling is not sensitive to the $\mathrm{pK}_{\mathrm{C}}$. 


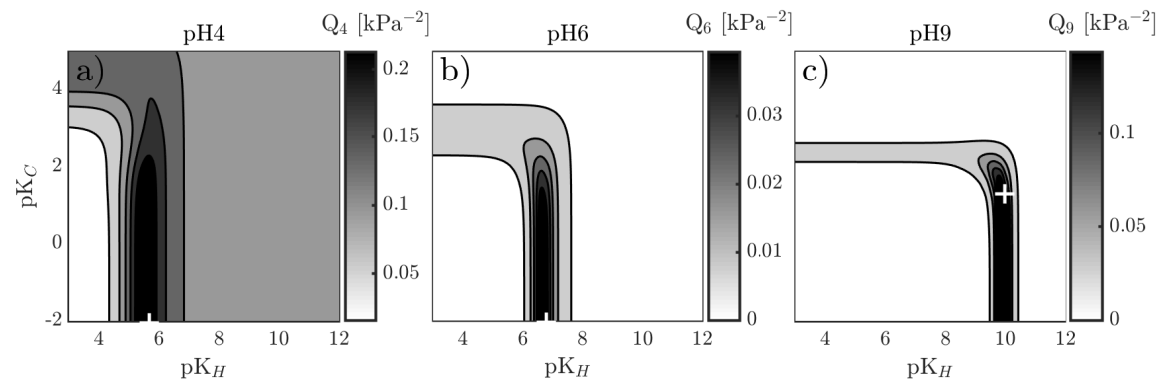

Figure 3.6: Contour plot of $Q_{j}($ for $j=4,6$ and 9$)$ in the parameter space $\left(p K_{H}, p K_{C}\right)$ for silica in contact with a $\mathrm{NaCl}$ solution at $\mathrm{pH} 4,6$ and 9. For the silica surface, local maxima, marked by the white cross, provide the best estimate of $p K_{H}=5 \cdot 3, p K_{C}=$ -2.0, $p K_{H}=6.4, p K_{C}=-2.0$ and $p K_{H}=9.6, p K_{C}=1.3$, respectively.

\subsection{Discussion}

\section{Diffuse layer charge density}

The above analysis shows that silica is negatively charged, typically from -0.02 to $-0.2 \mathrm{e} / \mathrm{nm}^{2}$. The diffuse layer charge increases with increasing $\mathrm{pH}$ and/or $\mathrm{NaCl}$ concentration. These results are similar to previous force spectroscopy results of our group $[42,40,43]$. The absolute diffuse layer charge values agree well, and also with the values obtained from electrokinetic measurements [9,26]. In our former investigations, relatively sharp tips $\left(R_{\text {tip }} \leq 50 \mathrm{~nm}\right)$ were used, whereas in this work we used colloidal probes with a radius of $750 \mathrm{~nm}$. A larger tip radius implies a larger interaction area, which increases the net force on the tip, thus the force sensitivity. Despite this increased sensitivity, the error in the diffuse layer charge at high concentrations remains relatively high. This is reflected in the error bars shown in figure 3.5. The Debye length is short at high salt concentrations and therefore only a small range is available for the charge fitting. Therefore, the uncertainty in the tip-sample distance 

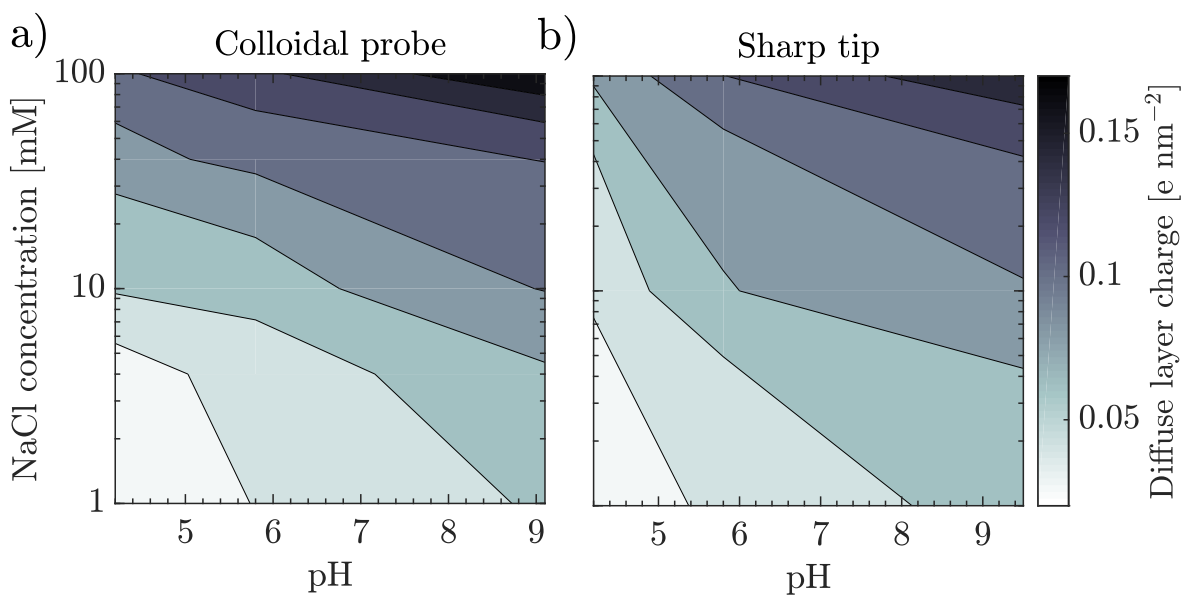

Figure 3.7: Contour plot of the diffuse layer charge as measured by a) colloidal probe and b) sharp tip consisting of 15 and 9 data points respectively.

causes a relatively high error in the diffuse layer charge values. When we compare the results from the colloidal probe with results obtained using sharp tips, see figure 3.7, we observe that the diffuse layer charge values are very similar to the colloidal probe results. Small differences can be caused by incomplete calibration of the sharp tip experiments, which did not undergo the extensive calibration procedure described here. The trends are also similar to the predictions made in the work of Zhao et al. [42]. So despite differences in the chemical modeling, the diffuse layer charge results for colloidal probe and sharp tip are consistent.

\section{Chemical interpretation}

From a broader perspective, the trends found here are similar to those found in titration measurements $[19,26,6,44]$; for increasing $\mathrm{pH}$ and constant ion concentrations the absolute intrinsic surface charge increases. However, for typical potentiometric titration measurements the absolute values are much higher, in the order of $1 \mathrm{e} / \mathrm{nm}^{2}$ (some exceptions: Kutzner et al. report values of $0.06 \mathrm{e} / \mathrm{nm}^{2}$ for this system [45] $)$. For electrokinetic or force measurements these values [9] lie around $0.1-0.2 \mathrm{e} / \mathrm{nm}^{2}$. Comparing numbers from both techniques, this suggests an adsorption of typically 
1 sodium ion per $\mathrm{nm}^{2}$.

To judge the validity of this suggestion for our study we reconsider the $2 \mathrm{pK}$ modeling. From the equilibrium constant estimation in figure 3.6, we find for each $\mathrm{pH}$ a clear optimum value for the $\mathrm{p} K_{H}$. Although the $\mathrm{pK}$ values should be independent of $\mathrm{pH}$ and ionic strength, we observe that this optimum does depend on $\mathrm{pH}$. Moreover, we do not find a specific optimum for $\mathrm{p} K_{C}$. Therefore, we had to conclude that this $2 \mathrm{pK}$ approach is insufficient to describe the full surface chemistry. This is in line with the observations of Zhao et al., where a distinct optimum was found for $\mathrm{p} K_{H}$ and $\mathrm{p} K_{\mathrm{C}}$ at $\mathrm{pH} 6^{\left[4^{42}\right]}$. Nevertheless, estimating $\mathrm{p} K_{H} \simeq 7$ and $\mathrm{p} K_{C} \simeq$ o, our limited model predicts for $10 \mathrm{mM}$ and $\mathrm{pH} 7$ an intrinsic surface charge $\sigma_{s}=-0.29 \mathrm{e} / \mathrm{nm}^{2}$, while the number of sodium ions in the Stern layer is approximately 0.08 ions $/ \mathrm{nm}^{2}$, which differs considerably from the above suggested 1 ion $/ \mathrm{nm}^{2}$. However, if we estimate $\mathrm{p} K_{C} \simeq 1$ instead of $\mathrm{o}$, we obtain $\sigma_{s}=-0.92 \mathrm{e} / \mathrm{nm}^{2}$, while the number of sodium ions in the Stern layer is approximately 0.71 ions $/ \mathrm{nm}^{2}$, which is quite close to the suggested 1 ion $/ \mathrm{nm}^{2}$. This shows once more the limitations of the $\mathrm{pK}$ modeling.

\subsection{Conclusion}

We used AFM colloidal probe force spectroscopy to probe the surface chemistry behavior of the silica surface in aqueous solutions of variable $\mathrm{pH}$ and $\mathrm{NaCl}$ concentration. Using an exponential surface charge (ESC) - potential relation we are able to extract diffuse layer charge values from the experimental force-distance curves. When using a $2 \mathrm{pK}$ model to determine the equilibrium constant for deprotonation of the silanol groups and that for adsorption of $\mathrm{Na}^{+}$cations, their values turn out to depend on the $\mathrm{pH}$ of the electrolyte solution. This indicates that a single $\mathrm{pK}$ pair cannot describe the surface chemistry of silica substrates adequately. However, the values for the diffuse layer charge, as obtained using the pK or ESC modeling and with sharp tips or colloidal probes, are all consistent.

Taking into account the considerations mentioned in the discussion section, we argue that the combination of Colloidal Probe Force Spectroscopy and diffuse layer charge fitting with the exponential surface charge model is a valuable method to determine the diffuse layer charge. However, linking these charge values to the relative 
occupation of surface sites is not reliable, because the chemical modeling of the substrate still fails. The obtained diffuse layer charge values can subsequently be used in further chemical modeling of the surface or for quantitative comparison with the results of other methods.

\section{Bibliography}

[1] C. A. J. Appelo and D. Postma, Geochemistry, groundwater and pollution. CRC press, 2004 .

[2] F. Mugele, I. Siretanu, N. Kumar, B. Bera, L. Wang, R. de Ruiter, A. Maestro, M. Duits, D. van den Ende, and I. Collins, "Insights from ion adsorption and contact-angle alteration at mineral surfaces for low-salinity waterflooding," SPE Journal, 2016.

[3] G. Sposito, The Environmental Chemistry of Aluminum, Second Edition. Taylor and Francis, 1995.

[4] B. Honig and A. Nicholls, "Classical electrostatics in biology and chemistry," Science, vol. 268, no. 5214, pp. 1144-1149, 1995.

[5] M.A. Brown, A. Goel, and Z. Abbas, "Effect of electrolyte concentration on the stern layer thickness at a charged interface," Angew Chem Int Ed Engl, vol. 55, no. 11 , pp. $3790-4,2016$.

[6] P. M. Dove and C. M. Craven, "Surface charge density on silica in alkali and alkaline earth chloride electrolyte solutions," Geochimica Et Cosmochimica Acta, vol. 69, no. 21 , pp. 4963-4970, 2005.

[7] S. Ben Jabrallah, F. Malloggi, L. Belloni, L. Girard, D. Novikov, C. Mocuta, D. Thiaudiere, and J. Daillant, "Electrolytes at interfaces: accessing the first nanometers using x-ray standing waves," Phys Chem Chem Phys, vol. 19, no. 1, pp. 167-174, 2016.

[8] K. Csoban and P. Joo, "Sorption of cr(iii) on silica and aluminium oxide: experiments and modelling," Colloids and Surfaces a-Physicochemical and Engineering Aspects, vol. 151, no. 1-2, pp. 97-112, 1999. 
[9] A. H. Jalil and U. Pyell, "Quantification of zeta-potential and electrokinetic surface charge density for colloidal silica nanoparticles dependent on type and concentration of the counterion: Probing the outer helmholtz plane," The Journal of Physical Chemistry C, vol. 122, no. 8, pp. 4437-4453, 2018.

[10] B. J. Kirby and E. F. Hasselbrink, "Zeta potential of microfluidic substrates: 1. theory, experimental techniques, and effects on separations," Electrophoresis, vol. 25, no. 2, pp. 187-202, 2004.

[11] M. Kosmulski, "The ph dependent surface charging and points of zero charge. vii. update," Adv Colloid Interface Sci, 2017.

[12] B. M. Lowe, C.-K. Skylaris, N. G. Green, Y. Shibuta, and T. Sakata, "Calculation of surface potentials at the silica-water interface using molecular dynamics: Challenges and opportunities," Japanese Journal of Applied Physics, vol. 57, no. 4 S, p. 04 FMo2, 2018.

[13] J. Lützenkirchen, T. Preočanin, D. Kovačević, V. Tomišić, L. Lövgren, and N. Kallay, "Potentiometric titrations as a tool for surface charge determination," Croatica Chemica Acta, vol. 85, no. 4, pp. 391-417, 2012.

[14] M. Schreier, T.E. Feltes, M. T. Schaal, and J. R. Regalbuto, "The determination of oxide surface charging parameters for a predictive metal adsorption model," J Colloid Interface Sci, vol. 348, no. 2, pp. 571-8, 2010.

[15] D. A. Sverjensky, "Prediction of surface charge on oxides in salt solutions: Revisions for 1:1 (m+l-) electrolytes," Geochimica et Cosmochimica Acta, vol. 69, no. 2, pp. 225-257, 2005.

[16] R. Vangara, D. C. R. Brown, F. van Swol, and D. N. Petsev, “Electrolyte solution structure and its effect on the properties of electric double layers with surface charge regulation," J Colloid Interface Sci, vol. 488, pp. 180-189, 2017.

[17] L. G. J. Fokkink, A. Dekeizer, and J. Lyklema, "Specific ion adsorption on oxides - surface-charge adjustment and proton stoichiometry," Journal of Colloid and Interface Science, vol. 118, no. 2, pp. 454-462, 1987. 
[18] J. Lyklema, "Quest for ion-ion correlations in electric double layers and overcharging phenomena," Advances in Colloid and Interface Science, vol. 147-48, pp. 205-213, 2009.

[19] T. Hiemstra, W. Van Riemsdijk, and G. Bolt, "Multisite proton adsorption modeling at the solid/solution interface of (hydr) oxides: A new approach: I. model description and evaluation of intrinsic reaction constants," Journal of colloid and interface science, vol. 133, no. 1, pp. 91-104, 1989.

[20] H.-J. Butt, K. Graf, and M. Kappl, Physics and chemistry of interfaces. Physics textbook, Weinheim: Wiley-VCH, 2003.

[21] J. A. Davis, R. O. James, and J. O. Leckie, "Surface ionization and complexation at the oxide/water interface: I. computation of electrical double layer properties in simple electrolytes," Journal of colloid and interface science, vol. 63, no. 3, pp. 480-499, 1978.

[22] S. J. T. Brugman, E. R. Townsend, M. M. H. Smets, P. Accordini, and E. Vlieg, "Concentration-dependent adsorption of csi at the muscovite-electrolyte interface," Langmuir, vol. 34, no. 13, pp. 3821-3826, 2018.

[23] A. A. Hassanali, H. Zhang, C. Knight, Y. K. Shin, and S. J. Singer, “The dissociated amorphous silica surface: Model development and evaluation," Journal of Chemical Theory and Computation, vol. 6, no. 11, pp. 3456-3471, 2010.

[24] J. Lutzenkirchen, A. Abdelmonem, R. Weerasooriya, F. Heberling, V. Metz, and R. Marsac, "Adsorption of dissolved aluminum on sapphire-c and kaolinite: implications for points of zero charge of clay minerals," Geochem Trans, vol. 15 , p. 9, 2014.

[25] M. Kobayashi, F. Juillerat, P. Galletto, P. Bowen, and M. Borkovec, "Aggregation and charging of colloidal silica particles: Effect of particle size," Langmuir, vol. 21 , no. 13 , pp. 5761-5769, 2005.

[26] M. Kobayashi, M. Skarba, P. Galletto, D. Cakara, and M. Borkovec, "Effects of heat treatment on the aggregation and charging of stober-type silica," J Colloid Interface Sci, vol. 292, no. 1, pp. 139-47, 2005. 
[27] F. J. Montes Ruiz-Cabello, G. Trefalt, P. Maroni, and M. Borkovec, "Electric double-layer potentials and surface regulation properties measured by colloidal-probe atomic force microscopy," Phys Rev E Stat Nonlin Soft Matter Phys, vol. 90, no. 1, p. $012301,2014$.

[28] A. V. Delgado, F. Gonzalez-Caballero, R. J. Hunter, L. K. Koopal, and J. Lyklema, "Measurement and interpretation of electrokinetic phenomena," Journal of Colloid and Interface Science, vol. 309, no. 2, pp. 194-224, 2007.

[29] R.J. Hill, D. A. Saville, and W. B. Russel, "Electrophoresis of spherical polymercoated colloidal particles," Journal of Colloid and Interface Science, vol. 258, no. 1, pp. 56-74, 2003.

[30] R. W. Obrien and L. R. White, "Electrophoretic mobility of a spherical colloidal particle," Journal of the Chemical Society-Faraday Transactions Ii, vol. 74, pp. $1607-1626,1978$.

[31] V. Lobaskin, B. Dunweg, M. Medebach, T. Palberg, and C. Holm, "Electrophoresis of colloidal dispersions in the low-salt regime," Physical Review Letters, vol. 98, no. 17, 2007.

[32] J. N. Israelachvili, "Measurement of forces between surfaces immersed in electrolyte-solutions," Faraday Discussions, vol. 65, pp. 20-24, 1978.

[33] R. M.Espinosa-Marzal, T. Drobek, T. Balmer, and M. P. Heuberger, "Hydratedion ordering in electrical double layers," Physical Chemistry Chemical Physics, vol. 14, no. 17, pp. 6085-6093, 2012.

[34] S. H. Behrens and M. Borkovec, "Electric double layer interaction of ionizable surfaces: Charge regulation for arbitrary potentials," Journal of Chemical Physics, vol. 111, no. 1, pp. 382-385, 1999.

[35] I. Popa, P. Sinha, M. Finessi, P. Maroni, G. Papastavrou, and M. Borkovec, "Importance of charge regulation in attractive double-layer forces between dissimilar surfaces," Physical Review Letters, vol. 104, no. 22, 2010.

[36] G. Trefalt, S. H. Behrens, and M. Borkovec, "Charge regulation in the electrical double layer: Ion adsorption and surface interactions," Langmuir, 2015. 
[37] F. Adekola, M. Fedoroff, H. Geckeis, T. Kupcik, G. Lefevre, J. Lutzenkirchen, M. Plaschke, T. Preocanin, T. Rabung, and D. Schild, "Characterization of acid-base properties of two gibbsite samples in the context of literature results," Journal of Colloid and Interface Science, vol. 354, no. 1, pp. 306-317, 2011.

[38] M. Szekeres and E. Tombácz, "Surface charge characterization of metal oxides by potentiometric acid-base titration, revisited theory and experiment," Colloids and Surfaces A: Physicochemical and Engineering Aspects, vol. 414, pp. 302313, 2012.

[39] S. H. Behrens and D. G. Grier, “The charge of glass and silica surfaces,” Journal of Chemical Physics, vol. 115, no. 14, pp. 6716-6721, 2001.

[40] F. Liu, S. de Beer, D. van den Ende, and F. Mugele, "Atomic force microscopy of confined liquids using the thermal bending fluctuations of the cantilever," Physical Review E, vol. 87, no. 6, p. 062406, 2013.

[41] F. Liu, C. L. Zhao, F. Mugele, and D. van den Ende, "Amplitude modulation atomic force microscopy, is acoustic driving in liquid quantitatively reliable?," Nanotechnology, vol. 26, no. 38, 2015.

[42] C. L. Zhao, D. Ebeling, I. Siretanu, D. van den Ende, and F. Mugele, "Extracting local surface charges and charge regulation behavior from atomic force microscopy measurements at heterogeneous solid-electrolyte interfaces," Nanoscale, vol. 7, no. 39, pp. 16298-16311, 2015.

[43] A. Klaassen, F. Liu, D. van den Ende, F. Mugele, and I. Siretanu, "Impact of surface defects on the surface charge of gibbsite nanoparticles," Nanoscale, 2017.

[44] T. A. Gmür, A. Goel, and M. A. Brown, "Quantifying specific ion effects on the surface potential and charge density at silica nanoparticle-aqueous electrolyte interfaces," The Journal of Physical Chemistry C, vol. 120, no. 30, pp. 16617$16625,2016$.

[45] S. Kutzner, M. Schaffer, T. Licha, E. Worch, and H. Bornick, "Sorption of organic cations onto silica surfaces over a wide concentration range of competing electrolytes," Journal of Colloid and Interface Science, vol. 484, pp. 229-236, 2016. 


\section{4}

\section{Impact of surface defects on the surface charge of gibbsite nanoparticles ${ }^{1}$}

We USe high Resolution Atomic Force Microscopy to study the effective surface charge of the basal plane of gibbsite nanoparticles, with a lateral resolution of approximately $5 \mathrm{~nm}$, in ambient electrolyte of variable $\mathrm{pH}$ and salt content. Our measurements reveal surface charge variations on the basal planes that correlate with the presence of topographic defects such as atomic steps. This surface charge heterogeneity, which increases with increasing $\mathrm{pH}$, suggests that for a $\mathrm{pH}$ between 6 and 9 the defect sites display a stronger chemical activity than adjacent, apparently atomically smooth regions of the basal plane. Smooth regions display a slight positive net surface charge of $\approx 0.05 \mathrm{e} / \mathrm{nm}^{2}$ that hardly varies within this $\mathrm{pH}$ range. In contrast, near the topographic defects we observe a much lower charge. Considering the size of the interaction area under the probing tip, this implies that at the defect sites the

\footnotetext{
${ }^{1}$ The results described in this chapter have been published as: Klaassen, A, Nanoscale 2017, 9, 4721-4729
} 
charge density must be negative, $\approx-0.1 \mathrm{e} / \mathrm{nm}^{2}$. These measurements demonstrate that surface defects have a large influence on the average surface charge of the gibbsite basal plane. These findings will contribute to understanding why surface defects play an important role in various applications, such as fuel cells, chemical synthesis, self-assembly, catalysis and surface treatments.

\subsection{Introduction}

Clays and mineral nanoparticles in general are important for many natural phenomena and technological processes. They play a crucial role in the development of building materials, the transport of pollutants through groundwater and soil, the retention of nuclear waste, and the recovery of crude oil from geological reservoirs [1]-[5]. Essential for their crucial role in these applications is their ability to bind organic and inorganic solutes from their surroundings, e.g. from ambient water. This ability, in turn, is governed - at least to a certain extent - by the charge on the surface of the particles. Extensive efforts have therefore been devoted to the characterization and microscopic understanding of the charging behavior of these clays and mineral nanoparticles in aqueous solution ${ }^{[6-10]}$. Classically, such experiments involve titration experiments, e.g. potentiometry and electrokinetic measurements, involving macroscopic amounts of particle suspensions $[11-19]$. The macroscopic character of such measurements has the advantage of providing a good statistical average over many particles. At the same time, averaging implicates a disadvantage when trying to understand the microscopic aspects of the charging mechanisms involved. Slow equilibration, different response of different crystallographic facets with poorly known relative surface areas, and poor knowledge of the microscopic properties of each facet - due to differences in mineral sources and preparation protocols - all contribute to a substantial uncertainty and variation in the results obtained by different laboratories on nominally identical materials $[7,[15,16,18-21]$. A striking example is the reported values of the point of zero charge (PZC) that ranges from 6 to 11 [7, 22-24]

From this perspective, it is not surprising that a consistent interpretation of the charging behavior in terms of microscopic surface speciation models, molecular dynamics (MD) [25] simulations or density functional theory (DFT) is not possible.

In recent years, the advent of high resolution atomic force microscopy (AFM) has provided new opportunities to study mineral-electrolyte interfaces with atomic reso- 
lution $[6,8,26,27]$. These measurements revealed details of the surface structure, the solvation water molecules, adsorbing ions, and the adjacent electric double layer. Thanks to the small scales involved and the focus on perfectly defined model systems, high-resolution measurements enable a direct comparison with atomistic simulations and thus contribute to a truly microscopic understanding of the complex structure of such interfaces. Recently, we reported surface charge measurements for (natural) kaolinite particles deposited on mica and sapphire substrates, where on mica the silica-like, and on sapphire the gibbsite-like facet is exposed to the ambient electrolyte ${ }^{[26]}$. At $\mathrm{pH} \approx 5.8$, the silica-like basal plane displayed an average negative surface charge density $\left(\approx-0.04 \mathrm{e} / \mathrm{nm}^{2}\right)$ and the gibbsite facet a weak positive charge density of approximately $0.005 \mathrm{e} / \mathrm{nm}^{2}$, corresponding roughly to one positive unit charge per 40 surface unit cells. Our measurements also displayed substantial lateral variations in this charge density on topographically rather smooth facets. Considering theoretical studies, there is a general consensus that the charge of gibbsite particle arises primarily from singly coordinated aluminol ( $\equiv \mathrm{AlOH})$ sites on the edge of the particle $[13,25,28]$. Whether the gibbsite basal plane (001) is charged or not, is not clear. According to the classical multisite surface complexation (MUSIC) model of Hiemstra et al., the doubly coordinated aluminol groups $\left(\equiv \mathrm{Al}_{2} \mathrm{OH}\right)$ on the basal plane (that is supposed to be atomically flat) are chemically inactive for $\mathrm{pH}<$ ${ }_{10}[28-31]$. Therefore the gibbsite surface charge was interpreted as being caused by "defects" on the basal plane (oo1), that can introduce active sites on the basal plane. On the other hand, more sophisticated models that take into account the existence of six crystallographically different $\mathrm{OH}$ - groups in each unit cell of the basal surface, predict that one out of these six should have a $\mathrm{pK}$ value between 5 and 6 . This $\mathrm{OH}^{-}$ group will be active within the $\mathrm{pH}$ range of typical experiments [9, 32, 33]. These calculations are supported by recent experiments of Rosenqvist et al. [12] and Gan and Franks [6], who observed a positive charge density on the basal plane of the gibbsite particles. Based on these experiments, and in contradiction with Hiemstra et al., they suggested that the doubly coordinated aluminol groups on the basal surface can (de)protonate and form uncharged complexes with ions from the electrolyte. Yet, current experimental evidence is also inconclusive for several reasons. Firstly, the experiments by Gan and Franks were carried out using the colloidal probe AFM technique ${ }^{[6]}$. This technique averages laterally over an area of several micrometer squared. Even freshly cleaved gibbsite crystals of good quality can contain many surface defects with lateral dimensions of a few nanometers [27]. Such defects are very 
common on clay and mineral surfaces of variable provenance [27]. Therefore, (as suggested by Hiemstra et al.) depending on their surface chemistry and density, defects may dominate the apparent surface charge measured in a colloidal probe AFM measurement, and thereby suggest a different surface chemistry of the basal plane than the true intrinsic one (be responsible for the experimentally observed charge density). Secondly, Zhao et al. [34] recently demonstrated that AFM spectroscopy measurements, usually do not contain sufficient information to discriminate between competing surface complexation models for the gibbsite basal plane, even if the regulation of surface charges upon approaching the AFM towards the surface is properly taken into account [34]. AFM spectroscopy, like electrokinetic techniques, is only sensitive to the charge in the diffuse layer. Any surface complexation model that leads to the same charge density in the diffuse layer results in identical force-distance curves. Reliable discrimination between competing models is therefore not possible. For this reason, we will restrain our analysis in the present work to the determination of local surface charge values, rather than attempting to identify specific surface complexation reactions [34,35]. Summarizing, the origin and the amount of surface charge on gibbsite, like for many other minerals and clays, is not well established $[30,28,36-38]$. Moreover, understanding of the impact of defects/surface singularities on surface properties of clay particles in general is an important issue in material science. Defects, like dislocations, vacancies and steps are omnipresent at material surfaces and play an important role in the chemical reactivity and properties of these surfaces. A thorough characterization of complex surface-imperfections is already challenging for surfaces in vacuum, in particular if large-scale defects or combinations of different defects are involved. In case of solid-liquid interfaces, the complexity is further increased by the mutual interplay between surface features and the solvent. Investigation of surface defects in the presence of water/electrolytes will be beneficial for a wide range of interfacial processes, as occur in electrochemical processes, catalysis, food processing, health science, chemical synthesis, and surface treatment. The goal of the present work is to identify whether the basal plane of gibbsite does carry charge and if so, to determine whether this charge density is $\mathrm{pH}$-responsive and to what extent it can be related to the presence of defects on the surface. To this end, we performed dynamic AFM spectroscopy measurements in ambient aqueous electrolytes on individual nanoparticles of gibbsite adsorbed onto an oxidized Si wafer, while also imaging the height profiles of these particles. To minimize the possibility of intrinsic structural surface charges due to isomorphous substitution, we use synthetic gibbsite 
particles for our measurements [17]. Individually selected particles are monitored for several hours while the $\mathrm{pH}$ and salt content of the solution are varied. We determine tip-sample interaction forces as a function of approach distance and extract the surface charges with a lateral resolution of about ten nanometers. This resolution is determined by the contact area between the tip and the probed surface (of the order of $100 \mathrm{~nm}^{2}$ ). At this length scale, the surface can still be described by conventional continuum physics, including in particular a continuum surface charge density rather than a fully discrete distribution of ions. While larger than the surface unit cell, this scale is still much smaller than the typical lateral dimensions $(\mathrm{O}(200 \mathrm{~nm}))$ of our nanoparticles which enables an investigation of the lateral variation of the charge density along their surface. The local surface charge is extracted from the measured force-distance curves using the DLVO (Derjaguin, Landau, Verwey, Overbeek) theory including charge regulation.

\subsection{Methods}

\section{Sample and probe preparation}

The sample and cantilever is cleaned following the procedure described in chapter 3.2.1. In addition, gibbsite from a gibbsite stock suspension (in $20 \mathrm{mM} \mathrm{NaCl}$ ) provided by the research group of A. Philipse ${ }^{[17]}$ (University of Utrecht, The Netherlands) was used. A $10 \mu \mathrm{L}$ drop of a 100 times diluted gibbsite suspension is placed on the cleaned silicon wafer. After a residence time of $60 \mathrm{~s}$, in which the particles can settle on the substrate, the excess suspension is removed and the substrate is dried with a flow of air. Then, the sample is rinsed with copious amounts of deionized water and dried with a flow of nitrogen.

For the measurements, rectangular cantilevers (MikroMash $\mathrm{NSC}_{3} 6 / \mathrm{Cr}-\mathrm{Au} \mathrm{BS}$ ) are used and cleaned following the procedure in chapter 3.2.1.

\section{Amplitude modulation force spectroscopy}

Dynamic force spectroscopy measurements were performed with a commercial Asylum Research Cypher ES equipped with photo thermal excitation [40,41]. Photother- 
mal excitation does not suffer from the 'forest of peaks' in the transfer function [42] and liquid born excitation [43,44] as known for (piezo driven) acoustic excitation. This simplifies the force extraction considerably. Another advantage concerns the cantilever amplitude, which is stable over several hours. All experiments are performed under fluid, in a closed fluid cell that allows solvent exchange and is kept at a constant temperature of $29.7 \pm 0.5^{\circ} \mathrm{C}$. Fluid exchange is accomplished by simultaneously injecting a new solution and removing the old solution. For every exchange, about 25 times of the total drop volume present in the fluid cell is replaced. The fresh solution is allowed to equilibrate for $10-15 \mathrm{~min}$ before starting data acquisition. The AFM is used in two operational modes, amplitude modulation (AM) imaging mode and force spectroscopy. In AM mode, topography images of the sample are taken, which provide information regarding the size and topography of the particles on the substrate. Force spectroscopy is performed using the force volume map functionality provided by the Cypher MFP-3D software. In this mode, the mean deflection $(u)$, amplitude $(A)$, phase $(\varphi)$ and drive frequency $\left(\omega_{d}\right)$ versus the measured piezo position $\left(z_{p}\right)$ are recorded in a $2 \mathrm{D}$ grid over the area of interest. This results in a $3 \mathrm{D}$ volume of data, where each position on the $2 \mathrm{D}$ grid is an approach curve. Figure 4.1 a shows an example of the amplitude and phase shift curves on a silica surface. To minimize changes in the shape of the tip apex during the spectroscopy measurements, we did not allow the amplitude signal to drop below a chosen threshold ( $80 \%$ of amplitude) of its free amplitude value $(\approx 2 \mathrm{~nm})$.

\section{Force inversion}

The conversion of amplitude and phase into the tip-sample interaction force is described in detail by de Beer et al. [45] and Liu et al. [44,46] for acoustic driven cantilevers. We use photothermal excitation; therefore, we must slightly modify the force inversion procedure. The laser beam, which is used to drive the cantilever, has a constant intensity with a sinusoidal modulation on top. The laser intensity I induces an added deflection of the cantilever, therefore the relation between the force $\mathrm{F}$ and tip displacement z given by $F=k_{c}\left(z-z_{T}\right)$. Here, $z_{T}$ is the zero-load deflection due to the temperature increase caused by the laser and $k_{c}$ is the effective stiffness of the cantilever. According to linear response theory $z_{T}$ can be written for small thermal variations as $z_{T}(t)=\int_{0}^{\infty} A\left(t^{\prime}\right) I\left(t-t^{\prime}\right) d t$ or in the frequency domain as 

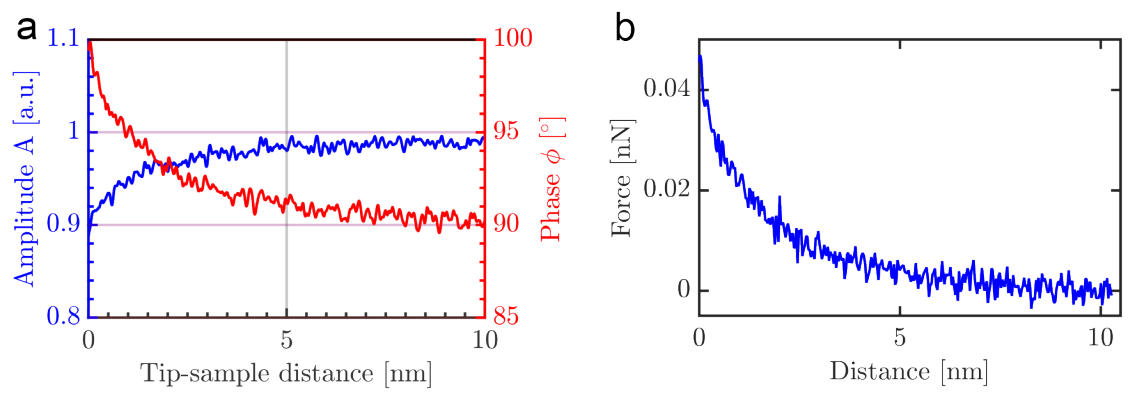

Figure 4.1: (a) Amplitude and phase data recorded on silica in a $10 \mathrm{mM} \mathrm{NaCl} \mathrm{pH} 6$ solution during the force volume method. The amplitude is normalized to the amplitude at $20 \mathrm{~nm}$ from the surface $A_{\infty}$. (b) Amplitude and phase are converted to the interaction force using equation 4.3. Experimental parameters in liquid: Mikromash $\mathrm{NSC}_{3} 6 / \mathrm{Au}-\mathrm{BS}$, $k_{c}=0.64 \mathrm{~N} \mathrm{~m}^{-1}, \omega_{\infty}=21.6 \mathrm{kHz}, Q=2.8$.

$z(\omega)=A_{T}^{*}(\omega) I_{\circ}$. For small amplitudes, the displacement of the cantilever tip can be described and modeled as a damped simple harmonic oscillator:

$$
m^{*} z+\gamma_{c} z+k_{c} z=k_{c} z_{T}(t)+F_{t s}(h+z, z)
$$

where $m^{*}=k_{c} / \omega_{\mathrm{o}}^{2}$ is the effective mass of the cantilever including the added mass due to the surrounding liquid, $\omega_{\mathrm{o}}$ the resonance frequency, $\gamma_{c}=k_{c} /\left(\omega_{\mathrm{o}} Q\right)$ the damping coefficient, $Q$ the quality factor and $F_{t s}$ the tip-sample interaction force. When the amplitude of the tip oscillation is small compared to the characteristic interaction length, the tip-sample interaction force can be linearized and rewritten as $F_{t s}(h+z, z)=F_{t s}(h, o)-k_{i n t} z-\gamma_{\text {int }} z$ Here $k_{\text {int }}=\partial F_{t s} / \partial h$ is the interaction stiffness, $\gamma_{\text {int }}$ the interaction damping coefficient and $F_{t s}(h, o)$ the equilibrium force at dis-

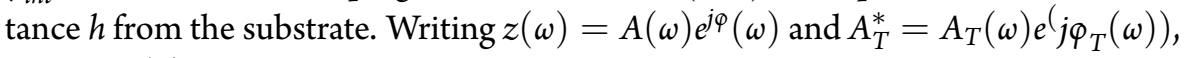
equation (1) is in the frequency domain equivalent to:

$$
\frac{A e^{j \varphi}}{I_{\mathrm{o}}}=\frac{k_{c} A_{T} e^{j \varphi_{T}}}{\left(k_{c}-m^{*} \omega^{2}+j \gamma_{c} \omega\right)+k_{i n t}+j \gamma_{i n t} \omega},
$$

From this equation, the force inversion equation can be derived. To eliminate the unknown factor $A_{T} e^{j \varphi_{T}}$ we use the ratio $A / A_{\infty} e^{j}\left(\varphi-\varphi_{\infty}\right)$, where $\left.A_{\infty} e^{\left(j \varphi_{\infty}\right.}\right)$ is 

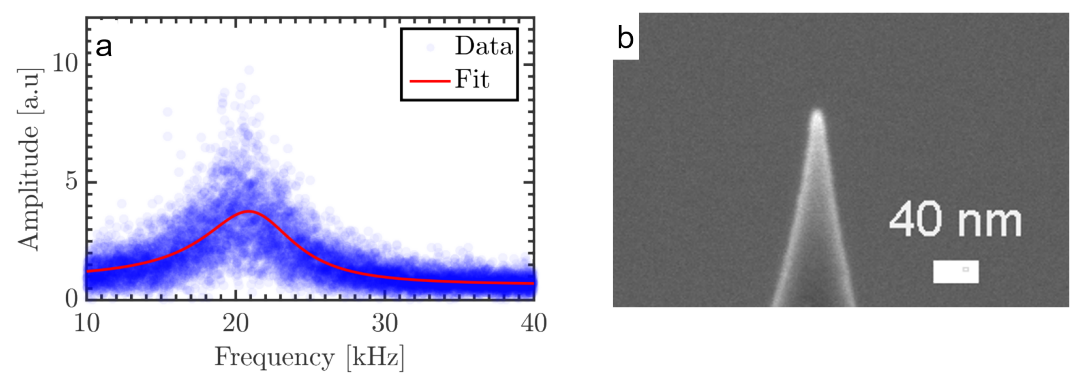

Figure 4.2: (a) The power density spectrum of the cantilever as result of thermal fluctuations recorded in liquid at a distance where the tip-sample interaction is considered zero. In red the fit curve, from which the quality factor and resonant frequency are extracted. In this experiment $Q=2.8$ and $\omega_{\infty}=21.0 \mathrm{kHz}$. (b) Scanning electron microscope image of the cantilever after being used in the experiment. The tip radius is estimated to be around $5 \mathrm{~nm}$.

measured at $h=20 \mathrm{~nm}$ at which distance both $k_{\text {int }}$ and $\gamma_{\text {int }}$ are negligibly small. Extracting $k_{\text {int }}$, equation 4.2 leads to:

$$
k_{\text {int }}=k_{\mathcal{c}}\left[1-\left(\frac{\omega}{\omega_{\mathrm{o}}}\right)^{2}\right] \frac{\left.A_{\infty} \cos \left(\varphi-\varphi_{\infty}\right)-A\right)}{A}+\gamma_{c} \omega \frac{A_{\infty} \sin \left(\varphi-\varphi_{\infty}\right)}{A} . \quad \text { (4.3) }
$$

Eventually, integrating equation 4.3 leads to the desired tip-sample interaction force. An example of the conversion of amplitude and phase into force can be found in figure $4.1 \mathrm{~b}$ and 4.3 . In equation 4.3 the cantilever parameters $k_{c}, \gamma_{c}$ and $m^{*}$ are unknown and have to be determined during the experiment. The stiffness $k_{c}$, quality factor $Q$ and eigenfrequency $\omega_{0}$, are extracted from the thermal noise spectrum of the cantilever in liquid at $h=20 \mathrm{~nm}$, where the tip-sample interaction is negligible (Figure 4.2 a). Using these properties, $m^{*}$ and $y_{c}$ can be determined (see text near equation 4.2. ). The size of the cantilever tip is determined using Scanning Electron Microscopy (Figure 4.2a). Typical experimental values are $k_{c} \approx{ }_{3} \mathrm{~N} \mathrm{~m}^{-1}, \omega_{0} \approx 28 \mathrm{kHz}, \mathrm{R} \approx$ $10 \mathrm{~nm}, \mathrm{Q} \approx 3$. All cantilever properties mentioned in this work are determined in liquid, except the tip radius, which is imaged after the experiment. 


\section{Surface charge calculation}

We use DLVO-theory, including van der Waals and electric double layer forces and a charge regulation model for tip and substrate surface chemistry, to extract the diffuse layer charge $\left(-\sigma_{D}=\sigma_{\mathrm{o}}+\sigma_{S}\right)$ from the force-distance curves $[26,34,47,35,48]$. This procedure has been explained in detail by Zhao et al. [34]. Here we will only give the final expression for the effective charge density $\sigma_{\text {eff }}$ as a function of the surface potential $\varphi_{s}$ for silica and gibbsite as obtained from the charge regulation model (table 4.1). In the evaluation of $\sigma_{s}\left(\varphi_{s}\right)$ and the interaction force, we need values for the parameters $A_{H}, \gamma, \mathrm{K}_{\mathrm{H}_{1}}, \mathrm{~K}_{\mathrm{H}_{2}}, \mathrm{~K}_{\mathrm{A}}$ and $\mathrm{K}_{\mathrm{C}}$. The Hamaker constant $A_{H}$ and the site density $\gamma$ are taken from literature $[6,49,50]$. The Hamaker constants are fixed to $0.65 \times 10^{-20} \mathrm{~J}$ for the (silica-water-silica) system and to $1.2 \times 10^{-20} \mathrm{~J}$ for the (silica-water-gibbsite) system ${ }^{[8]}$. The site densities of silica and gibbsite are set to 8 and 13.8 sites $/ \mathrm{nm}^{2}$, respectively $[30]$. The values for the equilibrium constant $\mathrm{K}_{i}$ are determined by fitting the calculated force curves to the experimental data. In order to extract the diffuse charge from an asymmetric system (silica-water-gibbsite) we first need to characterize the charge on the tip. This is done by measuring the tip-sample interaction force for a symmetric system (silica-water-silica) for each electrolyte condition. Once the charge of the tip is known, the charge on gibbsite can be determined. This procedure results in accurate values for the surface charge densities on both surfaces, but the $\mathrm{K}_{i}$ values of the surface reactions are less accurate. As explained in Zhao et al. [34], any combination of surface reactions that generates the same surface charge gives rise to the same interaction force in the AFM measurement. A reliable measurement of the $\mathrm{K}_{i}$ values (or $\mathrm{pK}_{i}$ values, $\mathrm{pK}_{i}=-\log \mathrm{K}_{i}$ ) based on force-distance curves for a limited set of fluid compositions is impossible when several surface reactions occur simultaneously. Therefore, we will only discuss the effective surface charge density $\left(\sigma_{\text {eff }}=\sigma_{\mathrm{o}}+\sigma_{S}\right)$ on the surfaces and not $\mathrm{pK} \mathrm{K}_{i}$ values. An example of the fitting routine can be found in figure $4.3 \mathrm{a}$.

\section{Error analysis}

In this section, we discuss the influence of several parameters on the accuracy of the surface charge determination. First, we consider the distance range in which the forces are analyzed. In figure 4.3 we show interaction force-distance curves calcu- 


\begin{tabular}{cc}
\hline Silica & Gibbsite \\
\hline$S H \rightleftharpoons S^{-}+H^{+} ; K_{H_{1}}$ & $S H_{2}^{+} \rightleftharpoons S H+H^{+} ; K_{H_{2}}$ \\
$S C \rightleftharpoons S^{-}+C^{+} ; K_{C}$ & $S H_{2} A \rightleftharpoons S H_{2}^{+}+A^{-} ; K_{A}$ \\
$\Gamma=\left\{S^{-}\right\}+\{S H\}+\{S C\}$ & $\Gamma=\left\{S^{-} S H_{2}^{+}\right\}+\{S H\}+\left\{S H_{2} A\right\}$ \\
$-e \Gamma$ & $-e \Gamma$ \\
$\left.\sigma_{s}=-\frac{\left[H^{+}\right]_{\infty}}{K_{H_{1}}}+\frac{\left[C^{+}\right]_{\infty}}{K_{C}}\right) e^{-\frac{e \varphi_{s}}{k_{B} T}}$ & $\sigma_{s}=-\frac{e-\left(\frac{K_{H_{2} \infty}}{\left[H^{+}\right]_{\infty}}+\frac{\left[A^{-}\right]_{\infty}}{K_{A}}\right) e^{-\frac{e \varphi_{s}}{k_{B} T}}}{1+\left(\frac{\left[H^{2}\right.}{}\right.}$ \\
\hline
\end{tabular}

Table 4.1: Surface speciation reactions, site densities and charge for silica and gibbsite.
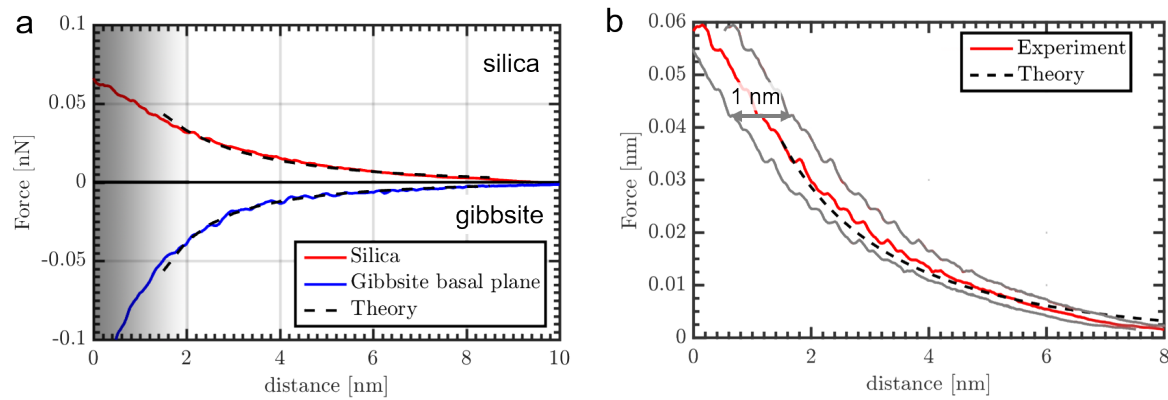

Figure 4.3: (a) Plot of the electrostatic part of the tip sample interaction force on silica and gibbsite in a $10 \mathrm{mM} \mathrm{NaCl} \mathrm{pH} 9$ solution. The charge is calculated using DLVO theory with charge regulation boundary conditions. The charge and $p K$ values resulting from this fitting for silica are $p K_{H}=1.4, p K_{C}=2.3, \sigma=-0.173 \mathrm{e} / \mathrm{nm}^{2}$ and gibbsite $p K_{H_{2}}=5.0, p K_{A}=2.1$, $\sigma=0.056 \mathrm{e} / \mathrm{nm}^{2}$ (b) Calculation of the effect of the uncertainty in zero on the surface charge. We fit the surface charge for 32 approaches while we shift each tip-sample distance by -0.5 and $0.5 \mathrm{~nm}$ (Figure $4.1 \mathrm{~b}$ ). We calculate the mean error for both shifts, which results in a $20 \%$ error in the average surface charge. (Tip parameters in liquid: $Q=2.8, \omega_{\infty}=$ $\left.21.6 \mathrm{kHz}, \omega_{d}=20.2 \mathrm{kHz}, A_{\mathrm{o}}=1.7 \mathrm{~nm}, k=0.64 \mathrm{~N} \mathrm{~m}^{-1}, R \approx 5 \mathrm{~nm}\right)$. 
lated with the DLVO theory and the charge regulation model (black lines) and from which the surface charge and the surface potential of the silicon oxide and gibbsite surfaces are determined. The lower limit for the fitting range is set to $1.5 \mathrm{~nm}$, in order to minimize the influence of short range forces, since they are not included in the model. The upper boundary was set to $9 \mathrm{~nm}$, above which the tip-sample interaction force is negligible. Changing the upper limit of the fitting boundary to $10 \mathrm{~nm}$ leads to a $1 \%$ change in the resulting charge. Variation of the lower limit from 1 to $3.5 \mathrm{~nm}$ results in a maximum of $15 \%$ change in the surface charge. Other parameters that can introduce inaccuracies in the surface charge calculation are: the instrument sensitivity and the choice of the absolute zero on the distance scale. Practically, these two parameters can be extracted from a force curve recorded in contact mode. The linear part of the force curve in the 'contact regime' is assumed to be the zero distance (separation) and its slope determines the sensitivity expressed in $\mathrm{Vnm}^{-1}$. This calibration needs to be done for each type of sample surface and electrolyte condition. Since we make use of heterogeneous samples, this procedure needs to be done for each approach, which is not possible. Furthermore, it is undesirable to bring the cantilever tip into hard contact with the sample, since we want to minimize changes of the tip apex during the spectroscopy measurements. Also, hard contact can lead to the contamination of the tip. Therefore, in our experiments, the 'o' on the distance axis was taken at the point where the amplitude is $80 \%$ of its free amplitude. The uncertainty in zero leads to an error in the absolute values obtained from the surface charge fitting. To estimate how large this error is, we fit the surface charge for 32 approaches while we shift each tip-sample distance by -0.5 and $0.5 \mathrm{~nm}$ (Figure 4.3 b). We calculate the mean error for both shifts, which results in a $20 \%$ error in the average surface charge. Additionally, imperfections in the geometry of the tip, surface roughness, size of the tip comparing to Debye length, etc., can cause small deviations from the force curves in theoretical models [50,51], which are more hard to quantify. 


\subsection{Results}

\subsubsection{AM-AFM imaging of gibbsite nanoparticles}

Figure 4.4 a shows an AFM height image of well-formed gibbsite crystals deposited on a silica substrate. The nanoparticles display the characteristic hexagonal plateletlike morphology and expose their (oo1) basal plane to the solution. Some of the gibbsite basal planes look skewed, which is a result of the crystal growth occurring during the synthesis of the particles [52]. The particle height varies from 5 to $20 \mathrm{~nm}$, and the lateral dimensions range from 100 to about $500 \mathrm{~nm}$, as shown in the line profile of figure $4.4 \mathrm{c}$. Figure $4.4 \mathrm{~b}$ shows a zoomed view of a single particle. Due to the higher resolution, the image reveals more topographical features, such as regions with a width of a few tens of nanometers with a smooth topography (terraces), separated by areas which are more irregular, containing steps and various other defects. Furthermore, mono- to multi-atomic steps are found on the basal plane (Figure $4.4 \mathrm{~d}$ ). Overall, the number of surface imperfections is enhanced near the rim of the particle. The surface imperfections near the center of the basal plane and those close to the edge of the particle are very characteristic for gibbsite particles, and are observed on many other particles in other independent experiments (Figure 4.5). In principle, one can obtain more detailed information about the surface structure by imaging these particles with atomic resolution, as shown in our previous work [27]. In those images we observe, next to regions of perfect crystallinity, extended regions of various types of topographic defects on the surfaces. These topographic defects include vacancies of one or a few atoms, vacancy islands, atomic steps, regions that show an imperfect lattice, etc. However, presently we cannot combine atomic resolution imaging with force spectroscopy. In this study, we will use the term defect for any topological deviation from the regular geometrical arrangement of the atoms in their crystalline lattice.

\subsection{2 $3 D$ force field measurements}

Figure 4.6a shows a 3 dimensional (3D) map of the force-distance curves of a silicagibbsite system in a $10 \mathrm{mM} \mathrm{NaCl}$ solution at $\mathrm{pH}$ 9. The lateral distance between each force-distance curve is $2.3 \mathrm{~nm}$. The red and blue colors represent repulsive and attrac- 

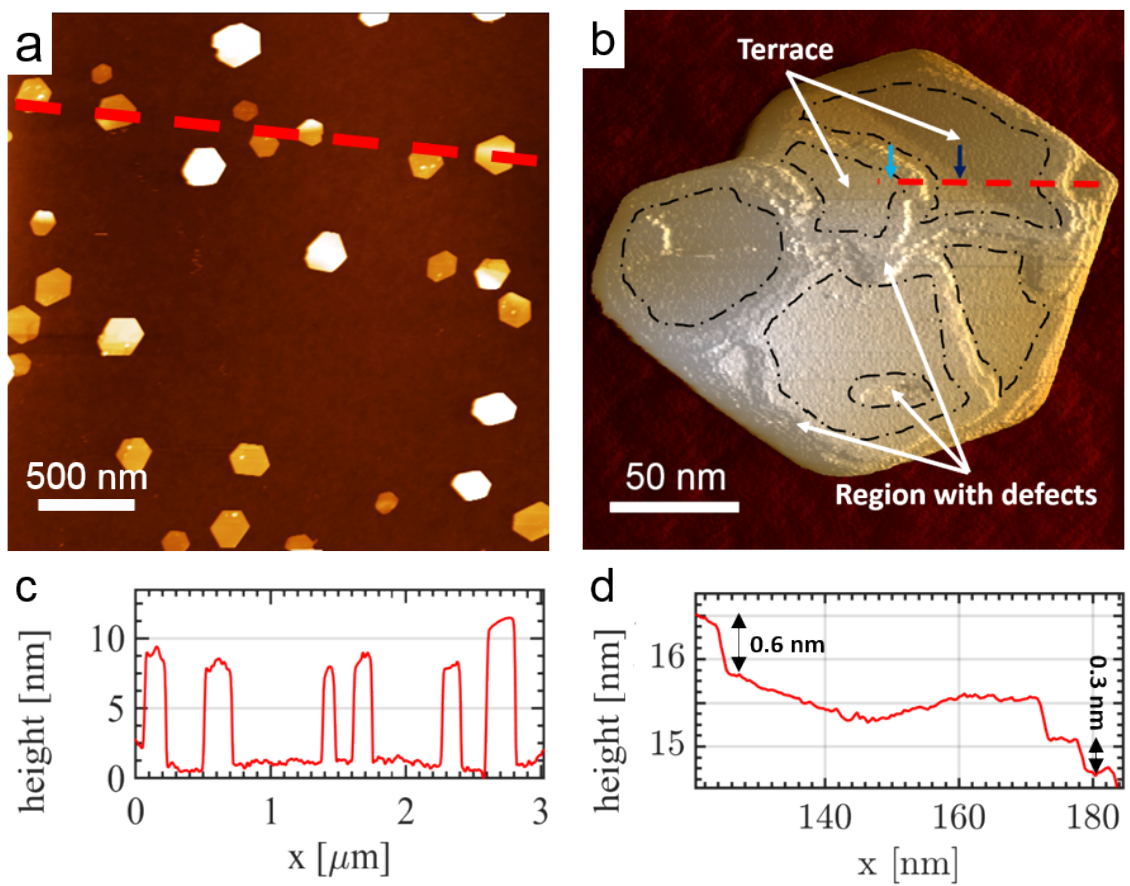

Figure 4.4: AM-AFM imaging of several gibbsite particles adsorbed on silica in a $10 \mathrm{mM}$ $\mathrm{NaCl}$ solution at $\mathrm{pH}$ 6: (a) Topography image and (c) height profile corresponding to the scan lines shown in (a). (b) Topography image of a single gibbsite particle, showing the presence of smooth terraces and regions with defects on the gibbsite basal plane and (d) corresponding scan line showing the height distribution of the basal plane of the particles and the presence of monatomic and 2-aluminum-atomic-layer steps $(\approx 0.6 \mathrm{~nm})$. Tip parameters: $Q=2.8, \omega_{0}=21.6 \mathrm{kHz}, k=0.64 \mathrm{~N} \mathrm{~m}^{-1}, R \approx 5 \mathrm{~nm}$. 

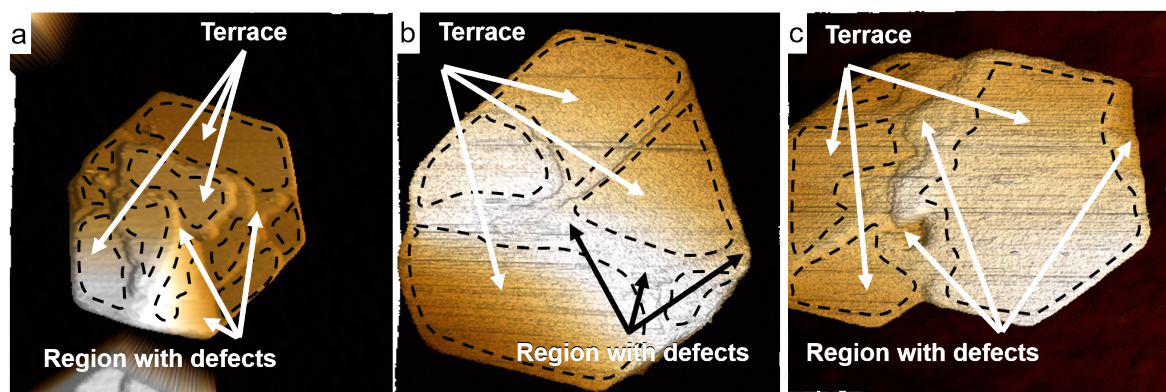

Figure 4.5: Topographic images of single gibbsite particles on silica in $10 \mathrm{mM} \mathrm{NaCl} \mathrm{pH}$ 6, showing the presence of smooth terraces and regions with defects. (a) Particle used in the charge maps found in figure 4.12. (b) Particle used in the charge maps found in figure 4.11 .

tive interaction forces, respectively. The forces are probed with a negatively charged silica tip, which implies that the gibbsite surface is positively charged and the silica surface negatively. Moreover, the gibbsite particle displays, compared to the silica substrate, more lateral variation in the interaction force. Particularly, near regions where the gibbsite surface shows topographic deviations, the forces are less attractive. This can be seen in figure $4.6 \mathrm{~b}$, which shows some of the force-distance curves for these areas. The repulsive force curves, which were measured at a number of sites on the silica substrate (red in figure $4.6 \mathrm{~b}$ ), collapse nicely. The interaction curves measured above the gibbsite particle show more variation. Attractive forces of almost equal strength can be found on the flat terraces (blue), while near the regions with defects weaker forces with a large spread in strength (green) are observed (figure $4.6 \mathrm{~b}$ ). In these experiments, tips with a radius of $5 \mathrm{~nm}$ have been used. This implies a lateral averaging of the local interaction force over an area of approximately two hundred surface unit cells.

\subsubsection{Surface charge maps}

From the recorded force-distance curves the surface charge density and its spatial distribution is extracted, using DLVO theory including charge regulation on tip and substrate. The resulting surface charge maps of the same gibbsite particle on silica in 

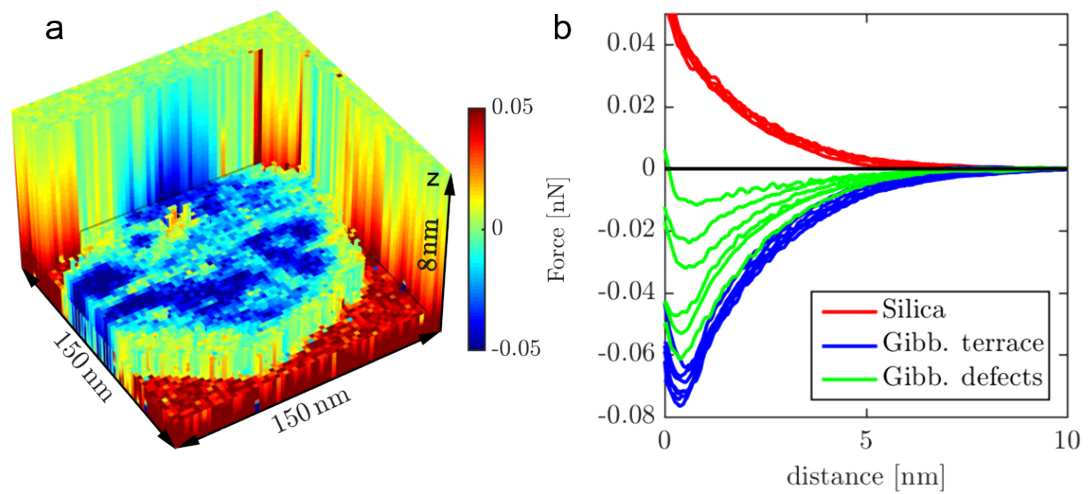

Figure 4.6: (a) A force versus distance volume plot of a gibbsite particle on silica in a $10 \mathrm{mM} \mathrm{NaCl}$ at $\mathrm{pH} 9$ solution obtained by measuring 64 by 64 approaches. The color indicates the nature of the silica tip-sample interaction. Red indicates a repulsive interaction, whereas blue indicates an attractive interaction. The silica offers a homogeneous background marked by the homogeneous red color. The gibbsite shows a much higher degree of heterogeneity. (b) $2 \mathrm{D}$ plot of selected approaches located on the gibbsite basal plane (blue), gibbsite regions with defects (green) and silica (red) converted to force vs. distance curves.

a $10 \mathrm{mM} \mathrm{NaCl}$ solution are shown in figure $4.7 \mathrm{a}$ for $\mathrm{pH} 6$ and in $4.7 \mathrm{~b}$ for $\mathrm{pH} 9$.

Effective surface charge maps recorded on other gibbsite particles and in a broader range of fluid compositions ( $\mathrm{pH}$ and $\mathrm{NaCl}$ concentration) are shown in figures 4.11 and 4.12. These maps have a coarser lateral resolution, due to a larger AFM tip and fewer points per map. They show that the silica substrate is negatively charged, while the basal plane of the gibbsite particle is positively charged. The charge distribution on the silica substrate is rather homogeneous, while, especially at $\mathrm{pH} 9$ the charge distribution on the basal plane of the gibbsite particle is more heterogeneous (Figure $4.7 \mathrm{~b}$ ). At this $\mathrm{pH}$ regions with a reduced positive charge appear. These regions correlate well with regions with a high defect density in figure $4.4 \mathrm{~b}$. When the $\mathrm{pH}$ is increased from 6 to 9 , the surface charge density in the regions with defects decreases from $0.05 \mathrm{e} / \mathrm{nm}^{2}$ to $0.02 \mathrm{e} / \mathrm{nm}^{2}$. On terraces, the charge density decreases from $0.06 \mathrm{e} / \mathrm{nm}^{2}$ to $0.05 \mathrm{e} / \mathrm{nm}^{2}$. Moreover, near the rim of the particle, where the 

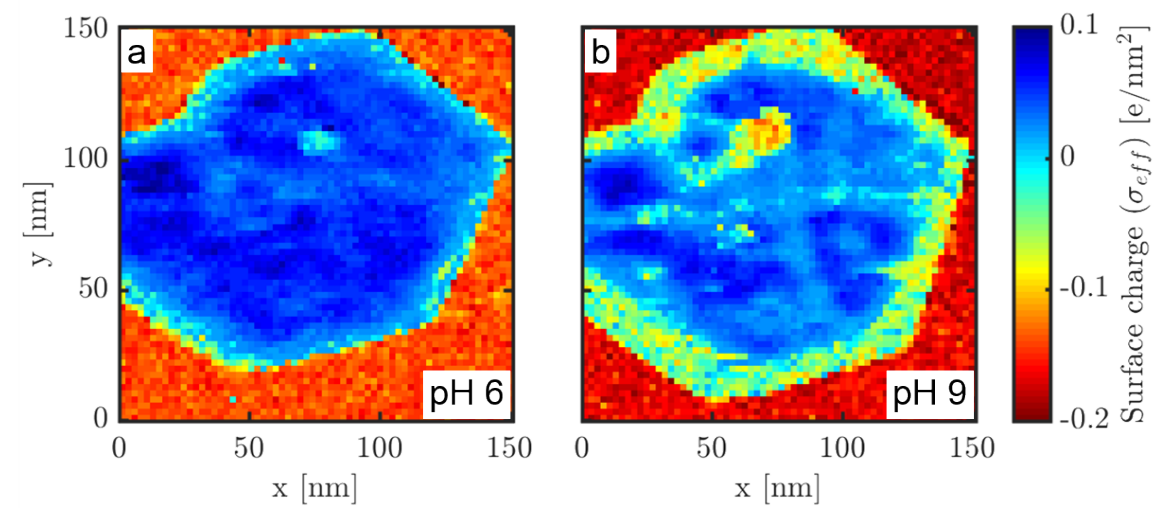

Figure 4.7: (a) The effective surface charge distribution on silica and a single gibbsite particle in $a 10 \mathrm{mM} \mathrm{NaCl} \mathrm{a)} \mathrm{pH} 5.8$ and b) $\mathrm{pH} 9.4$ solutions. The color indicates the nature of the charge. Red is the negative charge, whereas blue is the positive charge. The charge on silica is homogeneous, and heterogeneous for gibbsite. The regions of reduced charge correlate with the terrace steps and defects in figure $4.4 \mathrm{~b}$. The pixel size for this experiment is $2.3 \mathrm{~nm} / \mathrm{px}$.

density of defects is higher, the surface charge is negative $\left(-0.06 \mathrm{e} / \mathrm{nm}^{2}\right)$ at $\mathrm{pH} 9$. This is consistent with the repulsive force observed in the $3 \mathrm{D}$ force map near the rim of the gibbsite particle (Figure 4.6a). The terrace regions with a smooth topography and those with an irregular topography respond differently to changes in electrolyte composition ( $\mathrm{pH}$ and salt concentration).

This is a clear indication that the observed surface charge heterogeneity results from differences in local surface chemistry, and is not a measuring artifact caused by irregularities in the surface topography. The large blob visible in the middle top part of the particle in figure 4.7 is contamination that is also seen in the tapping mode images. The negative charge observed when the tip moves from the silica substrate to basal plane of the gibbsite particle (figure 4.8, or backward, can be attributed to 3 possible causes: i) The averaging effect due to the finite size of the AFM probe. At some regions, the cantilever tip experiences a competition of repulsive and attractive electrostatic interactions of the silica and gibbsite surface. ii) An artifact due to the steep topographic step from the silica substrate to the upper side of the gibbsite par- 

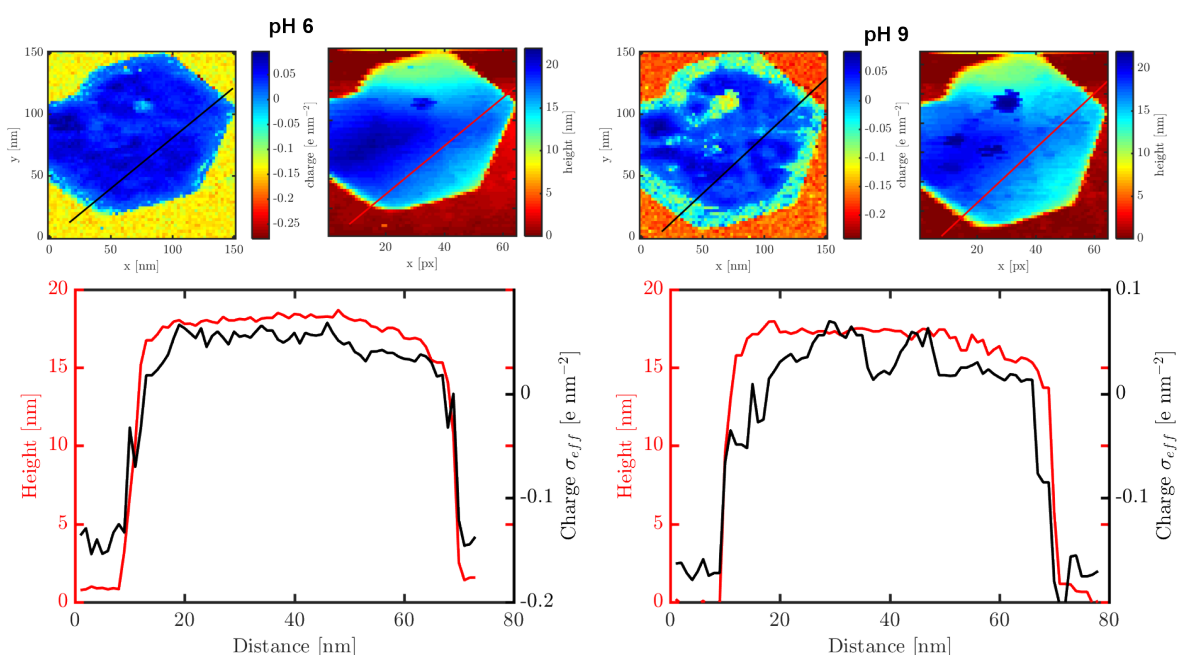

Figure 4.8: Effective surface charge maps, topography and line sections at $\mathrm{pH} 6$ and $\mathrm{pH} 9$. At $\mathrm{pH} 6$ and $\mathrm{pH}$ 9, the surface charge characteristics (in black) on gibbsite do not follow the surface roughness (in red). The pixel size is $2.3 \mathrm{~nm} / \mathrm{px}$. (Tip parameters in liquid: $\left.Q=2.8, \omega_{d}=21.6 \mathrm{kHz}, k=0.64 \mathrm{~N} \mathrm{~m}^{-1}, R \approx 5 \mathrm{~nm}\right)$.

ticle. iii) The surface chemistry at the edge of the particle, which differs from that at the central region of the particle. Although we cannot completely rule out any one of these three possibilities, it should be noted that the width of the region with negative charge near the edge of the gibbsite particle is about $\approx 25 \mathrm{~nm}$, while the tip radius is only $5 \mathrm{~nm}$. Thus option ii) is quite unlikely. At approximately 10 to $15 \mathrm{~nm}$ away from the rim of the particle, the interaction area of the AFM tip is completely located on the gibbsite platelet. Moreover, our raw data show that the tip-sample force decreases upon approaching these regions, suggesting a reduced charge density here. Therefore, we attribute the negative charge near the rim to a different surface chemistry.

The measured charge densities on defect locations suffer from convolution errors due to the finite tip-substrate contact area of about $100 \mathrm{~nm}^{2}$. The charge maps in figure 4.7 are recorded using a cantilever with a tip radius of approximately $5 \mathrm{~nm}$, which means that the tip diameter is about 4 to 5 pixels in this figure. To estimate the true local charge density from the measured reduced attractions, we have de- 

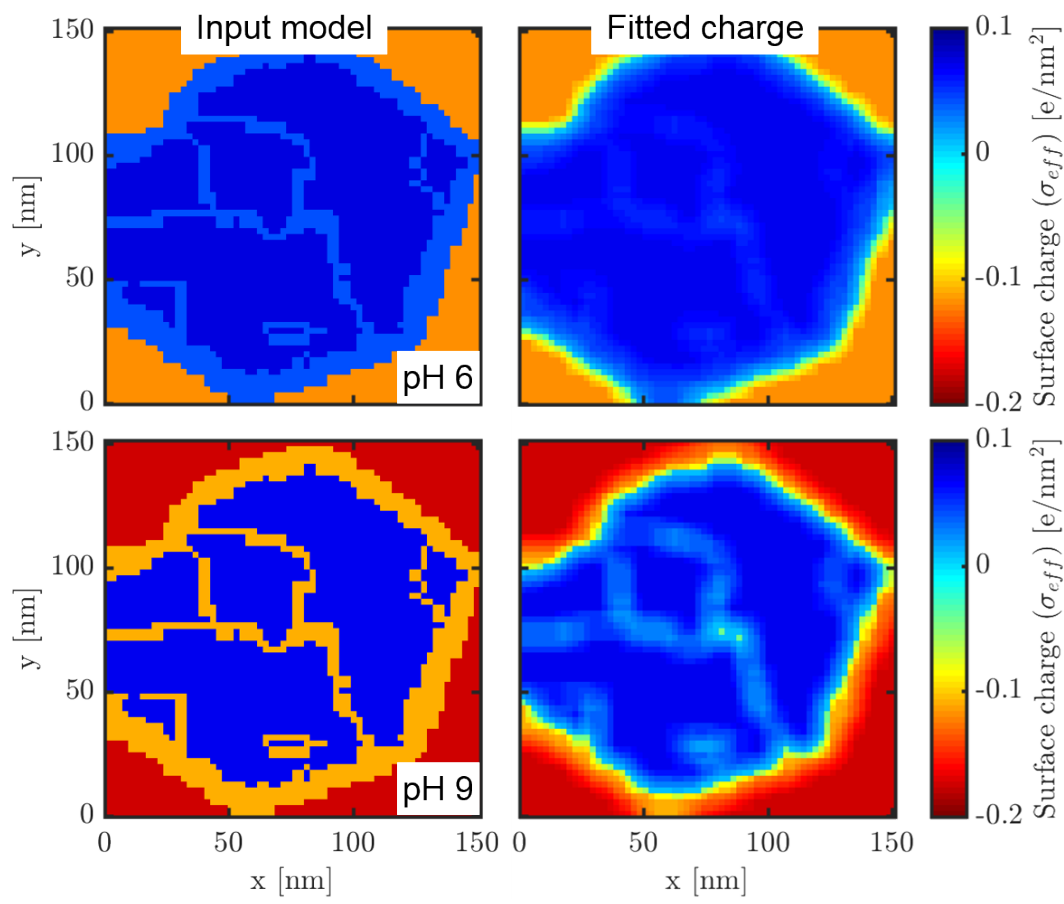

Figure 4.9: Deconvolution model in order to recover localized surface charge information. The surface heterogeneity of the model is based on the topographic features from the experiment in figure 4.4 b and 4.7. Every pixel in the model is assigned a surface complexation model. For gibbsite two different complexation models and $p K$ pairs were used, one for the flat terraces and the other for the regions with defects. The $p K$ pairs used for silica $\left(p K_{H}=4.5\right.$ and $\left.p K_{C}=2.1\right)$, gibbsite terrace sites $\left(p K_{1}=3.8\right.$ and $\left.p K_{2}=3\right)$, gibbsite region with defects $\left(p K_{1}=4\right.$ and $\left.p K_{2}=2.5\right)$. For each pixel, the interaction force is calculated, and then an average of the force across the tip area ( $5 x 5$ pixels) is taken. The average force is used to fit the charge, which results in the right column. The model is able to reproduce the heterogeneity observed in the experiment. Note that in order to reproduce the experimental charge values at $\mathrm{pH}$ 9, a negative charge has to be assigned to the regions with defects. 
veloped a numerical scheme with which we calculate the convoluted charge density from an assumed charge density distribution over the particle. For the calculations, we use a grid size $(150 \mathrm{~nm} \times 150 \mathrm{~nm})$ and grid-texture (64 pixels x 64 pixels) that matches the settings used in the experiments. For every pixel on the grid, a particular surface complexation model is assumed. For pixels on the substrate, we assign a silica complexation model with chemical reaction: $\equiv \mathrm{SiOH} \rightleftharpoons \equiv \mathrm{SiO}^{-}+\mathrm{H}^{+}$ and $\equiv \mathrm{SiONa} \rightleftharpoons \equiv \mathrm{SiO}^{-}+\mathrm{Na}^{+}$with corresponding $\mathrm{pK}$ values $\mathrm{pK}$ $\mathrm{pK}_{\mathrm{C}}=2.1$. The same complexation equations are used for the model tip, which is a square $124 \mathrm{~nm}^{2}$ large tip. On the nanoparticle, we assume two types of regions: one that resembles the flat terraces and the other corresponding to the defect positions. The defects sites are located at the rim of the particle and in the central region of the particle, similar to the location of defects in the topography image of figure $4.4 \mathrm{~b}$. For these regions, we assume the following complexation model: $\equiv \mathrm{Al}_{2} \mathrm{OH}_{2}{ }^{+} \rightleftharpoons$ $\equiv \mathrm{Al}_{2} \mathrm{OH}+\mathrm{H}^{+}$and $\equiv \mathrm{Al}_{2} \mathrm{OH} \rightleftharpoons \mathrm{Al}_{2} \mathrm{O}^{-}+\mathrm{H}^{+}$with $\mathrm{pK}_{1}=3.8$ and $\mathrm{pK}_{2}=3$ (for terrace sites) and $\mathrm{pK}_{1}=4$ and $\mathrm{pK}_{2}=2.5$ (on the defects). The size of the tip, the position of defects, and complexation models are chosen such that the numerically generated convoluted charged maps resemble the experimentally measured maps as good as possible. Using the above described complexation models, we calculate the effective surface charge and tip-sample interaction force (data not shown) for each individual pixel at $\mathrm{pH} 6$ and $\mathrm{pH} 9$ in $10 \mathrm{mM} \mathrm{NaCl}$. The corresponding charge densities are shown in figure 4.9, left hand images. Then, in the convolution step, we take the average of the forces across the contact area between tip and surface. Subsequently, this average interaction force was used to fit the surface charge for every pixel. The convoluted charge calculation results are shown in the right hand images of figure 4.9. The simulation results reflect the experimental results well, in terms of charge heterogeneity, the absolute values of the surface charge in regions with defects and terraces, and even the charge inversion at the rim of the particle. In experimentally measured charged maps, at $\mathrm{pH} 6$ the convoluted image shows little charge difference between regions with defects and terraces. The difference in protonation level of the defect sites and the terraces is almost negligible at $\mathrm{pH} 6$. For $\mathrm{pH} 9$, the difference is more pronounced and in good agreement with the experimentally measured values. This pronounced difference is caused by a negative deconvoluted charge value $(\approx$ $\left.-0.1 \mathrm{e} / \mathrm{nm}^{2}\right)$ at the defect sites on the basal plane. This value is close to the charge measured at the rim of the particle $\left(-0.06 \mathrm{e} / \mathrm{nm}^{2}\right)$. This suggests that the proton donor-acceptor reactions and functional groups in these regions are similar. More- 
over, our analysis shows that the observation of such small features is only possible with AFM tips with radii as small as $5 \mathrm{~nm}$. As shown in figures 4.11 and 4.12, for tips with a larger contact area $\left(\geq 150 \mathrm{~nm}^{2}\right)$, the charge heterogeneity across these regions with defects, as observed in the topographic images, is completely averaged out for $\mathrm{pH}$ conditions. Only charge reversal at the rim of the particle at $\mathrm{pH} 9$ is visible. Therefore, it is not surprising that Gan et al. [6] did not observe any charge heterogeneity and/or negative charge on the basal plane of the particle, because they used tips with a diameter as large as $5.08 \mu \mathrm{m}$. However, in the topographic image that was probed with a $7 \mathrm{~nm}$ tip, steps and imperfections can be distinguished. From the data in figures 4.10, 4.11 and 4.12, we can extract average charge density values on the gibbsite basal plane as a function of $\mathrm{pH}$ and $\mathrm{NaCl}$ concentration. These are presented in figure 4.10. The charge data from the rim of the gibbsite nanoparticles are excluded from this averaging.

Two significant features of the charging behavior of the gibbsite basal plane can be observed. First, at $\mathrm{pH} 4$ or higher, the surface is positively charged; the charge decreases with increasing $\mathrm{pH}$ from 4 to 9 ; therefore, the PZC of the gibbsite basal plane lies above $\mathrm{pH}$ 9. On the other hand, under all investigated conditions the silica is negatively charged, and its charge increases with increasing $\mathrm{pH}$, because the fraction of deprotonated $\mathrm{SiO}^{-}$groups monotonically increases with increasing $\mathrm{pH}$. This agrees quantitatively with the values reported in literature for oxidized silicon wafers $[8,26,41]$. Second, in the presence of $\mathrm{NaCl}$ salt, the effective surface charge on gibbsite and silica increases with increasing salinity. The enhanced screening of the electric field with the increasing salt concentration reduces the energetic cost for the system to deprotonate (for silica) or protonate (for gibbsite).

\subsection{Discussion}

The above AFM analysis shows that the gibbsite basal plane (0o1) is chemically active, and has a net positive charge that varies with $\mathrm{pH}$ and $\mathrm{NaCl}$ concentration. Our observations contradict the conclusion of earlier titration data, where the $\mathrm{pH}$ responsive sites and the resulting charge densities were attributed to the edges only. But they support the more recent work of Gan et al. [6], who used colloidal probe AFM force spectroscopy to observe the $\mathrm{pH}$ dependency of the gibbsite basal plane. A 

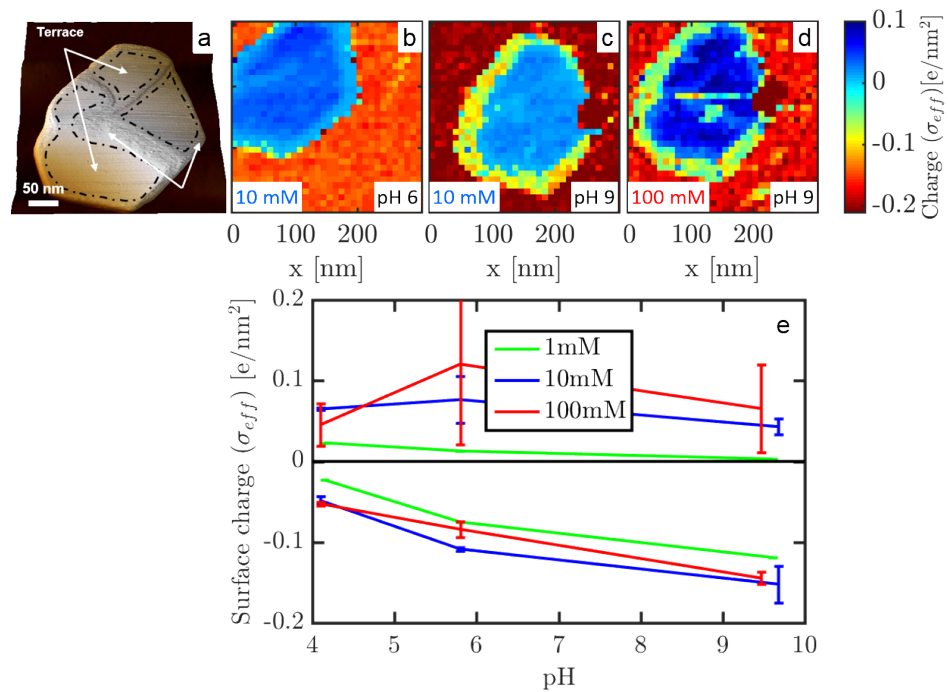

Figure 4.10: (a) Topography image of a single gibbsite particle on silica, showing the presence of smooth terraces and regions with defects on the gibbsite basal plane. The effective surface charge calculated for a single gibbsite particle on silica in (b) $10 \mathrm{mM} \mathrm{NaCl} \mathrm{pH} \mathrm{6,}$ (c) $10 \mathrm{mM} \mathrm{NaCl} \mathrm{pH} 9$ and (c) $100 \mathrm{mM} \mathrm{NaCl} p H 9$ solution. More surface charge maps for additional conditions can be found in figure 4.11 and 4.12. (e) The surface charge versus $\mathrm{pH}$ for a selected region on gibbsite (on a smooth terrace) and silica. 

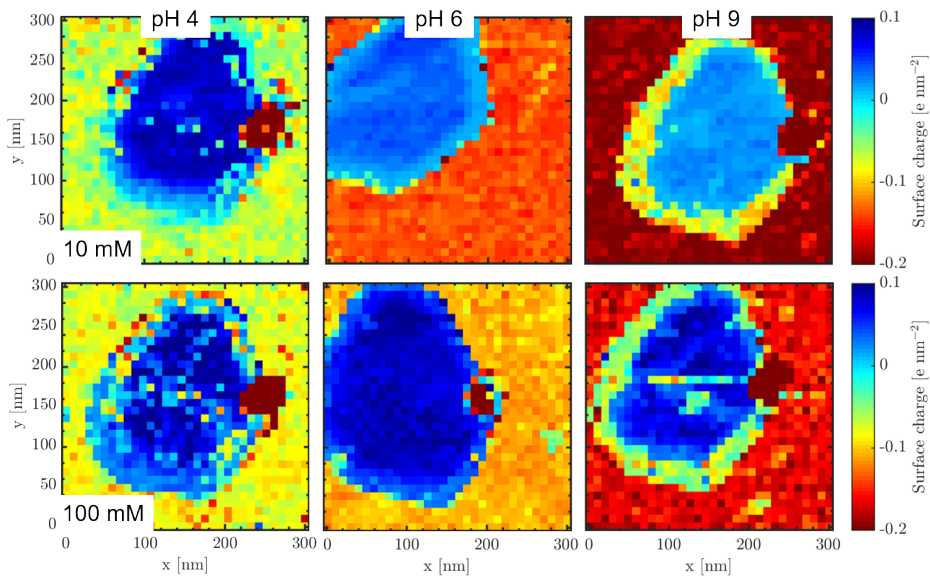

Figure 4.11: Effective surface charge maps of a single gibbsite particle on silica (Figure $4.5 \mathrm{~b}$ ) in several solutions ( 10 and $100 \mathrm{mM} \mathrm{NaCl}$ at $\mathrm{pH} 4,6$ and 9). Red and blue are negative and positive charge respectively. With increasing $\mathrm{pH}$, the negative charge on silica increases, , while the charge on gibbsite decreases. At $\mathrm{pH} 9$, charge reversal is observed at the rim of the gibbsite particle. The pixel size is $9.4 \mathrm{~nm} / \mathrm{px}$. (Tip parameters in liquid: $\mathrm{Q}=3.5, \omega_{\infty}=48.6 \mathrm{kHz}, \omega_{d}=49.4 \mathrm{kHz}, A_{\mathrm{o}}=0.55 \mathrm{~nm}, k=0.65 \mathrm{~N} \mathrm{~m}^{-1}, R \approx$ $9 \mathrm{~nm}$ ) 


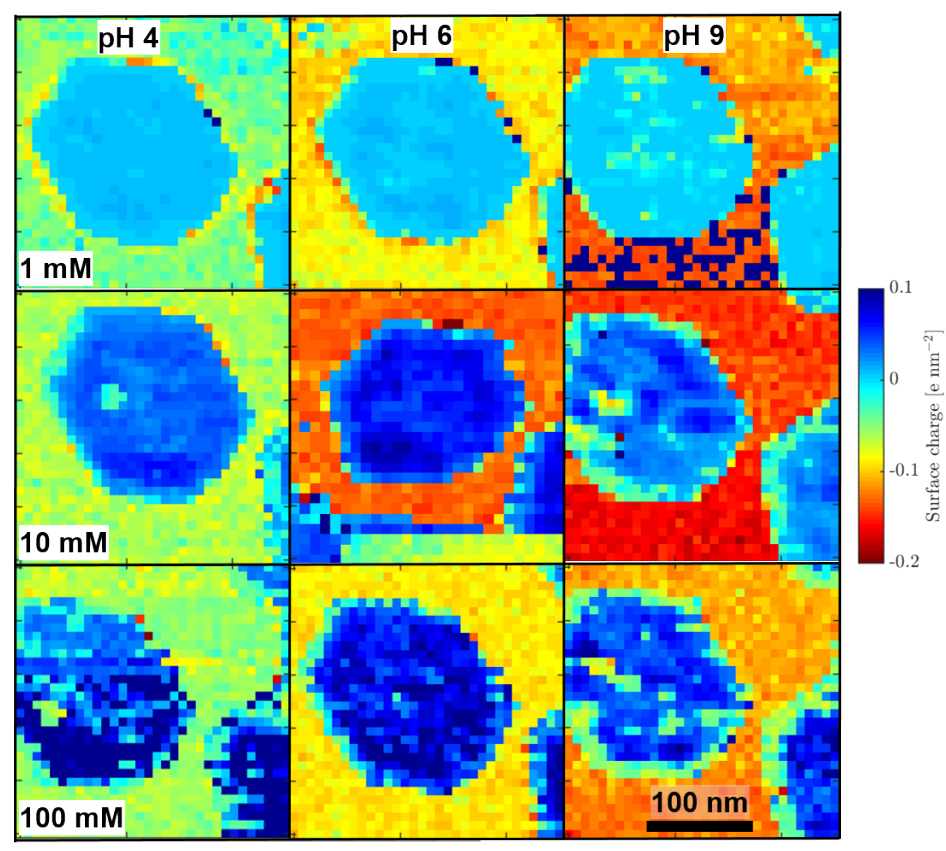

Figure 4.12: Effective surface charge maps of a single gibbsite particle on silica (Figure 4.5 a) in several solutions (1, 10 and $100 \mathrm{mM} \mathrm{NaCl}$ at $\mathrm{pH} 4,6$ and 9). Red and blue are negative and positive charge respectively. With increasing $p H$, silica gets more negatively charged, while gibbsite gets less positively charged. At $\mathrm{pH}$ 9, charge reversal is observed at the rim of the gibbsite particle. The pixel size is $7.8 \mathrm{~nm} / \mathrm{px}$. (Tip parameters in liquid: $\left.Q=2.6, \omega_{\infty}=17.8 \mathrm{kHz}, \omega_{d}=16.5 \mathrm{kHz}, A_{\circ}=2.0 \mathrm{~nm}, k=0.65 \mathrm{~N} \mathrm{~m}^{-1}, R \approx 7 \mathrm{~nm}\right)$ 
similar $\mathrm{pH}$ dependence was observed on the gibbsite facet of kaolinite particles by Gupta et al. [53]. In their experiments, the surface charge changed from $0.012 \mathrm{e} / \mathrm{nm}^{2}$ to $-0.037 \mathrm{e} / \mathrm{nm}^{2}$ when the $\mathrm{pH}$ was increased from 6 to 8 . We do not observe charge reversal for the smooth terraces on the gibbsite basal plane, but we do observe reversal at the defect sites. This can be due to protonation/deprotonation of the doubly coordinated hydroxyl groups on the basal plane, as suggested by Bickmore et al. [32] and Jodin et al. [9]. Using colloidal probe force spectroscopy, Gan et al. [6] and Gupta et al. [53] , could determine the average charge density on the basal plane. In this study, we are able, due to the use of sharp tips, to discriminate between the charge density at the defect sites and that on smooth terraces. The observed surface charge heterogeneity correlates with the presence of topographic defects such as steps. Moreover, comparing the charge maps acquired for different $\mathrm{pH}$ and $\mathrm{NaCl}$ concentration values show that defects are chemically more active than the smooth regions, because the regions with defects respond stronger to $\mathrm{pH}$ variations. The smooth parts of the surface carry a charge density that decreases slightly with increasing $\mathrm{pH}$ (from $0.06 \mathrm{e} / \mathrm{nm}^{2}$ at $\mathrm{pH} 6$ to $\approx 0.05 \mathrm{e} / \mathrm{nm}^{2}$, at $\mathrm{pH} 9$ ), while in regions with defects the charge decreases more strongly with increasing $\mathrm{pH}$ (from $0.05 \mathrm{e} / \mathrm{nm}^{2}$ at $\mathrm{pH} 6$ to a value between -0.06 and $0.02 \mathrm{e} / \mathrm{nm}^{2}$ for $\mathrm{pH} 9$ ). Moreover, the absolute value of the charges is rather small. An effective surface charge value of $0.05 \mathrm{e} / \mathrm{nm}^{2}$ would correspond to only 1 positive charge in about 40 unit cells. In other words, the average distance between charged sites on the surface is of the order of a few nanometers, which is comparable to the radius of the AFM tips that are typically used for highresolution AFM imaging. From this perspective, it is not surprising that sharp tips hardly feel the presence of such small degrees of surface charge. Therefore, almost every unit cell on the basal plane is electrically neutral. Hence, the small effective surface charge on the smooth terraces can result from protonation/ deprotonation of surface groups associated with smaller defects as suggested by Hiemstra et al. [13,28]. However, especially at $\mathrm{pH}$, the chemical behavior at larger defects, such as terrace steps, induces negative charge densities, while these small defects should induce a positive charge density, if they are responsible for the charge behavior of the smooth parts of the basal plane. In previous studies [27] on atomic resolution imaging, we observed various small defects on the basal planes of clay minerals, including vacancies of one or few atoms and ad-atoms. The effective surface charge on the basal plane can also originate from more complex processes, such as specific ion adsorption, like $\mathrm{Na}^{+}$or $\mathrm{Ca}^{2+}$ adsorption, on neutral sites on the gibbsite basal plane, as assumed by 
Rosenquist et al. [12] and experimentally shown by Siretanu et al. [8]. Yet, from force spectroscopy measurements, the origin of the charge near defects, nor the charge on the smooth terraces on the basal plane of the gibbsite particle cannot be identified. In order to confirm which surface reactions occur at the gibbsite basal plane, more spectroscopic evidence is needed, e.g. from x-ray absorption spectroscopy. As AFM force spectroscopy only reveals the (diffusive layer contribution to) the surface charge it will never be able to discriminate between different surface complexation models associated with local surface chemistry. However, the impact of surface defects on the properties of nanoparticles in water is until now only addressed in a few publications. The data presented in this paper are the first of its kind, showing the impact of surface defects on the surface charge density, which is not accessible with common macroscopic measurements. Better understanding of the role of surface defects and their chemical properties will have a great impact on the understanding of various processes, such as ion adsorption, wettability alteration, the stability of colloidal systems and adsorption/desorption behavior of oil components on clays and rock surfaces.

\subsection{Conclusion}

In the present work, we characterize the effective surface charge distribution on the basal plane of gibbsite particles with high lateral resolution atomic force microscopy. Using force spectroscopy, we extract the diffuse layer charge of the gibbsite (oo 1) surface using DLVO theory with charge regulation. We have found that the basal plane of gibbsite does carry a finite charge. This charge density is highly inhomogeneous and $\mathrm{pH}$ dependent. Charge maps with a lateral resolution of $5 \mathrm{~nm}$ show that the effective surface charge distribution on the basal plane correlates with the topographic defects, such as terrace steps on that plane. Furthermore, we found that the defects on the basal plane are more responsive to $\mathrm{pH}$ changes than the smooth terraces. At $\mathrm{pH}$ 9 the defect sites carry a negative charge density of approximately $-0.1 \mathrm{e} / \mathrm{nm}^{2}$ while the smooth terraces carry a positive charge density of approximately $0.05 \mathrm{e} / \mathrm{nm}^{2}$. However, to test microscopic surface complexation models beyond the resulting surface charges, one requires a more detailed experimental characterization of the surface groups present at the basal plane. More generally, this study demonstrates the capabilities of atomic force spectroscopy to study local surface charge properties of 
nanoparticles with nanoscale lateral resolution. So far, we have restricted our study to the influence of rather large surface defects. However, this work can be considered as a first step towards the investigation of local variations in the surface properties of nanoparticles due to topographic defects with atomic resolution.

\section{Bibliography}

[1] F. Mugele, B. Bera, A. Cavalli, I. Siretanu, A. Maestro, M. Duits, M. CohenStuart, D. van den Ende, I. Stocker, and I. Collins, "Ion adsorption-induced wetting transition in oil-water-mineral systems," Sci Rep, vol. 5, p. 105 19, 2015.

[2] P. F. Mugele, I. Sîretanu, N. Kumar, B. Bera, L. Wang, M. Maestro, D. M. Duits, D. H. v. d. Ende, and I. Collins, "Charge control and wettability alteration at solid-liquid interfaces," 2014.

[3] F. Mugele, I. Siretanu, N. Kumar, B. Bera, L. Wang, R. de Ruiter, A. Maestro, M. Duits, D. van den Ende, and I. Collins, "Insights from ion adsorption and contact-angle alteration at mineral surfaces for low-salinity waterflooding," SPE Journal, 2016.

[4] C. A. J. Appelo and D. Postma, Geochemistry, groundwater and pollution. CRC press, 2004 .

[5] G. V. Franks and Y. Gan, "Charging behavior at the alumina-water interface and implications for ceramic processing," Journal of the American Ceramic Society, vol. 90 , no. 11 , pp. $3373-3388,2007$.

[6] Y. Gan and G. V. Franks, "Charging behavior of the gibbsite basal (oo1) surface in nacl solution investigated by afm colloidal probe technique," Langmuir, vol. 22, no. 14, pp. 6087-6092, 2006.

[7] F. Adekola, M. Fedoroff, H. Geckeis, T. Kupcik, G. Lefevre, J. Lutzenkirchen, M. Plaschke, T. Preocanin, T. Rabung, and D. Schild, "Characterization of acid-base properties of two gibbsite samples in the context of literature results," Journal of Colloid and Interface Science, vol. 354, no. 1, pp. 306-317, 2011. 
[8] I. Siretanu, D. Ebeling, M. P. Andersson, S. L. Stipp, A. Philipse, M. C. Stuart, D. van den Ende, and F. Mugele, "Direct observation of ionic structure at solidliquid interfaces: a deep look into the stern layer," Sci Rep, vol. 4, p. 4956, 2014.

[9] M. C. Jodin, F. Gaboriaud, and B. Humbert, "Limitations of potentiometric studies to determine the surface charge of gibbsite gamma-al(oh)(3) particles," Journal of Colloid and Interface Science, vol. 287, no. 2, pp. 581-591, 2005.

[10] J. Lutzenkirchen, Surface complexation modelling, vol. 11. Academic press, 2006.

[1 1 E. Tombacz and M. Szekeres, "Interfacial acid-base reactions of aluminum oxide dispersed in aqueous electrolyte solutions. 1. potentiometric study on the effect of impurity and dissolution of solid phase," Langmuir, vol. 17, no. 5, pp. 1411-1419, 2001.

[12] J. Rosenquist, P. Persson, and S. Sjoberg, "Protonation and charging of nanosized gibbsite (alpha-al(oh)(3)) particles in aqueous suspension," Langmuir, vol. 18 , no. 12, pp. 4598-4604, 2002.

[13] T. Hiemstra, H. Yong, and W. H. Van Riemsdijk, "Interfacial charging phenomena of aluminum (hydr)oxides," Langmuir, vol. 15, no. 18, pp. 5942-5955, 1999.

[14] R. Weerasooriya, B. Dharmasena, and D. Aluthpatabendi, "Copper-gibbsite interactions: an application of 1-pk surface complexation model," Colloids and Surfaces a-Physicochemical and Engineering Aspects, vol. 170, no. 1, pp. 65-77, 2000.

[15] J. Lutzenkirchen, A. Abdelmonem, R. Weerasooriya, F. Heberling, V. Metz, and R. Marsac, "Adsorption of dissolved aluminum on sapphire-c and kaolinite: implications for points of zero charge of clay minerals," Geochem Trans, vol. 15 , p. 9, 2014 .

[16] D. A. Palmer and D. J. Wesolowski, "Aluminum speciation and equilibria in aqueous-solution .2. the solubility of gibbsite in acidic sodium-chloride solutions from 30-degrees-c to 70-degrees-c," Geochimica Et Cosmochimica Acta, vol. 56, no. 3, pp. 1093-1111, 1992. 
[17] A. M. Wierenga, T. A. J. Lenstra, and A. P. Philipse, "Aqueous dispersions of colloidal gibbsite platelets: synthesis, characterisation and intrinsic viscosity measurements," Colloids and Surfaces a-Physicochemical and Engineering Aspects, vol. 134, no. 3, pp. 359-371, 1998.

[18] X. Yang, Z. Sun, D. Wang, and W. Forsling, "Surface acid-base properties and hydration/dehydration mechanisms of aluminum (hydr)oxides," J Colloid Interface Sci, vol. 308, no. 2, pp. 395-404, 2007.

[19] F. Gaboriaud and J. Ehrhardt, "Effects of different crystal faces on the surface charge of colloidal goethite (alpha-feooh) particles: An experimental and modeling study," Geochimica Et Cosmochimica Acta, vol. 67, no. 5, pp. 967-983, 2003.

[20] C. P. Huang and W. Stumm, "Specific adsorption of cations on hydrous gammaal203," Journal of Colloid and Interface Science, vol. 43, no. 2, pp. 409-420, 1973.

[21] J. A. Davis, R. O. James, and J. O. Leckie, "Surface ionization and complexation at the oxide/water interface: I. computation of electrical double layer properties in simple electrolytes," Journal of colloid and interface science, vol. 63, no. 3 , pp. 480-499, 1978.

[22] G. A. Parks, "The isoelectric points of solid oxides, solid hydroxides, and aqueous hydroxo complex systems," Chemical Reviews, vol. 65, no. 2, pp. 177-198, 1965 .

[23] G. V. Franks and L. Meagher, "The isoelectric points of sapphire crystals and alpha-alumina powder," Colloids and Surfaces A: Physicochemical and Engineering Aspects, vol. 214, no. 1, pp. 99-1 10, 2003.

[24] M. Kosmulski, "The ph-dependent surface charging and the points of zero charge," Journal of Colloid and Interface Science, vol. 253, no. 1, pp. 77-87, 2002.

[25] X. Liu, J. Cheng, M. Sprik, X. Lu, and R. Wang, "Understanding surface acidity of gibbsite with first principles molecular dynamics simulations," Geochimica et Cosmochimica Acta, vol. 120, pp. 487-495, 2013.

[26] N. Kumar, C. Zhao, A. Klaassen, D. van den Ende, F. Mugele, and I. Siretanu, "Characterization of the surface charge distribution on kaolinite particles using 
high resolution atomic force microscopy," Geochimica et Cosmochimica Acta, vol. 175 , pp. 100-112, 2016.

[27] I. Siretanu, D. van den Ende, and F. Mugele, "Atomic structure and surface defects at mineral-water interfaces probed by in situ atomic force microscopy," Nanoscale, vol. 8, no. 15, pp. 8220-7, 2016.

[28] T. Hiemstra and W. H. Van Riemsdijk, "A surface structural approach to ion adsorption: The charge distribution (cd) model," Journal of Colloid and Interface Science, vol. 179, no. 2, pp. 488-508, 1996.

[29] T. Hiemstra, P. Venema, and W. Van Riemsdijk, "Intrinsic proton affinity of reactive surface groups of metal (hydr) oxides: The bond valence principle," Journal of colloid and interface science, vol. 184, no. 2, pp. 680-692, 1996.

[30] T. Hiemstra, W. Van Riemsdijk, and G. Bolt, "Multisite proton adsorption modeling at the solid/solution interface of (hydr) oxides: A new approach: I. model description and evaluation of intrinsic reaction constants," Journal of colloid and interface science, vol. 133, no. 1, pp. 91-104, 1989.

[31] A. K. Karamalidis and D. A. Dzombak, Surface complexation modeling: gibbsite. John Wiley \& Sons, 2011.

[32] B. R. Bickmore, K. M. Rosso, C. J. Tadanier, E. J. Bylaska, and D. Doud, "Bondvalence methods for $\mathrm{pk}(\mathrm{a})$ prediction. ii. bond-valence, electrostatic, molecular geometry, and solvation effects," Geochimica Et Cosmochimica Acta, vol. 70, no. 16 , pp. 4057-4071, 2006.

[33] B. R. Bickmore, C. J. Tadanier, K. M. Rosso, W. D. Monn, and D. L. Eggett, "Bond-valence methods for $\mathrm{pk}(\mathrm{a})$ prediction: critical reanalysis and a new approach," Geochimica Et Cosmochimica Acta, vol. 68, no. 9, pp. 202 5-2042, 2004.

[34] C. L. Zhao, D. Ebeling, I. Siretanu, D. van den Ende, and F. Mugele, "Extracting local surface charges and charge regulation behavior from atomic force microscopy measurements at heterogeneous solid-electrolyte interfaces," Nanoscale, vol. 7, no. 39, pp. 16298-16311, 2015. 
[35] S. H. Behrens and M. Borkovec, "Electric double layer interaction of ionizable surfaces: Charge regulation for arbitrary potentials," Journal of Chemical Physics, vol. 111 , no. 1, pp. 382-385, 1999.

[36] G. Sposito, The Environmental Chemistry of Aluminum, Second Edition. Taylor and Francis, 1995.

[37] S. Goldberg, J. A. Davis, and J. D. Hem, “The surface chemistry of aluminum oxides and hydroxides," The environmental chemistry of aluminum, pp. 271-331, 1996.

[38] R. A. Yokel, "Aluminum," Elements and Their Compounds in the Environment: Occurrence, Analysis and Biological Relevance, Second Edition, pp. 635-658, 2004.

[39] L. Sirghi, R. Szoszkiewicz, and E. Riedo, "Volume of a nanoscale water bridge," Langmuir, vol. 22, no. 3, pp. 1093-1098, 2006.

[40] A. Labuda, J. Cleveland, N. A. Geisse, M. Kocun, B. Ohler, R. Proksch, M. B. Viani, and D. Walters, "Photothermal excitation for improved cantilever drive performance in tapping mode atomic force microscopy," Microscopy and Analysis2 8, vol. 3, pp. $\mathrm{S}_{21}-\mathrm{S}_{25}, 2014$.

[41] N. Umeda, S. Ishizaki, and H. Uwai, "Scanning attractive force microscope using photothermal vibration," Journal of Vacuum Science \& Technology B, vol. 9, no. 2, pp. 1318-1322, 1991.

[42] C. A. J. Putman, K. O. Vanderwerf, B. G. Degrooth, N. F. Vanhulst, and J. Greve, "Tapping mode atomic-force microscopy in liquid," Applied Physics Letters, vol. 64 , no. 18 , pp. 2454-2456, 1994.

[43] D. Kiracofe and A. Raman, "Quantitative force and dissipation measurements in liquids using piezo-excited atomic force microscopy: a unifying theory," Nanotechnology, vol. 22, no. 48, 2011.

[44] F. Liu, C. L. Zhao, F. Mugele, and D. van den Ende, "Amplitude modulation atomic force microscopy, is acoustic driving in liquid quantitatively reliable?" Nanotechnology, vol. 26, no. 38, 2015. 
[45] S. de Beer, D. van den Ende, and F. Mugele, Small Amplitude Atomic Force Spectroscopy. 2011.

[46] F. Liu, S. de Beer, D. van den Ende, and F. Mugele, "Atomic force microscopy of confined liquids using the thermal bending fluctuations of the cantilever," Physical Review E, vol. 87, no. 6, p. 062406, 2013.

[47] B. W. Ninham and Parsegia.Va, "Electrostatic potential between sufraces bearing ionizable groups in ionic equilibrium with physiologic saline solution," Journal of Theoretical Biology, vol. 31, no. 3, p. 405, 1971.

[48] S. H. Behrens and M. Borkovec, "Electric double layer interaction of ionizable surfaces: Charge regulation for arbitrary potentials," Journal of Chemical Physics, vol. 111, no. 1, pp. 382-385, 1999.

[49] M. Dishon, O. Zohar, and U. Sivan, "From repulsion to attraction and back to repulsion: The effect of nacl, $\mathrm{kcl}$, and cscl on the force between silica surfaces in aqueous solution," Langmuir, vol. 25, no. 5, pp. 2831-2836, 2009.

[50] H. J. Butt, B. Cappella, and M. Kappl, "Force measurements with the atomic force microscope: Technique, interpretation and applications," Surface Science Reports, vol. 59, no. 1-6, pp. 1-152, 2005.

[51] B. Cappella and G. Dietler, "Force-distance curves by atomic force microscopy," Surface Science Reports, vol. 34, no. 1-3, pp. 1-+, 1999.

[52] C. Sweegers, W. J. P. van Enckevort, H. Meekes, P. Bennema, I. D. K. Hiralal, and A. Rijkeboer, "The impact of twinning on the morphology of gammaal(oh)(3) crystals," Journal of Crystal Growth, vol. 197, no. 1-2, pp. 244-253, 1999.

[53] V. Gupta and J.D. Miller, "Surface force measurements at the basal planes of ordered kaolinite particles," Journal of Colloid and Interface Science, vol. 344, no. 2, pp. $362-371,2010$. 


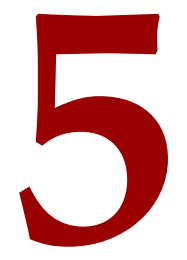

\section{Mapping electrostatic and structural hydration forces of mineral gibbsite nanoparticles in electrolyte solutions using atomic force} microscopy

THE INTERACTION OF WATER WITH MINERAL SURFACES (silica and alumina) is one of the most important chemical reactions occurring in nature. Interfacial water and hydration properties play a major role in dissolution, $\mathrm{CO}_{2}$ sequestration, precipitation and sorption processes affecting the composition and quality of natural waters. Mapping the mineral nanoparticles hydration structure is therefore essential for (geo)chemists and environmental scientists to understand the molecular processes. Here, using amplitude-modulation atomic force microscopy (AM-AFM), we map simultaneously the hydration structure (hydrations forces), effective surface charge (EDL force) and topography of single gibbsite nanoparticle on a silica sub- 
strate, upon exposure to solutions of variable $\mathrm{pH}$ and $\mathrm{NaCl}$ concentrations. The force versus distance curves measured between the AFM tip and the silica-gibbsite particle clearly show oscillatory profiles. The oscillations peak spacing of $\approx 0.33 \mathrm{~nm}$ is an indicative of three ordered water layers adjacent to the silica and gibbsite nanoparticle basal plane. The analysis of the structural hydration force shows that the interfacial hydration layers are firmer (more organized) and thicker on gibbsite than on silica. Furthermore, $10 \mathrm{~nm}$ lateral resolution hydration maps reveal that gibbsite basal plane topographic imperfection and variation $\mathrm{pH}$ induce perturbations of the structural hydration forces. On amorphous silica, the oscillatory hydration forces are present only on $16 \%$ of the sample locations and are rather independent of the fluid composition.

\subsection{Introduction}

Any interaction between water and solid surfaces can substantially affect the properties of both phases. The structure and dynamics of the water molecules near the surface change. The reactivity and functionality of the substrate in contact with the water change as well [1] These interactions are thus of significant interest in many geochemical, technological and biological systems ${ }^{[2]}$. In geochemistry and environmental science, water-clay mineral interactions are important factors controlling processes such as surface ion adsorption and ion exchange as well as the transport and distribution of contaminants (radionuclides, heavy metals, or pesticides) in groundwater systems, soil moisture and the sequestration of atmospheric $\mathrm{CO}_{2}{ }^{[3,4]}$. The clay surface hydration structure also affects the adsorption/desorption of organic molecules from crude oils, changing the wetting characteristics and therefore the enhanced oil recovery process $[5,6]$. Water-clay interactions are largely controlled by the particle structure, chemical composition and surface charge distribution, which vary widely among minerals and individual clay particles $[3,7-10]$. The overall arrangement of near surface atomic species and the variation due to structural substitutions, (de)protonation or defects, result in neutral, negatively or positively charged surface sites. These sites can orient the $\mathrm{H}_{2} \mathrm{O}$ dipoles, influencing the hydrogen bonds to or from the surface, thus leading to a different density and $\mathrm{H}$-bond networking of the water, perpendicular and parallel to the surface. Absorbents, such as inorganic/organic ions and solution $\mathrm{pH}$ also affect the interfacial interactions and the hydration struc- 
ture. Yet, the mineral nanoparticles hydration structure is not well understood and is difficult to study experimentally. The intrinsic complexity of the minerals, the small particle sizes (a few nanometers) and the simultaneously exposed multiple surfaces, possibly with non-stoichiometric and heterogeneous compositions, hamper the systematic and accurate study of the water structure around clay nanoparticles using various techniques. Probing the interfacial water structure around surfaces using sum frequency generation [11,12] and polarimetric angle-resolved non-resonant second harmonic scattering [13], neutron reflectivity and X-ray spectroscopy and diffraction [14-17], requires preparing the surfaces in a suitable form with samples in a crystalline state, sufficiently large surface areas (typically larger than $0.1 \mathrm{~mm}$ ), and minimal heterogeneity and surface roughness. Hence, many investigations are performed on macroscopic homogeneous surfaces like mica, calcite or silica. Probing the structure of the water around clay nanoparticles is difficult not only experimentally, but also computationally. From a molecular dynamics (MD) simulation perspective, designing a reasonable representation of actual clay particles found in nature goes beyond the existing computational capability. For MD modeling is also challenging to include/account for variables, such as the $\mathrm{pH}$ and the electrostatic potential, acid-base properties of clay surfaces edge and basal planes [18-22]. In last 15 years, Atomic Force Microscopy (AFM) and spectroscopy have emerged as a powerful tool for topography imaging and characterization (surface charge, ions adsorption, adhesion, etc.) of the interface between solid surfaces / nanoparticles and aqueous solutions $[7-9,23,24]$. It has also been used to measure hydration forces of diverse surfaces including mica, calcite, graphite, complex DNA molecules and lipid bilayers with ${ }_{3} \mathrm{D}$ atomic resolution at room temperature $[25-31]$. Previous studies have already shown that the hydration force profiles or maps obtained by AFM gave a good qualitative description of intrinsic surface hydration structure and agree with the density distributions of the water molecules determined by the X-ray reflectivity or predicted by the MD simulations [32-34]. Herein, using Atomic Force Microscopy we map the hydration and the Derjaguin-Landau-Verwey-Overbeek (DLVO) forces with a lateral resolution of approximately $10 \mathrm{~nm}$ on an individual mineral nanoparticle of gibbsite $(\mathrm{Al}(\mathrm{OH}))$ adsorbed onto an oxidized silicon $\left(\mathrm{SiO}_{2}\right)$ wafer, in ambient electrolytes of variable $\mathrm{pH}$ and $\mathrm{NaCl}$ concentration. The force versus distance curves show oscillatory force profiles with a peak spacing of $\approx 0.33 \mathrm{~nm}$, indicating the existence of up to three ordered water layers adjacent to the gibbsite basal plane nanoparticles and silica substrate. Hydration maps, along with surface charge and topography maps, show 
that the strength and presence of structural hydration forces on a gibbsite basal plane strongly depend on topographic heterogeneity and $\mathrm{pH}$ of the solution (strongest at $\mathrm{pH}$ 6). The oscillatory hydration forces on an amorphous silica surface are less frequent compared to a crystalline gibbsite surface and are rather independent of fluid composition.

\subsection{Methods}

\section{Substrate and probe preparation}

The sample and cantilever is cleaned following the procedure described in chapter $4 \cdot 2$.

We use silicon probes (MikroMash $\mathrm{NSC}_{3} 6 / \mathrm{Cr}-\mathrm{Au} \mathrm{BS}$ ) covered by a 1-2 $\mathrm{nm}$ thick native oxide layer and a golden backside coating on the cantilever. Before use, the cantilevers are cleaned using the procedure described in chapter $3 \cdot 2 \cdot 1$.

\section{Amplitude modulation force spectroscopy}

Dynamic force spectroscopy measurements were performed with a commercial Asylum Research Cypher ES equipped with photo-thermal excitation [40,41] following the procedure described in chapter 4.2 . In imaging mode the topography of the sample is taken. From this large image (figure 5.7) a suitable particle for the force spectroscopy is chosen (dotted area in figure 5.7).

\section{Force inversion}

The conversion of amplitude and phase into tip-sample interaction forces is described in detail in chapter 4.2. Here, we only give the force inversion equation:

$$
k_{\text {int }}=k_{c}\left[1-\left(\frac{\omega}{\omega_{0}}\right)^{2}\right] \frac{A_{\infty} \cos \left(\varphi-\varphi_{\infty}\right)-A}{A}+\gamma_{c} \omega \frac{A_{\infty} \sin \left(\varphi-\varphi_{\infty}\right)}{A}
$$


where, $k_{\text {int }}$ is the interaction stiffness, $\omega=2 \pi f$ is the applied angular frequency, $\varphi$ the phase, A the amplitude and $\gamma_{c}$ the damping. The cantilever parameters (spring constant $k_{c}$, quality factor $Q$ resonance frequency $\omega_{0}$, effective mass $m^{*}=k_{c} / \omega_{0}^{2}$ and damping coefficient $\left.\gamma_{c}=k_{c} /\left(\omega_{\circ} Q\right)\right)$ are determined during the experiment for each fluid composition at $D=20 \mathrm{~nm}$, where the tip-sample interaction is negligible (see figure 5.8). Integrating $F(z)=\int_{z}^{\infty} k_{\text {int }}\left(z^{\prime}\right) d z^{\prime}$, leads to the tip-sample interaction force. During the extraction of the tip from the AFM probe holder and cleaning the tip was damaged and it could not be imaged by Scanning Electron Microscopy (SEM). In order to retrieve the tip radius, another approach had to be taken. From previous experiments with identical experimental settings, tip type and conditions we know very accurately the silica charge at $\mathrm{pH} 5.8$ and $10 \mathrm{mM} \mathrm{NaCl}$. Therefore, we calculated the FAM tip radius by fitting (tip radius as fitting parameter) the model electrostatic interaction to the experimental silica-silica force curves for the $\mathrm{pH} 6$ and $10 \mathrm{mM} \mathrm{NaCl}$. This results in a tip radius of $\approx 9 \mathrm{~nm}$. The other tip parameters are $k_{c} \approx$ $1 \mathrm{~N} \mathrm{~m}^{-1}, \omega_{\infty} \approx 30 \mathrm{kHz}$ and $Q \approx 3.5$.

\section{Surface charge calculation}

We use DLVO-theory including van der Waals and electric double layer forces and a charge regulation model for tip and substrate surface chemistry, to extract the diffuse layer charge from the measured force-distance curves $[24,42-45]$. We follow the procedure as described in chapter 4.2. Here, we only give the final expression for the charge density $\sigma_{\text {eff }}$ as a function of the surface potential $\psi_{s}$ for silica and gibbsite as obtained from the charge regulation model (table 5.1). Since we have a spatial distribution of the force-distance curves, the charge fitting results in a $2 \mathrm{D}$ map of the diffuse layer charge.

As discussed in chapter 4.2, the obtained $\mathrm{pK}$ values depend on the assumed set of surface reactions (surface complexation equations) and are not necessarily unique and always reliable. The resulting effective surface charge turns out to be insensitive to the assumed set of surface reactions, because the double layer contribution of the measured interaction force $F_{e d l}(h)$ depends only indirectly via the charge density $\sigma$ substrate on the fit parameters $K_{j}$. Therefore, we will only discuss the effective surface charge $\left(\sigma_{e f f}=\sigma_{\circ}+\sigma_{S}\right)$ or the diffuse layer charge $\left(\sigma_{D}\right)$ and not the $\mathrm{pKi}$ values. The diffuse layer charge is related to the surface charge with $\sigma_{\circ}+\sigma_{S}=-\sigma_{D}$. 


\begin{tabular}{cc}
\hline Reactions & Charge \\
\hline$S H \rightleftharpoons S^{-}+H^{+} ; K_{H}$ & $\sigma_{\text {eff }}=\frac{-q_{e} \Gamma}{1+A \exp \left(-\frac{q_{e} \varphi_{s}}{k_{B} T}\right)}$ \\
$S C \rightleftharpoons S^{-}+C^{+} ; K_{C}$ & $A=10^{p K_{H}\left[H^{+}\right]_{\infty}+10^{p K_{C}\left[C^{+}\right]_{\infty}}}$ \\
\hline$=\left\{S^{-}\right\}+\{S H\}+\{S C\}$ &
\end{tabular}

Table 5.1: Surface chemistry equations and final charge regulation condition for the silica surface as used by the $2 p K$ surface complexation model $\left(p K_{X}=-\log _{10} K_{X}\right)$. The charge mentioned refers to the effective surface charge.

\subsubsection{Hydration Forces}

The force gradient data between the AFM tip and the silica-gibbsite sample were fitted using two methods. First, as described above and in our previous publications $[7,8,24]$, the recorded force-distance curves were analyzed in the framework of the DLVO theory in combination with a charge regulation boundary conditions. This allowed us to quantify the $2 \mathrm{D}$ spatial distribution of the diffuse layer charge of the silica-gibbsite sample and its dependence on the fluid composition. Second, the total force gradient was fitted using a function consisting of a superposition of a DLVO and a hydration force (equation 5.2). This fitting step enabled us to quantify the strength and decay length of the EDL force (surface charge) and hydration force as function of fluid composition and lateral position on the sample.

$$
F_{T O T}(D)=F_{D L V O}+F_{H Y D}=F_{E D L}+F_{v d W}+F_{H Y D}
$$

The Van der Waals interaction, $F_{v d W}$, between an AFM probe with radius $R$ and a flat surface is described as $F_{v d W}=-A_{H} R /\left(6 \pi D^{2}\right)$. Here, $A_{H}$ is the Hamaker constant and $D$ is the tip to surface distance.

The electrical double-layer interaction was modeled by a simple exponential:

$$
F_{E D L}=A_{E D L} e^{\frac{-D}{\lambda_{E D L}}}
$$

where $A_{E D L}$ is the amplitude of the electrical double-layer force, and $\lambda_{E D L}$ is the range of this interaction. The hydration force $\left(F_{H Y D}\right)$ is described as linear combination of 
a monotonically decaying exponential function (monotonic hydration force) and a decaying oscillatory contribution (structural hydration force) arising from the presence of ordered water layers [49-[51] .

$$
F_{H Y D}=F_{H Y D \_M O N}+F_{H Y D \_O S C}=A_{o s c} \cos (2 \pi \sigma D-\varphi) e^{\frac{-D}{\lambda_{o s c}}}+A_{m} e^{\frac{-D}{\lambda_{m}}}
$$

$$
\frac{d F_{H Y D}}{d D}=\sqrt{(2 \pi \sigma)^{2}+\lambda_{o s c}^{-2}} A_{o s c} \cos \left(2 \pi \sigma D-\varphi-\arctan \left(2 \pi \sigma \lambda_{o s c}\right)\right) e^{\frac{-D}{\lambda_{o s c}}}+\frac{A_{m}}{\lambda_{m}} e^{\frac{-D}{\lambda_{m}}}
$$

where $A_{o s c}$ and $A_{m}$ are the magnitude of the structural and the monotonic hydration forces, $\varphi$ is the phase shift, $\sigma$ is the structural hydration layer spacing, and $\lambda_{\text {osc }}$ and $\lambda_{m}$ are the decay lengths of the structural and monotonic hydration force. The structural hydration force is typically described by an exponentially decaying cos-function and originates ascribed to the force required to displace layers of structured water molecules in between the AFM tip and surface. The oscillation region extends over a few $\left(\approx_{3}\right)$ molecular diameters, while monotonic hydration force extends over a larger region of space (up to $1.5-2 \mathrm{~nm}$ ). Yet, the origin of a monotonically decaying hydration force (also called primary hydration force) is less clear and different mechanisms have been proposed $[47,52]$.

\section{$5 \cdot 3$ Results}

Macroscopic characterization of a nanoparticle Atomic force microscopy topography analysis shows that the gibbsite nanoparticles have the typical plate-like pseudo hexagonal morphology with lateral dimensions ranging from 100 to $500 \mathrm{~nm}$ and heights from 5 to $20 \mathrm{~nm}$ (Figure $5.11 \mathrm{a}, \mathrm{b}$ and 5.7 ). The AFM images also reveal that the majority of nanoparticles (001) basal plane appears smooth across 20 $-100 \mathrm{~nm}$ regions (terraces in figure $5.1 \mathrm{a}$ and figure 5.7 ), separated by irregular areas containing steps and other defects [23] (figure 5.1 a and figure 5.7). Overall, the number of surface imperfections is enhanced at the boundaries of the particles.

3D force field measurements As described in great details in our earlier publications [7-9] ${ }_{3} \mathrm{D}$ AM-AFM force mapping is used to probe the spatial distribution 
of the forces on the silica-gibbsite sample during exposure to a variety of electrolyte solutions. A $2 \mathrm{D}$ projection of the force gradient (extracted from ${ }_{3} \mathrm{D}$ map at $2 \mathrm{~nm}$ separation) shows that at $\mathrm{pH} 6$ and $10 \mathrm{mM} \mathrm{NaCl}$ the interactions are repulsive on silica and mainly attractive on gibbsite (figure 5.1 a). All force-distance (FD) curves are probed with a negatively charged silica tip, which implies that the gibbsite surface is positively charged. The measurements also reveal a significant heterogeneity in the force distribution on the gibbsite basal plane, as compared to silica (figure 5.1 a). The forces are less attractive near topographic defects and rim of the particle, consistent with our previous observations $[7,8,8,24]$. All ${ }_{3} \mathrm{D}$ maps are measured with $10 \mathrm{~nm}$ lateral resolution using a silica tip with radius of $9 \pm 2 \mathrm{~nm}$. In the line representation of force-distance curves (Figure $5.1 \mathrm{c}$ and $\mathrm{d}$ ), three regions can be distinguished: (1) $7>\mathrm{D}<1.5 \mathrm{~nm}$; (2) $1.5>\mathrm{D}>0.1 \mathrm{~nm}$; and (3) $\mathrm{D}<0.1 \mathrm{~nm}$. At tip sample separation $7 \mathrm{~nm}>\mathrm{D}<15 \mathrm{~nm}$ a monotonic attraction on gibbsite and repulsion on silica is observed. This force is caused by the electrostatic double layer (EDL). It decays exponentially with a decay of $2.6 \mathrm{~nm}$ (figure 5.8), which closely agrees with the Debye screening length ( $3 \mathrm{~nm}$ at $10 \mathrm{mM} \mathrm{NaCl}$ ). At smaller separations $1.5 \mathrm{~nm}>\mathrm{D}>0.1 \mathrm{~nm}$ an oscillatory (non-DLVO) force, with up to three maxima $\approx 0.3 \mathrm{~nm}$ apart, is detected. Due to the similarity of the peak distance to the size of a water molecule, we assume that oscillations in the force profiles arise as water molecules are sequentially displaced from discrete ordered interfacial layers. The oscillatory force profile is present until a separation of $\approx 0.1 \mathrm{~nm}$ and then a strong repulsive force in the constant compliance region is detected (region 3 ). Commonly, the oscillatory force or structural hydration force was mostly measured on rigid atomically smooth surfaces like mica and calcite $[26,34,53]$. Here, we were able to detect for the first time the oscillatory hydration force on gibbsite crystalline basal plane and on amorphous silica as well. On the gibbsite, $62 \%$ of the FD curves show oscillations and are primarily located on topographically smooth terraces as indicated by circles in figure 5.1 a. On the silica, only $12 \%$ that are randomly distributed across the sample (crosses on figure 5.1 a). In the rest of the sample locations ( $88 \%$ silica and $38 \%$ gibbsite) in region 2 , the FD curves display a monotonically decaying non-DLVO force (figure 5.1 d). In these locations water molecules are also structured differently from bulk water, yet are not ordered into discrete layers to present multiple energy barriers to approaching nanoscale tip.

Surface force: Effect of $\mathbf{p H}$ and ion concentration The same distinct regions, 

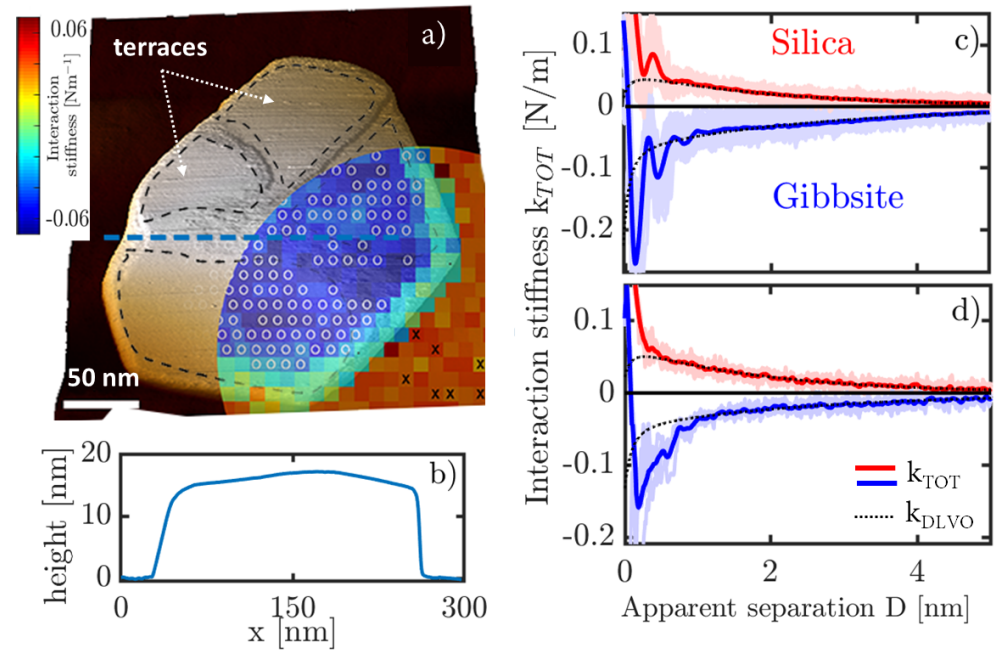

Figure 5.1: a) AFM topography of a gibbsite particle on silica superimposed with a partial map of the interaction stiffness measured at $2 \mathrm{~nm}$ from the surface in a $10 \mathrm{mM} \mathrm{NaCl} \mathrm{pH} 6$ solution. The circles and crosses indicate the locations offorce distance approaches with an oscillatory behavior. The total interaction stiffness vs tip sample separation from locations that show (c) and do not show oscillatory behavior (d). The shaded regions are the 10 individual interaction stiffnesses. The thick red and blue colored lines are their respective averages. The black dotted lines are the DLVO interaction. 
with a long-range EDL and pronounced oscillatory interaction stiffness at separation below $1.5 \mathrm{~nm}$ are observed in measurements at $\mathrm{pH}_{4}, 6,9$ with 10 and $100 \mathrm{mM} \mathrm{NaCl}$ solutions (figure 5.2). Solid colored lines in figure 5.2 represent the average force gradient of 10 individual FD approaches that display an oscillatory behavior extracted from ${ }_{3} \mathrm{D}$ force maps. All six $3 \mathrm{D}$ force maps are recorded on the same gibbsite particle with the same probe and great care was taken to guarantee that tip size did not change during the measurements and when solutions were exchanged (see Methods and Materials for details). As shown in Figure 5.9, force gradient at beginning and after completing all six ${ }_{3} \mathrm{D}$ force maps (7000 FD approaches) overlap, indicating that the tip size did not change during the whole experiment. Data presented in figure 5.1 and 5.2 indicate that with $\mathrm{SiO}_{2}$ tip with radius of $9 \pm 2 \mathrm{~nm}$ is suitable for simultaneously mapping the oscillatory hydration and DLVO forces at level of single nanoparticle under various fluid conditions. The tip size and geometry of the apex and hydration play a critical role for simultaneous detection of DLVO and hydration forces, in particular oscillatory ones $[54-57]$. As shown in our earlier publications and others, tips of $25 \mathrm{~nm}$ have a very good sensitivity of DLVO force, while oscillations $[8,24]$ of hydration force are completely averaged out. Measurements with very sharp tips of $1-2 \mathrm{~nm}$ can reveal the structural hydration force in $3 \mathrm{D}$ with sub-nanometer lateral resolution. Yet, very sharp tips are typically not sensitive to long range electrostatic forces (EDL), and therefore correlations between the surface charge and hydration force cannot be made ${ }^{[26]}$. Very few experiments, where the EDL and structural hydration forces were probed simultaneously, were done using SFA, that lacks lateral resolution and suffers from confinement effects over a laterally extended contact area $[50,58-61]$.

Fitting of force distance curves The qualitative trends were captured in quantitative numbers by fitting the total interaction stiffness $\left(k_{T O T}\right)$ using a function consisting of a superposition of a DLVO interaction $\left(k_{D L V O}\right)$ and a hydration interaction $\left(k_{H Y D}\right)$ (equation 5.2 in Methods and Materials). Using only DLVO theory in combination with a charge regulation boundary condition allowed us to quantify the effective surface charge (figure 5.4) [7, 24]. However, as expected and shown previously, the continuum theories of Van der Waals force and double-layer force $\left(k_{E D L}\right)$ cannot describe the interactions at distances smaller than 1.5-2 nm (black dotted lines in figure 5.2). Good fits, down to separations of $\approx 0.15 \mathrm{~nm}$ are obtained only if along with DLVO $\left(k_{D L V O}\right)$ a monotonic $\left(k_{H Y D}\right.$ MON $)$ and oscillatory structural hydration 


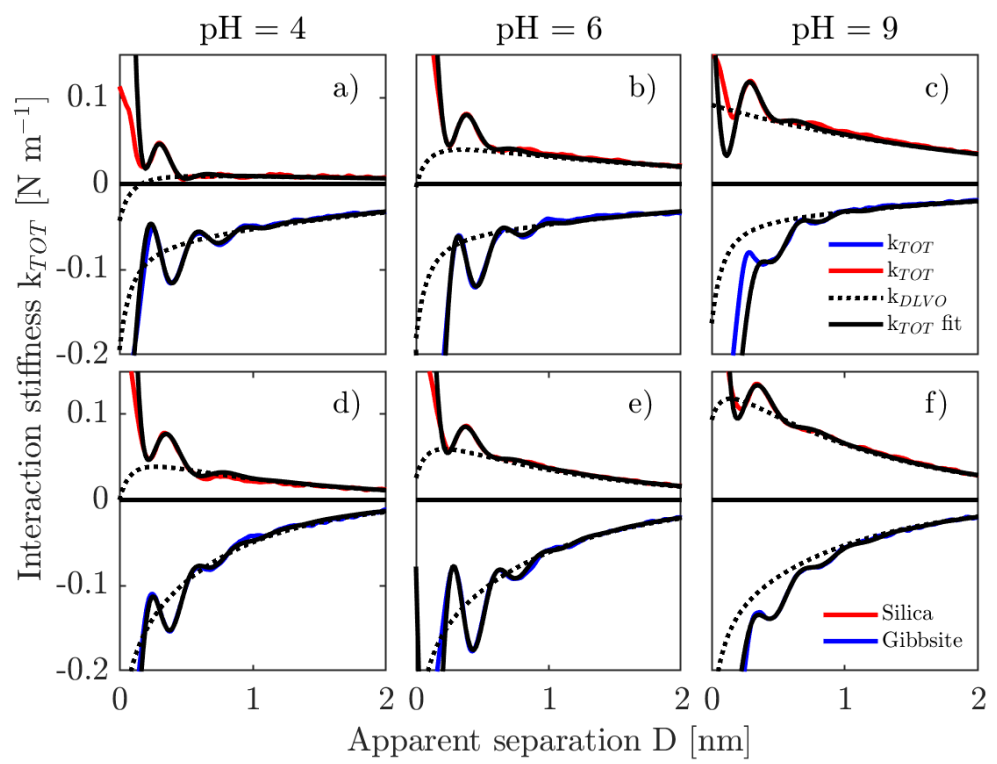

Figure 5.2: The average interaction stiffness $\left(k_{T O T}\right)$ from the 10 individual approaches for $10 \mathrm{mM}$ (top row) and $100 \mathrm{mM}$ (bottom row) $\mathrm{NaCl}$ in various $\mathrm{pH}$ solutions. The black solid lines are the best fits using a function consisting of a superposition of a DLVO interaction $\left(k_{D L V O}\right)$ and hydration interactions $\left(k_{H Y D}\right)$ (equation 5.2). The dotted black line is the DLVO interaction $\left(k_{D L V O}\right)$. 
force $\left(k_{\text {HYD_OSC }}\right)$ is taken into account (black solid lines in figure 5.2). The structural hydration force is typically ascribed to the force required to displace layers of structured water molecules and is described by an exponentially decaying cos-function. The monotonically decaying hydration force (also called primary hydration force) observed in many biological systems is described by a monotonically decaying exponential function, though its origin is still under debate [47,552]. The parameters (strength, decay length of the EDL and hydration forces, oscillatory wavelength) extracted from data fitting at different fluid compositions are listed in Table 5.2. Spatial $2 \mathrm{D}$ distributions of the fitting parameters across the silica-gibbsite sample are plotted in figure 5.4. Individual components $\left(k_{H Y D \_M O N}, k_{H Y D \_O S C}, k_{E D L}\right)$ of the total force gradient $\left(k_{T O T}\right)$ are shown in the figures 5.3, 5.10 and 5.11. The analysis of AFM data shows that the EDL force gradient $\left(k_{E D L}\right)$ between the $\mathrm{SiO}_{2}$ tip and the $\mathrm{SiO}_{2}$ substrate is repulsive and increases in strength $\left(A_{E D L}\right.$ in table 5.2) with rise in $\mathrm{pH}$ and $\mathrm{NaCl}$ concentration (figure 5.10). This is a result of a negative charge increase of the silica (figure 5.6), since the fraction of deprotonated $\mathrm{SiO}^{-}$groups rises with increasing $\mathrm{pH}$ and $\mathrm{NaCl}$ concentration. On gibbsite, $k_{E D L}$ is attractive (positive effective surface charge) and heterogeneous under all investigated conditions and decreases with increasing $\mathrm{pH}$, from 4 to $9\left(A_{E D L}\right.$ in Table 5.2 and figure 5.3). The decay length of the EDL interaction, $\lambda_{E D L}$ decreases from $2.4 \pm 0.4 \mathrm{~nm}$ to $1.07 \pm 0.17 \mathrm{~nm}$ with increasing salt concentration from 10 to $100 \mathrm{mM}$ (similar to the Debye length $\lambda_{D}=3 \mathrm{~nm}$ and $0.96 \mathrm{~nm}$ ). Also as expected, the EDL thickness $\lambda_{E D L}$ is independent of $\mathrm{pH}$, surface properties and the sign of surface charge (See figure 5.4 and Table 5.2). Small deviation at low concentration from theoretically calculated Debye length could be either due to the lower force sensitivity at large separations, due to non-perfect fluid exchange in the measuring cell, or dissolved airborne $\mathrm{CO}_{2}$.

Subtraction of the DLVO from the total force gradient shows that the hydration force $\left(k_{H Y D}\right)$ consists of a superposition of a monotonically decay $\left(k_{H Y D}\right.$ MON $)$ and an oscillatory part $\left(k_{\text {HYD_OSC }}\right)$ (figure $5.3 \mathrm{c}$ and d). Both contributions have a rather heterogeneous spatial distribution across silica-gibbsite sample (figure 5.4). The monotonic hydration force in all fluid compositions is attractive on gibbsite and repulsive on silica (figure 5.11). The decay lengths of the oscillatory and the monotonic contributions for silica are $\lambda_{\text {mon }}=0.075 \pm 0.03 \mathrm{~nm}$ and $\lambda_{\text {osc }}=0.15 \pm 0.02 \mathrm{~nm}$ (Table 5.2). For gibbsite these values are larger $\lambda_{\text {mon }}=0.25 \pm 0.06 \mathrm{~nm}$ and $\lambda_{\text {osc }}=0.23 \pm 0.03 \mathrm{~nm}$ indicating a thicker interfacial hydration layer. Compared to the EDL force, the de- 

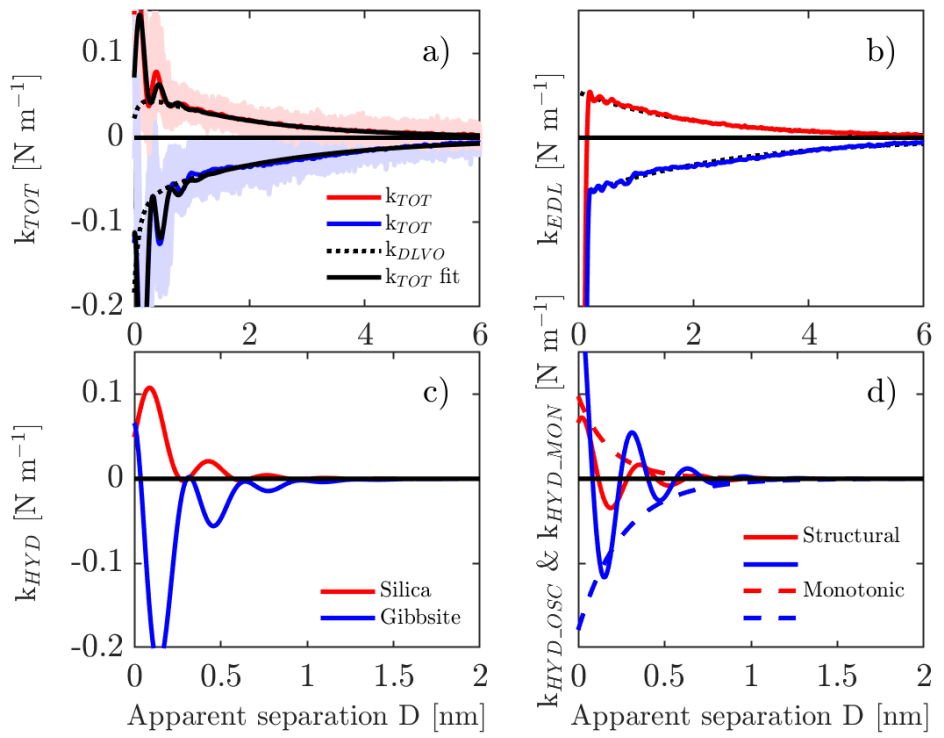

Figure 5.3: a) The shaded regions are the total interaction stiffnesses from the 10 individual approaches on silica (red) and gibbsite (blue). Their respective averages are shown as a colored line. The black lines are the fit, which includes the DLVO force and the structural and monotonic part of the hydration force. The dotted lines are the DLVO interaction. b) The EDL interaction after subtracting the hydration and van der Waals force gradient. c) Total hydration force gradient on silica (red) and gibbsite (blue). d) The structural (solid) and monotonic (dashed lines) hydration forces shown separately. 
cay length of hydration forces does not vary of salt concentration and $\mathrm{pH}$ but depends on surface properties. This indicates that the behavior of the hydration forces is not caused by continuum electrostatics, otherwise its range would vary with the salt concentration. This agrees with recent measurements and analysis reported by van Lin et al. [49], where the decay length of hydration forces (oscillatory $\approx 0.2 \pm 0.08$ and monotonic $0.4 \pm 0.1 \mathrm{~nm}$ ) between a sharp silica tip and mica surface are independent of fluid composition ( $\mathrm{pH}$ and monovalent salts concentration from 0.001 to $4 \mathrm{M}$ ). The absolute magnitude of the monotonic hydration force is more sensitive to the fitting analysis and strongly depends on the subtle subtraction of the Van der Waals force [62,63]. Therefore, the following discussion is primarily focused on the structural component of the hydration force [62,63]. On gibbsite, we can distinguish three hydration layers, whereas on silica only 2 are visible with a smaller amplitude ( $A_{H Y D}$ OSC in Table 5.2) (figure 5.6). The distance measured between the adjacent hydration layers for gibbsite is $0.34 \pm 0.01 \mathrm{~nm}$ and rather independent of solution $\mathrm{pH}$ and $\mathrm{NaCl}$ concentration. For silica these distances are substantially larger $0.42 \pm 0.03 \mathrm{~nm}$ (Table 5.2). The amplitude of the oscillatory hydration forces and their periodicity is typically associated with organization of water within the hydration layers. A higher oscillation amplitude and a closer periodicity to the diameter of the water molecule means a higher strength/order of the hydrogen bond (HB) network between water molecules within ordered hydration layers. Thus, the thickness and organization of water within the gibbsite hydration layers is higher than amorphous silica surface.

There are at least two possible reasons for this: first, the amorphous silica surface is not as smooth as the crystalline surface of gibbsite. Therefore, the $\approx_{2}$ Angstroms of surface roughness may overwhelm the layering of water molecules and oscillatory hydration force. Second, different distribution and distance between water hydrogenbonding sites could be responsible for different hydration structures observed on the two surfaces $[54,64,65]$. The proton reactive site density on gibbsite basal planes is 8 to $13 \mathrm{~nm}^{-2}$, which is twice as much for amorphous silica, which is 5 to $8 \mathrm{~nm}^{-2}$. These sites are relatively far from each other $(\approx 0.5 \mathrm{~nm})$ even on crystalline silica, as shown by Molecular dynamics (MD) simulations. Therefore, the water molecules adsorbed on the first hydration layer are not likely to form HBs among themselves. On the other hand, the sites are at $\approx 0.3 \mathrm{~nm}$ from each other on alumina [54] and $0.27 \mathrm{~nm}$ on hematite [65]. This facilitates the formation of HBs between the water 


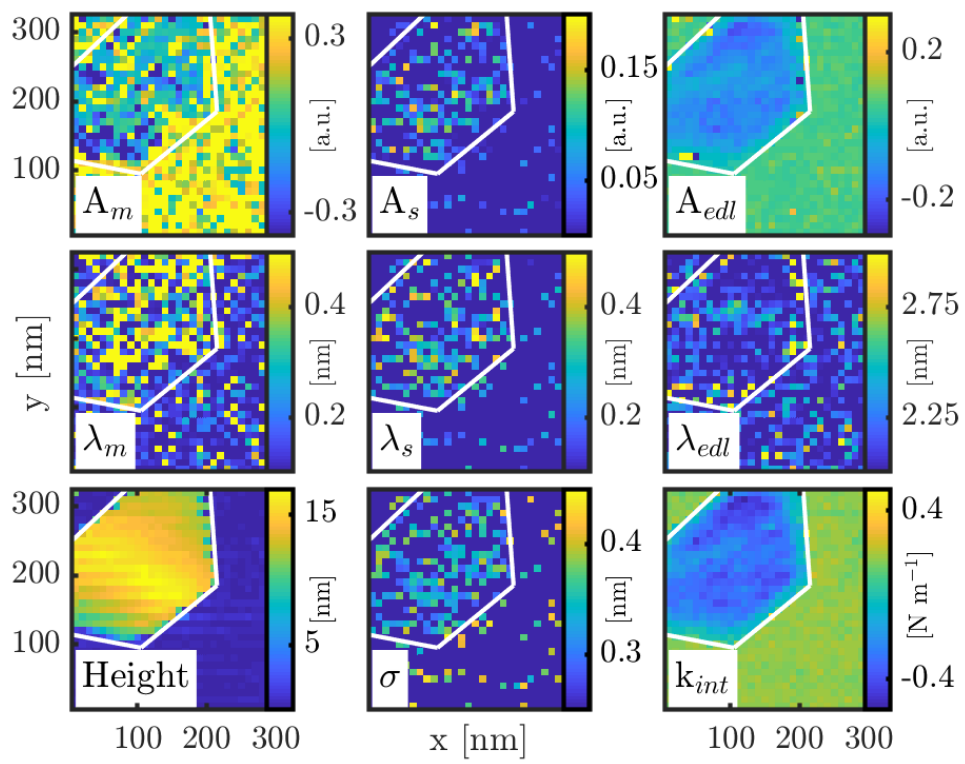

Figure 5.4: The fitting parameters for gibbsite on silica in a $10 \mathrm{mM} \mathrm{NaCl} \mathrm{pH} 6$ solution using equation 5.2. The top row shows the amplitudes of the monotonic hydration force, the structural hydration force and the electric double layer force. The middle row shows the decay lengths of the monotonic hydration force, the structural hydration force and the electric double layer force. The bottom row shows the height, oscillation period and measured total interaction stiffness at $2 \mathrm{~nm}$ from the surface. 
molecules within the first hydration layer along with the solid substrate and gives rise to a highly organized first hydration layer. Consecutively, water-water HBs determine the order in consecutive hydration layers, and also how far the perturbation of the water structure persists.

\begin{tabular}{l|ccc|ccc|cc}
\hline \multicolumn{7}{c}{ Silica } \\
\hline $\mathrm{pH} / \mathrm{c}$ & $\mathrm{A}_{\text {edl }}$ & $\mathrm{A}_{\text {osc }}$ & $\mathrm{A}_{m}$ & $\lambda_{\text {edl }}$ & $\lambda_{\text {osc }}$ & $\lambda_{m}$ & $\sigma$ & $\varphi$ \\
\hline $4 / 10$ & 0.01 & 0.011 & 0.06 & 3.03 & 0.07 & 0.15 & 0.36 & 4.73 \\
$6 / 10$ & 0.05 & 0.010 & 0.03 & 2.05 & 0.13 & 0.15 & 0.37 & 4.82 \\
$9 / 10$ & 0.09 & 0.010 & 0.02 & 2.04 & 0.20 & 0.15 & 0.35 & 4.91 \\
$4 / 100$ & 0.06 & 0.030 & 0.08 & 1.25 & 0.08 & 0.16 & 0.50 & 4.51 \\
$6 / 100$ & 0.08 & 0.011 & 0.02 & 1.19 & 0.09 & 0.15 & 0.40 & 4.94 \\
$9 / 100$ & 0.15 & 0.011 & 0.16 & 1.20 & 0.03 & 0.18 & 0.45 & 4.29 \\
\hline & & & $\mathrm{Gibbsite}$ & & & & \\
\hline $4 / 10$ & -0.08 & 0.015 & -0.04 & 2.24 & 0.20 & 0.22 & 0.35 & 4.68 \\
$6 / 10$ & -0.07 & 0.025 & -0.09 & 2.64 & 0.17 & 0.19 & 0.34 & 4.92 \\
$9 / 10$ & -0.05 & 0.010 & -0.10 & 2.18 & 0.18 & 0.18 & 0.34 & 4.79 \\
$4 / 100$ & -0.17 & 0.017 & -0.06 & 0.80 & 0.20 & 0.18 & 0.32 & 5.20 \\
$6 / 100$ & -0.17 & 0.030 & -0.06 & 0.96 & 0.20 & 0.19 & 0.33 & 4.98 \\
$9 / 100$ & -0.14 & 0.010 & -0.07 & 1.00 & 0.20 & 0.17 & 0.32 & 5.18 \\
\hline
\end{tabular}

Table 5.2: Fit parameters from figure 5.4, extracted using equation 5.5 while fitting the experimental interaction stiffness data.

Simultaneous detection of EDL and hydration forces also reveal that the diffuse layer charge and oscillatory hydration force are not correlated and respond very differently to $\mathrm{pH}$ and salt concentration variation. The gibbsite effective surface charge monotonically decreases from +0.038 to $+0.021 \mathrm{e} / \mathrm{nm}^{2}$ with $\mathrm{pH}$ increase from 4 to 9 (Figure 5.5). On the other hand, the strength ( $\left.A_{\text {HYD_OSC }}\right)$ of the oscillatory hydration force (the degree of order within the hydration layers) on the gibbsite has a non-monotonic $\mathrm{pH}$ dependence and is highest at $\mathrm{pH} 6$ (Figure 5.6). On silica, the strength of the oscillatory hydration force is rather independent of fluid composition, while its effective surface charge increases twice (from -0.073 to $-0.2 \mathrm{e} / \mathrm{nm}^{2}$ ) when $\mathrm{pH}$ changes from 4 to 9 and also depends on the salt concentration. As indi- 


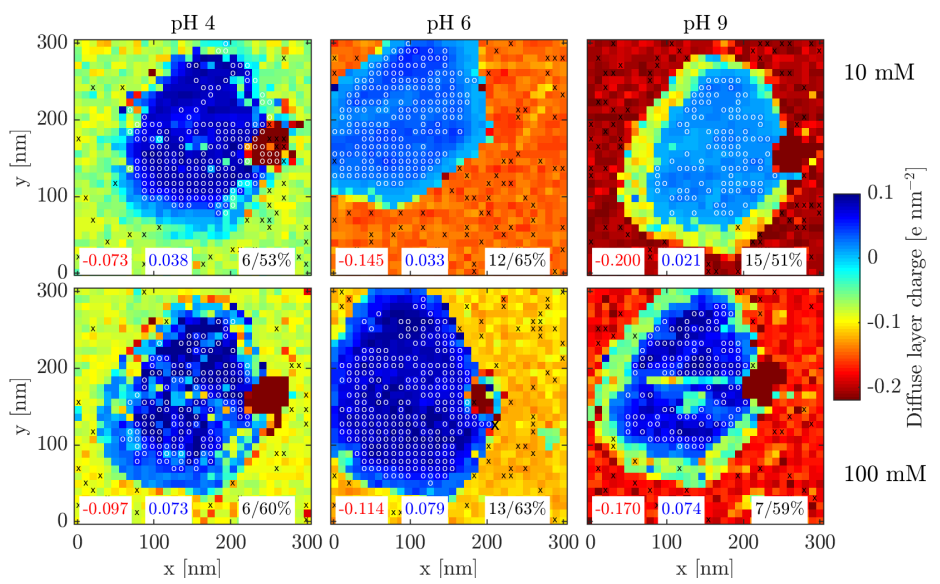

Figure 5.5: Diffuse layer charge maps $\left(-\sigma_{D}\right)$ of the same gibbsite particle on silica in various electrolyte solutions. The bottom left corner indicates the average effective surface charge for silica (red) and gibbsite (blue). The bottom right shows the percentage of approaches that show oscillatory behavior on silica (left) and gibbsite (right). The white circles and black crosses indicate the locations of FD approaches with oscillatory behavior on gibbsite and silica, respectively. 


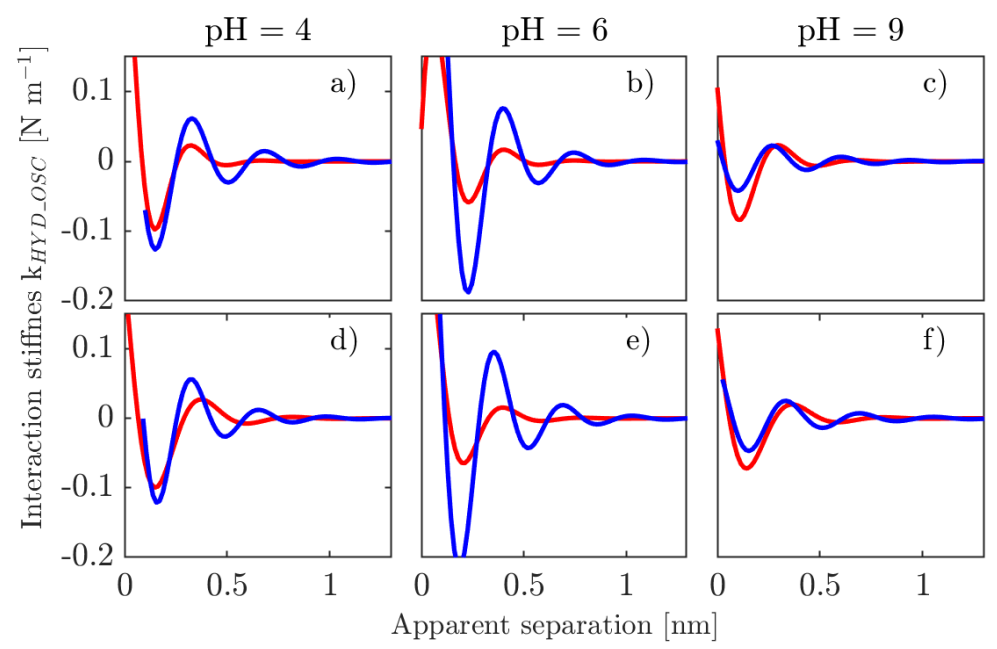

Figure 5.6: The structural hydration force gradient as function of apparent separation for different fluid compositions for silica (red) and gibbsite (blue). Top row is $10 \mathrm{mM} \mathrm{NaCl}$ and the bottom row is $100 \mathrm{mM} \mathrm{NaCl}$.

cated with the circles and crosses in figure 5.6, the surface charge variation does not affect the number and spatial distribution of forces that exhibit an oscillatory profile. On gibbsite, $\approx 58 \%$ of forces display oscillatory character and are overall located on regions with a flat topography. This is rather independent of electrolyte composition. The areas with topographic defects smear out the oscillatory force, leaving only a monotonic component. On silica, despite the homogeneous surface charge distribution and surface roughness, the structural hydration forces are present only on $\approx 10 \%$ random locations.

\subsection{Discussion}

The above experimental observations and trends can be rationalized as follows (figures 5.1 5.6). The absolute value of the gibbsite surface ionization is very small. A surface charge value of $+0.04 \mathrm{e} / \mathrm{nm}^{2}$ corresponds to only 1 positive charge in about 40 unit cells. In other words, the average distance between charged sites on the gibb- 
site surface is in the order of a few nanometers. Such a large separation between these sites and the water-water correlation length of $\lambda=3$ to $5 \AA$ (distance over which structuring of water propagates into the solution) suggest that the gibbsite surface charge is not the dominant factor responsible for the organization of water near surfaces and therefore the structural hydration force. It is more plausible that the hydration structure originates from hydrogen bonding of water molecules with - $\mathrm{OH}$ groups above the aluminum $(\mathrm{Al})$ atom. As predicted by molecular dynamics simulations [66,67], three - OH groups (proton donors) point into the solution and form hydrogen bonds with water oxygens. The other three $-\mathrm{OH}$ groups (proton acceptors) align parallel to the surface and can form hydrogen bonds with water hydrogens. This leads to a highly organized first water layer and with a net dipole moment where the negative end of the water molecule points toward the surface and follows the gibbsite hexagonal lattice arrangements. These ordered hydration layers can extend up to $1 \mathrm{~nm}$ above the surface and give rise to oscillations in the measured force gradient (Figure 5.5). According to multi-site complexation (MUSIC) model developed by Bickmore et al. [68,69] one of six different types of doubly coordinated hydroxyl groups $\left(\equiv \mathrm{Al}_{2} \mathrm{OH}\right)$ in each unit cell should have $\mathrm{pK}$ values of 5.2 and could deprotonate/protonate at $\mathrm{pH}$ higher/lower than 6. Therefore, at $\mathrm{pH} 9$ these sites transform from proton donors to only proton acceptors (opposite to $\mathrm{pH} 4$ ) that will reorient the water molecule with respect to their neighbors and destabilize the hydrogen bonding of interfacial water layers. Such disorder in the hydration layers is captured by a reduction in the amplitude of the oscillatory structural hydration force. The presence of microscopic defects and their associated singly coordinated $-\mathrm{OH}$ groups (designated $\equiv \mathrm{AlOH}$ ), with a $\mathrm{pK} \approx 6$ within the probing area, can also lead to the same effect. For silica, the origin of the hydration forces is a controversial topic, despite extensive studies for more than a century [70]. In all previous studies only monotonic non-DLVO forces have been measured experimentally. Therefore, often, this non-DLVO force was assigned to elastic deformation of polysilicilic acid chains (or 'silica hairs') protruding from the silica surface $[2,71,72]$, rather than to the hydration structure. In our measurements, the oscillatory character with the periodicity close to a water molecule indicates that the force originates from structured hydration layers. Independence of hydration force on $\mathrm{pH}$ and $\mathrm{NaCl}$ concentration suggests that charged $\mathrm{SiO}^{-}$groups do not significantly contribute to the silica structural hydration effects. Otherwise, the increase of $\mathrm{pH}$, and thus the degree of silica surface charge would lead to an increase in the strength of hydration force (increase 
of orientational water polarization and stability of hydration layers) [73,74]. Therefore, consistent with recent MD simulations and experiments of Horn et al. [72,75] and Chapel [59], water hydrogen bonding to undissociated silanols $(\mathrm{SiOH})$ groups (water molecules are oriented hydrogen-down) is the main factor responsible for the hydration structure and force. This is not surprising, as the total degree of deprotonation is rather low for the conditions of our experiments. Given the typical density of 8 sites per $\mathrm{nm}^{2}$, the free deprotonated $\mathrm{SiO}^{-}$groups at $\mathrm{pH} 4$ is only $0.2 \%$ and will increase to $2 \%$ at $\mathrm{pH} 9$ and $100 \mathrm{mM} \mathrm{NaCl}$. Also, $30 \%$ of $\mathrm{SiOC}^{+}$complexes will not affect the hydration force much, since silanolate- $\mathrm{Na}^{+}$coordination reduces the silanolate-water (hydrogen) coordination number only slightly from 2.83 (o mM) to 2.67 (200 $\mathrm{mM}$ ionic strength) [76].

The locations (random 13\%) where forces display an oscillatory profile could be due to the patchy $(\mathrm{SiOH})$ rich regions or it is simply a local surface roughness effect. $\mathrm{MD}$ simulations show that amorphous silica has patchy and non-uniform distribution of silanol ( $\mathrm{SiOH}$ ) rich (hydrophilic sites) and siloxane ( $\mathrm{SiOSi}$ )-rich (hydrophobic sites-weakly accept hydrogen bonds $\left[{ }^{[7]}\right)$ domains. Therefore, the adsorbed water forms a hydrogen-bonding network with intermingled regions of ordered and disordered structures $[12,78-80]$. However, more detailed experiments are required to know whether the regions with forces that display oscillations are $(\mathrm{SiOH})$ rich or it is a roughness effect. The attractive monotonic hydration force on gibbsite can be rationalized based on different orientation of the water of interacting surfaces. Overall, on silica, water molecules are oriented with their hydrogen atoms toward the silica surface. On gibbsite it is the opposite and the negative oxygen of the water molecules points towards the surface. According to Marcelja and Radic [81,82] model, hydration forces between two interacting surfaces (silica tip and gibbsite) with an opposite water dipole orientation will be attractive, since hydrogen-bonding structure of the intervening water is strengthened. Opposite, the force between two surfaces that polarize water in the same direction (silica tip and silica substrate), is predicted to be monotonically repulsive, as the hydrogen-bonded network will become increasingly disrupted as surfaces move closer. In summary, we show for the first time that with the right tip size it is possible to probe the nanoparticle topography, along with hydration structure and surface charge (landscape) and their correlation simultaneously. Data analysis allows us to see how individual components of the force $\left(k_{H Y D}\right.$ MON, $\left.k_{H Y D \_O S C}, k_{E D L}\right)$ depend on fluid composition and allows us to establish the relation 
between the diffuse layer charge and the hydration force $[83,84]$, that until now was not addressed explicitly. This contributes to the understanding of silica and gibbsite interfacial properties that are paramount for their further applications. Extension of the present experimental approach and analysis may help to explain a number of macroscopic observations where the existence and the effect of hydration forces have been more often inferred than measured. For example, in colloidal science there are significant uncertainties about the relative importance of DVLO vs. hydration forces in the stability of particle dispersions, especially weakly charged particles and/or at high electrolyte concentrations, when the double layer is expected to collapse. The door is also open for exploring how surface charge and/or structural organization of water in the vicinity of a surface can affect the ability of the reacting species to adsorb (desorb). For example, we can address if the change in silica hydration reverse the cation adsorption strength [40,85]; or if the facet-specific hydration of particles can enable facet selective adsorption of proteins and transfer of electrons [86,87].

\subsection{Conclusion}

In the present work, using AFM force spectroscopy and tip size of $\approx 9 \mathrm{~nm}$ we simultaneously map hydration forces and DLVO forces of gibbsite nanoparticle and silica substrate in solutions of $\mathrm{pH}_{4}, 6,9$ and 10 and $100 \mathrm{mM} \mathrm{NaCl}$ and show that the oscillatory hydration structure and surface charge of materials are not correlated and respond very differently to a variation in the fluid composition. Analysis of the EDL force shows that silica is negatively charged that monotonically increases with $\mathrm{pH}$ (from $\mathrm{pH}_{4}$ to 9). The gibbsite basal plane has a heterogeneous positive charge that decreases with increasing $\mathrm{pH}$. The hydration force on silica and gibbsite is composed of an oscillatory force superimposed on to a monotonically decaying background force. The monotonic hydration force in all fluid compositions is attractive on gibbsite and repulsive on silica. The measured oscillatory hydration force profiles demonstrate that there are three organized water layers next to gibbsite nanoparticles and silica surface. The hydration layers on crystalline gibbsite surface are more organized than the ones observed on an amorphous silica surface as evidenced by amplitude and periodicity oscillations in the force. On the gibbsite basal plane, the strength of the structural hydration forces is highest at $\mathrm{pH}$ 6. On the amorphous silica surface, the oscillatory hydration forces are present only on $\approx 10 \%$ of the sample (on 
crystalline gibbsite on $\approx 60 \%$ ) and their strength is rather independent of fluid composition.

\subsection{Appendix}
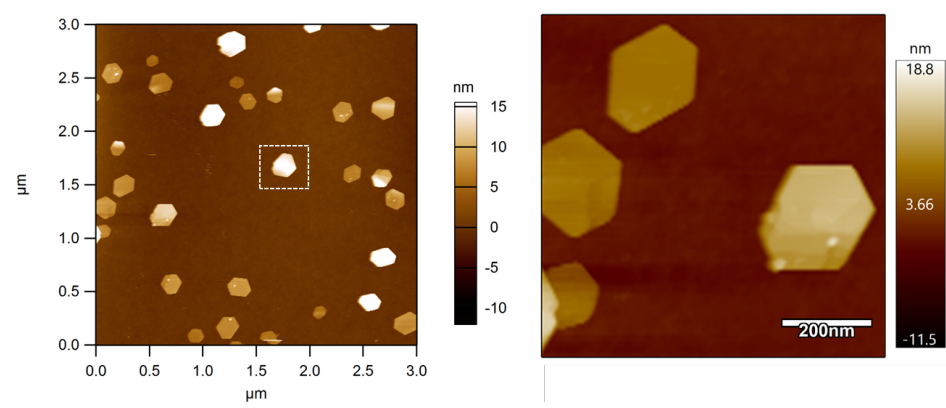

Figure 5.7: AM-AFM topography image of several gibbsite particles adsorbed on silica in a $10 \mathrm{mM} \mathrm{NaCl}$ solution at $\mathrm{pH}$ 6. Topography image shows the presence of smooth terraces and regions with defects on the gibbsite particles basal plane.

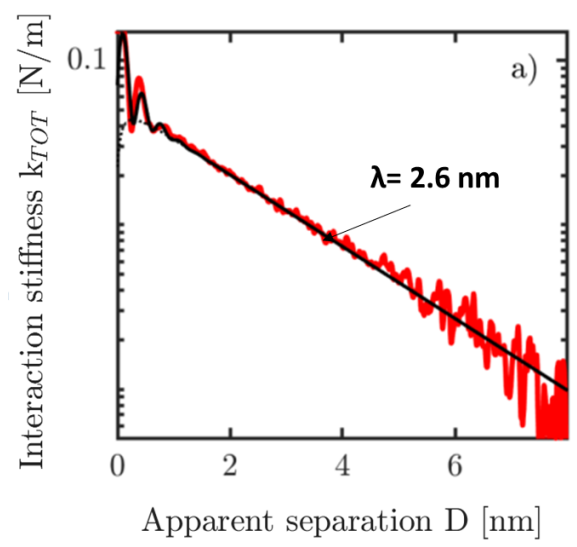

Figure 5.8: Decay length of the EDL force in a $10 \mathrm{mM} \mathrm{NaCl} \mathrm{pH} 6$ solution. 


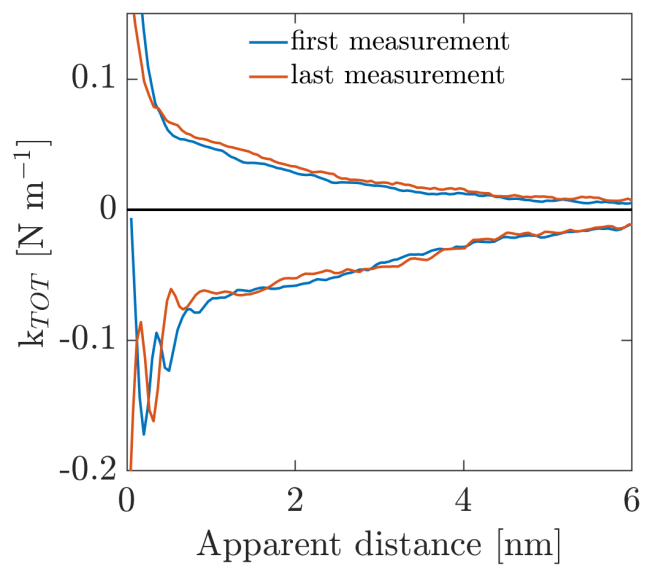

Figure 5.9: Interaction stiffness vs tip sample separation on silica and gibbsite at $10 \mathrm{mM}$ $\mathrm{NaCl}$, and $\mathrm{pH}$ 6, before and after completion of the $3 \mathrm{D}$ force maps at $\mathrm{pH} 4,6,9$ with 10 and $100 \mathrm{mM} \mathrm{NaCl}$.

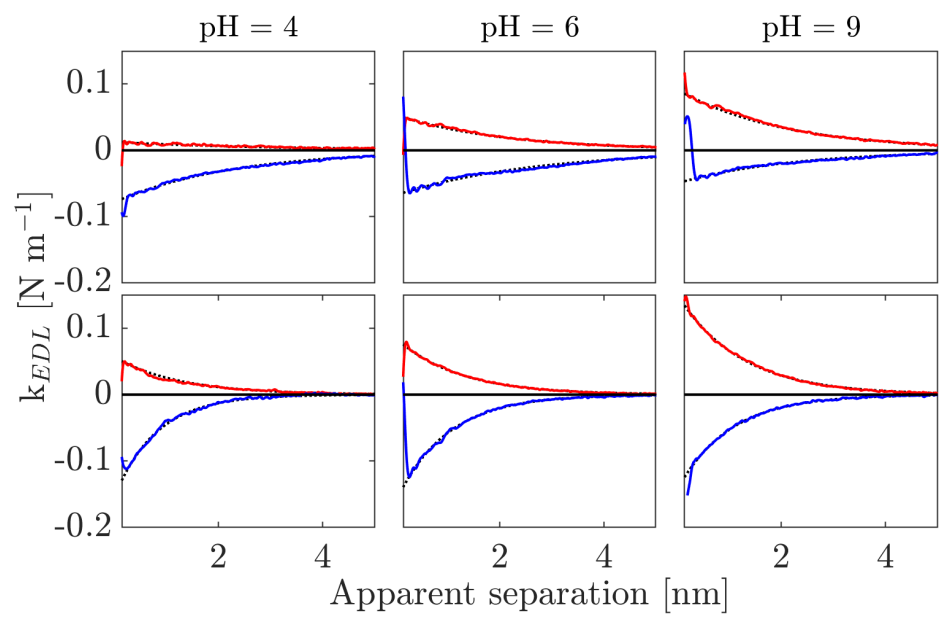

Figure 5.10: The EDL force gradient (total interaction force gradient with the hydration force gradient subtracted) as function of fluid composition for silica (red) and gibbsite (blue). Top row is $10 \mathrm{mM} \mathrm{NaCl}$ and bottom row is $100 \mathrm{mM} \mathrm{NaCl}$. 


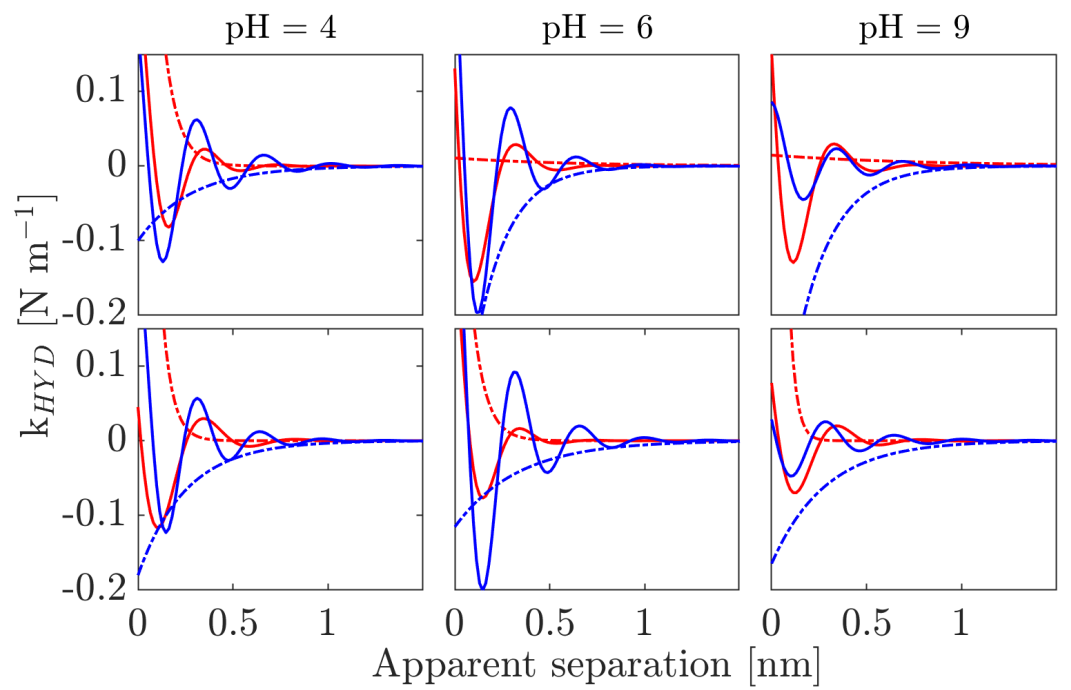

Figure 5.11: The structural (solid lines) and monotonic (dashed lines) hydration force gradient as a function of fluid composition generated using the values in table 5.2 for silica (red) and gibbsite (blue). Top row is $10 \mathrm{mM} \mathrm{NaCl}$ and bottom row is $100 \mathrm{mM} \mathrm{NaCl}$. 


\section{Bibliography}

[1] O. Bjorneholm, M. H. Hansen, A. Hodgson, L. M. Liu, D. T. Limmer, A. Michaelides, P. Pedevilla, J. Rossmeisl, H. Shen, G. Tocci, E. Tyrode, M. M. Walz, J. Werner, and H. Bluhm, "Water at interfaces," Chem Rev, vol. 1 16, no. 13, pp. 7698-726, 2016.

[2] J. Israelachvili and H. Wennerström, "Role of hydration and water structure in biological and colloidal interactions," Nature, vol. 379, no. 6562, pp. 219-225, 1996.

[3] C. Tournassat, I. C. Bourg, C. I. Steefel, and F. Bergaya, Surface Properties of Clay Minerals, pp. 5-31. Developments in Clay Science, Elsevier, 2015.

[4] G. Sposito, N. T. Skipper, R. Sutton, S.-h. Park, A. K. Soper, and J. A. Greathouse, "Surface geochemistry of the clay minerals," Proc Natl Acad Sci US A, vol. 96, no. 7, pp. 3358-3364, 1999.

[5] B. Bera, N. Kumar, M. H. G. Duits, M. A. Cohen Stuart, and F. Mugele, "Cationic hofmeister series of wettability alteration in mica-water-alkane systems," Langmuir, vol. 34, no. 45, pp. 13574-13583, 2018.

[6] F. Mugele, B. Bera, A. Cavalli, I. Siretanu, A. Maestro, M. Duits, M. CohenStuart, D. van den Ende, I. Stocker, and I. Collins, "Ion adsorption-induced wetting transition in oil-water-mineral systems," Sci Rep, vol. 5, p. $10519,2015$.

[7] A. Klaassen, F. Liu, D. van den Ende, F. Mugele, and I. Siretanu, "Impact of surface defects on the surface charge of gibbsite nanoparticles," Nanoscale, 2017.

[8] I. Siretanu, D. Ebeling, M. P. Andersson, S. L. Stipp, A. Philipse, M. C. Stuart, D. van den Ende, and F. Mugele, "Direct observation of ionic structure at solidliquid interfaces: a deep look into the stern layer," Sci Rep, vol. 4, p. 4956, 2014.

[9] N. Kumar, M. P. Andersson, D. van den Ende, F. Mugele, and I. Siretanu, "Probing the surface charge on the basal planes of kaolinite particles with highresolution atomic force microscopy," Langmuir, vol. 33, no. 50, pp. 14226$14237,2017$. 
[10] T. Preocanin, A. Abdelmonem, G. Montavon, and J. Luetzenkirchen, "Charging behavior of clays and clay minerals in aqueous electrolyte solutions experimental methods for measuring the charge and interpreting the results," 2016.

[11] K. C. Jena and D. K. Hore, "Variation of ionic strength reveals the interfacial water structure at a charged mineral surface," The Journal of Physical Chemistry C, vol. 113, no. 34, pp. 15364-15372, 2009.

[12] J. D. Cyran, M. A. Donovan, D. Vollmer, F. Siro Brigiano, S. Pezzotti, D. R. Galimberti, M. P. Gaigeot, M. Bonn, and E. H. G. Backus, "Molecular hydrophobicity at a macroscopically hydrophilic surface," Proc Natl Acad Sci U $S$ A, vol. 116, no. 5, pp. 1520-1525, 2019.

[13] A. Marchioro, M. Bischoff, C. Lütgebaucks, D. Biriukov, M. Předota, and S. Roke, "Surface characterization of colloidal silica nanoparticles by second harmonic scattering: Quantifying the surface potential and interfacial water order," The Journal of Physical Chemistry C, vol. 123, no. 33, pp. 20393-20404, 2019.

[14] P. Fenter and N. C. Sturchio, "Mineral-water interfacial structures revealed by synchrotron x-ray scattering," Progress in Surface Science, vol. 77, no. 5-8, pp. $171-258,2004$.

[15] J. Daillant and A. Gibaud, X-ray and Neutron Reflectivity: Principles and Applications. Springer, 2008.

[16] S. J. T. Brugman, B. L. Werkhoven, E. R. Townsend, P. Accordini, R. van Roij, and E. Vlieg, "Monovalent - divalent cation competition at the muscovite mica surface: Experiment and theory," J Colloid Interface Sci, vol. 559, pp. 291-303, 2020.

[17] J.-J. Velasco-Velez, C. H. Wu, T. A. Pascal, L. F. Wan, J. Guo, D. Prendergast, and M. Salmeron, "The structure of interfacial water on gold electrodes studied by x-ray absorption spectroscopy," Science, vol. 346, no. 6211, p. 831, 2014.

[18] L. N. Lammers, I. C. Bourg, M. Okumura, K. Kolluri, G. Sposito, and M. Machida, "Molecular dynamics simulations of cesium adsorption on illite nanoparticles," J Colloid Interface Sci, vol. 490, pp. 608-620, 2017. 
[19] C. Tournassat, J. A. Davis, C. Chiaberge, S. Grangeon, and I. C. Bourg, "Modeling the acid-base properties of montmorillonite edge surfaces," Environ Sci Technol, vol. 50, no. 24, pp. 13436-13445, 2016.

[20] A. Newton, K. Kwon, and D.-K. Cheong, "Edge structure of montmorillonite from atomistic simulations," Minerals, vol. 6, no. 2, 2016.

[21] M. Pouvreau, J. A. Greathouse, R. T. Cygan, and A. G. Kalinichev, "Structure of hydrated gibbsite and brucite edge surfaces: Dft results and further development of the clayff classical force field with metal-o-h angle bending terms," The Journal of Physical Chemistry C, vol. 121, no. 27, pp. 14757-14771, 2017.

[22] T. A. Ho, J. A. Greathouse, Y. Wang, and L. J. Criscenti, "Atomistic structure of mineral nano-aggregates from simulated compaction and dewatering," Sci Rep, vol. 7, no. 1, p. 15286, 2017.

[23] I. Siretanu, D. van den Ende, and F. Mugele, "Atomic structure and surface defects at mineral-water interfaces probed by in situ atomic force microscopy," Nanoscale, vol. 8, no. 15, pp. 8220-7, 2016.

[24] C. L. Zhao, D. Ebeling, I. Siretanu, D. van den Ende, and F. Mugele, "Extracting local surface charges and charge regulation behavior from atomic force microscopy measurements at heterogeneous solid-electrolyte interfaces," Nanoscale, vol. 7, no. 39, pp. 16298-16311, 2015.

[25] K. Umeda, L. Zivanovic, K. Kobayashi, J. Ritala, H. Kominami, P. Spijker, A. S. Foster, and H. Yamada, "Atomic-resolution three-dimensional hydration structures on a heterogeneously charged surface," Nat Commun, vol. 8, no. 1, p. 211 , 2017.

[26] T. Fukuma, Y. Ueda, S. Yoshioka, and H. Asakawa, "Atomic-scale distribution of water molecules at the mica-water interface visualized by three-dimensional scanning force microscopy," Physical Review Letters, vol. 104, no. 1, 2010.

[27] K. Kuchuk and U. Sivan, "Hydration structure of a single dna molecule revealed by frequency-modulation atomic force microscopy," Nano Lett, vol. 18, no. 4, pp. 2733-2737, 2018. 
[28] D. Martin-Jimenez, E. Chacon, P. Tarazona, and R. Garcia, "Atomically resolved three-dimensional structures of electrolyte aqueous solutions near a solid surface," Nat Commun, vol. 7, p. 12164, 2016.

[29] E. T. Herruzo, H. Asakawa, T. Fukuma, and R. Garcia, "Three-dimensional quantitative force maps in liquid with 10 piconewton, angstrom and subminute resolutions," Nanoscale, vol. 5, no. 7, pp. 2678-2685, 2013.

[30] C. Marutschke, D. Walters, J. Cleveland, I. Hermes, R. Bechstein, and A. Kühnle, "Three-dimensional hydration layer mapping on the (10.4) surface of calcite using amplitude modulation atomic force microscopy," Nanotechnology, vol. 25, no. 33, p. 335703, 2014.

[31] H. Songen, B. Reischl, K. Miyata, R. Bechstein, P. Raiteri, A. L. Rohl, J. D. Gale, T. Fukuma, and A. Kuhnle, "Resolving point defects in the hydration structure of calcite (10.4) with three-dimensional atomic force microscopy," Phys Rev Lett, vol. 120, no. 11, p. 116101, 2018.

[32] K. Miyazawa, N. Kobayashi, M. Watkins, A. L. Shluger, K. Amano, and T. Fukuma, "A relationship between three-dimensional surface hydration structures and force distribution measured by atomic force microscopy," Nanoscale, vol. 8, no. 13, pp. 7334-42, 2016.

[33] T. Fukuma, B. Reischl, N. Kobayashi, P. Spijker, F. F. Canova, K. Miyazawa, and A. S. Foster, "Mechanism of atomic force microscopy imaging of threedimensional hydration structures at a solid-liquid interface," Physical Review B, vol. 92, no. 15, 2015.

[34] T. Fukuma and R. Garcia, "Atomic- and molecular-resolution mapping of solidliquid interfaces by $3 \mathrm{~d}$ atomic force microscopy," ACS Nano, vol. 12, no. 12, pp. 11785-11797, 2018.

[35] A. M. Wierenga, T. A. J. Lenstra, and A. P. Philipse, "Aqueous dispersions of colloidal gibbsite platelets: synthesis, characterisation and intrinsic viscosity measurements," Colloids and Surfaces a-Physicochemical and Engineering Aspects, vol. 134, no. 3, pp. 359-371, 1998. 
[36] S. Louaer, Y. Wang, and L. Guo, "Fast synthesis and size control of gibbsite nanoplatelets, their pseudomorphic dehydroxylation, and efficient dye adsorption," ACS Appl Mater Interfaces, vol. 5, no. 19, pp. 9648-55, 2013.

[37] C. Sweegers, W. J. P. van Enckevort, H. Meekes, P. Bennema, I. D. K. Hiralal, and A. Rijkeboer, "The impact of twinning on the morphology of gammaal(oh)(3) crystals," Journal of Crystal Growth, vol. 197, no. 1-2, pp. 244-253, 1999.

[38] C. K. Kumara, W. J. Ng, A. Bandara, and R. Weerasooriya, "Nanogibbsite: synthesis and characterization," J Colloid Interface Sci, vol. 352, no. 2, pp. 252-8, 2010.

[39] D. M. Moore and R. C. Reynolds, X-ray diffraction and the identification and analysis of clay minerals. Oxford [England]; New York: Oxford University Press, 1989.

[40] M. Dishon, O. Zohar, and U. Sivan, "From repulsion to attraction and back to repulsion: The effect of nacl, $\mathrm{kcl}$, and cscl on the force between silica surfaces in aqueous solution," Langmuir, vol. 25, no. 5, pp. 2831-2836, 2009.

[41] A. Labuda, J. Cleveland, N. A. Geisse, M. Kocun, B. Ohler, R. Proksch, M. B. Viani, and D. Walters, "Photothermal excitation for improved cantilever drive performance in tapping mode atomic force microscopy," Microscopy and Analysis28, vol. 3, pp. $\mathrm{S}_{21}-\mathrm{S}_{25}, 2014$.

[42] N. Kumar, C. Zhao, A. Klaassen, D. van den Ende, F. Mugele, and I. Siretanu, "Characterization of the surface charge distribution on kaolinite particles using high resolution atomic force microscopy," Geochimica et Cosmochimica Acta, vol. 175, pp. 100-112, 2016.

[43] B. W. Ninham and Parsegia.Va, "Electrostatic potential between sufraces bearing ionizable groups in ionic equilibrium with physiologic saline solution," Journal of Theoretical Biology, vol. 31, no. 3, p. 405, 1971.

[44] S. H. Behrens and M. Borkovec, "Exact poisson-boltzmann solution for the interaction of dissimilar charge-regulating surfaces," Phys Rev E Stat Phys Plasmas Fluids Relat Interdiscip Topics, vol. 6o, no. 6 Pt B, pp. 7040-8, 1999. 
[45] S. H. Behrens and M. Borkovec, "Electric double layer interaction of ionizable surfaces: Charge regulation for arbitrary potentials," Journal of Chemical Physics, vol. 111, no. 1, pp. 382-385, 1999.

[46] Y. Gan and G. V. Franks, "Charging behavior of the gibbsite basal (oo1) surface in nacl solution investigated by afm colloidal probe technique," Langmuir, vol. 22, no. 14, pp. 6087-6092, 2006.

[47] H. J. Butt, B. Cappella, and M. Kappl, "Force measurements with the atomic force microscope: Technique, interpretation and applications," Surface Science Reports, vol. 59, no. 1-6, pp. 1-152, 2005.

[48] T. Hiemstra, W. Van Riemsdijk, and G. Bolt, "Multisite proton adsorption modeling at the solid/solution interface of (hydr) oxides: A new approach: I. model description and evaluation of intrinsic reaction constants," Journal of colloid and interface science, vol. 133, no. 1, pp. 91-104, 1989.

[49] S. R.van Lin, K. K. Grotz, I. Siretanu, N. Schwierz, and F. Mugele, "Ion-specific and ph-dependent hydration of mica-electrolyte interfaces," Langmuir, vol. 35, no. 17, pp. 5737-5745, 2019.

[50] J. N. Israelachvili and R. M. Pashley, "Molecular layering of water at surfaces and origin of repulsive hydration forces," Nature, vol. 306, no. 5940, pp. 249250,1983 .

[51] J. I. Kilpatrick, S. H. Loh, and S. P. Jarvis, "Directly probing the effects of ions on hydration forces at interfaces," Journal of the American Chemical Society, vol. 135, no. 7, pp. 2628-2634, 2013.

[52] U. Raviv and J. Klein, "Fluidity of bound hydration layers," Science, vol. 297, no. 5586, pp. 1540-3, 2002.

[53] M. Ricci, P. Spijker, F. Stellacci, J. F. Molinari, and K. Voitchovsky, "Direct visualization of single ions in the stern layer of calcite," Langmuir, vol. 29, no. 7, pp. 2207-2216, 2013.

[54] D. Argyris, T. Ho, D. R. Cole, and A. Striolo, "Molecular dynamics studies of interfacial water at the alumina surface," The Journal of Physical Chemistry $C$, vol. 115, no. 5, pp. 2038-2046, 2011. 
[55] D. L. Patrick and R. M. Lynden-Bell, "Atomistic simulations of fluid structure and solvation forces in atomic force microscopy," Surface Science, vol. 380, no. 2, pp. 224-244, 1997.

[56] R. Ho, J.-Y. Yuan, and Z. Shao, "Hydration force in the atomic force microscope: A computational study," Biophysical Journal, vol. 75, no. 2, pp. 1076$1083,1998$.

[57] D. Argyris, A. Phan, A. Striolo, and P. D. Ashby, "Hydration structure at the alpha-al203 (0001) surface: Insights from experimental atomic force spectroscopic data and atomistic molecular dynamics simulations," Journal of Physical Chemistry C, vol. 117, no. 20, pp. 10433-10444, 2013.

[58] T. Baimpos, B. R. Shrestha, S. Raman, and M. Valtiner, "Effect of interfacial ion structuring on range and magnitude of electric double layer, hydration, and adhesive interactions between mica surfaces in 0.05-3 $\mathrm{m} \mathrm{li}(+)$ and cs $(+)$ electrolyte solutions," Langmuir, vol. 30, no. 15, pp. 4322-32, 2014.

[59] J. P. Chapel, "Electrolyte species dependent hydration forces between silica surfaces," Langmuir, vol. 10, no. 11, pp. 4237-4243, 1994.

[6o] R. M. Pashley, "Hydration forces between mica surfaces in aqueous electrolyte solutions," Journal of Colloid and Interface Science, vol. 80, no. 1, pp. 153-162, 1981.

[61] H. J. Butt, "Measuring electrostatic, vanderwaals, and hydration forces in electrolyte-solutions with an atomic force microscope," Biophysical Journal, vol. 6o, no. 6, pp. 1438-1444, 1991.

[62] R. R. Netz, "Static van der waals interactions in electrolytes," The European Physical Journal E, vol. 5, no. 2, pp. 189-205, 2001.

[63] S. Kuhn and P. Rahe, "Discriminating short-range from van der waals forces using total force data in noncontact atomic force microscopy," Physical Review $B$, vol. 89 , no. 23,2014 .

[64] A. Striolo, "From interfacial water to macroscopic observables: A review," Adsorption Science \& Technology, vol. 29, no. 3, pp. 211-258, 2011. 
[65] J. G. Catalano, P. Fenter, and C. Park, "Interfacial water structure on the (012) surface of hematite: Ordering and reactivity in comparison with corundum," Geochimica et Cosmochimica Acta, vol. 71, no. 22, pp. 5313-5324, 2007.

[66] J. Wang, A. G. Kalinichev, and R. J. Kirkpatrick, "Effects of substrate structure and composition on the structure, dynamics, and energetics of water at mineral surfaces: A molecular dynamics modeling study," Geochimica et Cosmochimica Acta, vol. 70, no. 3, pp. 562-582, 2006.

[67] T. A. Ho, J. A. Greathouse, A. S. Lee, and L. J. Criscenti, "Enhanced ion adsorption on mineral nanoparticles," Langmuir, vol. 34, no. 20, pp. 5926-5934, 2018.

[68] B. R. Bickmore, C. J. Tadanier, K. M. Rosso, W. D. Monn, and D. L. Eggett, "Bond-valence methods for $\mathrm{pk}(\mathrm{a})$ prediction: critical reanalysis and a new approach," Geochimica Et Cosmochimica Acta, vol. 68, no. 9, pp. 2025-2042, 2004.

[69] B. R. Bickmore, K. M. Rosso, C. J. Tadanier, E. J. Bylaska, and D. Doud, "Bondvalence methods for $\mathrm{pk}(\mathrm{a})$ prediction. ii. bond-valence, electrostatic, molecular geometry, and solvation effects," Geochimica Et Cosmochimica Acta, vol. 70, no. 16, pp. 4057-4071, 2006.

[70] J. J. Valle-Delgado, J. A. Molina-Bolivar, F. Galisteo-Gonzalez, M. J. GalvezRuiz, A. Feiler, and M. W. Rutland, "Hydration forces between silica surfaces: Experimental data and predictions from different theories," Journal of Chemical Physics, vol. 123, no. 3, 2005.

[71] G. Vigil, Z. Xu, S. Steinberg, and J. Israelachvili, "Interactions of silica surfaces," pp. Medium: X; Size: Pages: $367-385,1994$.

[72] R. G. Horn, D. T. Smith, and W. Haller, "Surface forces and viscosity of water measured between silica sheets," Chemical Physics Letters, vol. 162, no. 4, pp. 404-408, 1989.

[73] W. A. Ducker, T. J. Senden, and R. M. Pashley, "Measurement of forces in liquids using a force microscope," Langmuir, vol. 8, no. 7, pp. 1831-1836, 1992. 
[74] W. A. Ducker, T. J. Senden, and R. M. Pashley, "Direct measurement of colloidal forces using an atomic force microscope," Nature, vol. 353, no. 6341, pp. 239-241, 1991.

[75] A. Grabbe and R. G. Horn, "Double-layer and hydration forces measured between silica sheets subjected to various surface treatments," Journal of Colloid and Interface Science, vol. 157, no. 2, pp. 375-383, 1993.

[76] B. M. Lowe, Y. Maekawa, Y. Shibuta, T. Sakata, C. K. Skylaris, and N. G. Green, "Dynamic behaviour of the silica-water-bio electrical double layer in the presence of a divalent electrolyte," Phys Chem Chem Phys, vol. 19, no. 4, pp. 26872701, 2017.

[77] R. West, L. S. Whatley, and K. J. Lake, "Hydrogen bonding studies. v. the relative basicities of ethers, alkoxysilanes and siloxanes and the nature of the silicon-oxygen bond 1,2," Journal of the American Chemical Society, vol. 83, no. 4, pp. 761-764, 1961.

[78] J. Rogelj, D. Shindell, K. Jiang, S. Fifita, P. Forster, V. Ginzburg, C. Handa, H. Kheshgi, S. Kobayashi, E. Kriegler, L. Mundaca, R. Seferian, M. Vilarino, K. Calvin, O. Edelenbosch, J. Emmerling, S. Fuss, T. Gasser, N. Gillet, C. He, E. Hertwich, L. Höglund Isaksson, D. Huppmann, G. Luderer, A. Markandya, D. McCollum, R. Millar, M. Meinshausen, A. Popp, J. Pereira, P. Purohit, K. Riahi, A. Ribes, H. Saunders, C. Schadel, C. Smith, P. Smith, E. Trutnevyte, Y. Xiu, K. Zickfeld, and W. Zhou, Chapter 2: Mitigation pathways compatible with $1.5^{\circ} \mathrm{C}$ in the context of sustainable development. Intergovernmental Panel on Climate Change, 2018.

[79] A. M. Schrader, J. I. Monroe, R. Sheil, H. A. Dobbs, T. J. Keller, Y. Li, S. Jain, M. S. Shell, J. N. Israelachvili, and S. Han, "Surface chemical heterogeneity modulates silica surface hydration," Proceedings of the National Academy of Sciences, vol. 115 , no. 12, p. $2890,2018$.

[8o] I. S. Chuang and G. E. Maciel, "Probing hydrogen bonding and the local environment of silanols on silica surfaces via nuclear spin cross polarization dynamics," Journal of the American Chemical Society, vol. 118, pp. 401-406, 1996. 
[81] S. Marčelja and N. Radić, "Repulsion of interfaces due to boundary water," Chemical Physics Letters, vol. 42, no. 1, pp. 129-130, 1976.

[82] N. Radić and S. Marčelja, "Solvent contribution to the debye screening length," Chemical Physics Letters, vol. 55, no. 2, pp. 377-379, 1978.

[83] M. Kanduc, A. Schlaich, E. Schneck, and R. R. Netz, "Water-mediated interactions between hydrophilic and hydrophobic surfaces," Langmuir, vol. 32, no. 35 , pp. $8767-82,2016$.

[84] J. Addai-Mensah, J. Dawe, R. Hayes, C. Prestidge, and J. Ralston, "The unusual colloid stability of gibbsite at high ph," Journal of Colloid and Interface Science, vol. 203, no. 1, pp. 115-121, 1998.

[85] J. Morag, M. Dishon, and U. Sivan, "The governing role of surface hydration in ion specific adsorption to silica: an afm-based account of the hofmeister universality and its reversal," Langmuir, vol. 29, no. 21, pp. 6317-22, 2013.

[86] L. Dong, Q. Luo, K. Cheng, H. Shi, Q. Wang, W. Weng, and W. Q. Han, "Facet-specific assembly of proteins on srtio(3) polyhedral nanocrystals," Sci Rep, vol. 4, p. 5084, 2014.

[87] C. Y. Chiu, Y. Li, L. Ruan, X. Ye, C. B. Murray, and Y. Huang, "Platinum nanocrystals selectively shaped using facet-specific peptide sequences," Nat Chem, vol. 3, no. 5, pp. 393-9, 2011. 


\section{6}

\section{$\mathrm{pH}$ and divalent ion dependence of gibbsite nanoparticles}

\subsection{Introduction}

Ion adsorption plays an important role in many chemical reactions in many areas of science. It plays a crucial role in the stability of colloidal suspensions, the retention of contaminants in soil, the stability of DNA molecules and the retention of oil in oil reservoirs ${ }^{[1-5]}$. In the latter, divalent ions form a bridge between the negatively charged clay surface and negatively charged organic molecules in the oil. For sandstone reservoirs, this is expected to be an important reason for a decrease in recovered oil.

To further understand the role of the divalent ion bridge in oil recovery, divalent ion adsorption on clay minerals was investigated. For example, using Atomic Force Microscopy (AFM) it was shown that divalent ions like $\mathrm{Ca}^{2+}$ and $\mathrm{Mg}^{2+}$ can adsorb onto the surface of gibbsite (clay mineral). There they can form a uniform layer of 
adsorbed ions [6]. The pattern visualized by AFM shows that a double row pattern appears at intermediate concentrations (below $10 \mathrm{mM} \mathrm{CaCl}_{2}$ ) and at higher concentrations a single row pattern appears. Together with DFT calculations it was found that the double row pattern that appeared consisted of hydrated calcium $\left(\mathrm{Ca}^{2+}\right)$ ions adsorbed onto the clay mineral lattice. The single row was shown to be an additional chloride ion that adsorbed on top of the double row. The double row consists of alternating bumps with a periodicity of $0.50 \mathrm{~nm}$ and $0.87 \mathrm{~nm}$ along and perpendicular to the double row. This means that there are two bumps per surface unit cell. The bumps of the single rows were one lattice vector along the $b$ direction $(0.51 \mathrm{~nm})$ and one per unit cell in the a direction (see image 1.1 for gibbsite lattice). Similar results were found when gibbsite was exposed to $\mathrm{MgCl}_{2}$ solutions.

The periodicity of the bumps is very close to the periodicities of the surface unit cell of gibbsite, suggesting that the adsorption occurs at specific surface sites and is not electrostatically correlated. When comparing the location of the bumps with respect to the gibbsite lattice, they are similar to the location of $\mathrm{OH}$-groups that are available for hydrogen bonding. Two bumps per unit cell lead to a hypothetical surface charge of $9.2 \mathrm{e} / \mathrm{nm}^{2}$, which is not reflected in the force spectroscopy measurements.

From density functional theory (DFT) calculations the same stable double row pattern on gibbsite could be reproduced. To guarantee charge neutrality, $(-\mathrm{OH})$ groups were added. Three of the four groups acted as hydrogen bonding acceptors for surface protons, whereas the fourth formed a bridge between two cations. This leads to the surface unit cell to be charge neutral (o e $/ \mathrm{nm}^{2}$ instead of $9.2 \mathrm{e} / \mathrm{nm}^{2}$ !). However, in the force spectroscopy measurements a small positive charge (in the order of $0.1 \mathrm{e} / \mathrm{nm}^{2}$ ) was measured. The DFT calculations showed that the alternating structure of hydrated calcium ions offers several sites where hydration water and hydroxide ions $\left(\mathrm{OH}^{-}\right)$can bridge two cations. Additional calculations for the $\mathrm{pKa}$ for $\mathrm{H}_{2} \mathrm{O} \longrightarrow \mathrm{OH}^{-}$suggested that this positive charge in the AFM experiments could result from the partial protonation of a hydroxide ion that bridges adjacent cations from the solution. The DFT calculations also showed that at a high $\mathrm{Ca}^{2+}$ concentration, one $\mathrm{Cl}^{-}$-ion replaces the bridging $\mathrm{OH}^{-}$-ion, disabling the partial protonation of the $\mathrm{OH}^{-}$, resulting in a neutral surface site. This effectively reduces the diffuse layer charge, which is corroborated by the force spectroscopy measurements. Since results from computer simulations/calculations depend on input parameters and boundary conditions, the question arises whether the conclusion about the in- 
volvement of the $\mathrm{OH}^{-}$is true.

From the calculations we can see that for a single surface unit cell on gibbsite four to five $\mathrm{OH}^{-}$groups/ions are involved. From all this $\mathrm{OH}^{-}$involvement a $\mathrm{pH}$ dependence is to be expected. So, by changing the $\mathrm{pH}$ the balance of the number of available hydroxide ions from the bulk solution can be changed, disturbing the stabilizing effect of the hydroxide ions on the adsorbed cations. Using force spectroscopy at different $\mathrm{pH}$ and salt concentrations, the findings from the DFT calculations can be verified. Force spectroscopy can reveal this, since the point at which the increasing diffuse layer charge on gibbsite switches to decreasing can be measured. Therefore, in this chapter force spectroscopy is performed to study the stabilizing effect of the hydroxide ion on the cation adsorption on clay minerals. We measure the diffuse layer charge of gibbsite nanoparticles and silica in various calcium chloride concentrations as a function of $\mathrm{pH}$. In addition, we also measure the effect of ion radius and chemical affinity by measuring the diffuse layer charge of different cation $\left(\mathrm{BrCl}_{2}\right)$ and anion $\left(\mathrm{Ca}\left(\mathrm{NO}_{3}\right)_{2}\right)$ species. The force spectroscopy proved to be more difficult than anticipated. Therefore, this chapter shows all results and discusses questions that arise from the measurements.

\subsection{Methods and Materials}

\subsubsection{Sample and probe preparation}

The sample and cantilever is cleaned following the procedure mentioned in chapter 4.2 .

For the measurements, rectangular cantilevers (MikroMash $\mathrm{NSC}_{3} 6 / \mathrm{Cr}$-Au BS) are used and cleaned following the procedure in chapter 3.2 .1 .

\subsubsection{Amplitude modulation force spectroscopy}

Dynamic force spectroscopy measurements were performed with a commercial Asylum Research Cypher ES equipped with photo thermal excitation. All experiments are performed following the procedure in chapter 4.2. Figure 2.12a shows an example 
of the amplitude and phase shift curves on silica and gibbsite. The interaction forces in divalent electrolyte solutions are small, while the tip is quite sharp $(\sim 5 \mathrm{~nm})$. To increase the interaction area of the tip, it is flattened by imaging 4 times a $500 \mathrm{~nm}^{2}$ area in contact mode $($ scan rate $=0.5 \mathrm{~Hz}$,set-point $=0.5 \mathrm{~V})$. This leads to a tip with an effective tip radius of $50 \mathrm{~nm}$ (see figure 6.1) and to a local parallel plate geometry with a small contribution from the adjacent cone (figure 6.1). Since gibbsite is more brittle than the silica tip, it is possible to damage the gibbsite during the force spectroscopy leading to tip contamination. To minimize the risk of contamination, we do not allow the amplitude signal to drop below a chosen threshold ( $60 \%$ of amplitude) of its free amplitude value $(\approx 1 \mathrm{~nm})$. This will prevent the tip from exerting a large force on the gibbsite.

\subsubsection{Averaging}

A large amount of data has been recorded, in an attempt to properly reveal the charge dependence on silica and gibbsite as function of cation type, ion concentration and $\mathrm{pH}$. Approximately, the interaction stiffness on silica and gibbsite was measured in 50 different electrolyte solutions $\left(\mathrm{CaCl}_{2}, \mathrm{BaCl}_{2}, \mathrm{CaNO}_{3}\right.$ and $\left.\mathrm{CaBr}_{2}\right)$ over 15 different experiments, resulting in around 500.000 individual approaches. To simplify the analysis, we choose to average a representative region in the force map (one on silica, one on gibbsite) and do the further analysis on these averages. An example is shown in figure 2.15. Here, a force map is shown with two marked areas (one on silica, one on gibbsite), and the resulting average force is shown in the graph next to it. The graph also shows the PCA cleaned data in red. The effectiveness of the PCA routine is now reduced, since averaging increases the signal to noise ratio by sacrificing lateral resolution. The size of the averaged area dependents on the experimental conditions. In this example, a $32 \times 32$ grid of forces was recorded over an area of 250 by $250 \mathrm{~nm}^{2}$ and an area of $8 \times 8$ pixels was averaged. Depending on the pixel density and recorded area size, an average area was calculated of 4 by 4 to 10 by 10 forces. The routines calculate the derivative of the static deflection too, which is the average position of the cantilever during approach and retract. As explained in section 3.2.5, calculating the derivative of the static deflection serves as a quality check of our data. The signal to noise ratio is worse than that for the amplitude modulation (that is why we use amplitude modulation) and therefore it will not be shown in this analysis. 

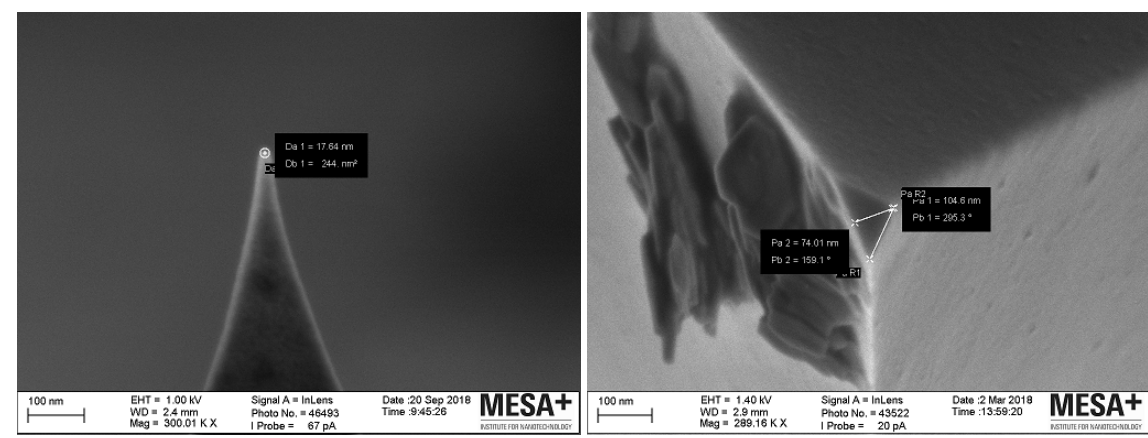

Figure 6.1: SEM images of the different types of tips used in the measurements. A sharp tip (left) and a blunted tip (right). Left tip parameters $R_{\text {tip }}=9 \mathrm{~nm}, \omega=22.3 \mathrm{kHz}$, $k=0.9 \mathrm{~N} \mathrm{~m}^{-1}, Q=3.5$. Right tip parameters $R_{\text {tip }}=48 \mathrm{~nm}, \omega=35.0 \mathrm{kHz}, k=$ 2.0 $\mathrm{N} \mathrm{m}^{-1}, Q=3.6$.

\subsubsection{Cantilevers}

Due to the large amount of measurements, the number of used cantilevers is also large. Therefore, an appropriate method is needed to compare all measurements. As explained in chapter 2 , the result from the inversion routine depends on the shape of the tip, e.g. blunted or sharp. When the amplitude and phase are converted, the tip geometry dictates whether the inversion routine (shown below and in chapter 2 as equation 2.9) yields the force or the interaction stiffness.

$$
k_{\text {int }}=k_{c}\left[1-\left(\omega / \omega_{\mathrm{o}}\right)^{2}\right] \frac{A_{\infty} \cos \left(\varphi-\varphi_{\infty}\right)-A}{A}+\gamma_{c} \omega \frac{A_{\infty} \sin \left(\varphi-\varphi_{\infty}\right)}{A}
$$

In the experiments, sharp and blunted cantilever tips were used, an example of used tips can be found in figure 6.1. To use both types of results in the analysis, the disjoining pressure is calculated. Therefore, in this chapter the disjoining pressure will be shown instead of the interaction force, as opposed to the other chapters. For a plate-sphere geometry, the data retrieved from the force inversion routine only has to be normalized by $2 \pi R$ (equation 2.15 ), whereas for a plate-plate geometry the data needs to be integrated and then normalized by $\pi R^{2}$ (equation 2.3.1). 


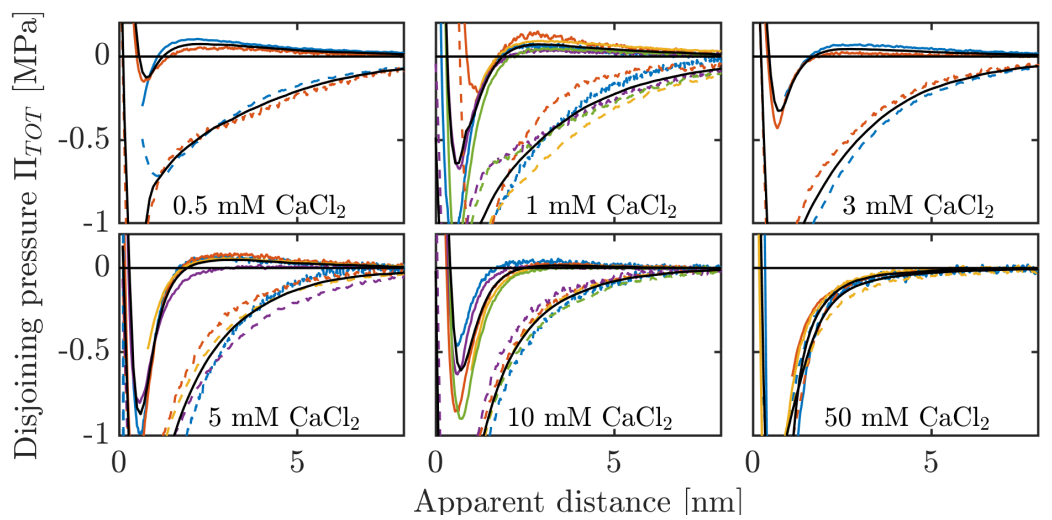

Figure 6.2: Disjoining pressure $\left(\Pi_{\text {total }}\right)$ versus distance curves on silica (solid curves) and gibbsite (dashed curves) in a series of $\mathrm{CaCl}_{2}$ solutions at $\mathrm{pH}$ 6. Different colors are different measurements, recorded with blunted and sharp tips. The graph of 0.5 and $3.0 \mathrm{mM}$ $\mathrm{CaCl}_{2}$ solution shows the disjoining pressure for a blunted tip (blue) and a sharp tip (red). The black line in the figure is the average of all the curves for the specific substrate. Tip parameters range over a wide range $R_{\text {tip }}=10-60 \mathrm{~nm}, \omega=430 \mathrm{kHz}, k=0.2-2.0 \mathrm{~N} \mathrm{~m}^{-1}$, $Q=1-3.5$.

\subsection{Results and discussion}

Figure 6.2 shows the disjoining pressure $\left(\Pi_{\text {total }}\right)$ versus the distance on silica and gibbsite in various $\mathrm{CaCl}_{2}$ solutions at $\mathrm{pH} 6$ for multiple experiments. The upper curves in the graphs are the silica-silica interactions, and the lower ones are the silicagibbsite interaction. At $50 \mathrm{mM}$ the silica and gibbsite curves have collapsed and the difference is hardly visible.

As is obvious, the results are not identical. Despite the large variety of used cantilever types, i.e. difference in tip radius, cantilever spring constant, resonance frequency and quality factor, the results show an acceptable consistency, especially above $1 \mathrm{~nm}$. Below $1 \mathrm{~nm}$ significant deviations appear, where most likely differences in the tip geometry cause a difference in the short range repulsion force. Figure 6.2 shows that for increasing calcium ion concentration, the repulsive force on silica decreases. At $50 \mathrm{mM}$ the force does not show any repulsive character, but is completely attractive. 


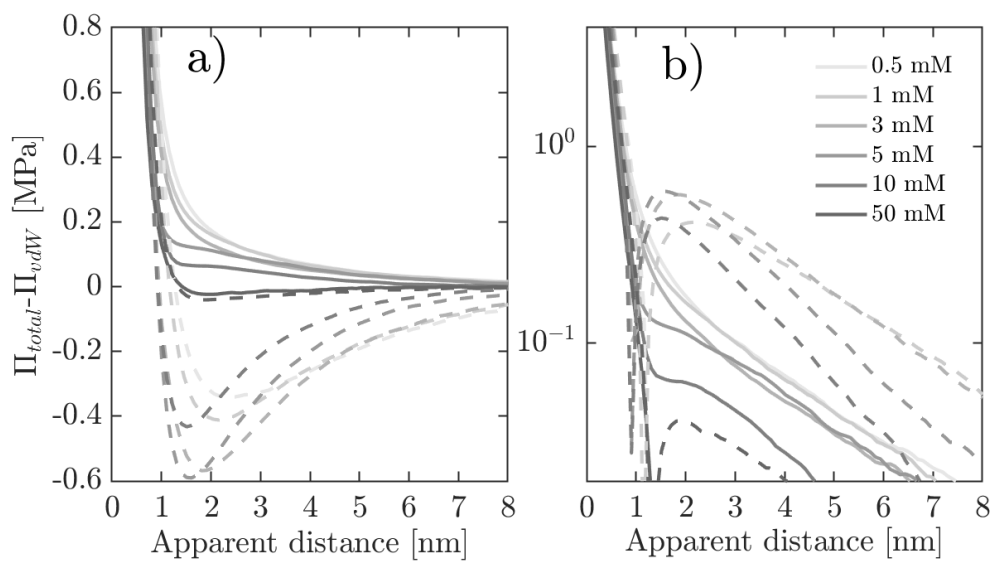

Figure 6.3: The disjoining pressure a) $\left(\Pi_{\text {total }}-\Pi_{v d W}\right)$ and b) $\left(\left|\Pi_{\text {total }}-\Pi_{v d W}\right|\right)$ on silica (solid lines) and gibbsite (dashed lines) averaged from the data in figure 6.2. Darkness of lines increases for increasing concentration.

At this point the disjoining pressure is expected to consist of only short range forces (like van der Waals force), since the electrostatic pressure has been screened completely. The decay length of the pressure decreases for increasing salt concentration, which is expected.

To examine how the disjoining pressure varies with $\mathrm{CaCl}_{2}$ concentration on silica and gibbsite, we take the averages determined in figure 6.2, remove the van der Waals contribution $\left(\Pi_{v d W}\right)$ using equation 2.12 with $R=10 \mathrm{~nm}, A_{H s i l}=0.65 \times 10^{-20} \mathrm{~J}$ and $A_{H g i b}=1.3 \times 10^{-20} \mathrm{~J}$ and display it in figure 6.3. Here, the disjoining pressure $\left(\Pi_{\text {total }}-\Pi_{v d W}\right)$ is shown for silica and gibbsite in solid and dashed gray lines, respectively. In figure $6.3 \mathrm{~b}$ the absolute disjoining pressure $\left(\left|\Pi_{\text {total }}-\Pi_{v d W}\right|\right)$ is plotted in a semi log scale. For silica the repulsive disjoining pressure decreases for increasing salt concentration. Above $10 \mathrm{mM} \mathrm{CaCl}{ }_{2}$ the pressure is almost zero. For gibbsite the same trend is observed, where an increasing salt concentration results in a decrease in (attractive) pressure. At $50 \mathrm{mM}$ the pressure on both silica and gibbsite is almost completely screened. Note that, the disjoining pressure measured for gibbsite depends on the charge of the silica cantilever tip. This means that the $\mathrm{pH}$ and salt concentration effects seen for the gibbsite data contains the $\mathrm{pH}$ and salt concen- 


\begin{tabular}{ccccccc} 
Expected $\kappa^{-1}$ & $7.8(0.5)$ & $5.5(1)$ & $3.2(3)$ & $2.5(5)$ & $1.7(10)$ & $0.8(50)$ \\
\hline pH 4 & & 2.8 & & 2.3 & 1.7 & 0.3 \\
pH 6 & 2.9 & 2.6 & 2.4 & 2.3 & 1.7 & 0.3 \\
pH 9 & & 2.8 & & 2.4 & 1.7 & 0.3
\end{tabular}

Table 6.1: Fitted decay lengths using $\kappa^{-1}=0.174 / \sqrt{I(M)}$. The expected ionic concentration in between brackets. Data fitted from figure 6.2 and 6.3 .

tration dependent charge trends of silica. We know from the trend on silica, that the charge on the tip is not constant. The qualitative trend on gibbsite is retrieved by converting the disjoining pressure to a diffuse layer charge.

Next, we take a look at the decay lengths of figure 6.3, which can be described using the Debye length. The Debye length for a monovalent aqueous solution can be approximated by $\kappa^{-1}(\mathrm{~nm})=0.304 / \sqrt{I(M)}$ for certain conditions. This means that for a $10 \mathrm{mM}$ monovalent salt, we expect a Debye length of $3 \mathrm{~nm}$, whereas for a $2: 1$ salt a Debye length of $\kappa^{-1}(\mathrm{~nm})=0.174 / \sqrt{I(M)}=1.7 \mathrm{~nm}$ is expected ${ }^{[8]}$. Judging from figure 6.3, this seems not to be the case. Almost all the decay lengths for the experiments are not of the expected size (table 6.1). However, the decay length does decrease for increasing salt concentration. Surprising, too, is that all solutions have been diluted from a $100 \mathrm{mM}$ stock solution, while $10 \mathrm{mM}$ solution seems to be the only solution with the expected Debye length. The forces from which the decay length can be fitted are very small and are very sensitive to the range over which these forces are fitted. The error in the fitted Debye lengths is significant, and increases for an increasing salt concentration, since the fitting range becomes shorter. Moreover, the Derjaguin approximation is only valid for a limited range $\left(R_{\text {tip }}>>z>>\kappa^{-1}\right)^{[9]}$, where $z$ is the tip-sample distance. At $1 \mathrm{mM}$ the error is up to $20 \%$, whereas at $50 \mathrm{mM}$ this increases up to $60 \%$. From the error analysis it becomes clear that the fitted values will not match the expected Debye lengths, within error.

The raw experimental data shows the unexpected decay lengths as well, and therefore the deviation is not a result of the force inversion routine. Also, the workflow does not deviate from the approach with monovalent solutions.

pH dependency Despite the deviations in the decay length, we investigate the sta- 


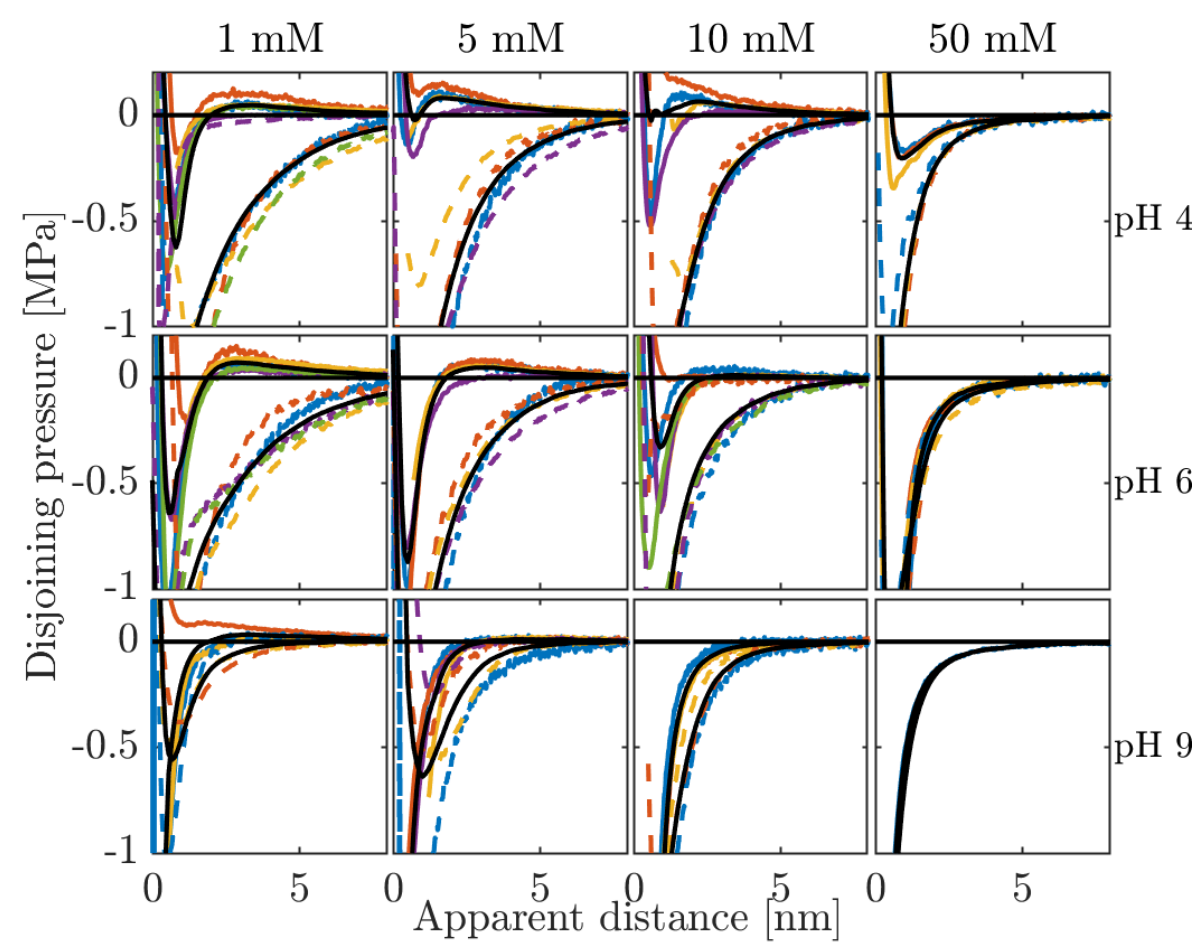

Figure 6.4: Disjoining pressure $\Pi_{T O T}$ as function of apparent tip-sample distance for various $\mathrm{pH}$ and $\mathrm{CaCl}_{2}$ concentrations on silica (solid curves) and gibbsite (dashed curves). Each colored curve is an average of 25 to 64 approaches. The black curves are the average of the measurements. 


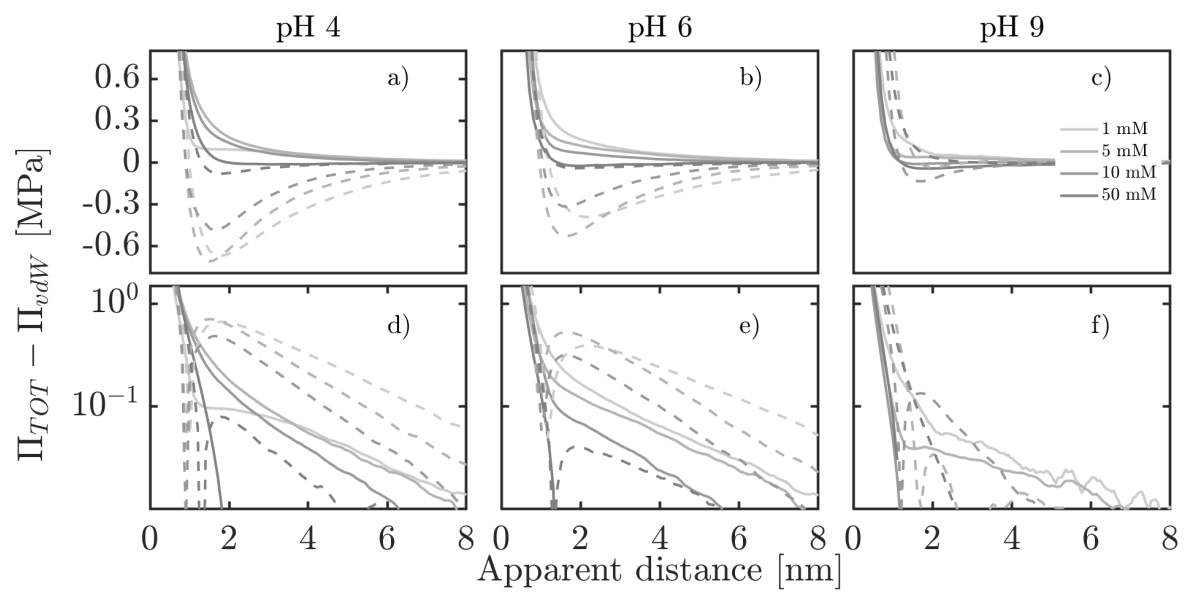

Figure 6.5: The disjoining pressure a) $\left(\Pi_{\text {total }}-\Pi_{v d W}\right)$ and b) $\left(\left|\Pi_{\text {total }}-\Pi_{v d W}\right|\right)$, taken from the black data in figure 6.4, as function of apparent tip-sample distance for various $\mathrm{CaCl}_{2}$ concentrations on silica (solid curves) and gibbsite (dashed curves) for $\mathrm{pH}_{4}, 6$ and 9.

bilizing role of the hydroxide $\left(\mathrm{OH}^{-}\right)$ions on the divalent cations. Changing the $\mathrm{pH}$ changes the balance of available $\mathrm{OH}^{-}$-ions, possibly changing the amount of adsorbed $\mathrm{Ca}^{2+}$-ions, thus changing the charge. In figure 6.4 results for $\mathrm{pH}$ dependent measurements are shown.

From the data in figure 6.4, the averages are taken and the van der Waals contribution is subtracted $\left(\Pi_{v d W}\right.$, using equation 2.12 with $\left.R=10 \mathrm{~nm}\right)$ and shown by $\mathrm{pH}$ in figure 6.5. The subtracted van der Waals contribution is based on the pressure at $50 \mathrm{mM} \mathrm{pH}$. Here, we assume that the electrostatic force is completely screened and only short range forces are left, like the van der Waals force. This contribution is subtracted for all conditions.

The trends observed for silica and gibbsite, at $\mathrm{pH} 4$ and 9 , are similar to that at $\mathrm{pH}$ 6 (decay lengths are noted in table 6.1). However, for $\mathrm{pH} 4$ the absolute pressures are larger, both for silica and gibbsite and for $\mathrm{pH} 9$ the absolute pressures are lower. The pressure on gibbsite switches from negative (attractive) to positive (repulsive) at high salt concentrations at $\mathrm{pH}$ 9. At these conditions, the approaches on gibbsite and silica overlap as can be seen in figure 6.4. Since the van der Waals contribution is 
larger for gibbsite $\left(A_{H}=13 \cdot 10^{-21} \mathrm{~J}\right)$ than for silica $\left(A_{H}=6.5 \cdot 10^{-21} \mathrm{~J}\right)$, the resulting pressure changes to positive on gibbsite. Also, the repulsive disjoining pressure for silica is screened at lower concentrations for increasing $\mathrm{pH}$. At very high values, the data converges to a zero apparent distance.

The idea of averaging over large datasets was to overcome one of the downsides of AFM, which is the statistical disadvantage of data generation. It is quite difficult to generate enough data to get statistically relevant results. Therefore, I included a large amount of data in the analysis of the results presented in this chapter. However, the large dataset does not seem to aid the visibility of trends, but rather obscure them. The trends found in figure 6.6 are clearer than the ones in figure 6.5, whereas figure 6.6 consist of single experiments only. One reason for the reduced effectiveness of averaging is that the experiments used for the averaging cover a large amount of cantilever types with different characteristics. Moreover, all experiments are performed in freshly prepared solutions that might have slight variations in the concentration. Also, for every experiment a new gibbsite nanoparticle is used for the force spectroscopy. All these slight variations might decrease the visibility of the trends presented in this chapter. However, I expected that these deviations would have averaged out.

Other electrolyte solutions According to the DFT calculations, on gibbsite the counter ion $\mathrm{Cl}^{-}$adsorbs on top of the adsorbed $\mathrm{Ca}^{2+}$-ions at high salt concentrations. To investigate the adsorption balance between the calcium and chloride ions, divalent ion solutions with different co- and counter-ions were investigated. In figure 6.6 the results for a $\mathrm{Ca}\left(\mathrm{NO}_{3}\right)_{2}, \mathrm{BaCl}_{2}$ and $\mathrm{CaBr}_{2}$ solution are shown.

Figure 6.6 shows the interaction force $\Pi_{T O T}-\Pi_{v d W}$ (where $R=10 \mathrm{~nm}$ ) on silica (solid curves) and gibbsite (dashed lines). The results are an average of 25 approaches and come from a single experiment for each condition. The $\mathrm{pH}$ of these solutions was not changed, and measured to be $\mathrm{pH} 5.2,5.7$ and 5.8 for $\mathrm{BaCl}_{2}, \mathrm{Ca}\left(\mathrm{NO}_{3}\right)_{2}$ and $\mathrm{CaBr}_{2}$ at $100 \mathrm{mM}$, respectively. The concentration at which the repulsive interaction force on silica is screened is for all three electrolyte solutions around $10 \mathrm{mM}$, which is similar to the result obtained for $\mathrm{CaCl}_{2}$ at $\mathrm{pH}$ 6. Furthermore, the overall trends found for the other ion type solutions are similar to those found for $\mathrm{CaCl}_{2}$, where the repulsive disjoining pressure on silica, and the attractive disjoining pressure on gibbsite decreases for increasing salt concentration. The Debye length for 


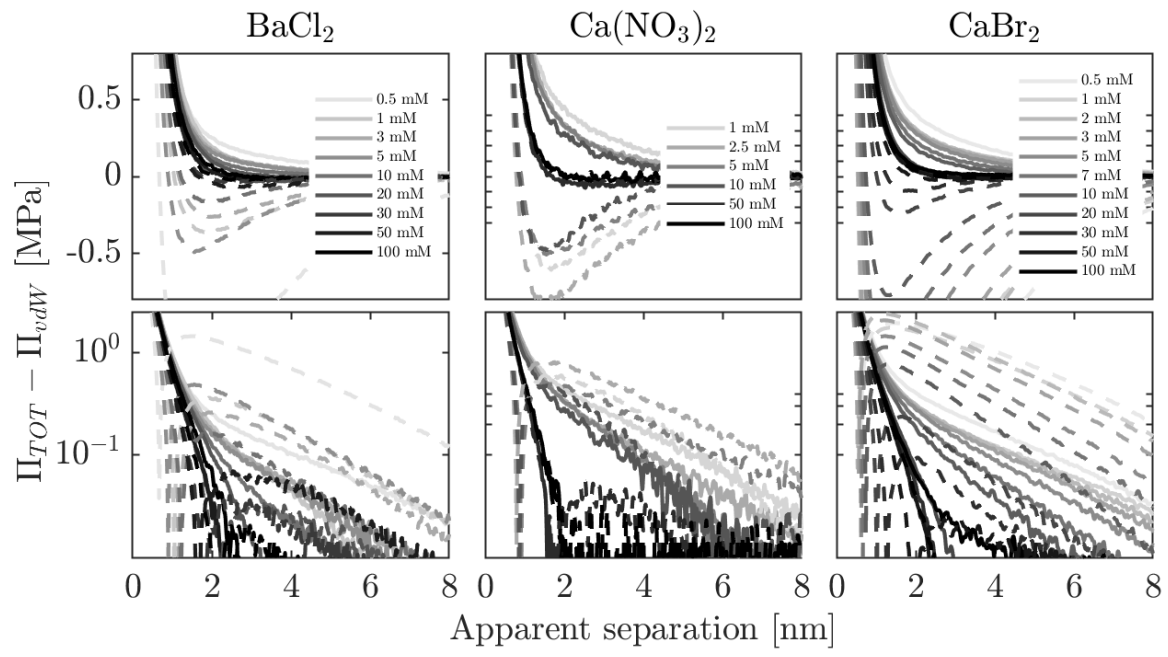

Figure 6.6: Disjoining pressure $\left(\Pi_{\text {total }}-\Pi_{v d W}\right)$ on gibbsite (dashed) and silica (solid) in various divalent ion electrolyte solutions. Increasing darkness is increasing ionic strength. The curves shown are an average of 25 approaches. The $\mathrm{pH}$ of these solutions were not adapted. Tip and $\mathrm{pH}$ (at $100 \mathrm{mM}$ ) parameters for $\mathrm{BaCl}_{2}, \mathrm{Ca}\left(\mathrm{NO}_{3}\right)_{2}$ and $\mathrm{CaBr}_{2}: \mathrm{pH}=$ $5.2,5.7$ and 5.8, $R_{\text {tip }}=25,15$ and $16 \mathrm{~nm} . \omega=45.2,50.7$ and $30.1 \mathrm{kHz} . k=2.51,3.14$ and $1.42 \mathrm{~N} \mathrm{~m}^{-1} . Q=3.36,4.2$ and 2.96 . 


\begin{tabular}{lccccccccc} 
Expected & 7.8 & 5.5 & 3.9 & 3.2 & 2.5 & 2.1 & 1.7 & 1.0 & 0.8 \\
$\mathcal{K}^{-1}(\mathrm{~nm})$ & $(0.5)$ & $(1)$ & $(2)$ & $(3)$ & $(5)$ & $(10)$ & $(20)$ & $(30)$ & $(50)$ \\
\hline $\mathrm{BaCl}_{2}$ & 3.0 & 1.5 & & 2.1 & 1.9 & 1.0 & 0.6 & 0.4 & 0.3 \\
$\mathrm{Ca}\left(\mathrm{NO}_{3}\right)_{2}$ & & 2.4 & 2.2 & & 1.4 & 1.5 & & & 0.3 \\
$\mathrm{CaBr}_{2}$ & 2.8 & 3.0 & 2.5 & 2.4 & 1.8 & 1.0 & 0.5 & 0.4 & 0.3
\end{tabular}

Table 6.2: Fitted decay lengths using $\kappa^{-1}=0.174 / \sqrt{I(M)}$ in $\mathrm{nm}$. The expected ionic concentration in between brackets. Data fitted from figure 6.6.

these measurements appear to be similar to that of calcium chloride, albeit slightly shorter (see table 6.2).

An interesting aspect of the results is that the minimum repulsive pressure for silica occurs at $50 \mathrm{mM}$, and increases for $100 \mathrm{mM}$ slightly. This is especially visible for $\mathrm{Ca}\left(\mathrm{NO}_{3}\right)_{2}$, but also occurs for $\mathrm{BaCl}_{2}$ and $\mathrm{CaBr}_{2}$. This slight increase is reflected in the diffuse layer charge calculations (figure 6.7) as well, where the charge value is higher at $100 \mathrm{mM}$ than at $50 \mathrm{mM}$.

Diffuse layer charge To obtain quantitative results from these measurements, we extract the effective surface charge using the procedure described in chapter 4.2.4. This will also give more insight into the results on gibbsite, since the measured disjoining pressures on gibbsite depend on the charge of the tip. The results for the diffuse layer charge calculations are shown in figure 6.7.

In figure 6.7 we can see the effective surface charge for silica (bottom) and gibbsite (top), for calcium chloride (left) and the other used electrolyte solutions (right). The error bars in the figure are based on the diffuse layer charge fitting results when the fit-range is changed $0.5 \mathrm{~nm}$ in both directions. The spread in the value determines the size of the errorbar. The error bars for the solutions above $10 \mathrm{mM}$ is substantial. This is a result of the fact that there is hardly any electrostatic force to fit to. What we can see from the diffuse layer charge on silica is that it does not depend significantly on the calcium chloride solution. Moreover, the non-monotonic behavior of the diffuse layer charge on gibbsite is observed in the work of Siretanu et al. [6] as well. This is especially true for the $\mathrm{BaCl}_{2}$ and $\mathrm{CaBr}_{2}$ solutions. Note that the peak is shifted to a higher concentration compared to the previous work, which could be caused by the fact that ionic concentrations might be lower than expected. The extracted values for 

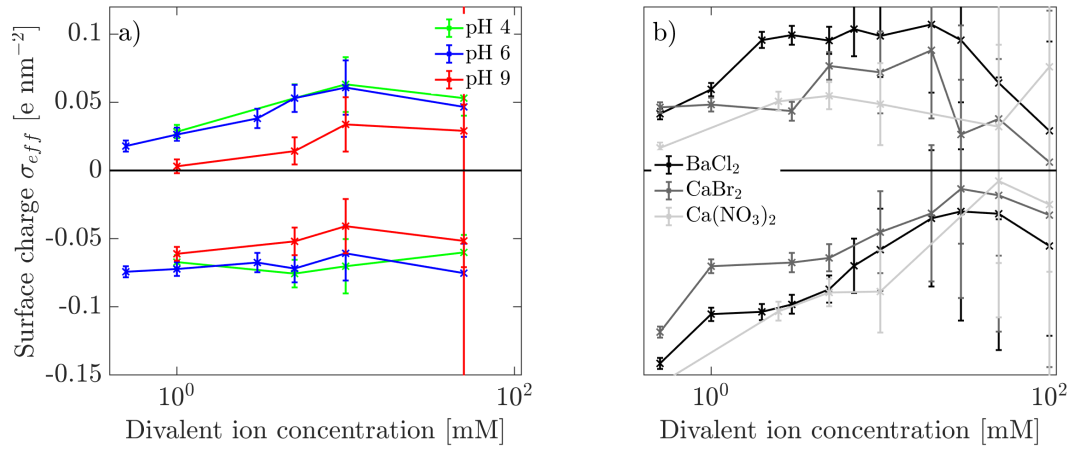

Figure 6.7: Calculated effective surface charge $\sigma_{\text {eff }}$ on silica (bottom) and gibbsite (top) as function of divalent ion concentration, measured in various $\mathrm{pH}$ and electrolyte solutions. In a) the diffuse layer charge shown on gibbsite and silica for different $\mathrm{pH}$ solutions as a function of $\mathrm{Ca}^{2+}$ concentration. In $\left.b\right) \sigma_{\text {eff }}$ is shown for three different electrolyte solutions $\left(\mathrm{Ca}\left(\mathrm{NO}_{3}\right)_{2}, \mathrm{CaBr} r_{2}\right.$ and $\left.\mathrm{BaCl}_{2}\right)$ as function of the divalent ion concentration. For these solutions the $\mathrm{pH}$ was not changed.

the diffuse layer charge are in the same order as reported by Siretanu et. al..

Somewhat surprising is the decreased effective surface charge over the complete calcium chloride concentration for $\mathrm{pH}_{9}$ compared to $\mathrm{pH}_{4}$ and 6 . This is different from what is observed in chapter 3 figure 3.5 and chapter 4 figure 4.10 but also in the literature $[10]$. Note, these results are for monovalent salts only. In chapter 3 we saw that the $\mathrm{pH}$ dependence is not necessarily described by a silanol deprotonation and a cation adsorption reaction only. We were unable to fit a $2 \mathrm{pK}$ model over a wide range of $\mathrm{pH}$ and $\mathrm{NaCl}$ concentration with a single $\mathrm{pK}$ pair. This suggests that there are additional surface reactions taking place at the interface. As already mentioned in the introduction here, there is evidence that $\mathrm{OH}^{-}$is involved in the adsorption of $\mathrm{Ca}^{2+}$ ions on the gibbsite surface. When the $\mathrm{pH}$ is increased, more $\mathrm{OH}^{-}$is available in the bulk, presumably aiding in the additional adsorption of $\mathrm{Ca}^{2+}$ resulting in a lower diffuse layer charge. These sort of surface reactions are usually not taken into account when modeling ion adsorption at the solid liquid interface. 


\subsection{Conclusion}

In this chapter, I characterized the diffuse layer charge on silica and the basal plane of gibbsite as a function of divalent ion concentration and $\mathrm{pH}$. I found that the effective surface charge on gibbsite increases up to a divalent ion concentration of $\approx 10 \mathrm{mM}$, and then decreases again. The measured effective surface charge is $\mathrm{pH}$ dependent on gibbsite and silica. For silica I found that for an increasing $\mathrm{pH}$ (from 6 to 9), the diffuse layer charge decreases, as is not expected from monovalent electrolyte studies. The origin could lie in the involvement of $\mathrm{OH}^{-}$in the adsorption of divalent ions on the gibbsite basal plane. These results suggest that hydration and water ions play an important role in the ion adsorption at the solid liquid interface and that their involvement has been underestimated in the past. 


\section{Bibliography}

[1] F. Mugele, B. Bera, A. Cavalli, I. Siretanu, A. Maestro, M. Duits, M. CohenStuart, D. van den Ende, I. Stocker, and I. Collins, "Ion adsorption-induced wetting transition in oil-water-mineral systems," Sci Rep, vol. 5, p. 10519, 2015.

[2] P. F. Mugele, I. Sîretanu, N. Kumar, B. Bera, L. Wang, M. Maestro, D. M. Duits, D. H. v. d. Ende, and I. Collins, "Charge control and wettability alteration at solid-liquid interfaces," 2014.

[3] F. Mugele, I. Siretanu, N. Kumar, B. Bera, L. Wang, R. de Ruiter, A. Maestro, M. Duits, D. van den Ende, and I. Collins, "Insights from ion adsorption and contact-angle alteration at mineral surfaces for low-salinity waterflooding," SPE Journal, 2016.

[4] C. A. J. Appelo and D. Postma, Geochemistry, groundwater and pollution. CRC press, 2004.

[5] G.V. Franks and Y. Gan, "Charging behavior at the alumina-water interface and implications for ceramic processing," Journal of the American Ceramic Society, vol. 90, no. 11, pp. 3373-3388, 2007.

[6] I. Siretanu, D. Ebeling, M. P. Andersson, S. L. Stipp, A. Philipse, M. C. Stuart, D. van den Ende, and F. Mugele, "Direct observation of ionic structure at solidliquid interfaces: a deep look into the stern layer," Sci Rep, vol. 4, p. 4956, 2014.

[7] A. M. Wierenga, T. A. J. Lenstra, and A. P. Philipse, "Aqueous dispersions of colloidal gibbsite platelets: synthesis, characterisation and intrinsic viscosity measurements," Colloids and Surfaces a-Physicochemical and Engineering Aspects, vol. 134, no. 3, pp. 359-371, 1998.

[8] J. N. Israelachvili and R. M. Pashley, "Molecular layering of water at surfaces and origin of repulsive hydration forces," Nature, vol. 306, no. 5940, pp. 249250,1983 .

[9] S. J. O'Shea and M. E. Welland, "Atomic force microscopy at solid-liquid interfaces," Langmuir, vol. 14, no. 15, pp. 4186-4197, 1998. 
[10] M. Kobayashi, F. Juillerat, P. Galletto, P. Bowen, and M. Borkovec, "Aggregation and charging of colloidal silica particles: Effect of particle size," Langmuir, vol. 21, no. 13, pp. 5761-5769, 2005. 


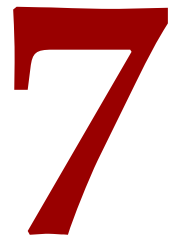

\section{Conclusions and outlook}

IN THIS THESIS, I investigated the interaction forces and surface charging behavior of different materials in different aqueous electrolyte solutions using high resolution AFM. In the introduction I established that in order to adequately understand the mechanisms underlying the low salinity water flooding (LSFW) effect a better understanding of the solid liquid interface is needed. The results presented in this thesis are applicable beyond the field of oil recovery and clay science and are more fundamental in nature. Below, I present the highlight of my findings.

\subsection{Conclusions}

In chapter 2 I gave a description of the solid liquid interface and expanded on the theoretical framework with the exponential surface complexation model (ESC model). With the use of the ESC model I was able to extract diffuse layer charges within $1 \%$ 
of a 2 pK surface complexation model. The use of the ESC model enables the separation of surface chemistry from diffuse layer charge measurements. This proved useful, because in chapter 3 I showed that even for a well studied system as the silicaelectrolyte interface many uncertainties remain about its surface chemistry. When using a $2 \mathrm{pK}$ model to determine the equilibrium constants for the deprotonation of the silanol groups and cation adsorption, their values turned out to be $\mathrm{pH}$ dependent. This means that a single $\mathrm{pK}$ pair is unable to describe the surface chemistry of silica over a wide range of salt concentrations and $\mathrm{pH}$. The determined diffuse layer charge values for silica were consistent between colloidal probes $(750 \mathrm{~nm})$ and sharp tips $(\approx 10 \mathrm{~nm})$. In chapter 4 I showed that for a different material (gibbsite) the results get increasingly more complex. Using high lateral resolution force spectroscopy (sharp tip) I revealed that the basal plane has a highly inhomogeneous and $\mathrm{pH}$ dependent charge density. The effective surface charge distribution on the basal plane correlated with the topographic defects, such as terrace steps on that plane. Also, the charge density at the defects showed to have a much stronger $\mathrm{pH}$ response than the basal plane. Describing such a surface using a single 2 pK surface complexation model with a single $\mathrm{pK}$ pair will be challenging. This is very different from how the gibbsite basal plane is generally modeled in experiments and simulations, leaving a large gap between theory and measurements. Chapter 5 shows that the solid liquid interface can get more complex, when hydration forces are taken into account. Here, I show unprecedented simultaneous probing of the hydration forces and DLVO forces on silica and a gibbsite nanoparticle. I show that the hydration structure and surface charge are not correlated and actually respond differently to various fluid compositions. The hydration forces are composed of an oscillatory force superimposed onto a monotonically decaying background force. The monotonic part of the hydration force is attractive on gibbsite and repulsive on silica for all measured fluid compositions. We cautiously theorize that this could be an effect of the different water molecule orientation in the layering at the gibbsite surface and silica surface. The number of oscillations and the strength of the oscillatory part of the hydration force is rather independent of the fluid composition. The oscillatory hydration force on the gibbsite basal plane is stronger than the silica surface, evidenced by the presence of more oscillations with a higher amplitude than silica. In chapter 6 I showed that when using divalent ions the force spectroscopy on silica and gibbsite can get more complex significantly. The interaction forces in divalent electrolyte solutions are small and the interaction range is short. Using large data sets to provide for a 
good statistical average and principal component analysis to improve the signal to noise ratio, improved the data marginally. However, we did find the non-monotonic adsorption behavior on gibbsite found in previous works, including that for other divalent ion electrolyte solutions. I also found that an increase of $\mathrm{pH} 6$ to 9 decreased the diffuse layer charge on silica for all $\mathrm{CaCl}_{2}$ concentrations, which is the opposite of what is found in chapter 3, 4 and in the literature for monovalent salts.

I demonstrated that AFM is a useful tool for surface charge characterization of the solid liquid interface with high lateral resolution. This technique provides unprecedented insight into the complex processes involved in the formation of the electric double layer on mineral surfaces. It can resolve the internal structure of the Stern layer, but it can also be used to measure hydration forces of diverse surfaces while simultaneously measuring electric double layer forces. Using these techniques, more materials can be studied in a more systematic fashion. I also demonstrated that our current understanding of the solid liquid interface is far from complete and that the involvement of hydration forces, water ions (like $\mathrm{OH}^{-}$) and surface defects have been underestimated in the past and are not properly taken into account in standard DLVO theory.

\subsection{Outlook}

\subsubsection{Diffuse layer charge vs intrinsic surface charge}

A question that arose in this thesis, is how is it that titration measurements can be described using relatively easy chemical speciation reactions, whereas with diffuse layer charge measurements it cannot. From chapter 3 we drew the conclusion that the diffuse layer charge as a function of sodium chloride concentration and $\mathrm{pH}$ cannot be described with a $2 \mathrm{pK}$ model. We have to note that a crucial difference between both types of charge is that titration measurements measure the intrinsic surface charge, whereas the diffuse layer charge contains excess count ions that screen the intrinsic surface charge and Stern layer. To get a better insight into why the modeling of both types of charge are so different, a systematic approach needs to be taken.

One way is to do titration measurements on several types of gibbsite and extract the relevant reaction constant. The same can be done using AFM, where the edges are 

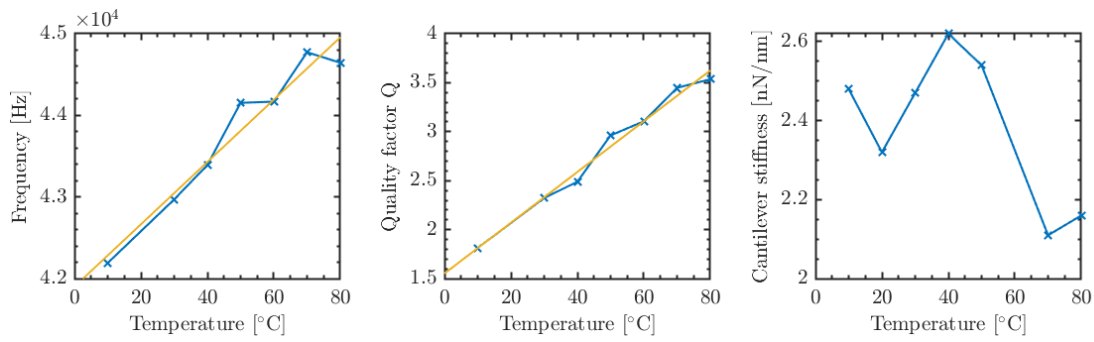

Figure 7.1: Experimental constants used in the qualitative analysis of the experiment that are temperature dependent, such as resonance frequency $\omega_{0}$, $Q$ and the spring constant. $\left.a\right)$ The resonance frequency shows to be linear in the 10 to $80{ }^{\circ} \mathrm{C}$ regime, with the approximation: $\omega_{\mathrm{o}}=38.2 T+41900$. With $T$ in Celsius. b) The quality factor can be approximated for this specific cantilever with $Q=0.026 T+1.55$. c) The cantilever stiffness, or spring constant does not seem to be linear with temperature. The routine used for determining the spring constant has a spread of around $0.1 \mathrm{~N} \mathrm{~m}^{-1}$. Experimental parameters at $30^{\circ} \mathrm{C}$ : $R_{\text {tip }} \approx 20 \mathrm{~nm}, \omega=43 \mathrm{kHz}, Q=2.4$ and $k=2.5 \mathrm{~N} \mathrm{~m}^{-1}$.

measured with one of the solutions mentioned in the previous section. Using high resolution atomic force microscopy, even the Stern layer can be studied.

\subsubsection{Reservoir conditions}

In this thesis the experiments have been performed under ambient conditions. This means at a temperature of $29^{\circ} \mathrm{C}$ and an ambient pressure of 1 bar. This is significantly different from reservoir conditions, where the pressure can rise up to a 100 bar and the temperature up to $200{ }^{\circ} \mathrm{C}$. In previous studies it has been found that both the temperature and pressure have a significant influence on the zeta potential of reservoir minerals. It is reported that the zeta potential grows more negative with increasing temperature, where each crystal has a characteristic rate ${ }^{[1,1,2]}$. Pressure has a $\mathrm{pH}$ and mineral type dependent influence, where for certain systems, such as quartz, the zeta potential increases and for example kaolinite, the zeta potential decreases. 


\section{Temperature}

The literature on temperature dependent measurements on clay minerals is limited. There is some literature on clays like goethite, rutile and kaolinite [3]-7], sandstone samples $[8,1,9]$ and aqueous dispersions $[10,11]$. Some interesting phenomenon can be observed, for example, the contact angle of a drop of aqueous solution in a oil system can change over $70^{\circ}$, when the temperature is changed from 40 to $60^{\circ} \mathrm{C}$ [12] . All these experiments are large scale experiments, providing a good statistical average. However, when trying to understand microscopic aspects of the charging mechanisms involved, this is a disadvantage. Despite that atomic force microscopy can provide a way to understand these microscopic aspects, literature on this subject is very limited. This has most likely to do with the technical complications that occur when AFM measurements are performed at variable temperatures. In the example of amplitude modulation force microscopy, a significant amount of experimental constants used in the qualitative part of the analysis of the experiment are only constants for a constant temperature. This means that many of these constants have to be redetermined at each temperature the experiment is performed. For example, changing the temperature changes the frequency response, resonance frequency and the spring constant of the cantilever, as shown in figure 7.1. Furthermore, the amplitude sensitivity also changes, albeit slightly, due to the changing refractive index of water [13]. The dissociation constant of water strongly depends on temperature as well, where a difference in $70^{\circ} \mathrm{C}$ changes the dissociation constant over 2 units. This will have a significant influence on the $\mathrm{pH}$ of the solution that is used. Moreover, the density of the water changes about $2.5 \%$ over a range of $70^{\circ} \mathrm{C}$, which influences the resonance frequency of the cantilever [14]. Other technical difficulties that have been experienced are the strong evaporation of the liquid, resulting in an ever-changing salt concentration and $\mathrm{pH}$, condensation on optics, resulting in loss of signal and thermal drift, which can be over $20 \mathrm{~nm} \mathrm{~s}$. The latter makes studying nanoparticles a challenge in particular. The increased temperature also enables contaminants to go to the tip more easily, because of the increased energy in the system. The risk of contaminating your tip at elevated temperatures is significant. This will have more effect on smaller cantilever tips than large tips, such as colloidal probes.

Preliminary results of the interaction stiffness as function of temperature for a silicasilica system is shown in figure 7.2 for sharp tips, and in figure 7.3 for a colloidal 


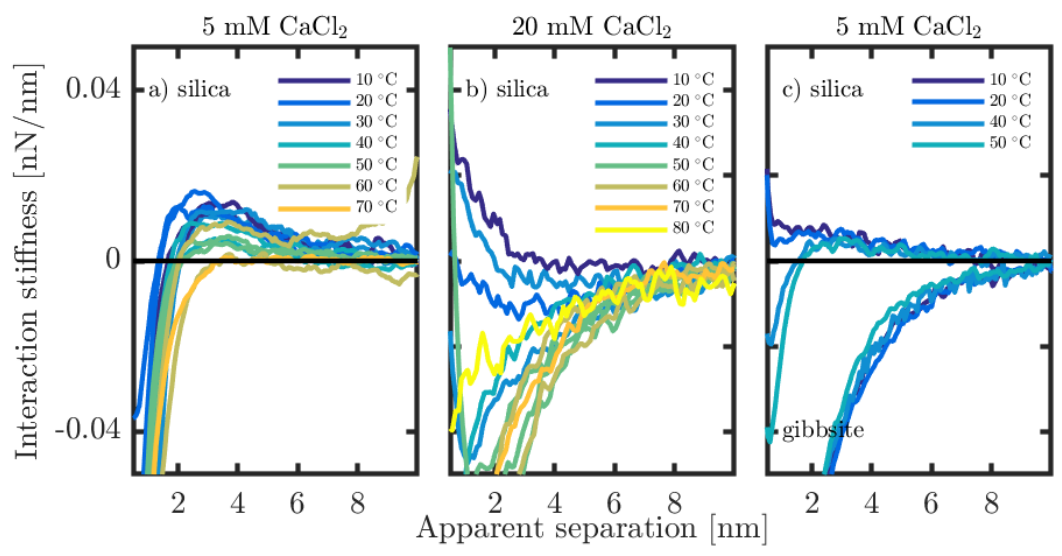

Figure 7.2: Interaction stiffness as function of temperature for three individual experiments. Measurements are an average of 100 approaches on silica in a) $5 \mathrm{mM} \mathrm{CaCl}_{2}$ solution. a) Each temperature was measured twice, consecutively, to measure the stability of the experiment. b) Data measured in a $20 \mathrm{mM} \mathrm{CaCl}$ solution. c) Measurement including approaches on gibbsite (lower graphs). Experimental parameters at $30^{\circ} \mathrm{C}$ for $a, b$ and $c$ : $Q=2.4,2.7$ and $3.4, \omega=40.9,45.1$ and $41.9 \mathrm{kHz}$ and $k=2.0,2.5$ and $2.0 \mathrm{~N} \mathrm{~m}^{-1}$. 


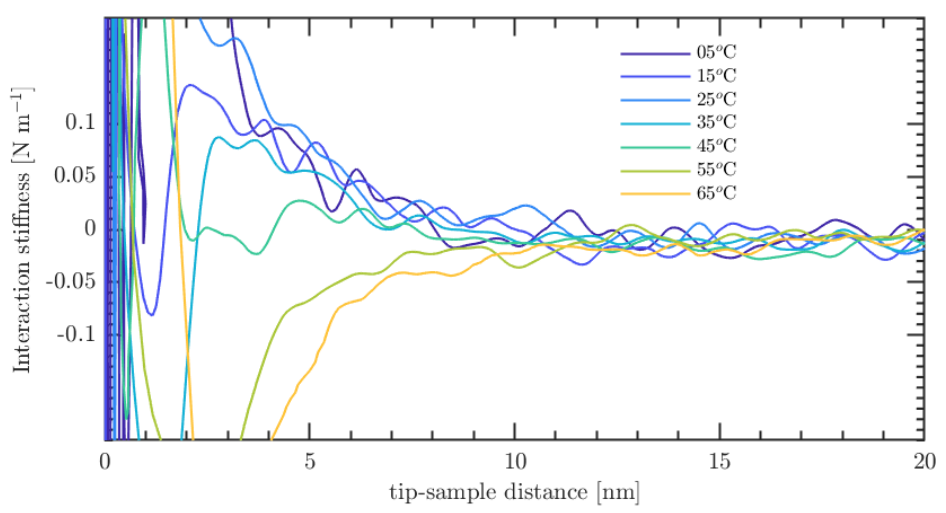

Figure 7.3: Interaction stiffness as function of temperature measured with a colloidal probe in $5 \mathrm{mM} \mathrm{CaCl}$ solution. Experimental parameters at $30^{\circ} \mathrm{C}: R_{t i p}=750 \mathrm{~nm}, Q=2.7$, $\omega=17.6 \mathrm{kHz}$ and $k=0.7 \mathrm{~N} \mathrm{~m}^{-1}$.

probe. All measurements show a decrease in repulsive interaction mainly. As mentioned before, contamination has a large influence on the reliability and reproducibility of these experiments. Using a large tip radius, as in figure $7 \cdot 3$, can mitigate the influence of tip contamination. In these experiments it was also observed that sample history played in important role. Repeating the experiment on the same sample, meaning a temperature sweep from 10 to $80^{\circ} \mathrm{C}$ can result in very different observed behavior. To conclude, reversibility and equilibration for temperature dependent measurements is an especially difficult challenge to overcome in order to produce reliable results.

\subsubsection{Hydration forces}

The monotonic contribution to the hydration force remains an open topic [15-21]. However, the results in this thesis perhaps show a hint of the origin of the force. It is important to realize that the monotonic contribution might not only be the result of the hydration force, but other short-range interactions as well or that is an artifact from the subtraction of the van der Waals force. The monotonic force is sensitive to this subtraction, especially for the symmetric case (silica-silica). The sign of the 
monotonic force in the asymmetric case (gibbsite-silica) did not depend on the subtraction of the van der Waals force within experimental error. To rule out the uncertainty for the symmetric case, more systematic experiments should be performed like the ones in chapter 5 . By using different systems (symmetric vs asymmetric and different electrolyte solutions) and taking into account the correct tip shape, the switching of the monotonic interaction force could be proven. This would improve the understanding on a topic that has been under debate for a long time. 


\section{Bibliography}

[1] K. Rodriguez and M. Araujo, "Temperature and pressure effects on zeta potential values of reservoir minerals," Journal of Colloid and Interface Science, vol. 300, no. 2, pp. 788-794, 2006.

[2] R. Ramachandran and P. Somasundaran, "Effect of temperature on the interfacial properties of silicates," Colloids and Surfaces, vol. 21, pp. 355-369, 1986.

[3] M. J. Angove, B. B. Johnson, and J. D. Wells, "The influence of temperature on the adsorption of cadmium(ii) and cobalt(ii) on kaolinite," Journal of Colloid and Interface Science, vol. 204, no. 1, pp. 93-103, 1998.

[4] L. G. J. Fokkink, A. Dekeizer, and J. Lyklema, “Temperature-dependence of the electrical double-layer on oxides - rutile and hematite," Journal of Colloid and Interface Science, vol. 127, no. 1, pp. 116-131, 1989.

[5] B. B. Johnson, "Effect of $\mathrm{ph}$, temperature, and concentration on the adsorption of cadmium on goethite," Environmental Science and Technology, vol. 24, no. 1, pp. $112-118,1990$.

[6] S. Subramanian, J.A. Schwarz, and Z. Hejase, "The temperature-dependence of the point of zero charge of gamma-al203, tio2, and physical mixtures," Journal of Catalysis, vol. 117, no. 2, pp. 512-518, 1989.

[7] P. H. Tewari and A. W. McLean, "Temperature dependence of point of zero charge of alumina and magnetite," Journal of Colloid and Interface Science, vol. 40, no. 2, pp. 267-272, 1972.

[8] P. M. Reppert and F. D. Morgan, “Temperature-dependent streaming potentials: 2. laboratory," Journal of Geophysical Research-Solid Earth, vol. 108, no. B1 $1,2003$.

[9] J. Vinogradov and M. D. Jackson, "Zeta potential in intact natural sandstones at elevated temperatures," Geophysical Research Letters, vol. 42, no. 15, pp. 62876294, 2015 .

[10] M. F. Mohtadi and P. N. Rao, "Effect of temperature on flocculation of aqueous dispersions," Water Research, vol. 7, no. 5, pp. 747-767, 1973. 
[11] A. L. Valdivieso, J. L. R. Bahena, S. Song, and R. H. Urbina, “Temperature effect on the zeta potential and fluoride adsorption at the alpha-al203/aqueous solution interface," Journal of Colloid and Interface Science, vol. 298, no. 1, pp. 1-5, 2006.

[12] M. E. J. Haagh, N. Schilderink, M. H. G. Duits, I. Siretanu, F. Mugele, and I. R. Collins, "Salinity-dependent contact angle alteration in oil/brine/silicate systems: The effect of temperature," Journal of Petroleum Science and Engineering, vol. 165, pp. 1040-1048, 2018.

[13] A. Bashkatov and E. Genina, Water refractive index in dependence on temperature and wavelength: A simple approximation, vol. 5068. 2003.

[14] F. Tabbutt, "Water: A matrix of life, 2nd edition (franks, felix)," Journal of Chemical Education, vol. 78, no. 5, p. 593, 2001.

[15] J. N. Israelachvili, "Measurement of forces between surfaces immersed in electrolyte-solutions," Faraday Discussions, vol. 65, pp. 20-24, 1978.

[16] J. P. Chapel, "Electrolyte species dependent hydration forces between silica surfaces," Langmuir, vol. 10, no. 11, pp. 4237-4243, 1994.

[17] A. C. Cowley, N. L. Fuller, R. P. Rand, and V. A. Parsegian, "Measurement of repulsive forces between charged phospholipid bilayers," Biochemistry, vol. 17, no. 15 , pp. $3163-3168,1978$.

[18] D. M. Leneveu, R. P. Rand, and V. A. Parsegian, "Measurement of forces between lecithin bilayers," Nature, vol. 259, no. 5544, pp. 601-603, 1976.

[19] R. M. Pashley, "Hydration forces between mica surfaces in aqueous electrolyte solutions," Journal of Colloid and Interface Science, vol. 8o, no. 1, pp. 153-162, 1981.

[20] V. A. Parsegian and T. Zemb, "Hydration forces: Observations, explanations, expectations, questions," Current Opinion in Colloid \& Interface Science, vol. 16, no. 6, pp. $618-624,2011$.

[21] B. R. Shrestha and X. Banquy, "Hydration forces at solid and fluid biointerfaces,” Biointerphases, vol. 11, no. 1, p. $018907,2016$. 


\section{Summary}

For enhanced oil recovery at sea a technique called low salinity water flooding can be used. Instead of the standard procedure of injecting seawater into the reservoir directly, the salinity of the sea water is reduced before injection. This increases the amount of recovered oil for some sandstone reservoirs, but not for all. The reason for the improved recovery rate is generally believed to be an overall increase of the water affinity of the sandstone reservoir, resulting in more oil to be displaced. However, as mentioned, this does not hold for all sandstone reservoirs.

The question is why.

To answer this, we need a better understanding of the underlying mechanisms of the wettability alteration (change in affinity) of the oil reservoir. Due to the high degree of complexity, those mechanisms are still poorly understood. Not only because it is multi-scale problem (a reservoir can be kilometers long, while the chemical reactions play at the micro-scale), a multi-phase problem (a reservoir contains oil, water and rock), but also because of the high variety in organic compounds (like aromatic hydrocarbons) and salts $\left(\mathrm{NaCl}, \mathrm{KCl}, \mathrm{MgCl}_{2}\right.$ and $\left.\mathrm{CaCl}_{2}\right)$ present in the reservoir.

In order to improve the understanding of the fundamental forces at play, it is essential to represent the oil reservoir by simple, yet adequate model systems that capture all the relevant phenomena. From these models we can study the interaction forces that govern the low salinity effect. These are forces such as electrostatic forces, van der Waals forces and hydration forces. They determine largely how the different surfaces in the reservoir interact with their environment. In this work we study several model systems, by measuring and modeling the effects of external stimuli, such as salt concentration, $\mathrm{pH}$ and ion type on the interaction force using Atomic Force Microscopy 
(AFM). The AFM allows us to measure interaction forces down to the nano-Newton range. When sharp tips are used forces with high lateral and longitudinal resolution can be obtained.

Measuring these different types of interaction forces with AFM is not only relevant in the context of oil recovery. They find their relevance in the biologica, (geo) chemical and technological context as well. However, in this thesis we use the context of oil recovery to study the different types of interaction forces.

In Chapter 3 we look at one of the most occurring materials in a sandstone reservoir, silica. We study the ionic strength and $\mathrm{pH}$ dependence of the diffuse layer charge of the silica-electrolyte interface. We use an exponential surface charge (ESC) - potential relation to extract diffuse layer charge values from measurements using an AFM with colloidal probes. This way no assumptions need to be made about the chemical composition of the surface. We analyze and compare the results with a 2-pK model to verify the findings of the ESC model. We find that the 2-pK model can accurately describe the diffuse layer charge, albeit at a single $\mathrm{pH}$ value. The values obtained using sharp tips and colloidal probes are all consistent.

The modeling of a relatively simple model surface can already be a challenge. It is a continuous struggle between model complexity and model accuracy. With the increase of computational power, the models have become increasingly complex and in most cases more accurate too. In Chapter 4 we look at the modeling of clay minerals. We do this by measuring the diffuse layer charge of gibbsite as a function of sodium salt concentration and $\mathrm{pH}$. Gibbsite is a clay mineral abundantly available in sandstone oil reservoirs. Due to its high surface to volume ratio and high reactivity it may have a significant influence in the low salinity effect. We quantify the diffuse layer charge over 150 by $150 \mathrm{~nm}$ with a $2.5 \mathrm{~nm}$ resolution on a single particle. We analyze our results and compare the outcome with results from commonly used models and experiments. We find a diffuse layer charge heterogeneity that correlates with the presence of defects on the basal plane of the gibbsite basal plane. The smooth regions on gibbsite have a small positive charge and do not show much $\mathrm{pH}$ dependence. The defect sites, however, show a relatively large change in charge density between $\mathrm{pH} 6$ and 9. Taking into account the size of the tip, we conclude that the at $\mathrm{pH} 9$ the defect sites are negatively charged. This is something that is hardly taken into account when modeling clay mineral surfaces. 
The diffuse layer charge determined in previous chapters governs a great deal on how these surfaces interact with their environment. The forces taken into account in these previous chapters are the van der Waals force and the electric double layer force. However, another significant interaction force is the hydration force. In Chapter 5 we study the hydration force as a function of sodium salt concentration and $\mathrm{pH}$ on silica and gibbsite. By measuring the diffuse layer charge and the hydration force simultaneously, we can generate a more complete picture of the forces at play at the solid-liquid interface of clay and rock surfaces. We model the full interaction force and analyze the various system parameters as a function of salt concentration and $\mathrm{pH}$. We find that the monotonic part of the hydration force is salt and $\mathrm{pH}$ dependent, whereas the structural part does not significantly change over $\mathrm{pH}$ or salt concentration

In previous chapters we studied monovalent salts only, mainly because the modeling is more straightforward and the forces are easier to measure. In the low salinity effect however, the divalent ion content of the injection water is found to be a key component. In chapter 6 we aim to measure the diffuse layer charge dependence of gibbsite for divalent salts. The absolute forces are small and the range is short. We analyze the interaction forces, calculate the diffuse layer charge and analyze the observed trends as a function of salt concentration, salt type and $\mathrm{pH}$. We find that the observed trends are as expected (for increasing salt concentration the relative diffuse large charge increases and the decay length shortens). However, the decay lengths of the interaction forces are not as expected.

In the last chapter several questions that arose during this work are discussed. One of those is the effect of temperature on the diffuse layer charge. The experiments in this thesis are performed at room temperature, whereas the oil reservoirs are usually at a much higher temperature. Also the challenges and uncertainties of the measurement techniques used in this work are reflected upon. 


\section{Samenvatting}

Voor de verbeterde oliewinning op zee wordt een techniek gebruikt die lage saliniteit waterinjectie genoemd wordt. In plaats van de standaard procedure, waar zeewater direct in het oliereservoir wordt geïjecteerd, wordt het zoutgehalte van het zeewater gereduceerd voordat het geïnjecteerd wordt. Dit verhoogt voor sommige zandsteenoliereservoirs de hoeveelheid olie de gewonnen kan worden. De reden voor de verbeterde oliewinning wordt over het algemeen toegeschreven aan een verbetering van de wateraffiniteit van het zandsteenreservoir, waardoor meer olie verplaatst kan worden. Echter, geldt dit niet voor alle zandsteenoliereservoirs.

De vraag is waarom.

Om dit te kunnen beantwoorden, hebben we een beter begrip nodig van de onderliggende mechanismen van de bevochtingsverandering (verandering van de affiniteit) van het reservoir. Door de hoge mate van complexiteit, zijn die mechanismen nog niet goed begrepen. Dit komt niet alleen doordat het probleem op meerdere lengteschalen speelt (een reservoir kan kilometers lang zijn, terwijl chemische reacties zich afspelen op de microschaal), er meerdere fasen (olie, water en rots) met elkaar in contact zijn, maar ook vanwege de hoge variëteit in organische moleculen (zoals aromatische koolwaterstoffen) en zouten $\left(\mathrm{NaCl}, \mathrm{KCl}, \mathrm{MgCl}_{2}\right.$ and $\left.\mathrm{CaCl}_{2}\right)$.

Het verbeteren van het begrip van de fundamentele krachten die plaatsvinden, kan gerealiseerd worden door het oliereservoir te modelleren met simpele en passende modelsystemen die alle relevante verschijnselen bevatten. Vanuit deze modellen kunnen de interactiekrachten die verantwoordelijk zijn voor het lage saliniteit waterinjectie effect bestudeerd worden. Dit zijn krachten zoals de elektrostatische kracht, Van der Waals kracht en de hydratatiekracht. Deze krachten bepalen grotendeels hoe de verschillende oppervlakken in het reservoir op hun omgeving reageren. Echter, 
deze interactiekrachten vinden hun relevantie niet alleen in de context van oliewinning. Het bestuderen van deze krachten is ook relevant in de context van biologische processen, in de (geo)chemie en technologische processen. We zullen in deze thesis echter werken vanuit de context van oliewinning.

In deze thesis bestuderen we verschillende modelsystemen door metingen te doen en simulaties te maken van de effecten van externe stimuli, zoals zoutconcentratie, $\mathrm{pH}$ en ionsoort op de interactie kracht. Dit doen we met een fundamentele kracht microscoop (AFM). Met een AFM kunnen krachten tot op een nano Newton gemeten worden. Wanneer we een scherpe naald gebruiken kan dit gedaan worden met een hoge laterale en longitudinale resolutie.

In hoofdstuk 3 kijken we naar één van de meest voorkomende materialen in een zandsteenreservoir, namelijk silica. We bestuderen de afhankelijkheid van de oppervlaktelading van het silica-elektrolyt grensvlak op het zoutgehalte en de $\mathrm{pH}$ van de oplossing. We gebruiken een exponentiële oppervlaktelading (ESC) - potentiaal verhouding om de oppervlaktelading waardes uit de metingen met een colloïdale naald te halen. Met deze techniek hoeven er geen aannames gemaakt te worden over de chemische samenstelling van het oppervlak. We analyseren en vergelijken de resultaten met een 2 -pK model om onze resultaten uit het ESC model te verifiëren. Hieruit blijkt dat het 2-pK model de oppervlaktelading goed kan beschrijven, echter voor een enkele $\mathrm{pH}$ waarde. De oppervlakteladingwaardes verkregen met een scherpe naald en een colloïdale naald komen overeen.

Het modelleren van relatief simpele modeloppervlak kan al een uitdaging zijn. Het is een voortdurende worsteling tussen modelcomplexiteit en modelnauwkeurigheid. Met de verbetering van de processorsnelheid kunnen de modellen complexer worden, waardoor in de meeste gevallen de modellen ook realistischer zijn geworden.In hoofdstuk 4 bestuderen we het modelleren van kleimineralen. Dit doen we door de oppervlaktelading van gibbsite te meten als een functie van natriumzoutgehalte en $\mathrm{pH}$. Gibbsite is een kleimineraal dat veelvuldig aanwezig is in zandsteenoliereservoirs. Door zijn hoge oppervlak-volume verhouding zou het een significante aandeel kunnen hebben in het lage saliniteit effect. We kwantificeren de oppervlaktelading over een oppervlak van 150 bij $150 \mathrm{~nm}$ met een $2.5 \mathrm{~nm}$ resolutie op een enkel gibbsite deeltje. We analyseren onze resultaten en vergelijken die met resultaten van gebruikelijke modellen en experimenten. We zien dat de oppervlakteladingheterogen- 
iteit corroleert met de aanwezigheid van oppervlaktedefecten op het hoofdoppervlak van gibbsite. De gladde gebieden op het hoofdoppervlak hebben een kleine positieve lading en zijn nauwelijks afhankelijk van $\mathrm{pH}$. De gebieden met oppervlaktedefecten daarentegen, vertonen een relatief groot verschil in oppervlakteladingsdichtheid tussen $\mathrm{pH} 6$ en 9. Als er rekening gehouden wordt met de grootte van de naald, concluderen we dat de oppervlaktedefecten een negatieve oppervlaktelading hebben bij pH 9. Dit is iets waar nauwelijks rekening mee gehouden wordt in modellen van kleimineraaloppervlakken.

De oppervlaktelading bepaald grotendeels hoe het oppervlak interacteert met zijn omgeving. De krachten die gemeten zijn in de vorige hoofdstukken zijn de Van der Waals kracht en de elektrostatische kracht. Echter, een andere belangrijke interactiekracht is de hydratatiekracht. In hoofdstuk 5 bestuderen we de hydratatie kracht als functie van natriumzoutconcentratie en $\mathrm{pH}$ op silica en gibbsite. Door het meten van de oppervlaktelading en hydratiekracht tegelijkertijd, verkrijgen we een completer beeld van de krachten die spelen op het oppervlak-elektrolytgrensvlak van klei en rotsoppervlakken. We modelleren de volledige interactie kracht en analyseren verscheidene systeemparameters als functie van zoutconcentratie en $\mathrm{pH}$. Hieruit blijkt dat het monotonische gedeelte van de hydratatiekracht afhankelijk is van de $\mathrm{pH}$ en zoutconcentratie. Daarentegen laat het structurele gedeelte van de hydratatiekracht weinig afhankelijkheid zien.

In voorgaande hoofdstukken bestudeerden we alleen monovalente zouten, voornamelijk omdat het modelleren eenvoudiger en directer is en de krachten eenvoudiger te meten zijn. In het lage saliniteit effect blijkt dat de divalente ionen in het injectiewater een sleutelrol hebben. In hoofdstuk 6 is het doel om de oppervlakteladingafhankelijkheid van divalente ionen te meten van gibbsite en silica. De absolute krachten zijn kleiner en ook de interactielengtes zijn korter vergeleken met monovalente ionen. We analyseren de interactiekrachten, berekenen de oppervlakteladingsdichtheid en analyseren de trends als functie van zoutconcentratie, ionsoort en $\mathrm{pH}$. We zien dat de gevonden trends zoals verwacht zijn (een hogere zoutconcentratie zorgt voor een grotere oppervlaktelading maar een kortere vervallengte). De vervallengte is langer dan verwacht.

In het laatste hoofdstuk worden verscheidene vragen behandeld die tijdens het maken van het thesis naar voren kwamen. Eén van die vragen is het effect van de temper- 
atuur op de oppervlaktelading. De experimenten uitgevoerd in dit werk zijn gedaan op kamertemperatuur, echter zijn de temperaturen in het oliereservoir meestal veel hoger. Ook worden de uitdagingen en onzekerheden van de gebruikte meettechnieken besproken. 


\section{List of publications}

\section{First author}

1. A. Klaassen, F. Liu, D. van den Ende, F. Mugele, and I. Siretanu, "Impact of surface defects on the surface charge of gibbsite nanoparticles," Nanoscale, 2017

\section{To be published}

1. A. Klaassen, D. Van den Ende, F. Mugele, and I. Siretanu, "On the relation between colloidal electrostatic and hydration forces on mineral surfaces in ambient electrolytes of variable composition: An atomic force microscopy study," 2021

2. A. Klaassen, D. Van den Ende, I. Siretanu, and F. Mugele, "Ionic strength and ph dependence of the surface charge of silica-electrolyte interfaces, measured with afm," 2021

\section{Others}

1. F. Liu, A. Klaassen, C. Zhao, F. Mugele, and D. Van den Ende, "Electroviscous dissipation in aqueous electrolyte films with overlapping electric double layers," The Journal of Physical Chemistry B, vol. 122, 102017

2. N. Kumar, C. Zhao, A. Klaassen, D. van den Ende, F. Mugele, and I. Siretanu, "Characterization of the surface charge distribution on kaolinite particles using high resolution atomic force microscopy," Geochimica et Cosmochimica Acta, vol. 175, pp. 100-1 12, 2016 
3. D. Ziegler, A. Klaassen, D. Bahri, D. Chmielewski, A. Nievergelt, F. Mugele, J.E. Sader, and P. D. Ashby, "Encased cantilevers for low-noise force and mass sensing in liquids," in 2014 IEEE 27 th International Conference on Micro Electro Mechanical Systems (MEMS), pp. 128-131, 2014

4. K. Wenderich, A. Klaassen, I. Siretanu, F. Mugele, and G. Mul, "Sorption-determined deposition of platinum on well-defined platelike wo3," Angewandte ChemieInternational Edition, vol. 53, no. 46, pp. 12476-12479, 2014 
"It is nice to be important, but it is more important to be nice."

Scooter - A German poet

\section{Acknowledgments}

I really enjoyed my $\mathrm{PhD}$. It was truly a beautiful period, with some stressful moments here and there, but let's not dwell on those. One of the better things is traveling for conferences to exotic places, like Veldhoven, Copenhagen and Bad Boekelo. But seriously, Cassis, Les Houches and San Francisco were real highlights of my PhD. Enjoying different cultures, exploring new places and learning about new science was a privilege to have experienced. Another thing is the freedom, which can actually be a pitfall too. I chose to spend (a bit of) my freedom on playing futsal, and it will be something I will miss dearly in the future. It was something I looked forward to, every week. The chance to release some stress, to clear your mind and meet other people from the university was a lot of fun. But of course, these and other great times were made possible by a lot of people.

First of all, I would like to thank Frieder for having me for such a long time. It all started with my bachelors assignment in Frieder's group a long time ago. Then I got my internship in the awesome Lawrence Berkeley National Laboratory through Frieder's efforts. I then carried out my masters thesis in Frieder's group and finally Frieder offered me a $\mathrm{PhD}$ position as well. It was a great time and I would like to thank you for the guidance through this period.

Dirk, I learned a lot while working with you as my supervisor. I really enjoyed your view on physics and your everlasting hunger to understand things. When I presented data, you never responded with "This data is wrong", but always with "That is interesting, how is this possible". That is a nice way to approach problems.

Thank you Igor, for all the help during my $\mathrm{PhD}$. You were always ready to listen to my problems and you were always full of ideas. Without your help, this thesis would look very different. But besides the science, I enjoyed the social time too. I loved being 
your personal driver on our trips to Brussels, Dortmund and other places.

Michel, it was a lot of fun teaching first years Dynamica with you. I learned a lot, and also a lot about helping fresh students through their very first university course. But I also enjoyed our chats about football and our friendly feud between PSV and Ajax. I did not enjoy some of our visits to the Twente-Ajax football matches that much though...

I also like to thank my thesis committee members for taking their time to critically read my thesis and provide feedback.

I would like to officially thank all my (former) colleagues from PCF, for providing me with a pleasant work-environment. Thanks to all my former group members: Sissi, Armando, Andrea, Duc, Rudy, Kartikya, Mariska, Dieter, Rielle, Jolet, Fei, Lei, Naveen, Davood, Aditya, Stelian, Nicolao, Ivo, Omkar, Somnath, Yan, Arjen, Edoardo, Olena, Chamy, Cunlu, Jorick, Zhantao, Edith, Lisette, Daniel H., Bijoy, 1 , and the group members when I left: Saravanna, Ashit, Carla, Duy, Beybin, Hao, Isabel, Daniel, Sachin, Ranabir, Simone, Michel, Cherry ;), Jun, Martin, Harmen and Nathalie. But a special thanks to the people (they know who they are) that made that $\mathrm{PhD}$ time just a 'little' bit more enjoyable, by listening to each other complain about work, making 'your mom' jokes, drinking beers, watching Star Wars, going to Happy Italy, having 'borrels', celebrate birthdays, playing boardgames, have barbecues and many other things. Thank you.

I like to say thanks to the futsal teams, PCF Strikers, POF United, Micronit and EMS. Our futsal matches kept me sane throughout the years. Daniel, Bijoy, Armando, Andrea, Maurice vD., Jorrit, Harmen, Roger, Ruben V., Mathijs vG., Bastiaan, Maurice W., Mathijs B., JJ, Anne, Ruben L., Cris, Thomas, Nando, Mark and Izak.

I also need to thank the people from Brunel, Julia, Bas, Bram, Laura, Roderick, Danny, Mark, Kevin, Marieke en Carla. They had to tolerate my presence for a whole month, which might have felt like an eternity.

Luuk, thanks for being such an awesome friend.

Friends in the backcorner, thank you for tolerating me as a friend and being the inspiration for my front cover.

\footnotetext{
${ }^{1}$ Fill in your name if $\mathrm{I}$ forgot to mention you
} 
I would like to thank my parents and my sister for their immense and unconditional care, love and support. But also for asking the right amount of times 'How is your thesis going?' (which was luckily not too often) and having faith in me finishing it.

And as last, most importantly, thank you Denise. I am glad that all the times that I talked about working in the weekend, I practically never did, but spend that time with you. Best way to spend time. Ever. 


\section{About the author}

Aram was born on the 9th of February, 1988, in Silvolde (Wisch), the Netherlands. After completing secondary education at the Almende College locatie Isala in Silvolde in 2006, he studied Applied Physics at the university of Twente. He received his BSc. degree in 2011 and in 2014 Aram obtained his Master's degree in Fluid Physics. As part of the study program, Aram spend three months in the Lawrence Berkeley National Laboratory (USA) working on the dynamics

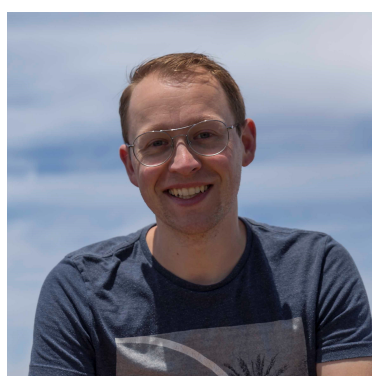
of encased cantilevers. In 2014, he started his $\mathrm{PhD}$ research in the Physics of Complex Fluids group within the MESA+ Institute for Nanotechnology and the Department of Science and Technology of the university of Twente, under supervision of prof. Frieder Mugele and dr. Igor Siretanu. The results of this reserach are presented in this thesis. 